\title{
Femto Joule per Conversion 8-Bit Comparator Assisted Binary Search Analog to Digital Converter
}

by

Octavian Stelescu, B.Eng.

\begin{abstract}
A thesis submitted to the
Faculty of Graduate Studies and Research

in partial fulfillment of the requirements for the degree of
\end{abstract}

Master of Applied Science in Electrical Engineering

Ottawa-Carleton Institute for Electrical and Computer Engineering

Department of Electronics

Carleton University

Ottawa, Ontario

April, 2012

(C) Copyright

Octavian Stelescu, 2012 
Library and Archives

Canada

Published Heritage

Branch

395 Wellington Street

Ottawa ON K1A ON4

Canada
Bibliothèque et

Archives Canada

Direction du

Patrimoine de l'édition

395 , rue Wellington

Ottawa ON K1A ON4

Canada
Your file Votre référence

ISBN: 978-0-494-91517-2

Our file Notre référence

ISBN: 978-0-494-91517-2
NOTICE:

The author has granted a nonexclusive license allowing Library and Archives Canada to reproduce, publish, archive, preserve, conserve, communicate to the public by telecommunication or on the Internet, loan, distrbute and sell theses worldwide, for commercial or noncommercial purposes, in microform, paper, electronic and/or any other formats.

The author retains copyright ownership and moral rights in this thesis. Neither the thesis nor substantial extracts from it may be printed or otherwise reproduced without the author's permission.
AVIS:

L'auteur a accordé une licence non exclusive permettant à la Bibliothèque et Archives Canada de reproduire, publier, archiver, sauvegarder, conserver, transmettre au public par télécommunication ou par l'Internet, prêter, distribuer et vendre des thèses partout dans le monde, à des fins commerciales ou autres, sur support microforme, papier, électronique et/ou autres formats.

L'auteur conserve la propriété du droit d'auteur et des droits moraux qui protege cette thèse. $\mathrm{Ni}$ la thèse ni des extraits substantiels de celle-ci ne doivent être imprimés ou autrement reproduits sans son autorisation.
In compliance with the Canadian Privacy Act some supporting forms may have been removed from this thesis.

While these forms may be included in the document page count, their removal does not represent any loss of content from the thesis.
Conformément à la loi canadienne sur la protection de la vie privée, quelques formulaires secondaires ont été enlevés de cette thèse.

Bien que ces formulaires aient inclus dans la pagination, il n'y aura aucun contenu manquant. 


\section{Abstract}

This work examines the development of an 8-bit analog to digital converter (ADC) using the comparator assisted binary search (CABS) based architecture. The CABS $\mathrm{ADC}$ is a hybrid structure between the Flash $\mathrm{ADC}$ and the successive approximation register (SAR) ADC, capable of achieving excellent energy per conversion in the order of femto Joules. The architecture relies on a post fabrication calibration strategy to correct for process variation tolerances (PVT) and establish comparator threshold levels.

From simulation results the 8-bit ADC is capable of achieving a resolution of 7.98 bits at $\mathrm{DC}$ with a maximum frequency of operation of $20 \mathrm{MHz}$ and an excellent figure of merit (FOM) of only $15.8 \mathrm{fJ}$ per conversion. The input range is $600 \mathrm{mV}$ differential, and the integral nonlinearity (INL) and the differential nonlinearity (DNL) are within $1 / 2 \mathrm{LSB}$. The effective resolution bandwidth (ERBW) achieved is $25 \mathrm{MHz}$ with a signal to noise and distortion ratio (SINAD) of $49.1 \mathrm{~dB}$ and a spurious free dynamic range (SFDR) of $66.3 \mathrm{~dB}$. The core power consumption without output latches and drivers is only $122 \mu \mathrm{W}$ for a sampling frequency of $20 \mathrm{MHz}$. The ADC was fabricated in a $0.13 \mu \mathrm{m}$ IBM CMOS eight metal layer process (CMRF8SF). The use of an external sample-and-hold (SAH) in the measurement phase of the fabricated ADC places an upper limit on the maximum frequency of operation of the ADC. The measured FOM at a sampling frequency of $25 \mathrm{MHz}$ is $15 \mathrm{fJ}$ with a core power consumption of $100 \mu \mathrm{W}$. 
Using a non-optimum calibration code the INL of the fabricated ADC was improved from $64 \mathrm{LSB}$ to $11 \mathrm{LSB}$. The DNL was improved from $-68 \mathrm{LSB}$ to $14 \mathrm{LSB}$. The ENOB was improved to 5.1 bits from 3.1 bits. The SINAD was $32.3 \mathrm{~dB}$ and the SFDR was $35.7 \mathrm{~dB}$ for an input frequency of $50 \mathrm{kHz}$ at a sampling frequency of $4 \mathrm{MHz}$. 


\section{Acknowledgments}

First and foremost I would like to express my sincere gratitude to my parents Constantin and Mihaela, as well as Irene and Miro for their continuous moral support during this challenging time. I'm fortunate to be surrounded by such loving people. As well I am indebted to Igor Miletic, Boris Spokoinyi, Kiril Kidisiuk, Stefan Shopov, Ivan Smojic, Vahidin Jupic, Nagui Mikhail for their technical support as well as constant encouragement. I would also like to express my gratitude towards Dr.Leonard MacEachern, for inspiring me, motivating me, as well as being a close

friend through the entire process. Through this endeavor I have learnt first hand of the challenges, the struggles and the elated feelings of success when an abstract concept is transformed into a functioning element in the physical world. The medium of communication in the information age is created in field of Electronics.

"The limits of my language means the limits of my world."

Ludwig Wittgenstein 


\section{Contents}

$\begin{array}{ll}\text { Abstract } & \text { iii }\end{array}$

$\begin{array}{lll}\text { Acknowledgments } & \mathbf{v}\end{array}$

Table of Contents $\quad$ vi

List of Tables $\quad$ x

List of Figures $\quad$ xii

Nomenclature $\quad$ xvii

1 Introduction $\quad 1$

1.1 Motivation ...................... 1

1.2 Thesis Organization . . . . . . . . . . . . . . . 2

2 Background 4

2.1 Contemporary ADC Architectures . . . . . . . . . . . . . . . 4

2.1.1 Flash ADC .................... 4

2.1 .2 Pipelined ADC .................... 6

2.1.3 Folding and Interpolating A/D ............. 6

2.1.4 Successive Approximation ADC . . . . . . . . . . . . . 8

2.1 .5 CABS ADC ..................... 10 
2.1.6 Comparison of Relevant Architectures _. . . . . . . . . 11

2.2 CABS ADC Target Specifications . . . . . . . . . . . 16

3 CABS Architecture $\quad 18$

3.1 System Overview . . . . . . . . . . . . . . . . . . 18

3.2 ADC Components . . . . . . . . . . . . . . . . . 25

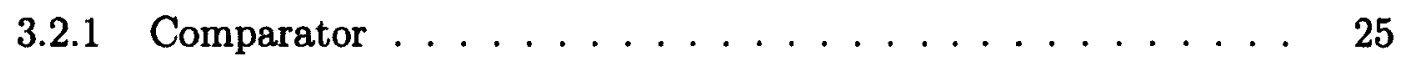

3.2 .2 MOSCAP Tuning Arrays $\ldots \ldots \ldots \ldots \ldots \ldots$

3.2.3 Shift Register Memory . . . . . . . . . . . . . . . . 26

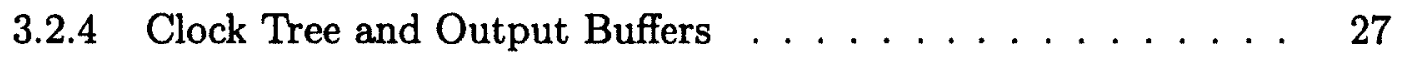

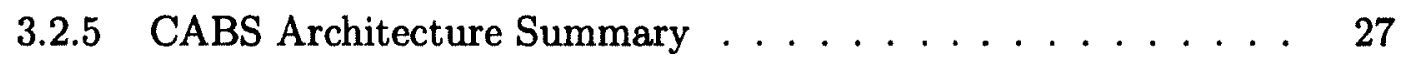

4 Design of CABS ADC 29

4.1 Comparator Architecture . . . . . . . . . . . . . . . . . 30

4.1.1 Comparator Transient Behavior . . . . . . . . . . . 30

4.1.2 Analysis of Comparator Delays . . . . . . . . . . . . 32

4.1.3 Offset and Threshold Configuration . . . . . . . . . 42

4.1 .4 Offset Tuning $\ldots \ldots \ldots \ldots \ldots$

4.1.5 Complete Comparator Schematic Devices Sizes _...... . 50

4.1 .6 Comparator Layout . . . . . . . . . . . . . . 53

4.2 Bitline Drivers . . . . . . . . . . . . . . . . 55

4.3 Switched Capacitance MOSFET Tuning Array (SCMTA) . . . . . . 59

4.3.1 Method of Capacitive Imbalance . . . . . . . . . . . . 59

4.3.2 MOSCAP Varactor Arrays . . . . . . . . . . . . 60

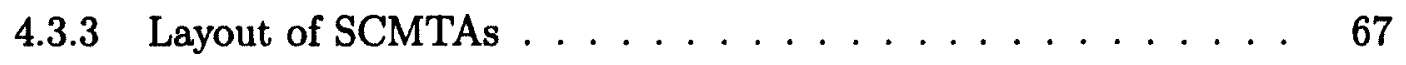

4.4 Shift Register Based Memory _ . . . . . . . . . . . . . . 69

4.4 .1 Shift Register Layout . . . . . . . . . . . . . . 71 
4.5 Output Buffer Design . . . . . . . . . . . . . . . . 73

4.5 .1 Output Buffer Layout $\ldots \ldots \ldots \ldots \ldots \ldots$

4.6 Clock Tree . . . . . . . . . . . . . . . . . . 76

4.6 .1 Clock Tree Layout $\ldots \ldots \ldots \ldots$. . . . . . . . . 78

4.7 ASIC Layout Integration $\ldots \ldots \ldots \ldots$

4.7 .1 Floor Planning $\ldots \ldots \ldots \ldots \ldots$

$4.7 .2 \quad$ 4-Bit Hard Coded Core . . . . . . . . . . . . . . 81

4.7.3 Routing Methodology . . . . . . . . . . . . . . . . . 82

4.7.4 Power Supply Lines and Decoupling Capacitors . . . . . . . 83

4.7.5 Layout Approach Summary . . . . . . . . . . . . 86

4.8 ADC Design Summary . . . . . . . . . . . . . . . . . . 88

5 ADC Metrics and Simulation $\quad 90$

5.1 Static Linearity Analysis . . . . . . . . . . . . . . . . 90

5.1 .1 Integral Nonlinearity $(\mathrm{INL}) \ldots \ldots \ldots \ldots$

5.1 .2 Differential Nonlinearity (DNL) $\ldots \ldots \ldots \ldots$

5.1.3 Effects of Netlist Complexity on Simulation Time . . . . . . 94

5.2 Dynamic Analysis . . . . . . . . . . . . . . . 96

5.2.1 Jitter Analysis of Dynamic Latched Comparator . . . . . . . 99

5.2 .2 Dynamic Analysis Metrics . . . . . . . . . . . . . . 101

5.3 Power Dissipation . . . . . . . . . . . . . . . . . 103

5.4 The Effects of ADC Design Choices on Static and Dynamic Performance Metrics . . . . . . . . . . . . . . . . 105

5.5 Summary of Simulation Results . . . . . . . . . . . . . . . 108

6 ADC Measured Results $\quad 111$

6.1 Test Configuration . . . . . . . . . . . . . . . . 112 
6.1 .1 Test Configuration $1 \ldots \ldots \ldots \ldots \ldots$

6.1 .2 Test Configuration $2 \ldots \ldots \ldots \ldots \ldots$

6.2 PCB Design . . . . . . . . . . . . . . . . . . 116

6.2 .1 Power Supply Decoupling _ . . . . . . . . . . . . . 116

6.2.2 Signal Integrity and Propagation $\ldots \ldots \ldots \ldots \ldots$

6.2.3 Preliminary PCB Design, "PCB1" . . . . . . . . . . . . 118

6.2.4 Secondary PCB Design with External SAH, "PCB2" . . . . . 123

6.3 Preliminary Testing . . . . . . . . . . . . . . . . . 130

6.4 Calibration . . . . . . . . . . . . . . . 134

6.5 Static Linearity Analysis . . . . . . . . . . . . . . . . . 136

6.5 .1 Integral Nonlinearity $(\mathrm{INL}) \ldots \ldots \ldots \ldots$

6.5.2 Differential Nonlinearity (DNL) $\ldots \ldots \ldots \ldots$

6.6 Measured Dynamic Analysis . . . . . . . . . . . . . . . . . 139

6.7 Power Dissipation . . . . . . . . . . . . . . . . . . 140

6.8 Summary of Measurement Results . . . . . . . . . . . . . . 143

7 Conclusion $\quad 146$

7.1 Contributions to Research . . . . . . . . . . . . . . 146

7.2 Future Work . . . . . . . . . . . . . . . 147

$\begin{array}{ll}\text { List of References } & 151\end{array}$

$\begin{array}{ll}\text { Appendix A Bill of Materials for PCB1 } & 157\end{array}$

$\begin{array}{lc}\text { Appendix B Bill of Materials for PCB2 } & 158\end{array}$ 


\section{List of Tables}

2.1 Comparison of published state-of-the art low power CMOS based ADC

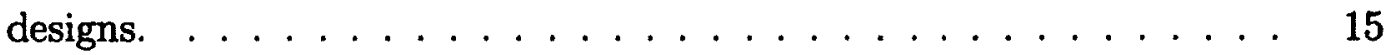

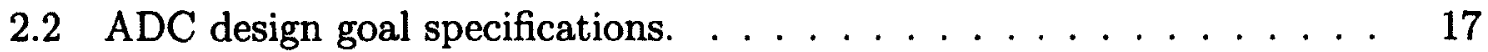

4.1 Device size specifications for the nominal comparator. . . . . . . . 50

4.2 Device size specifications for the input pairs of the comparators in the

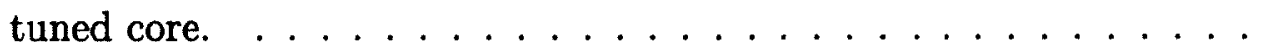

4.3 Device size specifications for the input pairs of the comparators in the hard-coded core. . . . . . . . . . . . . . . . . . 51

4.4 Device sizes for the output buffer. . . . . . . . . . . . . . . . 74

4.5 Input capacitance of WE-DFF $\ldots \ldots \ldots \ldots \ldots \ldots$

4.6 Maximum current tolerance for power supply nets. . . . . . . . . . 84

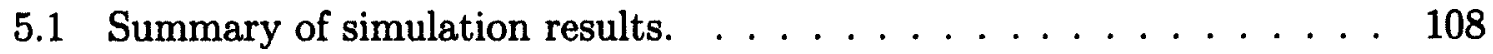

5.2 Comparison of simulated results to published state-of-the art low power

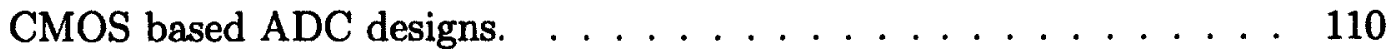

6.1 Test bench equipment. . . . . . . . . . . . . . . . . 113

6.2 Decoupling component device sizes. . . . . . . . . . . . . 117

6.3 Summary of measurement results for non-optimum calibration code. . 143

6.4 Comparison of measurement results to published state-of-the art low power CMOS based ADC designs. . . . . . . . . . . . 145 
A.1 Bill of materials for the preliminary PCB1 . . . . . . . . . 157

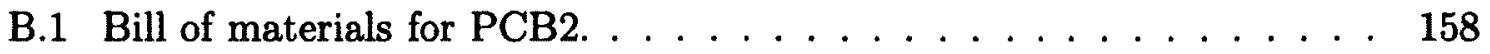




\section{List of Figures}

2.1 Conceptual schematic of a 3 bit Flash ADC . . . . . . . . 5

2.2 Conceptual structure for a Pipelined ADC architecture. . . . . . . 7

2.3 Conceptual schematic for a folding and interpolation ADC architecture. 7

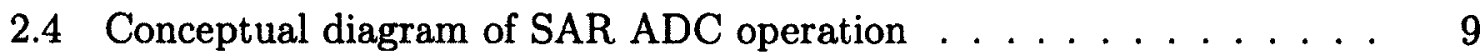

2.5 Conceptual schematic of a CABS ADC architecture. . . . . . . . 10

2.6 Comparison of frequency of operation as a function of the figure of merit for published ADCs. . . . . . . . . . . . . . 13

2.7 Comparison of ADC resolution as a function of the figure of merit for

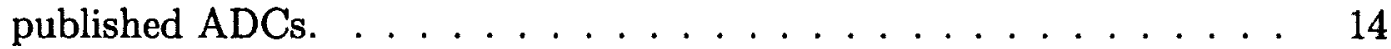

3.1 Root and children structure for a segment in the CABS structure . . 19

3.2 CABS hard-coded core. . . . . . . . . . . . . . . . . 20

3.3 Top level architecture for CABS ADC . . . . . . . . . . . 22

3.4 Architecture of a row macro cell. . . . . . . . . . . . . . 24

4.1 Comparator core without tuning arrays. . . . . . . . . . . . 31

4.2 Dynamic latched comparator transient waveforms for a low differential

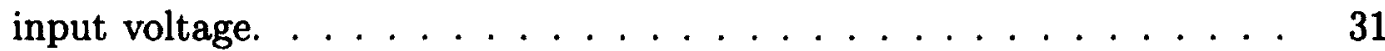

4.3 Transient response of the ADC through the positive 1 LSB path . . . 33

4.4 Simulation and theoretical total delay for a nominal comparator $\mathrm{V}_{\mathrm{os}}=0 \mathrm{mV}$ as a function of input voltage $\ldots \ldots \ldots \ldots$ 
4.5 Highest capacitance and lowest input voltage binary search paths . . 41

4.6 Monte carlo simulation for nominal comparator. . . . . . . . . . . 43

4.7 Standard deviation of the input offset as function of device width/length variation. . . . . . . . . . . . 46

4.8 Total comparison delay as function of device width/length variation. . 47

4.9 Offset as function of relative width imbalance. . . . . . . . . . 48

4.10 Full comparator schematic including tuning arrays. . . . . . . . . 52

4.11 Initial comparator layout for the nominal comparator. . . . . . . . 53

4.12 Final comparator layout for the nominal comparator. . . . . . . . 54

4.13 Positive threshold bitline driver. . . . . . . . . . . . . . 55

4.14 Comparators with connected bitline drivers and sampling falling edge D Flip-Flops . . . . . . . . . . . . . . . . . . . . . . . . 55

4.15 Transient simulation including bitline encoder and falling edge flip flop 57

4.16 Propagation delay as a percentage of half the duty cycle of the CLK

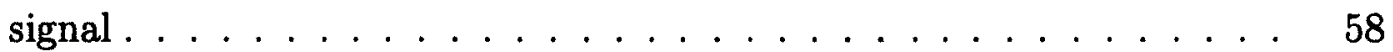

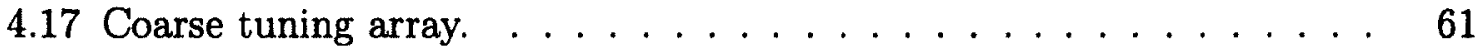

4.18 Fine tuning array tuning array. $\ldots \ldots \ldots \ldots 61$

4.19 Monte carlo simulation for the offset the nominal comparator. . . . . 63

4.20 Comparison delay and the minimum tuned threshold as a function of device length. . . . . . . . . . . . . . . 63

4.21 Contour plot of the capacitance of a MOSCAP (M6) device. . . . . 65

4.22 Threshold variation as a function of the coarse array tuning code. . . 66

4.23 SCMTA Layout . . . . . . . . . . . . . . . . 68

4.24 Shift register used for the control signal of the SCMTA tuning cell. . 69

4.25 Layout of the shift register . . . . . . . . . . . . . 71

4.26 Buffer test bench. . . . . . . . . . . . . . . . . 73 
4.27 Schematic diagram of output buffers. . . . . . . . . . . 74

4.28 Layout of the output buffer. . . . . . . . . . . . . . 75

4.29 Clock tree with three levels of corresponding hierarchy. . . . . . . 76

4.30 Clock tree layout. . . . . . . . . . . . . . . . . 78

4.31 Layout floor plan for the ADC. . . . . . . . . . . . . 80

4.32 Layout of the 4 bit HCC. . . . . . . . . . . . . . . . 81

4.33 Closeup of the routing channel layout. . . . . . . . . . . 82

4.34 Layout of power supply lines feeding row macro cells. . . . . . . . 83

4.35 ICGCUOCT, CABS based ADC full IC layout. . . . . . . . . . 86

5.1 Sampled output bits. . . . . . . . . . . . . . . . . . 91

5.2 Magnification of the ADC transfer function. . . . . . . . . . . . 92

5.3 Full transfer function of the ADC with an overlay of the transition code best fit line. . . . . . . . . . . . . . . . . 92

5.4 Integral nonlinearity (INL) as a function of input voltage $\ldots \ldots . . .93$

5.5 Differential nonlinearity (DNL) obtained using the difference between code transition $\ldots \ldots \ldots \ldots \ldots \ldots \ldots$

5.6 FFT spectrum of the input signal for $f_{s}=19.9680 \mathrm{MHz}$, $\mathrm{f}_{\mathrm{i}}=0.1365 \mathrm{MHz} . \ldots \ldots \ldots \ldots \ldots \ldots \ldots \ldots \ldots$

5.7 Reconstructed analog output using ideal DAC. . . . . . . . . . 99

5.8 SINAD and SFDR for a sampling frequency $\mathrm{f}_{\mathrm{s}}=20 \mathrm{MHz}$ as a function of the input frequency $\mathrm{f}_{\mathrm{in}} \ldots \ldots \ldots \ldots \ldots \ldots \ldots$

5.9 ENOB for a sampling frequency $\mathrm{f}_{\mathrm{s}}=20 \mathrm{MHz}$ as a function of the input

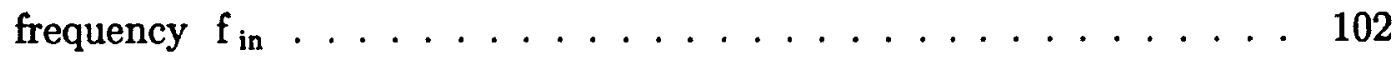

5.10 Power dissipation of the digital core. . . . . . . . . . 103

5.11 Power dissipation of the full IC. . . . . . . . . . . . . . . 104

5.12 FOM for the core power consumption. . . . . . . . . . 105 
5.13 Comparison of ADC resolution as a function of the figure of merit for

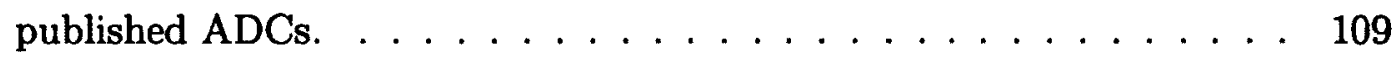

5.14 Comparison of frequency of operation as a function of the figure of merit for published ADCs for the design presented in this work. . . . 109

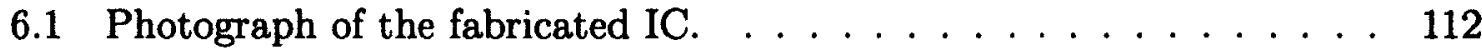

6.2 Photograph of the packaged IC . . . . . . . . . . . . 113

6.3 Functional diagram for preliminary test plan. . . . . . . . . . . . 114

6.4 Functional diagram for preliminary test plan with back-to-back configuration. . . . . . . . . . . . . . . . 115

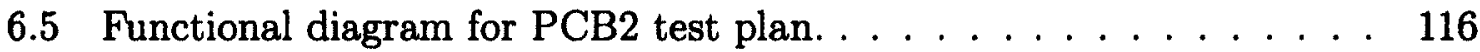

6.6 Selected area of the PCB showing griding between ground planes. . . 119

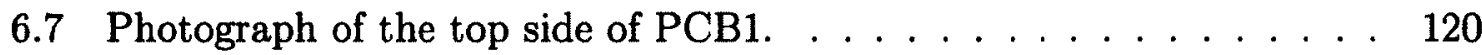

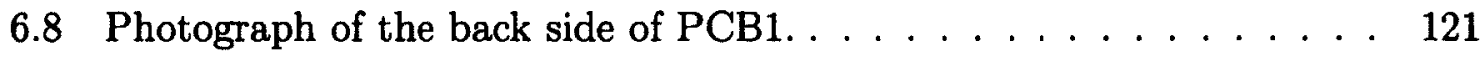

6.9 PCB layout, topside pictured left, bottom side pictured right. . . . . 122

6.10 PCB2 signal path $0 . \ldots \ldots \ldots \ldots \ldots \ldots$

6.11 PCB2 signal path $1 . \ldots \ldots \ldots \ldots \ldots \ldots \ldots$

6.12 PCB2 signal path $2 \ldots \ldots \ldots \ldots \ldots \ldots \ldots$

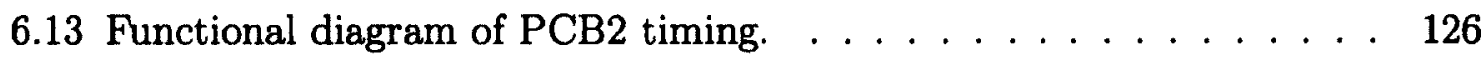

6.14 Layout of PCB2. . . . . . . . . . . . . . . . . . 128

6.15 Photograph of PCB2. . . . . . . . . . . . . . . . . . . 129

6.16 Screenshot from the DSO showing the ADC output bits. . . . . . . 130

6.17 Reconstructed output for fabricated ADC showing code transition sampling. . . . . . . . . . . . . . . . . . . . . 131

6.18 Reconstructed output for simulated ADC showing code transition sampling. . . . . . . . . . . . . . . . . . . . 132

6.19 Shift register programming of the fabricated ADC. . . . . . . 133 
6.20 Pre-calibration integral nonlinearity (INL) as a function of input voltage 136

6.21 Post-calibration integral nonlinearity (INL) as a function of input voltage 137

6.22 Pre-calibration differential nonlinearity (DNL) obtained using the difference between code transition $\ldots \ldots \ldots \ldots \ldots$

6.23 Post-calibration differential nonlinearity (DNL) obtained using the difference between code transition $\ldots \ldots \ldots \ldots$. . . . . . . 138

6.24 FFT spectrum of the input signal for $f_{s}=4 \mathrm{MHz}, f_{i}=50 \mathrm{kHz} . \ldots 139$

6.25 Total measured power consumption. . . . . . . . . . . . . . 140

6.26 Total measured power consumption for Nyquist operation. . . . . . 141

6.27 FOM for measured ADC power consumption. . . . . . . . . . 141

6.28 Measured ADC core power dissipation. . . . . . . . . . . . . . 142

6.29 Comparison of frequency of operation a function of the figure of merit

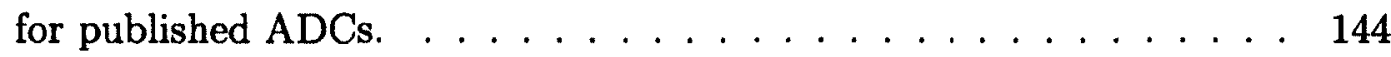

6.30 Comparison of ADC resolution as a function of the figure of merit for

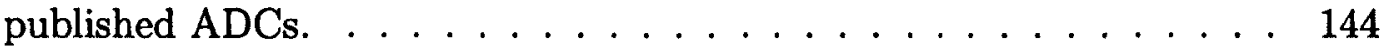

7.1 Functional diagram of SoC calibration of the CABS ADC. . . . . . 149 


\section{Nomenclature}

$\begin{array}{ll}\text { AC } & \text { Alternating Current } \\ \text { ADC } & \text { Analog to Digital Converter } \\ \text { AHDL } & \text { Analog Hardware Description Language } \\ \text { BJT } & \text { Bipolar Junction Transistor } \\ \text { CMC } & \text { Candian Microelectronics Corporation } \\ \text { CMOS } & \text { Complementary Metal Oxide Semiconductor } \\ \text { CS } & \text { Common-Source } \\ \text { DC } & \text { Direct Current } \\ \text { DAC } & \text { Digital to Analog Converter } \\ \text { DFT } & \text { Discrete Fourier Transform } \\ \text { DSP } & \text { Digital Signal Processing } \\ \text { DNL } & \text { Differential Non Linearity } \\ \text { DUT } & \text { Device Under Test } \\ \text { EM } & \text { Electromagnetic } \\ \text { ENOB } & \text { Effective Number of Bits } \\ \text { ESD } & \text { Electrostatic Discharge } \\ \text { FET } & \text { Field Effect Transistor } \\ \text { FFT } & \text { Fast Fourier Transform } \\ \mathrm{f}_{8} & \text { Sampling Frequency } \\ \end{array}$


$f_{i}$

IC

IDWI

INL

LSB

MOSFET

MSB

nFET

NOF

PCB

pFET

$\mathrm{RF}$

$\mathrm{SAH}$

SCMTA

SINAD

SFDR

SMA

SNR

SPI

SRF

THD

VDD

VEE
Input Frequency

Integrated Circuit

Intentional Device Width Imbalance

Integral Non Linearity

Least Significant Bit

Metal Oxide Field Effect Transistor

Most Significant Bit

n-Type Field Effect Transistor

Number of Fingers

Printed Circuit Board

p-Type Field Effect Transistor

Radio Frequency

Sample and Hold

Switched Capacitance MOSFET Tuning Arrays

Signal to Noise and Distortion Ratio (SNDR)

Spurious Free Dynamic Range

Subminiature Version A Connector

Signal to Noise Ratio

Serial Peripheral Interface Bus

Self-Resonant Frequency

Total Harmonic Distortion

Positive Supply Voltage (Also known as $\mathrm{V}_{\mathbf{s}}$ )

Negative Supply Voltage (Also know as VSS) 


\section{Chapter 1}

\section{Introduction}

\subsection{Motivation}

A trend in recent years has sought to bring more system components of a mixed signal environment into the digital domain. In a variety of electronic systems, the analogto-digital converter (ADC) is responsible for translating an analog signal (voltage or current) to a digital representation. As a consequence, there is a growing interest in ADC architectures which are tunable and treatable as digital building blocks offering maximum integration within highly affordable CMOS digital oriented processes. Some of these applications are in high-density mixed signal environments including but not limited to system-on-chip architectures (SoC), radio-frequency identification (RFID), implanted biomedical devices, sensor networks, low power radio frequency (RF) communication components, amongst others [1-3]. With a digital calibration back end it is possible to dynamically improve the performance metrics of the ADC through calibration at any point in the lifetime of the device allowing for maximum flexibility in the design of the ADC. 
Following the push towards ever decreasing transistor channel lengths with emerging digital oriented CMOS technology nodes, the performance of analog circuits typically decreases as the channel length shrinks $[4,5]$. Naturally the choice of circuit topologies can be used effectively to reduce the effects of device scaling at the expense of design complexity, but not eliminate those effects altogether. Overall the following trends are visible with a reduction in the minimum channel of a typical CMOS device; a higher transit frequency at the expense of decreased linearity, increase in the noise floor as the headroom shrinks, degradation of intrinsic gain ${ }^{1}$ as a consequence of the lower output resistance $[5,6]$. With all of these factors in mind it becomes evident that analog circuits and systems, such as the ADC, can benefit directly from calibration strategies. The objective of the design presented in this work is to extend the maximum resolution of the current state-of-the-art CABS architecture while maintaining a low FOM for the energy per conversion step.

\subsection{Thesis Organization}

This work begins with an overview of existing low power topologies in Chap. 2 along with the merits of the various architectures as well as the desired target specifications for the ADC designed herein. Chap. 3 contains a formal introduction to the CABS architecture, which is the architecture of choice for this femto Joule per conversion 8-bit ADC. Chap. 4 covers all aspects of the circuit level design. The design choices of various components as well as their relationships to system level specifications are discussed therein. In Chap. 5 static and dynamic ADC performance metrics are presented for the simulated ADC. In Chap. 6 the reader can find a discussion of the measured results as well as the test configurations used to extract them. Chap. 7

\footnotetext{
${ }^{1}$ Also referred to as the open-circuit gain, which is the product of the transconductance and the output resistance.
} 
concludes this work, summarizing the contributions to research as well as possible techniques to improve the performance of CABS type ADCs. 


\section{Chapter 2}

\section{Background}

\subsection{Contemporary ADC Architectures}

In the realm of $A D C$ design there exists a plethora of architectures intended for target applications requiring different specifications; high frequency, low power, small area, and high resolution. With the vast majority of metrics requiring opposing design practices, different architectures in ADC design are combined to negotiate between opposing specifications resulting in over all improved ADC performance. In the following sub sections, several architectures comparable in performance to the CABS ADC architecture are briefly discussed.

\subsubsection{Flash ADC}

Flash ADCs represent the faster branch of high speed ADC architectures [1]. In a Flash $\mathrm{ADC}, 2^{\mathrm{N}}-1$ comparators are connected in parallel for an $\mathrm{N}$ bit converter. $\mathrm{A}$ reference ladder ${ }^{2}$ is constructed to generate input voltages spaced one least significant bit (LSB) apart from each other. One LSB is defined as $\mathrm{V}_{\mathrm{REF}} /\left(2^{\mathrm{N}}\right)$. A conceptual diagram of the Flash architecture is presented in Fig. 2.1. The parallel comparators

\footnotetext{
${ }^{2}$ Resistive divider ladder.
} 
have the negative input node connected to their respective reference voltage and another to the common input voltage. When the clock edge rises all the comparators simultaneously enter the comparison phase.

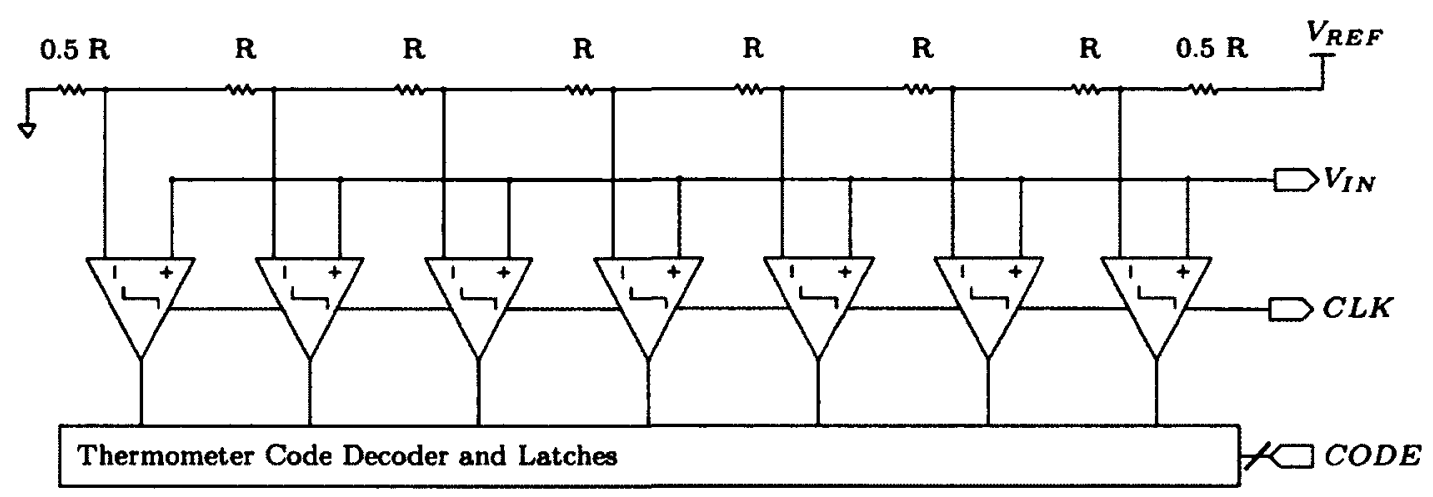

Figure 2.1: Conceptual schematic of a 3 bit Flash ADC. A 3 bit Flash converter requires $\mathrm{N}=2^{3}-1=7$ comparators. Comparators are simultaneously triggered resulting in a thermometer code which must be decoded into its binary representation.

If a common analog input voltage is higher than the reference voltage at the negative input of the comparator, the output is a logic " 1 " value. If the comparator falls within a certain reference bracket, all the comparator outputs below that comparator will result in logic " 1 " value and all the comparators above will result in a negative decision and a " 0 " logic value output. To extract the digital word, a thermometer code decoder is necessary to translate the direct output into its respective binary representation $^{3}$. The Flash ADC is typically used in applications requiring low to medium resolution, and high sampling frequencies. Increasing the resolution of a Flash ADC results in complex layout problems for the reference ladder. Each additional " $n$ " bit increases the number of devices by $2^{\mathrm{n}}$ resulting in a geometric increase in both the number of components and the power consumption. Recently more advanced Flash

\footnotetext{
${ }^{3}$ For a Flash ADC with a high resolution the decoder can become a significant bottleneck reducing the frequency of operation.
} 
structures have been introduced which eliminate the need for a reference ladder altogether [7-9] through the use of programmable, MOSFET based, capacitor's banks which shift the threshold of the comparators ${ }^{4}$.

\subsubsection{Pipelined ADC}

The Pipelined A/D converter splits the conversion into several stages. Each stage is responsible for converting $\mathrm{k}$ bits and consists of a $\mathrm{k}$ bit ADC converter in a back-toback configuration with a $\mathrm{k}$ bit ADC in one of the signal paths. The output of the $\mathrm{k}$ bit DAC is then subtracted from the sampled input. This residue is then amplified by $2^{k}$ bits in order to reuse the same stage design in the succeeding stage. From Fig. 2.2 several limitations become evident. It is prohibitive to assign a conversion higher than 2-3 per stage as the complexity and the limitations of the DAC and ADC in that stage begin to outweigh the benefits of pipelining. The Pipelined ADC also suffers from an increased latency ${ }^{5}$ and when used in a feedback configuration can lead to a critical decrease in performance $[1]^{6}$.

Pipelining is more of a concept than a specific architecture. ADCs within the stage can be constructed from Flash, Interpolating, Folding A/D converters with or without interpolation, depending on the most critical specification.

\subsubsection{Folding and Interpolating A/D}

The folding architecture performs its conversion through two parallel paths, fine and coarse conversion paths. The motivation behind this approach is to reduce the analogto-digital conversion latency experienced through a cascaded conversion path ${ }^{7}$ or

\footnotetext{
${ }^{4} \mathrm{By}$ shifting the thresholds of the comparators they act as a reference generator.

${ }^{5}$ As opposed to Flash, CABS structures.

${ }^{6} \mathrm{~A}$ feedback configuration such as Pipelined SAR ADC.

${ }^{7}$ Such as sub-ranging ADCs.
} 


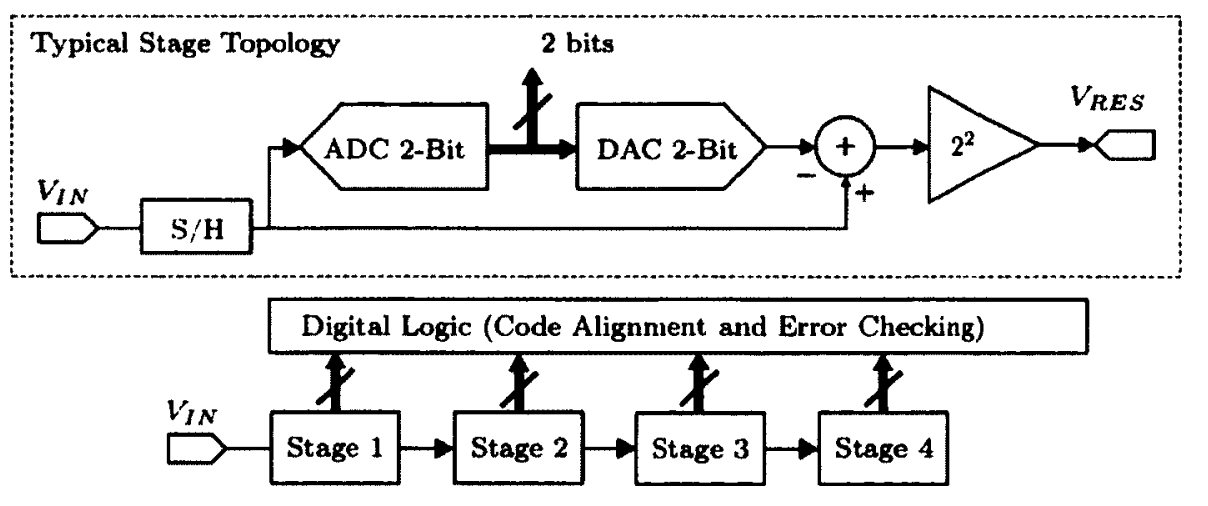

Figure 2.2: Conceptual structure for a Pipelined ADC architecture. The Pipelined $\mathrm{ADC}$ is built from back-to-back ADC/DAC blocks. The input of a sequential stage is the difference between the original and the reconstructed signal of the previous stage.

Pipelined ADC. The folder is typically constructed from cross coupled differential pairs in a parallel cascaded folding scheme. The input signal is then "folded" around $\mathrm{V}_{R E F} / 2$ and generates $2^{m}$ folding stages with an amplitude of $\mathrm{V}_{R E F} / 2^{m}$, where $m$ is the folding factor. This action is illustrated at the bottom of Fig. 2.3. Ideally the folding circuit requires a linear transfer function which is unattainable by standard CMOS technologies. For an $n$-Bit ADC the folder reduces the amount of comparators by $2^{m}$.

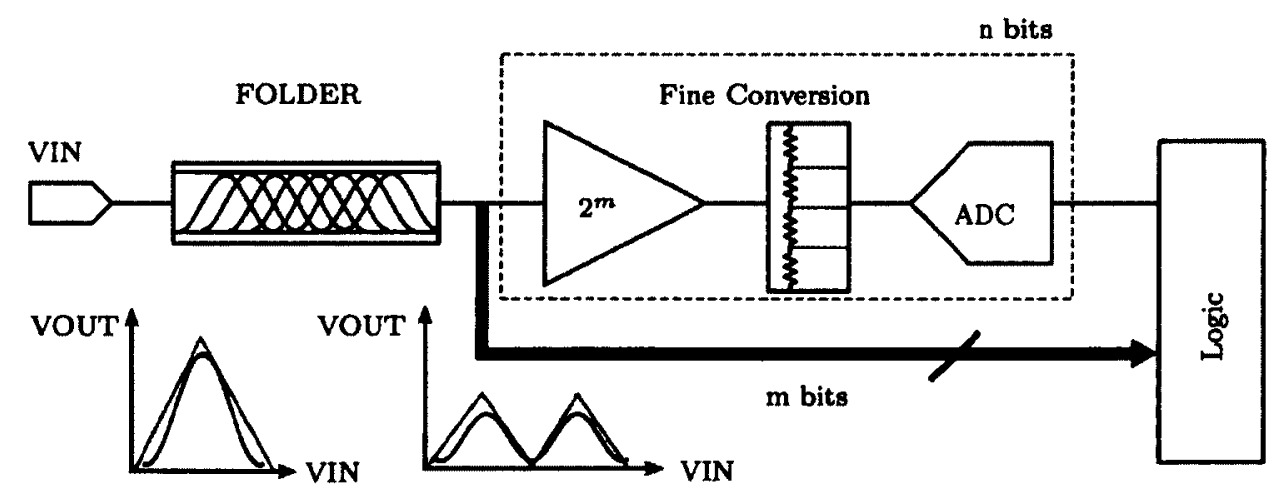

Figure 2.3: Conceptual schematic for a folding and interpolation ADC architecture. The folder wraps the input around $\mathrm{V}_{\mathrm{REF}} / 2$. Digital logic is then responsible for determining the folding region while a lower resolution $\mathrm{ADC}$ determines the remaining bits. 
A strategy used to alleviate this problem is to employ parallel folding circuits with a predetermined delay. The ADC could then be guaranteed to function in the linear region of operation of the folding circuit. The folder reduces the conversion range for the following stage but the proceeding stage does not know anything about the first most significant bits (MSBs) of the code. A "logic" stage is responsible for determining in which of those folding regions the fine conversion takes place along with performing error correction and synchronization. Through the fine conversion path the signal is amplified by $2^{m}$, to reduce matching constraints and maintain the same references [1]. An interpolation stage typically follows the folding stage. The interpolation is performed by resistive or capacitive voltage dividers and increases the resolution by some $k$ number of bits. After interpolation an "ADC", typically a simple flash ADC performs the final m-bit conversion. The resolution of the ADC is then given by $N=k+n+m$.

In industry grade applications presented in publications $[10,11]$ some sort of pipelining is used along with the folding and interpolation strategy to achieve a higher resolution. As the folding order is increased the size of the necessary coarse converter increases by the same amount. Thus a folding factor of 4 would require a 4-bit coarse converter. Taft et al. [10] eliminate the necessity of the parallel coarse channel path by extracting the coarse information with the addition of "distributed comparators" in the folding stages. Although this strategy reduces the overall number of comparators the reduction in parallelism increases the conversion latency.

\subsubsection{Successive Approximation ADC}

The successive approximation register (SAR) based ADC performs its conversion through a binary search lasting a minimum of $\mathrm{N}$ clock cycles for an $\mathrm{N}$ bit ADC. The SAR ADC employs a single comparator for the conversion process. This in turn, 
decreases both area and power consumption in comparison to other topologies [12]. In modern SAR topologies the DAC is created through a switched capacitor charge redistribution mechanism. The DAC is implemented by a binary weighted capacitor array. The required number of capacitors in the array is a minimum of $N+1$. As a consequence of the back-to-back configuration, and to guarantee that the DAC does not degrade the operation of the ADC, the DAC must have a higher number of bits. A conceptual system diagram of this topology is presented in Fig. 2.4

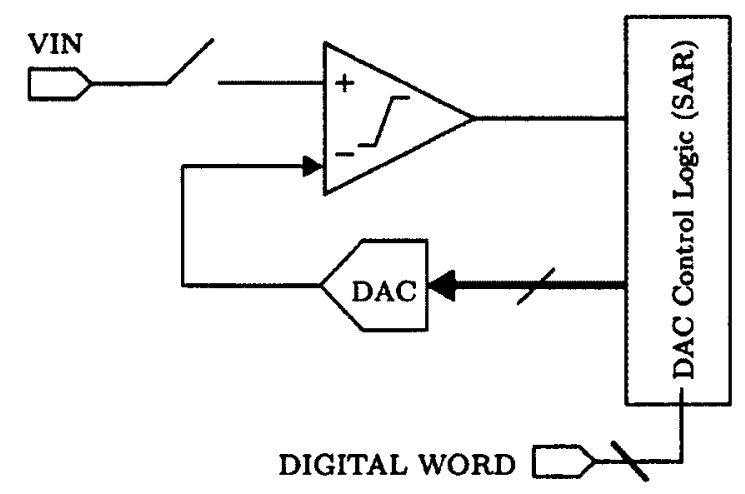

Figure 2.4: Conceptual diagram of SAR ADC operation. The SAR ADC performs a binary search, by adjusting the output value of the DAC through a control logic register. The SAR ADC requires $\mathrm{N}$ clock cycles for $\mathrm{N}$ bits and a higher resolution DAC.

In the first clock cycle, the DAC output voltage is in the middle of the ADC's linear input range, $\mathrm{V}_{R E F} / 2$. If the input voltage is larger, the MSB of the digital code is determined as a logic " 1 " with the proceeding bits being set to zero. In the second conversion, for the MSB - 1 bit, the DAC MSB is switched on along with the MSB - 1 of the DAC. The total value of the DAC is then $\mathrm{V}_{R E F} / 2+\mathrm{V}_{R E F} / 4$. Writing is enabled for the corresponding MSB-1 flip-flop in the register. Once the comparison takes place, the value is stored in the next clock cycle and the ADC proceeds to the next bit. Thus the SAR architecture performs an operation similar to a bisection search by halving the range around the approximate value in each successive comparison. 


\subsubsection{CABS ADC}

The CABS architecture is a recent addition to the world of $A / D$ converters, resembling a hybrid between the SAR ADC and the Flash ADC [13]. The conversion is realized through a comparator based assisted binary search (CABS) which is the same search algorithm performed during the operation of the SAR ADC. Unlike the SAR ADC the conversion takes place within one clock cycle similar to its Flash counterpart. The core of the CABS ADC is a binary tree consisting of comparators with built-in thresholds. The thresholds are established without the use of an external reference ladder and depending on the stage, combined with tuning circuitry to achieve the desired thresholds. The root comparator which represents the MSB bit is clocked by the global clock signal. Once it has reached a decision it asynchronously triggers one of its children.

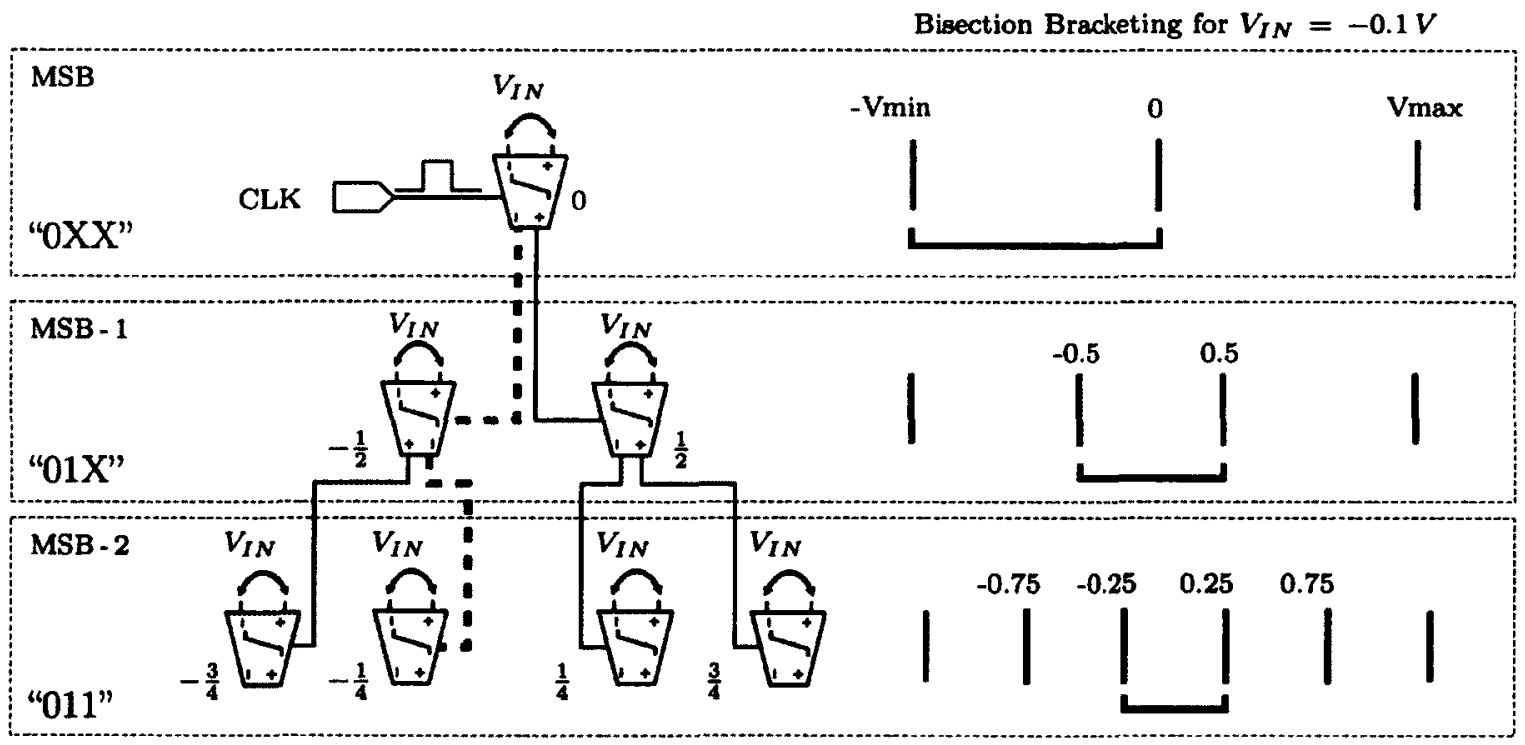

Figure 2.5: Conceptual schematic of a CABS ADC architecture. CABS is a SAR and Flash ADC, hybrid structure. The CABS ADC performs an asynchronous binary search in one clock period. Comparators are clocked by the outputs of comparators in a preceeding stage reducing conversion duration to one clock cycle. 
The example in Fig. 2.5 assumes a supply between $1 \mathrm{~V}$ for VDD and - $1 \mathrm{~V}$ for VSS and an example input voltage of $-0.1 \mathrm{~V}$. If the result of the MSB is " 0 ", as shown in Fig. 2.5, the comparator with a threshold of $-0.5 \mathrm{~V}$ is triggered in the second stage. In the third stage corresponding to MSB-2, the value of the example input is higher than the threshold of $-1 / 4 \mathrm{~V}$ of the comparator pulling up the positive output node of the comparator. Through this process the decision is bracketed by each successive stage from "0XX" for the digital word following the first stage to " $01 \mathrm{X}$ " after the second stage. The final digital word after the comparison in the third stage corresponds to "011".

Each stage has $2^{n-1}$ comparators $^{8}$ with a spacing between the thresholds of $\left(V_{M A X}-V_{M I N}\right) / 2^{n-1}$ (the LSB of the stage) where $V_{M A X}$ and $V_{M I N}$ represent the maximum, and minimum, of the input linear range. The CABS ADC has $2^{N}-1 \mathrm{com}-$ parators like the Flash ADC but only $\mathrm{N}$ of those comparators are activated during a conversion cycle leading to impressive power efficiency.

\subsubsection{Comparison of Relevant Architectures}

A comparison of the current state-of-the art ADC architectures described in this section is presented in Fig. 2.6. The sampling frequency of the ADC is presented as a function of the figure of merit (FOM). The figure of merit used as a metric for ADC performance is given by,

$$
E=\frac{P_{s w}}{2^{E N O B} f_{s}},
$$

where $(E N O B)$ is the effective number of bits, $P_{s w}$ is the average switching power of the $\mathrm{ADC}$, and $\mathrm{f}_{\mathrm{s}}$ is the sampling frequency. The figure of merit in Eq. 2.1 was

\footnotetext{
${ }^{8}$ Where the number of comparators in a stage is the same as for a flash ADC, effectively each stage requires a Flash $\mathrm{ADC}$ which has only comparator asynchronously triggered. In this discussion the first stage corresponding to MSB conversion is 1 , therefore the total number of comparators is $2^{\mathrm{n}-1}=1$.
} 
used in order to allow for a direct comparison to the work presented in [14] which is the architectural basis for this design. This figure of merit is also popular in journal publications describing state-of-the art ADCs, allowing a comparison between ADCs with different operating parameters such as sampling frequency, ENOB or power consumption performance.

For journal publications not reporting the ENOB the resolution of the ADC was used to calculate the FOM. The typical performance regimes of different architectures, discussed in this chapter, are readily visible in Fig. 2.6. Ideally an ADC should have an FOM as low as possible and $f_{s}$ as high as possible. SAR ADCs fall in the bottom left corner with low FOMs but at lower frequencies than other architectures. As well, some Folding and Pipelined ADCs achieve notable FOMs with the highest sampling frequencies. The poorest performance in terms of FOM is achieved by the power hungry Flash ADCs. The CABS architecture achieves better FOMs than any other architecture, falling in between SAR and Folding/Pipelined ADCs in terms of sampling frequency. The CABS architecture becomes an interesting option to achieve ultra low energy per conversion at mid range sampling frequencies.

The comparison between resolution and FOM in Fig. 2.7 brings another insight to the difference between ADC topologies. The trade off between resolution, sampling frequency, and FOM becomes more evident. Low power, low frequency SAR ADCs occupy the upper left corner of the plot. Flash ADCs have low resolution and high FOM trending towards the bottom right corner. Other topologies such as Folding and Pipelined are not very well correlated. Folding and Pipelined directly trade resolution for sampling frequency. A perfect example of this is [19] achieving high resolution and mid range FOM but at a much lower frequency than its Pipelined counterparts. In $\mathrm{Tb} .2 .1$ all the relevant performance metrics are presented for the designs presented 


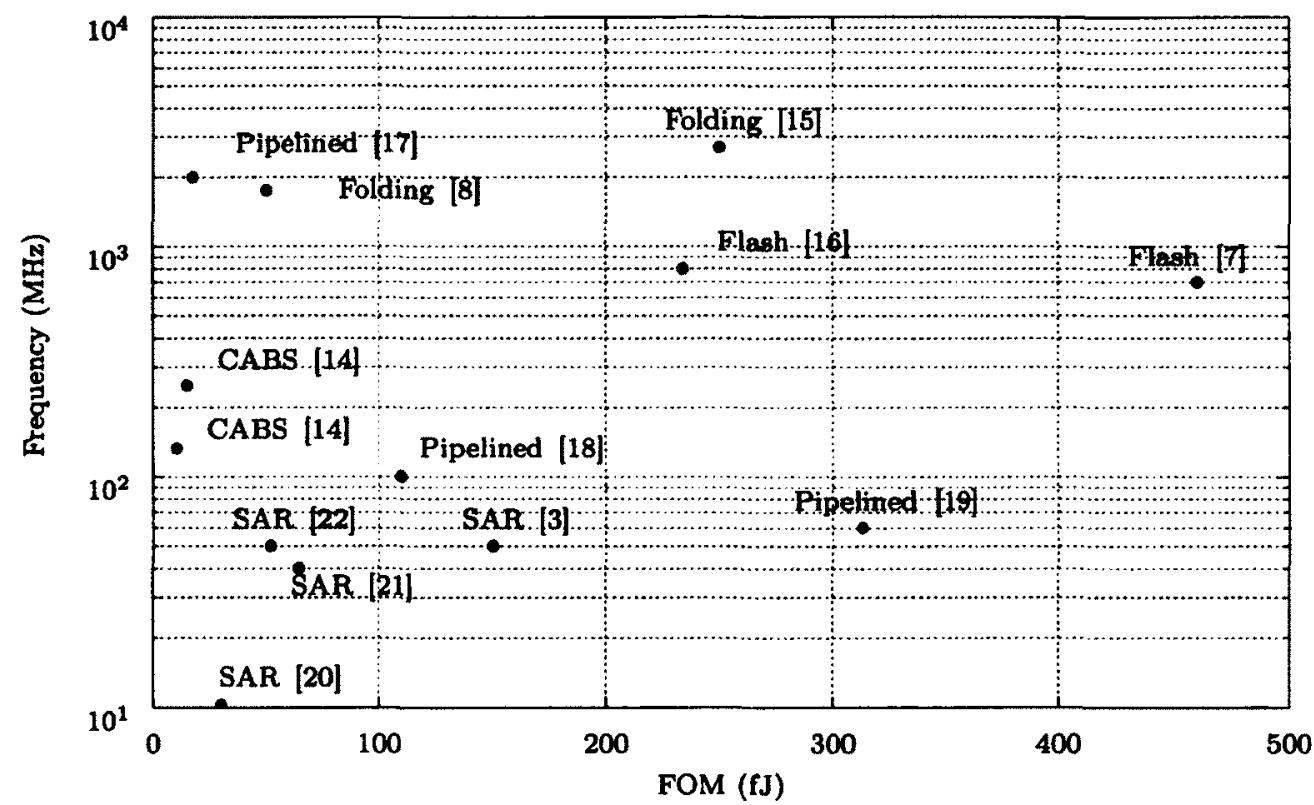

Figure 2.6: Comparison of frequency of operation a function of the figure of merit for published ADCs. For these examples $F O M=P_{s w} /\left(2^{\mathrm{ENOB}} f_{s}\right)$, where $P$ is average power consumption and $\mathrm{f}_{\mathrm{s}}$ is the sampling frequency.

in Fig. 2.6 and Fig. 2.7. Any unpublished data is specified by "NS". 


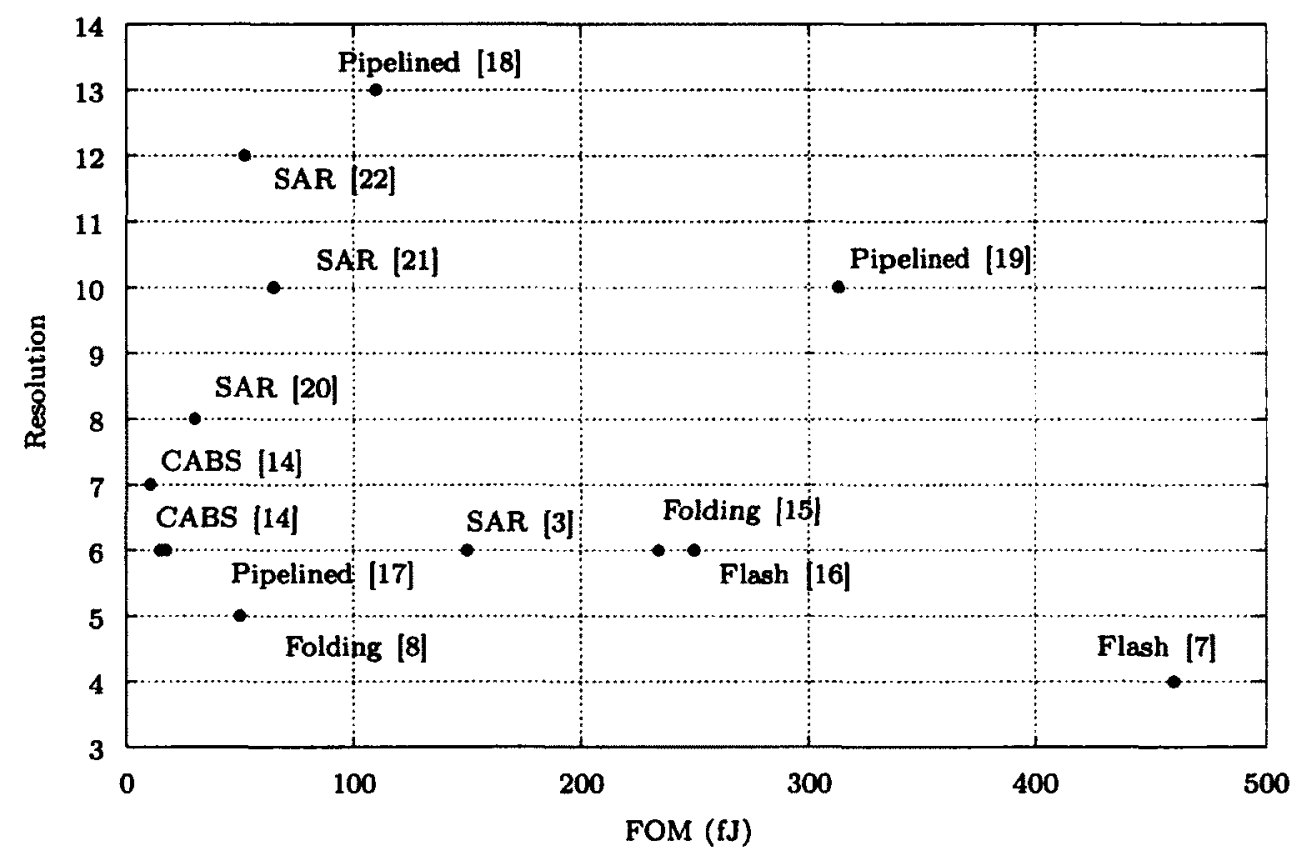

Figure 2.7: Comparison of $A D C$ resolution as a function of the figure of merit for published ADCs. For these examples FOM $=\mathrm{P}_{\mathrm{sw}} /\left(2^{\mathrm{ENOB}} \mathrm{f}_{\mathrm{s}}\right)$, where $\mathrm{P}$ is average power consumption and $f_{s}$ is the sampling frequency. 
Table 2.1: Comparison of published state-of-the art low power CMOS based ADC designs.

\begin{tabular}{|c|c|c|c|c|c|c|c|c|c|}
\hline Architecture & VDD & Technology & Area $\left(\mathrm{mm}^{2}\right)$ & Power $(\mathrm{mW})$ & Speed (MS/s) & Input Range (mV,diff) & Resolution & ENOB & FOM \\
\hline CABS [14] & $1 \mathrm{~V}$ & $90 \mathrm{~nm}$ & 0.055 & 0.133 & 133 & 768 & 7 & 6.4 & 10.4 \\
\hline Folding [15] & iv & $90 \mathrm{~nm}$ & 0.360 & 50 & 2700 & 800 & 6 & NS & 250.0 \\
\hline Flash [7] & $1.8 \mathrm{~V}$ & $0.18 \mu \mathrm{m}$ & NS & 4.43 & 700 & NS & 4 & 3.8 & 460.0 \\
\hline Pipelined [18] & $1.3 \mathrm{~V}$ & $0.13 \mu \mathrm{m}$ & 1.200 & 91 & 100 & 2000 & 13 & NS & 110.0 \\
\hline Pipelined [19] & $1.2 \mathrm{~V}$ & $0.13 \mu \mathrm{m}$ & 0.980 & 19.2 & 60 & NS & 10 & NS & 313.0 \\
\hline SAR [3] & $1 \mathrm{~V}$ & $90 \mathrm{~nm}$ & 0.055 & 0.24 & 50 & 320 & 6 & 5.0 & 150.0 \\
\hline SAR [20] & $1 \mathrm{~V}$ & $90 \mathrm{~nm}$ & 0.132 & 0.069 & 10 & 400 & 8 & 7.8 & 30.0 \\
\hline SAR [21] & $1 \mathrm{~V}$ & $65 \mathrm{~nm}$ & 0.060 & 1.21 & 40 & 1000 & 10 & 8.9 & 65.0 \\
\hline
\end{tabular}




\subsection{CABS ADC Target Specifications}

A summary of target specifications for the 8-bit CABS ADC design presented in this work can be found in $\mathrm{Tb}$. 2.2. The overall design goal was to improve the resolution of the CABS ADC while maintaining the same low FOM. In that respect the target specifications do not reflect any particular application or communication standard.

The desired input range was chosen after some preliminary simulations used to determine highest achievable comparator threshold. A larger tuning range increases the dynamic range of the $\mathrm{ADC}$ and lowers the noise floor. Unlike [14] the design was implemented in a $0.13 \mu \mathrm{m}$ technology node as opposed to $90 \mathrm{~nm}$. With a design implemented in an older technology node the target sample frequency is lowered in comparison to [14]. The increase in the resolution of the ADC also yields a decrease in the sampling frequency as will be explained with detail in the following chapters. Static analysis performance metrics such as the INL and dynamic performance metrics such as the SINAD are based on the performance of an ideal 8-bit ADC.

The integral nonlinearity (INL) and the differential nonlinearity (DNL) describe the monotonicity of the ADC transfer function as well as the code integrity ${ }^{9}$. The signal to noise and distortion ratio (SINAD) ${ }^{10}$, spurious free dynamic range (SFDR), and the effective number of bits (ENOB) are found through AC testing of the ADC ${ }^{11}$. The target SINAD is the SINAD of an ideal 8-bit ADC.

\footnotetext{
${ }^{9} \mathrm{An}$ INL and DNL less than $0.5 \mathrm{LSB}$ guarantees there are no missing codes.

${ }^{10}$ Also referred to as SNDR.

${ }^{11}$ These metrics describe the performance of $\mathrm{ADC}$ for different sampling or input frequencies.
} 
Table 2.2: ADC design goal specifications.

\begin{tabular}{cc}
\hline Metric & Simulation Value \\
\hline Technology & IBM CMRF8SF 130 nm \\
VDD & $1.2 \mathrm{~V}$ \\
Input Range & $600 \mathrm{mV}_{\text {pk-pk,diff }}$ \\
Sample Rate & $80 \mathrm{MHz}$ \\
Area & $2 \mathrm{~mm}^{2}$ \\
INL & $<0.5 \mathrm{LSB}$ \\
DNL & $<0.5 \mathrm{LSB}$ \\
SINAD @ Nyquist & $49.9 \mathrm{~dB}$ \\
SFDR @ Nyquist & $65 \mathrm{~dB}$ \\
ENOB @ DC & 8 \\
Power & $150 \mathrm{\mu W}$ \\
FOM & $10 \mathrm{fJ}$ \\
\hline
\end{tabular}




\section{Chapter 3}

\section{CABS Architecture}

This chapter offers a comprehensive overview of the CABS architecture, while being independent of any specific CMOS implementation technology e.g. IBM $130 \mathrm{~nm}$. As such it does not discuss in depth design details which are presented in Chap. 4. A system overview is presented in Sec. 3.1 detailing the conversion process for the CABS ADC. A brief discussion of some of the key components can be found in Sec. 3.2.

\subsection{System Overview}

The CABS ADC can be described as subtle hybrid between the Flash ADC structure and the SAR ADC [13]. The CABS ADC performs its conversion through the same strategy as the SAR ADC, a binary search, but within the span of only one clock cycle. The fundamental mechanism of conversion is carried out by a binary tree of comparators. The output nodes of a root comparator are connected to the clock inputs of the branch comparators. As the conversion propagates asynchronously through the binary tree, the quanta representing the input is bracketed within a shrinking range which halves at each succeeding stage. This predominant pattern of the CABS binary tree is illustrated in Fig. 3.1. 


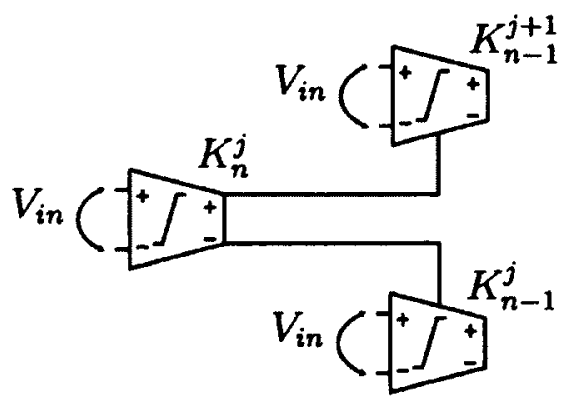

Figure 3.1: Root and children structure for a segment in the CABS structure, " $n$ " is the stage from 0 to 7, " $\mathrm{j}$ " is the comparator in a stage from 1 to $2^{\mathrm{n}}$.

For each comparator " $K$ " the subscript " $n$ " denotes the stage of the ADC and " $j$ " denotes the index of a particular comparator in a stage and " $N$ " is the resolution of the ADC. The generic equation for the offset of a comparator is given by

$$
V_{o s}\left(K_{n}^{j}\right)=(j) \cdot \frac{\left(V_{\max }-V_{\min }\right)}{2^{N-n}}+V_{\min }
$$

where

$$
j \in\left\{1,2^{N-n}\right\}, n \in\{7,0\} .
$$

For an 8-bit CABS ADC the first comparator corresponding to the first stage has an index $\mathrm{K}_{7}^{1}$. The first stage corresponds to the MSB conversion therefore the stage index is kept the same as the bit conversion index which is in big endian notation. This is a change in notation from the brief introduction offered in Chap. 2 but necessary to maintain the same notation as [14].

As an exercise to further investigate the conversion mechanism of the CABS ADC, the structure of a 4 bit comparator tree is presented in Fig. 3.2. Just like its Flash counterpart, all comparators in the binary search tree are connected to the same sampled input voltage. The only comparator connected to the global CLOCK signal is the root comparator representing the MSB. Through the entire conversion process only one comparator is asynchronously triggered in each stage leading to efficiencies 
of femto Joules per conversion [14].

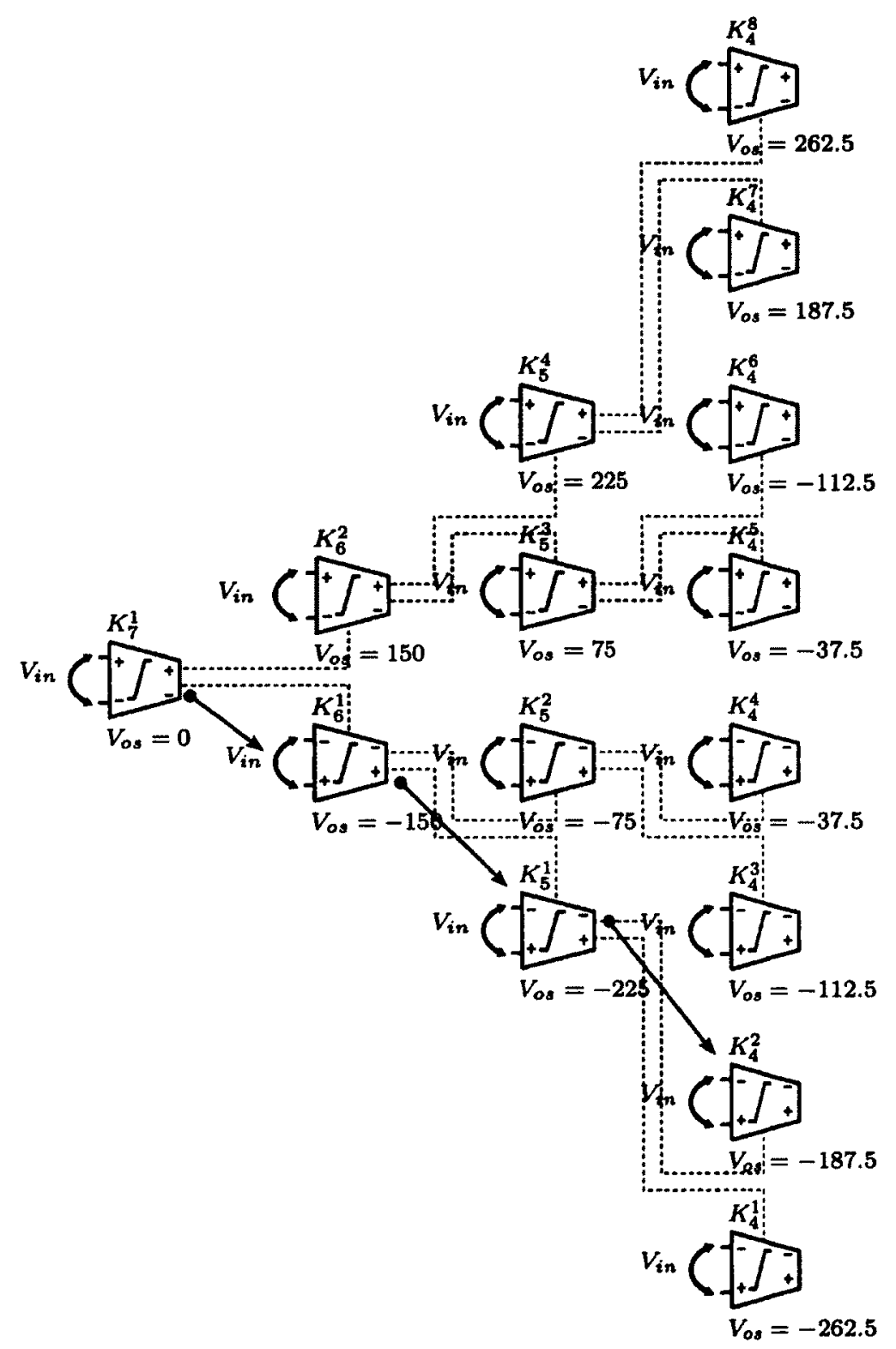

Figure 3.2: CABS hard-coded core (HCC). The asynchronous binary search path is illustrated by the directional arrows. This path assumes a differential input of $-190 \mathrm{mV}$ and a differential input range of $600 \mathrm{mV}$. The thresholds are calculated using Eq. 3.1.

Supposing a 4 bit CABS ADC is constructed with the specifications described in Sec. 2.2 we examine the conversion process in Fig. 3.2. The supply has a fixed value of 
$1.2 \mathrm{~V}$ and the linear input range is $300 \mathrm{mV}$ centered around midrail. The comparators used in the binary tree have a differential topology so the total linear range of the $\mathrm{ADC}$ is $600 \mathrm{mV}$ differential between $300 \mathrm{mV}$ and $-300 \mathrm{mV}$. This example assumes a differential input of $-190 \mathrm{mV}$.

On the positive edge of the global CLOCK signal, the root comparator $\left(K_{7}^{1}\right)$ begins the conversion process. Since the built in reference voltage of $\left(K_{7}^{1}\right)$ is 0 the comparison results in a logic " 0 " on the positive output, causing the negative output node of the comparator to rise to logic " 1 " value and a converted value of $0 \mathrm{XXX}$. The negative output node of $\left(K_{7}^{1}\right)$ is connected to the asynchronous clock input of $\left(K_{6}^{1}\right)$ which has a threshold of $-150 \mathrm{mV}$. Notice that the inputs of the comparators in the bottom half of the tree are inverted to generate the negative threshold. As a consequence the operation of the comparators is itself inverted and logic " 1 " output on the negative node of negative threshold comparator represents a positive "1" bit result in the binary search. As soon as $\left(K_{6}^{1}\right)$ is triggered it performs its comparison resulting logical " 1 " value on the positive output node and value of $00 \mathrm{XX}$ for the binary word, triggering $\left(K_{5}^{1}\right)$. The comparator $\left(K_{5}^{1}\right)$ has a threshold of $-225 \mathrm{mV}$ resulting in a logic " 1 " on the negative output and triggering the $\left(K_{4}^{2}\right)$ comparator which has an offset of $-187 \mathrm{mV}$ resulting in another negative output and the final converted word of 0011. Through this process the unsigned binary code is generated, starting from the MSB and proceeding to the LSB.

The first four MSB bits of the digital word are resolved by comparators with built in reference voltages and no tuning capability. The elimination of a tuning mechanism reduces the propagation delay through the first four stages, improving the operating frequency of the ADC, same as the design in [14]. The first four bits are termed the "hard-coded core" (HCC), in the CABS structure which is identical to the example described above. This distinction is made in Fig. 3.3 which shows the HCC and the 
tuned core (TC). In Fig. 3.3, "CAL" is the calibration clock for the shift register (SR), "WE" is the write-enable input for the SR, "D" is the data input. Separate clock trees are used for the aforementioned signals.

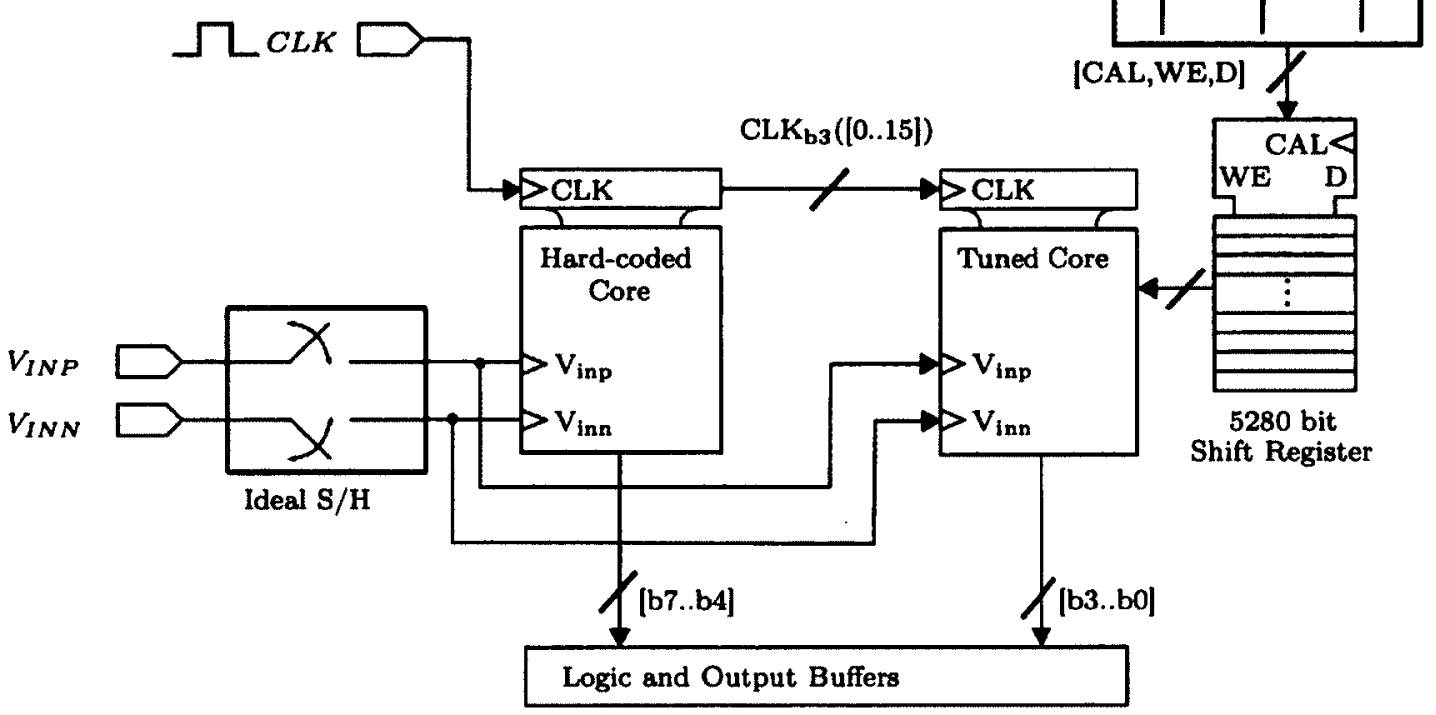

Figure 3.3: Top level architecture for CABS ADC. HCC (hard coded core), TC (tuned core), $\mathrm{CLK}_{\mathrm{b} 3}$ is a 16 bit bus carrying the asynchronous clock signals for stage 5 in the TC, "CAL" is the shift register (SR) calibration clock, "WE" is the SR write-enable signal, " $D$ " is the SR data input

The hard coded core has a total of $2^{4}-1$ or 15 comparators. The complexity of the architecture grows geometrically with each additional stage (bit). The amount of comparators in each stage starting with the MSB is $\langle 1,2,4,8,16,32,64,128\rangle$ for a resolution of 8 bits. This geometric increase in complexity becomes the fundamental limitation of the CABS structure. For a full Flash architecture, the geometric increase in complexity leads to a fundamental limitation when the size of the LSB approaches the PVT variations in the reference voltage ladder. For a full Flash A/D converter a 
maximum of $10-11$ bits is achievable without trimming [2].

Even with the inherent tuning capability of comparators, the CABS structure reaches the same limitation as the Flash structure for the maximum number of comparators in a particular stage. Each extra bit requires an additional, asynchronously triggered stage. This increases the conversion time and reduces the operating frequency. As the stages successively trigger during the high phase of the main clock, each proceeding stage has a shorter duration output pulse. If the frequency is increased, the short duration of a comparator output pulse is not capable of driving the clock input of the latter stage comparator resulting in a "pulse fading effect". In other words, one or more of the final stages of the ADC may never be asynchronously triggered. For reasons best left for discussion in Sec. 4.1 the comparison time of an LSB input can take $2 \mathrm{X}-3 \mathrm{X}$ longer than a full scale input further degrading the frequency of operation.

The CABS tuned core continues the tree structure from the hard-coded core but employs comparators which have both built in reference voltages and tuning capabilities. The motivation for introducing tuning capability in the latter stages of the ADC comes from process variation tolerance (PVT) during the fabrication of the chip. Monte Carlo simulations discussed in Sec. 4.1.3 confirm that the $3 \sigma$ variation of the offset approaches the LSB of the $4^{\text {th }}$ stage representing bit b4. From then on it becomes absolutely necessary to use tuning and calibration to correct the unintentional threshold shift resulting from PVT. Without calibration the INL and DNL of the ADC would degrade severely rendering the conversion meaningless. In addition to PVT, operating temperature will affect the INL and DNL. An optimum tuning code at room temperature is not guaranteed to ensure the same INL and DNL performance at a higher temperature e.g. $140^{\circ} \mathrm{C}$. The ADC could then be tuned at that 


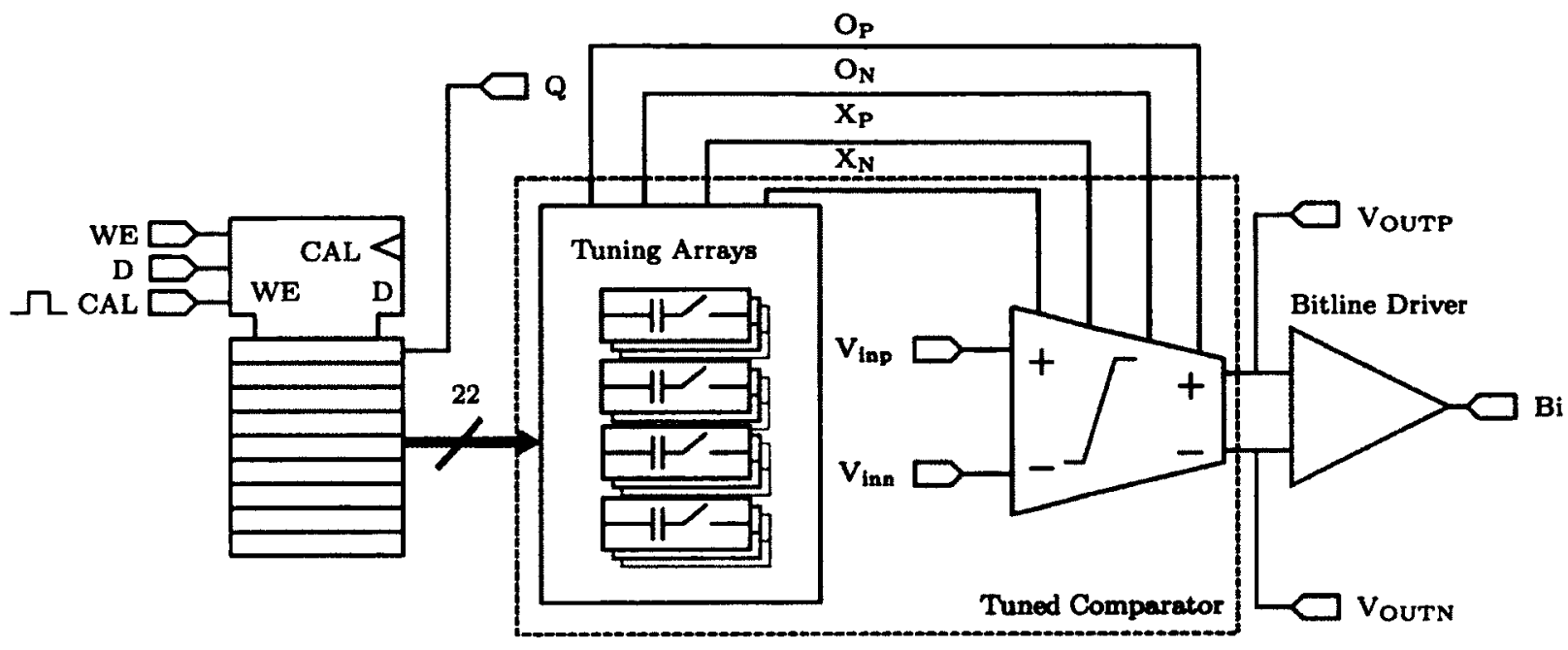

Figure 3.4: Architecture of a row macro cell, comparator, together the shift register, the tuning circuit (SCMTA), and the bitline driver form a "row macro cell". "Q" is the final element in the SR.

operating temperature or use a look up table to correct for a variation in the operating temperature. As well, over the life time of the ADC the operation of CMOS devices and interconnect begins to degrade. For this reason the ADC could be recalibrated at any point in its lifetime. The final bits, bit3 to bit0, require a total of $\left(2^{N}-1\right)-\left(2^{m}-1\right)=240$ comparators where " $\mathrm{m}$ " is the total number of bits converted by the hard coded stage and " $\mathrm{N}$ " is resolution of $\mathrm{A} / \mathrm{D}$ converter. The tuned core is programmed via a 5280 bit shift register which requires individual clock trees for the "WE" and "CLK" signals.

Comparators in the tuned core require additional circuitry to shift the threshold to the desired nominal value. This additional circuitry along with the comparator comprise a "tuned comparator" macro cell ${ }^{12}$ presented in Fig. 3.4. Tuning is accomplished by arrays of switched pFET-based MOSCAP capacitors discussed further in Sec. 3.2.2. There are four arrays of MOSCAPs connected to the internal nodes of the

\footnotetext{
${ }^{12}$ Also referred to as a "row macro cell" when including the SR.
} 
comparator which are indicated as " $\mathrm{X}_{\mathrm{N}}$ ", " $\mathrm{X}_{\mathrm{P}}$ ", " $\mathrm{O}_{\mathrm{P}}$ ", " $\mathrm{O}_{\mathrm{N}}$ " in Fig. 3.4.

The MOSCAP arrays are programmatically enabled by a serial-in parallel-out shift register. A bitline driver is responsible for assigning the correct bit for the comparison in the stage correctly for both positive and negative threshold comparators. The output of every bitline comparator in a particular stage is connected to the same node. The bitlines are sampled by a falling edge D flip-flop, on the falling edge of the global clock. The result of the conversion then becomes available to the output buffers.

\subsection{ADC Components}

\subsubsection{Comparator}

The comparators used for both the hardcoded core, and the tuned core, are based on the dynamic latched Strongarm comparator used in [14,23]. The structure was originally introduced by Kobayashi [24] as a sense amplifier based flip-flop for use in VLSI design of low power, high speed, SRAM memories. A differential voltage is applied to the input of the comparator. The result of a positive comparison results in a logic " 1 " on the positive output node and logic " 0 " on the negative output node of the comparator, the opposite being true for a negative comparison.

As previously discussed the comparator suffers from PVT effects which cause the built in threshold to shift from the nominal values when the IC is fabricated. The built in thresholds are realized by creating an intentional imbalance in the input differential pair of the comparator. To allow for both a correction of PVT effects on the device, and to achieve the desired offset, it is necessary to make use of arrays of pFET-based MOSCAP capacitors. Utilizing comparator calibration is a common technique in ADC design. It would be difficult to use auto-zeroing techniques or 
offset reduction techniques since the comparator's threshold is not centered around mid-rail but intentionally imbalanced. Such offset reducing techniques could require a separate reference ladder, multiple CLK cycles for a single conversion, and increased power consumption.

\subsubsection{MOSCAP Tuning Arrays}

Precise tuning of the comparator threshold is accomplished through the use of switched MOSCAP arrays. The pFET MOSCAP has both the source and drain terminals tied together and connected to the output of a WE flip-flop (WE-FF). The gate of a MOSCAP is then tied to the comparator's internal nodes. The tuning arrays are responsible for correcting both PVT induced offset shift and bringing the threshold to within a fraction of an LSB from the desired nominal value. Two types of arrays are implemented; a coarse array with 7 binary scaled devices creates 7 bits of tuning or 128 possible capacitance values as well as a 4 element fine tuning array with linearly scaled devices. When a MOSCAP is activated it creates a capacitance imbalance on either the negative or the positive output side of the comparator. As a consequence the opposing output node charges faster and a time delay is transformed into an output referred input voltage offset shifting the threshold of the comparator.

\subsubsection{Shift Register Memory}

The tuning array described in Sec. 3.2.2 require 7 bits for a coarse tuning array and 4 bits for a fine tuning array on each side of the comparator. This leads to a requirement of 22 bits which are accessed in parallel. Each comparator and its accompanying circuitry are organized in the "tuned row" macro cell presented in Fig. 3.4. The shift register is created out of 22 sequential write-enable D flip-flops 
(WE-DFFs). The flip flops are in turn constructed from a mux and d-latches built from transmission gates. The total size of the shift register for the tuned core is $\mathbf{5 2 8 0}$ bits which are addressed in parallel at all times during the operation of the ADC, unless the ADC is in the calibration mode of operation.

\subsubsection{Clock Tree and Output Buffers}

The large shift register required for the parallel tuning code raises several complications. To guarantee the integrity of the tuning code, the shift register clock signal must arrive at approximately the same time at each flip-flop. This is also the case for the WE signal, although the requirement is less stringent since the WE signal is enabled high for at least 5280 clock periods of the SR clock signal while the tuning code is loaded. Three levels of hierarchy were used in the design of the clock tree. At the bottom of the hierarchy, one custom buffer drives 8 rows of macro cells. At the second level one buffer is capable of driving 5 identically sized buffers. At the top hierarchy one buffer drives the final three children and associated interconnects. The buffers used in the clock tree are also used to implement the output buffers of the ADC. Once fabricated the IC is packaged and placed on a custom designed printed circuit board (PCB). In order to drive the off chip load impedance it is necessary to use output buffers. Additionally the output buffers isolate the bitline drivers from the external environment to the IC.

\subsubsection{CABS Architecture Summary}

In this chapter an introduction to the CABS ADC architecture was presented without reference to any specific CMOS process or technology node. In Sec. 3.1 the predominant pattern of the CABS ADC was introduced. Only the MSB comparator 
is triggered by the global clock signal. Comparators in the MSB-1 stage are triggered asynchronously by the output of the MSB comparator. This "root and children" structure, propagates through the entire CABS tree creating the mechanism which implements the binary search algorithm. The first four MSB bits are resolved by a hard coded core which features comparators with built-in reference voltages, reducing the conversion time through those stages.

The last four bits use tuning to compensate for threshold shifts in the comparators (unintentional offset) as a result of PVT as well as implementing the desired thresholds for those comparators. The tuning is achieved through switched pFET-based arrays of MOSCAPS as discussed in Sec. 3.2.2. In the hard coded core, comparators and bitline drivers are lumped together in a "row macro cell". Similarly for the tuned core, the pFET-based MOSCAP tuning arrays, shift register memory, comparator, and bitline driver comprise a "tuned row macro cell". The shift register based memory used to program the pFET-based MOSCAPS was introduces in Sec. 3.2.2. Compared to [14] two additional bits are added to the tuned core. As will be discussed in Chap. 4 the addition of two tuned bits further increases the layout area and design complexity. Each tuned row macro cell requires 22 bits of parallel memory to program the switched MOSCAPS. For the entire tuned core it was found that, 5280 bits are required to program the entire tuning code of the ADC. Finally, the chapter concluded in Sec. 3.2.4 with a brief introduction to the clock trees used to drive the large shift register as well as the output buffers necessary to drive off-chip loads. 


\section{Chapter 4}

\section{Design of CABS ADC}

In this chapter the full design process of an 8-bit CABS based ADC is explored. The chapter starts with an introduction of the fundamental building block of the CABS ADC, the dynamic latched comparator, in Sec. 4.1. The method used to establish variable thresholds for the comparators is discussed in depth in Sec. 4.3. The memory bank used to store the calibration code is examined in Sec. 4.4. Components necessary to drive a large external off-chip load as well as large capacitive loads on the $\mathrm{IC}^{\mathbf{1 3}}$, such as output buffers and the clock tree are presented in Sec. 4.5 and Sec. 4.6. Finally the layout design and integration process is discussed in Sec. 4.7.

All design simulations used the IBM $0.13 \mu \mathrm{m}$ CMOS BSIM4v4 MOSFET models and performed using Cadence Spectre v10.1, part of the IC 6.15 Cadence Tools suite. All simulations were performed on a server with the following specifications:

- Processor: Dual Intel(R) Xeon(R) CPU W5580

- Memory: 24 Gigabytes Triple Channel DDR2 PC 12000

- Hard Disk: Raid 5 - Intel-SSD 64G

\footnotetext{
${ }^{13}$ Such as several rows of tuned comparators
} 


\subsection{Comparator Architecture}

Throughout Sec. 4.1 the various aspects of comparator behavior and design are presented in a systematic way. Sec. 4.1.1 offers an insight into the dynamic behavior of the comparator. In section Sec. 4.1.2 two approaches are presented in analyzing the delays of the dynamic latched comparator. In Sec. 4.1.3, the knowledge gained from Sec. 4.1.2 is used to an advantage in creating built-in thresholds for the comparators. Finally Sec. 4.1.4 presents the tuning mechanism used to shift the threshold of the comparators.

\subsubsection{Comparator Transient Behavior}

During the reset phase CLK $=0$, transistors M7-M10 in Fig. 4.1 find themselves in the saturation region of operation behaving as closed switches. In turn they pull the nodes $\mathrm{X}_{\mathrm{N}}, \mathrm{X}_{\mathrm{P}}, \mathrm{O}_{\mathrm{P}}$ and $\mathrm{O}_{\mathrm{N}}$ down to the GND. At the same time, the inverter pair M11 and $\mathrm{M} 12$, pulls the common node $\mathrm{V}_{\mathrm{CM}}$ down to GND. In this, the low phase of the CLK signal, the comparator is effectively turned off with no current flow through any branches. The behavior of the core comparator during a comparison can be broken down into three separate phases analogous to the presentations in $[23,25]$. During the first phase $A$, the node $V_{C M}$ is quickly pulled to VDD by the inverter pair M11 and M12. This begins to push M1 and M2 into the saturation regime of operation. The nodes $\mathrm{X}_{\mathrm{N}}$ and $\mathrm{X}_{\mathrm{P}}$ are then linearly charged as shown in Fig. 4.3. During this phase the cross coupled inverters are not active. In this example a positive differential voltage of $150 \mathrm{mV}$ is applied at the input. The differential signal applied to the input transistors cause an imbalance in the current flow through the two branches. Transistor M1 is turned on harder than $M 2$ charging the node $X_{P}$ faster than the node $X_{N}$. 


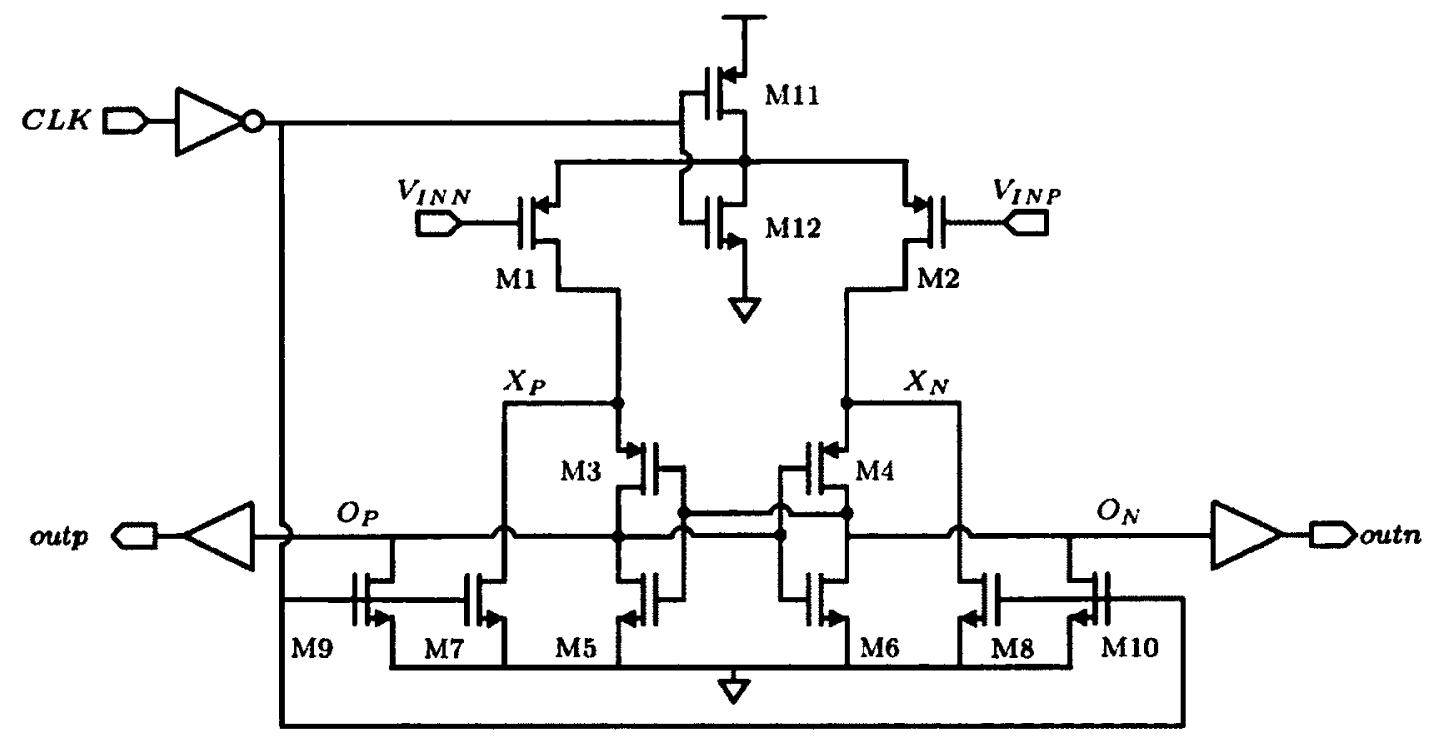

Figure 4.1: Comparator core without tuning arrays.

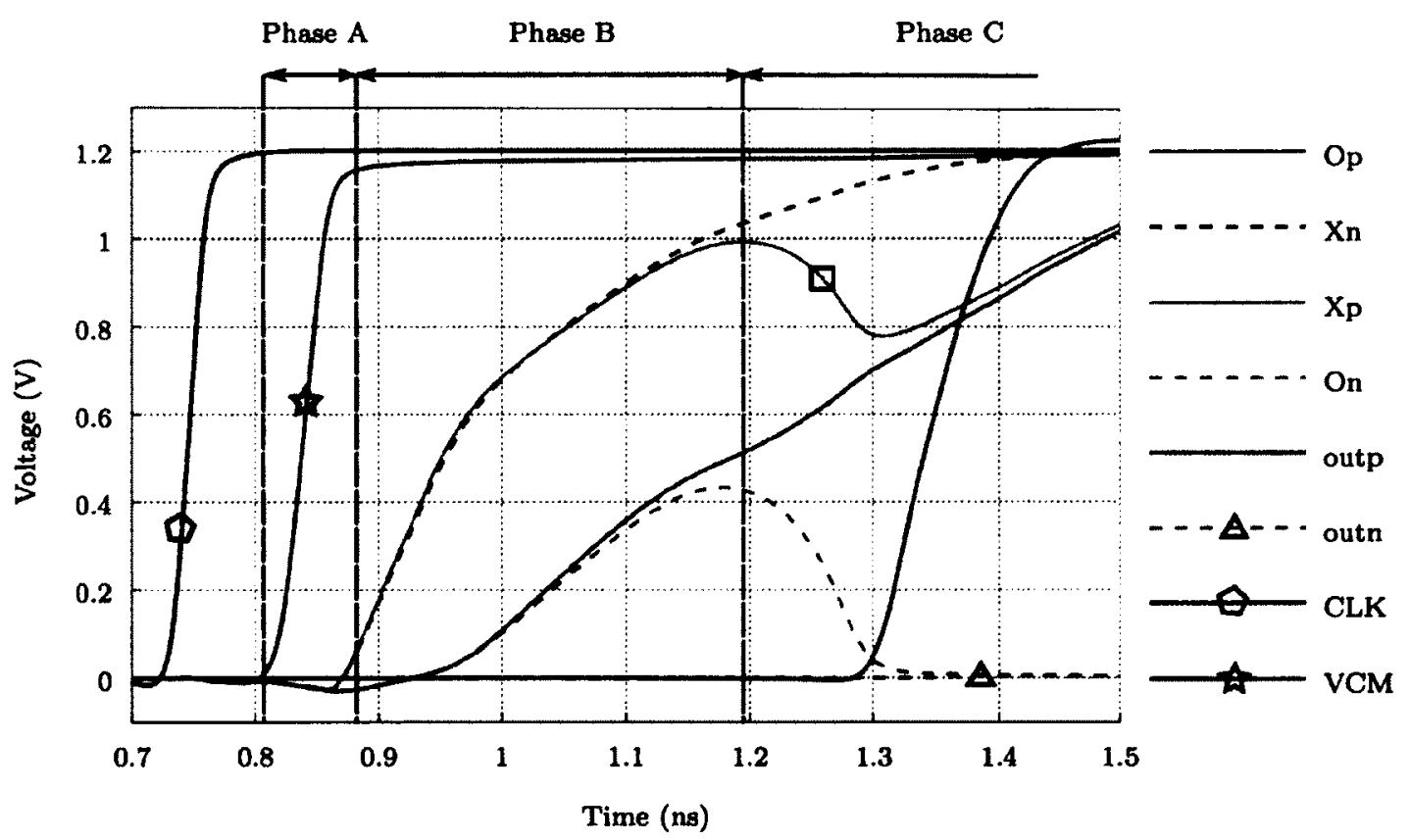

Figure 4.2: Dynamic latched comparator transient waveforms for a low differential input voltage.

The second phase, "B", is characterized by the activation of the positive feedback inverters in the dynamic latch. As nodes $X_{N}$ and $X_{P}$ are pushed towards VDD (throughout the 3 phases), the pFET transistors M3, M4 in the positive feedback 
latch begin turning on. This point is approximately given by $V_{t h, M 3}$ which corresponds to $0.591 \mathrm{mV}$ in this example. When the transistors start conducting the imbalance between the voltages at the nodes $\mathrm{X}_{\mathrm{N}}$ and $\mathrm{X}_{\mathrm{P}}$ is further reinforced. During this phase the nodes $O_{N}$ and $O_{P}$ are charged linearly.

In the third phase " $\mathrm{C}$ ", the input transistors leave the saturation region of operation and enter triode. As M5 and M6 enter the saturation region, the strong positive feedback action of the cross coupled inverters amplifies the regeneration nodes, bringing the comparison closer to the final logic value. During this phase transistors M4 and M5 begin turning off, reducing the current sinked to zero. During this phase node $\mathrm{O}_{\mathrm{N}}$ is slowly pulled back down to zero. This marks the end of phase $\mathrm{C}$ as node $\mathrm{O}_{\mathrm{P}}$ reaches the $V_{t}$ of the buffer driving the final logic output of the comparator to positive "1".

\subsubsection{Analysis of Comparator Delays}

The total duration for a comparison is a critical system level consideration which ultimately determines the maximum operating frequency of the CABS ADC. As can be seen in Fig. 4.3, the duration of the output pulse shrinks as the comparison propagates with a domino effect through the eight stages of the ADC. It is therefore of great interest to analyze the delays involved in the comparison process along with their governing factors.

Several analyses of the comparison time $e^{14}$ of the dynamic latched comparator have appeared in publications over the last few years. Some are based on empirical modeling [25] while others are based on a combination of simulation and large

\footnotetext{
${ }^{14}$ Also referred to as the "total delay" or "total comparison delay".
} 


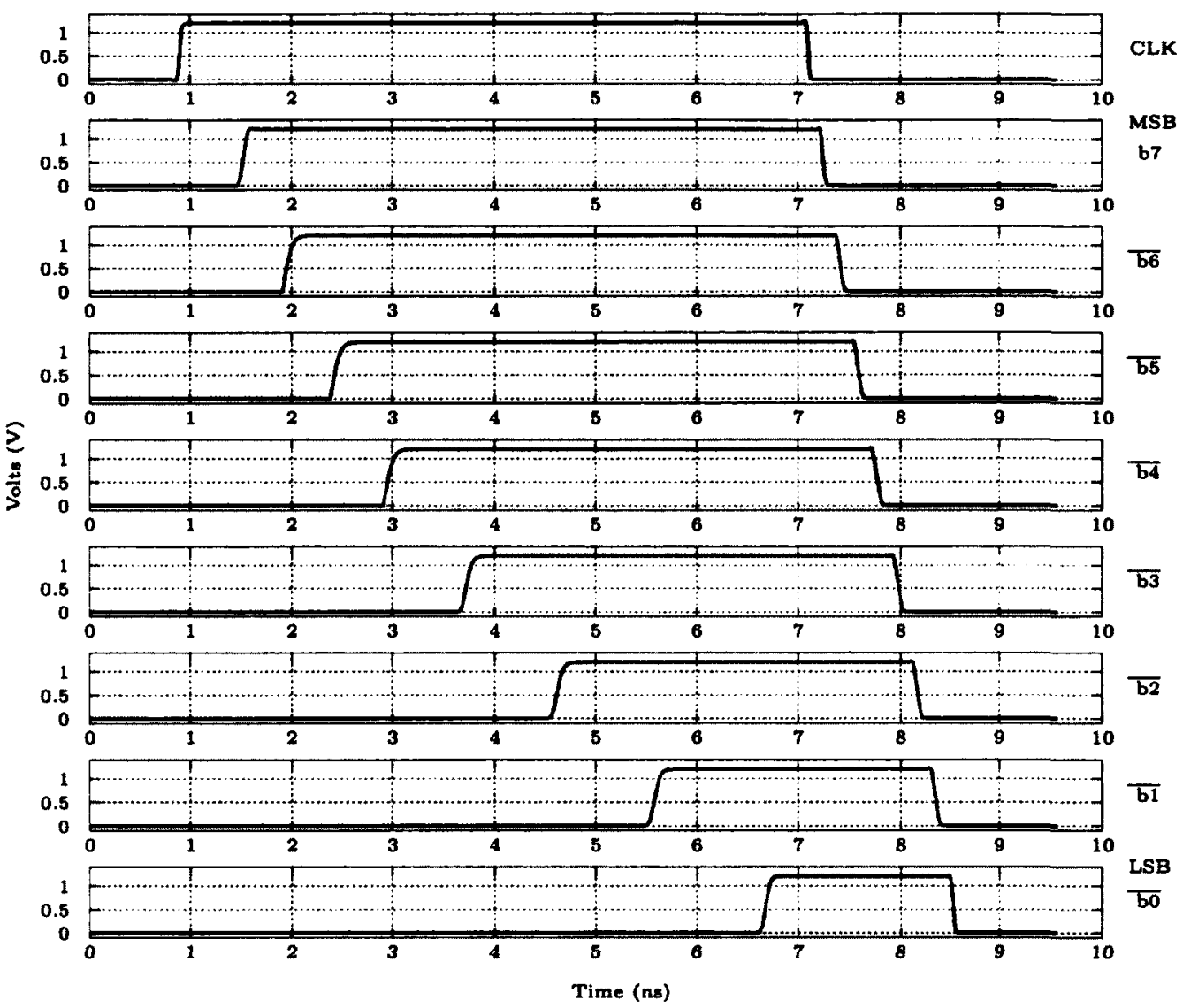

Figure 4.3: Transient response of the ADC through the positive $1 \mathrm{LSB}$ path, $\mathrm{f}_{\mathrm{S}}=$ $80 \mathrm{MHz}$,differential $\mathrm{V}_{\mathrm{IN}}=0.8 \mathrm{LSB}$, for an output code of $\mathrm{B}=10000000$ from top to bottom the signals are CLK,MSB ... LSB

signal analysis $[23,26,27]$. Both approaches are useful in understanding the dependence of the total delay on circuit parameters. Although this section does not describe the threshold configuration mechanism presented in Sec. 4.1.3 it is important to note that using threshold adjustment increases the load on the regeneration nodes $\mathrm{O}_{\mathbf{P}}$ and $\mathrm{O}_{\mathrm{N}}$. Using threshold adjustment results in an increase in the total delay of the comparator ${ }^{15}$. Optimizing comparator device sizes for reduced total delay and designing the SCMTA $^{16}$ threshold tuning circuits or bitline drivers ${ }^{17}$ must

\footnotetext{
${ }^{15}$ Any increase in the total delay reduce the sampling frequency of the ADC.

${ }^{16}$ MOSFET tuning arrays are used to establish tuned thresholds and are discussed in Sec. 4.3.

${ }^{17}$ Bitline drivers encode the correct bit decision, presented in Sec. 4.2.
} 
be performed concurrently as a "comparator macro-cell"18. Throughout the design discussion, design metrics or delay paths are described in terms of units of LSB given by Eq. 4.1. Since the ADC presented in this work has an input range of $600 \mathrm{mV}$,diff, $1 \mathrm{LSB}=600 / 256=75 / 32 \mathrm{mV}$.

$$
L S B=\frac{V_{\text {input,range }}}{2^{N}}
$$

To capture the behavior of the dynamic latched comparator it is necessary to solve the differential large signal equations at the boundaries of the "set-up" and "regeneration" phases. This has been carried out by Nikoozadeh, and Murmann in [27] as well as Plas et al. [23], amongst others. The following delay for the total comparison is presented in [23], where $C_{o}$ is the capacitance at the output nodes, $g_{d s}$ is the drain to source conductance and $g_{m}$ is the transconductance of the specified transistor,

$$
\tau_{R}=\frac{\left(g_{d s 1}+g_{m 3}\right) C_{o}}{g_{d s 1}\left(g_{m 3}+g_{m 5}\right)+g_{m 3} g_{m 5}} .
$$

During the regeneration phase ${ }^{19}$, the largest contribution in Eq. 4.2 is from $g_{d s 1}$, the output conductance, (as the input transistors M1-2 enter their linear region of operation $)^{20}$ and the equation reduces to Eq. $4.3[27]$ :

$$
\tau_{R}=\left.\frac{C_{o}}{\left(g_{m 3}+g_{m 5}\right)}\right|_{g_{d s 1} \gg g_{m 3}, g_{m 5}}
$$

Although the transconductance of the devices in the latch is lowered by short

\footnotetext{
${ }^{18} \mathrm{~A}$ macro-cell consists of a row which includes the following; comparator, bitline driver, SCMTA, 22 bit shift register memory.

${ }^{19}$ The regeneration phase refers to Phase B in Sec. 4.1.1.

${ }^{20}$ The input differential pairs enter the triode region.
} 
channel effects and dependence on non-linear bias points ${ }^{21}$, some logical conclusions can be drawn. Larger devices in the inverter latch will lower the overall latching delay by some factor of $\alpha=\sqrt{W /(\Delta W+W)}$, where $\mathrm{W}$ is the width, lowering the total delay.

In turn the parasitic capacitance and the loading capacitance due to the source of M3 and the drain of M5 slightly increases the contribution to the load capacitance at the nodes $O_{P, N}$ by some factor of $1 / \alpha^{2}$. As a consequence the delay in the set-up phase or "slew-phase", Phase A, increases as the time to discharge the regeneration nodes increases. Evidently there is an optimum size for the devices in the feed-back latch for any choice of input differential pair device size width. The input differential size could be scaled up to neutralize the effect of the increase in capacitance and lower the delay of the slew phase. This is done at the expense of power consumption and layout real estate.

Threshold tuning is established through switched MOSFET based capacitors connected to the regeneration and output nodes. The tuning method is explored with more detail in Sec. 4.3. In reference to the discussion above, the size of the devices used to establish the configurable thresholds must be scaled by the same factor as the differential pair and/or the inverter latch to reduce the total delay ${ }^{22}$. Each node utilizes seven binary scaled devices, required to alleviate PVT variations and establish the desired thresholds. The largest device in the tuning arrays has a size of $128 \mathrm{~W}_{\min }$ or $20.48 \mu \mathrm{m}$. As well, the total delay of the comparator would increase with larger tuning devices increasing the capacitance on the regeneration nodes.

\footnotetext{
${ }^{21}$ Ignored by the approximations in Eq. 4.3.

${ }^{22}$ This approach is carried to maintain the same threshold tuning range.
} 
In [25], Wicht presents an empirical analysis of the delays in the dynamic latched comparator. Fundamentally, the total comparison delay can be divided into two separate, physically meaningful delays ${ }^{23}$. The approach is different from $[14,27]$ where the operation of the comparator is divided into the same respective regions, but differential equations are used to find approximate expressions for the delay. Please note that for consistency the notation from [25] has been adjusted to the same notation used in [14].

The total delay for a comparison is given by

$$
t_{d}=t_{o}+t_{\text {latch }}
$$

where $t_{0}$ corresponds to the duration of Phase A which is the "set-up" delay or the slew-delay. Physically, it reflects the duration necessary to turn on the first nFET in the inverting latch which is the total time to discharge nodes $X_{N}$ and $X_{P}$. The second delay $t_{\text {latch }}$ is the total duration required for the cross coupled inverter pair to latch the input and is given by

$$
t_{\text {latch }}=\frac{C_{o}}{g_{m, e f f}} \ln \left(2 \frac{\Delta V_{\text {out }}}{V_{o}}\right)
$$

where the output voltage $\Delta V_{\text {out }}$ is the threshold of the output buffer connected to the output node. Assuming that we are operating the comparator within the linear input range the latching duration is linearly proportional to the capacitance on the output nodes $\mathrm{O}_{\mathrm{P}}$ or $\mathrm{O}_{\mathrm{N}}$ through $\mathrm{C}_{\mathrm{o}}{ }^{24}$. The latching delay also depends on the voltage

\footnotetext{
${ }^{23}$ The final stage Phase $\mathrm{C}$ is not significant, by this point the comparator has already reached a decision.

${ }^{24}$ This example assumes that the differential input pairs are have the same parameters.
} 
difference $V_{o}$, between the nodes $X_{P}$ and $X_{N}$ at the beginning of Phase $B$

$$
V_{o}=V_{t h p} \sqrt{\frac{8 \beta}{I_{o}}} \Delta V_{I N}
$$

where $V_{\text {thp }}$ is the threshold voltage of M5. The current $I_{0}$ is the average current through the drain of M11 during the set-up phase. The constant $\beta$ corresponds to the technology constant for an $\mathrm{n}$-type transistor given by the product $\mu_{\mathrm{n}} \mathrm{C}_{\text {ox }}$ for $\mathrm{M}^{25}$ where $\mu_{n}$ is the electron mobility in $n$-type silicon. The effective transconductance of the feedback latch $g_{m, e f}$ is given by

$$
g_{m, e f f}=g_{m, M 3}+g_{m, M 5}-1 /\left(r_{D S, M 3} \| r_{D S, M 5}\right)
$$

The terms $g_{m, M 3}$ and $g_{m, M 5}$ represent the transconductances of one inverter on one side of the feedback latch. A higher $g_{m}$ for those devices corresponds to an increased slew rate, reducing the time spent in the latching phase. The effective transconductance is lowered by the parallel output impedance of M3 and M5. Substituting Eq. 4.5, Eq. 4.6 and Eq. 4.7 into the expression for the total delay in Eq. 4.4 we obtain

$$
t_{d}=\frac{2 C_{o} V_{t h p}}{I_{o}}+\frac{C_{o}}{g_{m, e f f}} \ln \left(\frac{\Delta V_{o u t} \sqrt{I_{o}}}{\sqrt{2 \beta} V_{t h p} \Delta V_{I N}}\right) .
$$

The total delay $t_{d}$ in Eq. 4.8 is affected by the load capacitance which fluctuates with PVT variations ${ }^{26}$. Eq. 4.8 does not take this into account instead assuming equal capacitance on either side of the output nodes. To avoid a transition time based on unpredictable load capacitances ${ }^{27}$, the nodes $O_{N}$ and $O_{P}$ are buffered to present a

\footnotetext{
${ }^{25} \mathrm{C}_{\text {ox }}$ is the capacitance per area between the gate and the channel.

${ }^{26}$ During processing device length and width are not matched as well as doping concentrations.

${ }^{27}$ Unpredictable because the output of the comparator represent the asynchronous CLK signals for comparators in the next stage, the length of interconnect can vary greatly.
} 
more uniform impedance ${ }^{28}$.

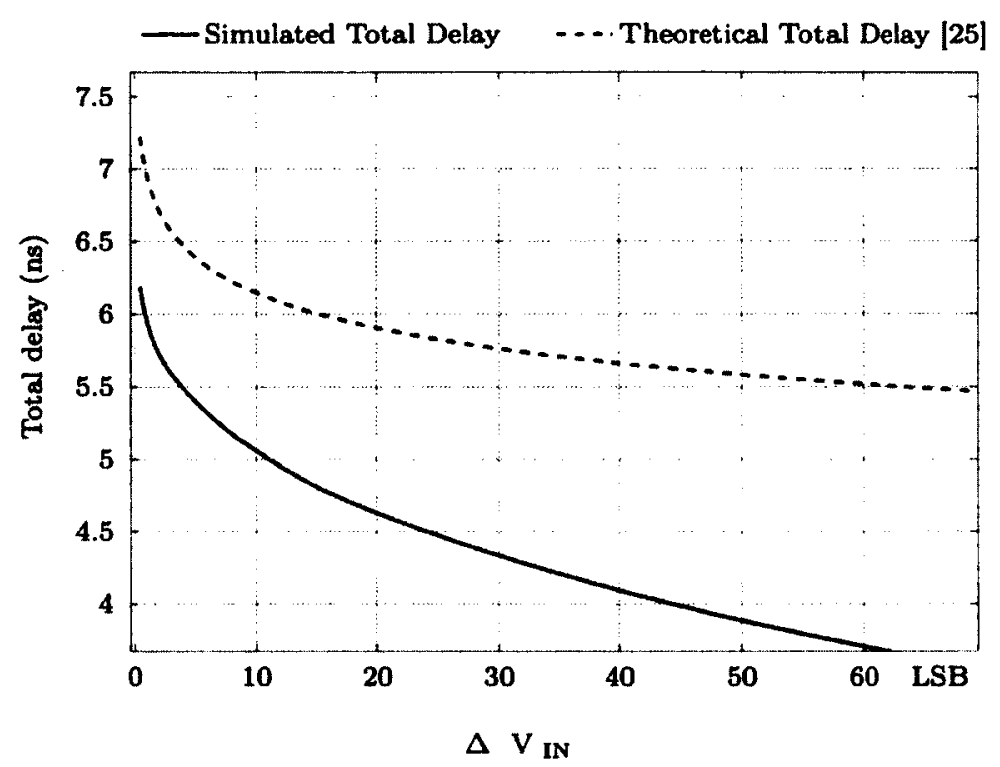

Figure 4.4: Simulation and theoretical total delay for a nominal comparator $\mathrm{V}_{\mathrm{os}}=0 \mathrm{mV}$ as a function of input voltage, theoretical curve is based on Eq. 4.8 from [25], the circuit parameters are extracted from transient simulations at the beginning of Phase B

The previous analysis can be reversed to examine the effects of an asymmetric variation in the time delay of Phase A or Phase B for the comparator. Examining Eq. 4.8, the value of $\Delta V_{\text {IN }}$ will change in order to compensate for the non-symmetric delay. Therefore a non-symmetric time delay can be transformed into an output referred input offset voltage. The method of implementing such an asymmetric time delay is to change to load capacitance on the positive or negative side, of one or both the nodes $\mathrm{X}_{\mathrm{P}, \mathrm{N}}$ or $\mathrm{O}_{\mathrm{P}, \mathrm{N}}$. This effect which is the basis for threshold adjustment is discussed in Sec. 4.1.3.

From Eq. 4.8 it is ascertainable that only the latching time of the feedback latch depends on the scale of the input voltage. As Phase B begins, the feedback latch

\footnotetext{
${ }^{28}$ Recall, the positive and negative outputs of the comparator serve as the asynchronous clock sigmal for comparators in the next stage. The distance can then vary from tens of $\mu \mathrm{m}$ to $\mathrm{mm}$ long lengths of interconnect.
} 
enters a metastable state for low differential inputs. In turn it takes longer for positive feedback to build and drive the latch to its final state. In Fig. 4.4 simulation results are presented for the total delay as a function of the differential input voltage $\Delta V_{\text {IN }}$ in terms of $1 \mathrm{LSB}$ of the ADC. The circuit parameters in Eq. 4.8 are extracted at the beginning of Phase B. Please note that the model in [25] was extracted from transistors with $\mathrm{W}=1 \mu \mathrm{m}$ and $\mathrm{L}=300 \mathrm{~nm}$ and therefore there is no guarantee of their applicability to the small channel devices used in the design of this comparator.

Although Eq. 4.8 models the trend of the comparator delay, there is an evident discrepancy between the simulated and theoretical results; it is therefore an unreliable estimator for extracting device sizes. The real merits of Eq. 4.8 is in providing an intuitive understanding of the greatest sources contributing to the total delay.

In the CABS ADC the highest frequency of operation is then determined by the total delay time through the lowest comparison value path ${ }^{29}$ and the highest comparison value path ${ }^{30}$. These two paths are presented in Fig. 4.5. The highest capacitance path corresponds to the largest imbalance in differential input pair widths used to achieve a desired threshold. As a consequence one of the regeneration nodes is loaded by a larger capacitance resulting in a longer comparison time. This approach to creating built-in references for the comparators is discussed in the next section. Through the lowest input voltage paths corresponding to codes, "0111111" to " 1000000 ", representing the MSB transition from 127 to 128 , comparators suffer from an increase in the duration of the metastable region in the comparison phase which in turn increases the total delay through the branch. As a quick reference Chap. 3 provides an overview of the entire CABS architecture, discussing in greater depth the binary decision paths through the ADC structure.

\footnotetext{
${ }^{29} \mathrm{~A}$ direct result of the duration of the meta-stable latching period being larger for smaller differential input voltages. Since the input is differential, the smallest comparison paths are those closes to VDD/2 for $1 \mathrm{LSB}$ representing codes 126 and 127.

${ }^{30}$ As a consequence of the large capacitance on one side of the output nodes.
} 
Both the empirical and theoretical approximations for $t_{d}$ yield valuable information in regards to design choices. Larger devices for the input differential pair can source more current, have a higher transconductance, resulting in a larger slew rate and reducing $t_{d}$. Increasing the latch feedback inverters, reduces the meta-stable duration of the latch phase at the expense of increasing the load capacitance at the drain of the input differential pair. There is an optimum point for any input differential pair for devices in the feedback latch yielding the lowest possible $t_{d}$. Larger devices lower the tuning range of threshold adjustment circuit unless they are scaled up as well. Scaling up the tuning devices removes the benefit achieved by larger devices by adding extra capacitance on output nodes. Therefore the tuning arrays and the comparator must be designed in unison. Porting the design to lower technology nodes, such as $90 \mathrm{~nm}$ or $65 \mathrm{~nm}$ offers a decrease in the total delay as the gate lengths of the devices shrink. The most critical observation is the effect of the differential input voltage on the total delay $t_{d}$. The worst case search paths through the ADC must be used as the benchmark when targeting a certain frequency of operation, as the delay through the cumulative stages determines the maximum frequency of operation. 


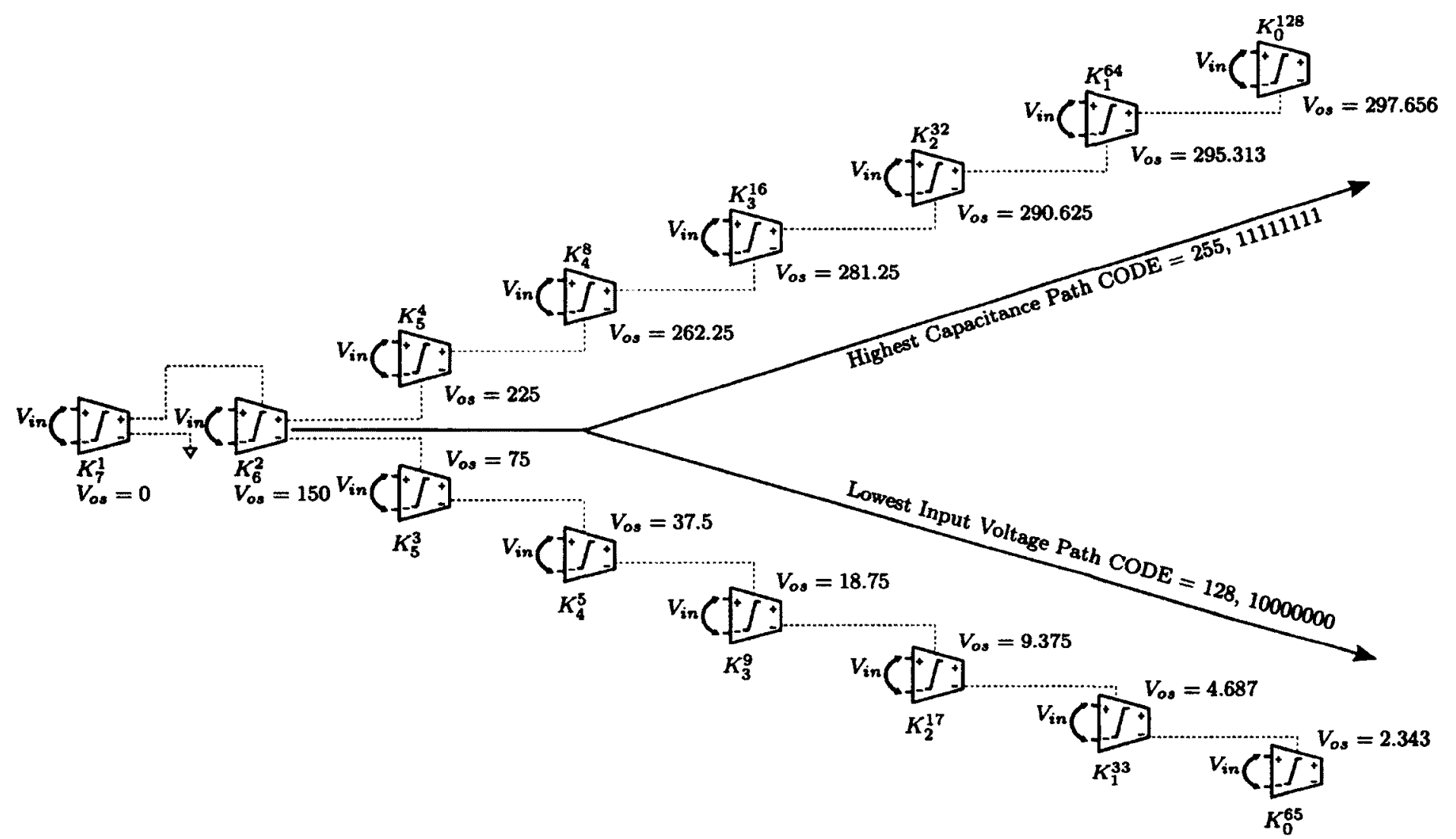

2
0
0
0
0
0
0
0
0
2
0
0
0
0
0
0
0
0

Figure 4.5: Highest capacitance and lowest input voltage binary search paths, the offsets $V_{o s}$ are the differential offsets expressed in $\mathrm{mV}$, away from $\mathrm{VDD} / 2$. 


\subsubsection{Offset and Threshold Configuration}

The comparator used in this design is a current steered, latch sense-amplifier. At its core lies a differential pair used to drive the currents through symmetric branches into the feedback latch. These devices in turn can be mismatched, as a consequence of random device-to-device variation (device mismatch) from implantation or systematic shifts from; wafer-to-wafer variation, poor layout techniques and local wafer variations [28]. These variations affect device parameter such as the threshold voltage $V_{t}$ and the current factor $\beta$ increasing the variance $\sigma_{I_{d}}^{2}$ of the drain to source current. This is translated into a different slew rate through the nodes $X_{N}$ and $X_{P}$ shifting the threshold of the comparator and appearing as an offset to the threshold of the comparator. A large number of analyses exist in publications dealing with mismatch variation [29-31] but quickly become cumbersome as more short-channel device effects are taken into account. The following relationships can be observed between the standard deviation of the threshold voltage, and the standard deviation of the relative current factor and the device sizes:

$$
\begin{gathered}
\sigma\left(\Delta V_{T}\right) \propto \frac{1}{\sqrt{W \cdot L}} \\
\left(\frac{\sigma(\Delta \beta)}{\beta}\right) \propto \frac{1}{\sqrt{W \cdot L}}
\end{gathered}
$$

Although it is tempting to assume that increasing the width of the devices is the best solution to reduce the mismatch of a parameter, better results can be obtained by decreasing the aspect ratio ${ }^{31}$ [32]. Naturally this depends on the circuit application, current mirrors benefit from a low aspect ratio (big $\mathrm{L}$ small $\mathrm{W}$ ) while the opposite is

\footnotetext{
${ }^{31}$ Using non minimum lengths for the devices.
} 
to be said for differential pair ${ }^{1}$ which lose accuracy as the slew rate decreases.

Increasing the aspect ratio of input differential pair leads to an decrease in the comparison time as the transconductance of the devices increases. In the interest of maintaining a higher frequency of operation, devices lengths were set to minimum lengths allowable by processing constraints at the expense of offset variance. Monte Carlo simulations which are based on empirical statistical models were used to examine the effect of device mismatch on the input offset. The result of a Monte Carlo simulation with both process and mismatch variation for the nominal comparators $^{32}$ is presented in Fig. 4.6. As an initial foray into the effects of mismatch on the comparator offset, a safety of margin of $1 \sigma_{V_{o s}}$ is used. In the following discussion the offsets and the standard deviation are normalized in terms of LSB where $1 \mathrm{LSB}=600 \mathrm{mV} / 255^{33}$.

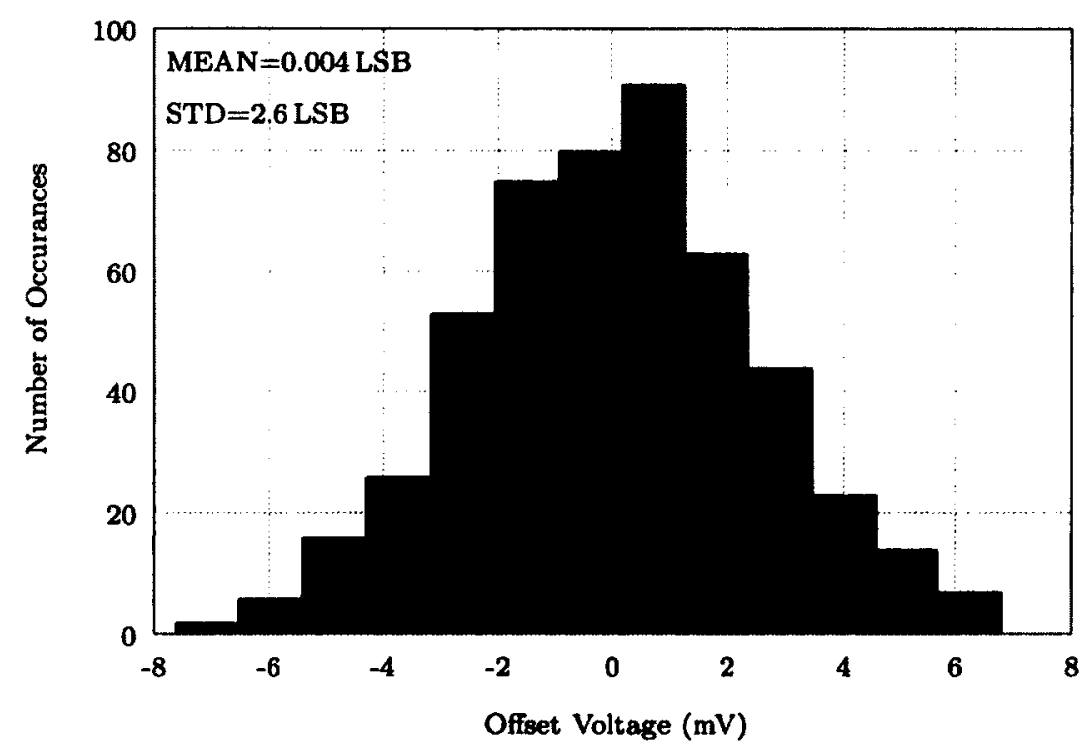

Figure 4.6: Monte carlo simulation for nominal comparator with $0 \mathrm{mV}$ threshold; $\mathrm{N}=600$, process and mismatch variation.

\footnotetext{
${ }^{1}$ Offset as a consequence of mismatch and the accuracy of a comparator are different, although the standard deviation of the offset may decrease, the accuracy of the comparator can degrade as the slew rate decreases and power consumption lowers [12].

${ }^{32}$ The nominal comparator has an offset $\mathrm{V}_{\mathrm{os}}=0 \mathrm{mV}$.

${ }^{33}$ From the familiar equation Eq. 4.1.
} 
For the nominal comparator, the standard deviation of the offset is $2.6 \mathrm{LSB}$ with a mean of $0.004 \mathrm{LSB}$. The value of $1 \mathrm{LSB}$ in the $8^{\text {th }}$ stage of the ADC is $2.353 \mathrm{mV}$. The maximum accuracy of the simulation itself is $0.085 \mathrm{LSB}$. Using a benchmark of only $1 \sigma \mathrm{V}_{o s}$ the offset of a single comparator is within the correct range $40 \%$ of the time. For design purposes a tolerance of $1 \sigma_{\mathrm{V}}$ or for the offset is unsatisfactory as it covers only $67 \%$ of the yield. To improve the success of the IC during fabrication, a benchmark of $3 \sigma \mathrm{V}_{\mathrm{os}}$ was adopted resulting in yield of only $13 \%$.

Although $3 \sigma_{\mathrm{V} \text { os }}$ is enough to guarantee the behavior of the offset of a single comparator within reasonable limits it does not guarantee the performance of the overall $\mathrm{ADC}$. The ADC requires a total of 255 comparators, given the interval of $3 \sigma \mathrm{V}_{\text {os }}$ at least one comparator can have an offset outside of the design specification. The problem is further complicated by the fact that the ADC has cascaded stages and multiple paths. To guarantee the INL and DNL specifications discussed further on in Chap. 5 , all the comparators through any given delay path must be within 1 LSB. The probability of having a correct threshold through any one of the 256 possible binary search paths is given by

$$
p\left(V_{o s}<L S B\right)=\prod_{1}^{8}\left(1-p^{n}\right)
$$

where "p", the probability of having $\mathrm{V}_{o s}<1 \mathrm{LSB}$ is given by the confidence interval corresponding to design goal of $\mathrm{k} \sigma_{\mathrm{V}_{o a}}$. For $\mathrm{k}=3$ as discussed above the width of the confidence interval is $99.9730 \%$. Using the probability of any one path having all comparators within the desired offset is $90.723 \%$. The probability of all paths in the ADC simultaneously functioning while being able to tune within a $3 \sigma_{\mathrm{V}}$ interval for the offset, is then given by

$$
p=(1-0.90723)^{256}
$$


which is effectively 0 . Using a confidence interval of $5 \sigma_{\mathrm{Vas}}$ the probability of one path working become $99.99 \%^{34}$ with an overall ADC working probability of $99.473 \%$. From this crude analysis one element in the design equation becomes clear, the ADC must be able to correct random variations of the offset up to $5 \sigma_{\mathrm{V}_{o s}}$ in order to guarantee the performance of the entire system ${ }^{35}$.

Consequently, the resolution of the LSB stages must be increased by reducing the offset variation or canceling the offset. Offset cancelation techniques typically require the addition of an extra clock phase [33]. The offset is first sampled on a capacitance and then subtracted from the input during the comparison phase. In the cascaded CABS structure a cancelation phase would be unrealistic, as a proceeding comparator would have to generate two phases for the cancelation scheme, paralyzing the speed advantages gained from the cascade structure. This method increases the power consumption and halves the frequency of operation. In preference to an offset cancelation strategy, arrays of programable binary weighted MOSCAP capacitors are used to generate the desired threshold levels and neutralize the inherent input offset. Arrays of 7 binary weighted capacitors are connected to the output nodes, meanwhile an array of linearly scaled units sized capacitors are connected directly to the regeneration nodes. The details of this technique are discussed in section Sec. 4.1.4.

In Fig. 4.7 the results of monte carlo simulation for the standard deviation of the offset are performed on the nominal comparator with varying device sizes. Initially the width of the devices is scaled by the same amount while maintaining minimum device lengths. From sensitivity and mismatch analysis it was found, as expected, that mismatch in the input pair was the largest contributor to offset variation. In

\footnotetext{
${ }^{34}$ The actual probability is 0.999979361299 .

${ }^{35} \mathrm{~A}$ confidence interval of $4 \sigma \mathrm{V}_{\text {os }}$ could be considered sufficient as it gives an overall ADC probability of functioning of $91.195 \%$.
} 
a second approach the length of the input differential pair was scaled by the same factor as the width. In other words the aspect ratio was maintained. This yielded a significant improvement of $22 \%$ for the $2 \mu \mathrm{m}$ comparator but increases the total delay for sizes larger than $2 \mu \mathrm{m}$. The first approach is prohibitive beyond $2 \mu \mathrm{m}$ as the width of the required MOSCAP tuning devices scales by the same amount in order to achieve the same linear tuning range increasing the delay and lowering the operating frequency. In Fig. 4.8 the total delay is presented as function of the input differential pair width. Maintaining the same aspect ratio for M1 and M2 while scaling up devices lowers the offset but increases the delay rapidly past the optimum device size of $2 \mu \mathrm{m}$.

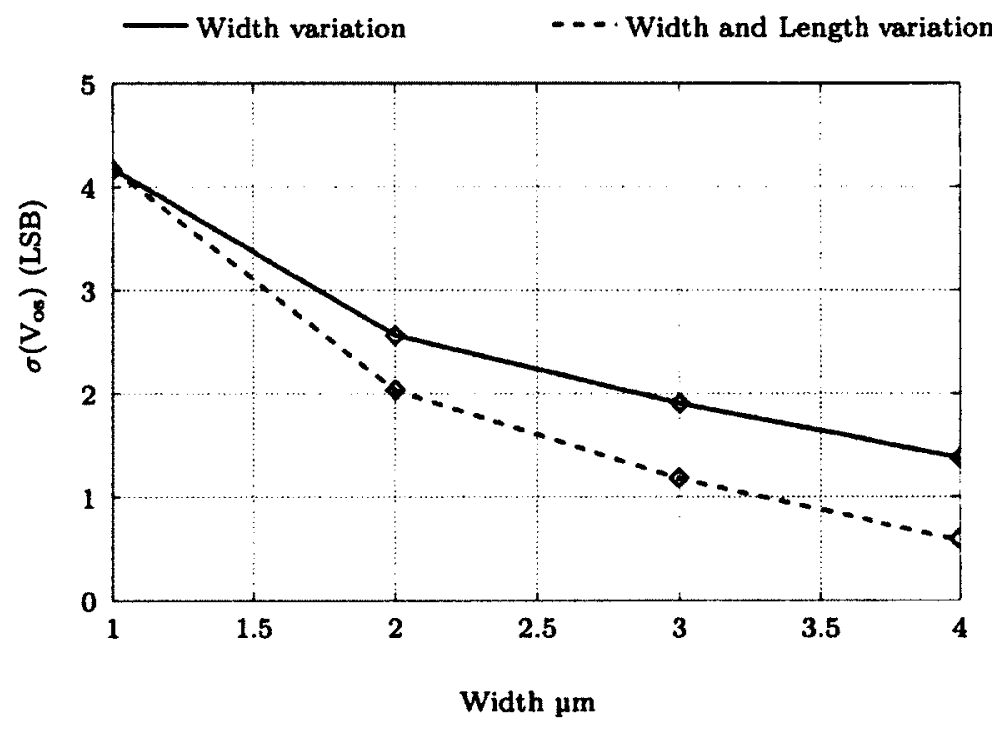

Figure 4.7: Standard deviation of the input offset as function of device width/length variation in LSBs, Monte Carlo; $\mathrm{N}=400, \mathrm{f}_{\mathrm{S}}=80 \mathrm{MHz}, \mathrm{V}_{\mathrm{IN}}=1 \mathrm{LSB}$, where " $\mathrm{N}$ " is the number of samples in the Monte Carlo simulation, $\mathrm{f}_{\mathrm{S}}$ is the sampling frequency with an ADC input voltage of $V_{\text {IN }}$. 


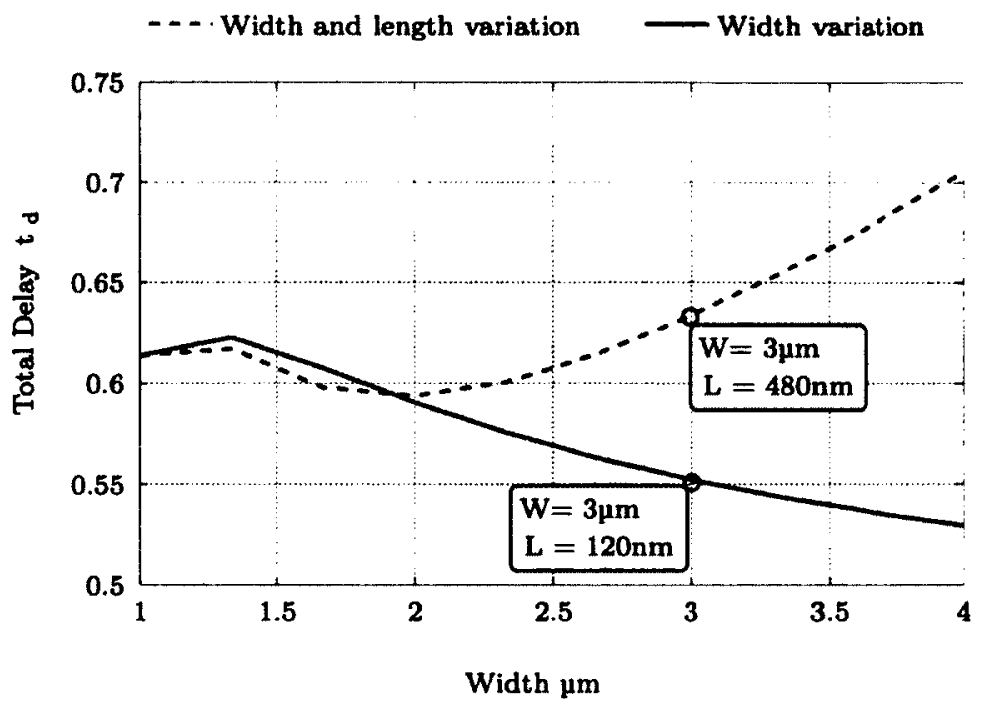

Figure 4.8: Total comparison delay as function of device width/length variation, Monte Carlo; $\mathrm{N}=400, \mathrm{f}_{\mathrm{S}}=80 \mathrm{MHz}, \mathrm{V}_{\mathrm{IN}}=1 \mathrm{LSB}$, where "N" is the number of samples in the Monte Carlo simulation, $\mathrm{f}_{\mathrm{S}}$ is the sampling frequency with an ADC input voltage of $V_{\text {IN }}$.

It is possible to take advantage of the concept of device mismatch by intentionally unbalancing the input differential pair and creating a predictable offset ${ }^{36}$ without the use of reference ladders or feedback mechanisms. An imbalance in the input devices is created such that the sizes of the input differential pair is given by

$$
W_{1}=\left(W+\frac{\Delta W}{2}\right) \text { and } W_{2}=\left(W-\frac{\Delta W}{2}\right) .
$$

To simplify the analysis, it is shown in [26], assuming that the square law model is valid during the first phase, "slewing" phase, of the comparator, the total input referred offset as a function of width imbalance is given by

$$
V_{i o} \approx \frac{\left(V_{G S}-V_{T p 1,2}\right)}{2}\left(\frac{\Delta W}{W}\right)-\frac{\left(V_{G S}-V_{T p 1,2}\right)}{32}\left(\frac{\Delta W}{W}\right)^{3} .
$$

\footnotetext{
${ }^{36}$ Note that the offset will still shift from PVT effects but will be centered around the desired, intentionally unbalanced offset. For the largest threshold the nominal offset is $275 \mathrm{mV}$ the offset will then vary by as much as $3 \cdot \sigma\left(\mathrm{V}_{\text {OS }}\right)$ around that value.
} 
In Eq. 4.14 the gate-to-source voltage $\mathrm{V}_{\mathrm{GS}}$ is given by

$$
V_{G S}=\left(V_{G S 1}+V_{G S 2}\right) / 2
$$

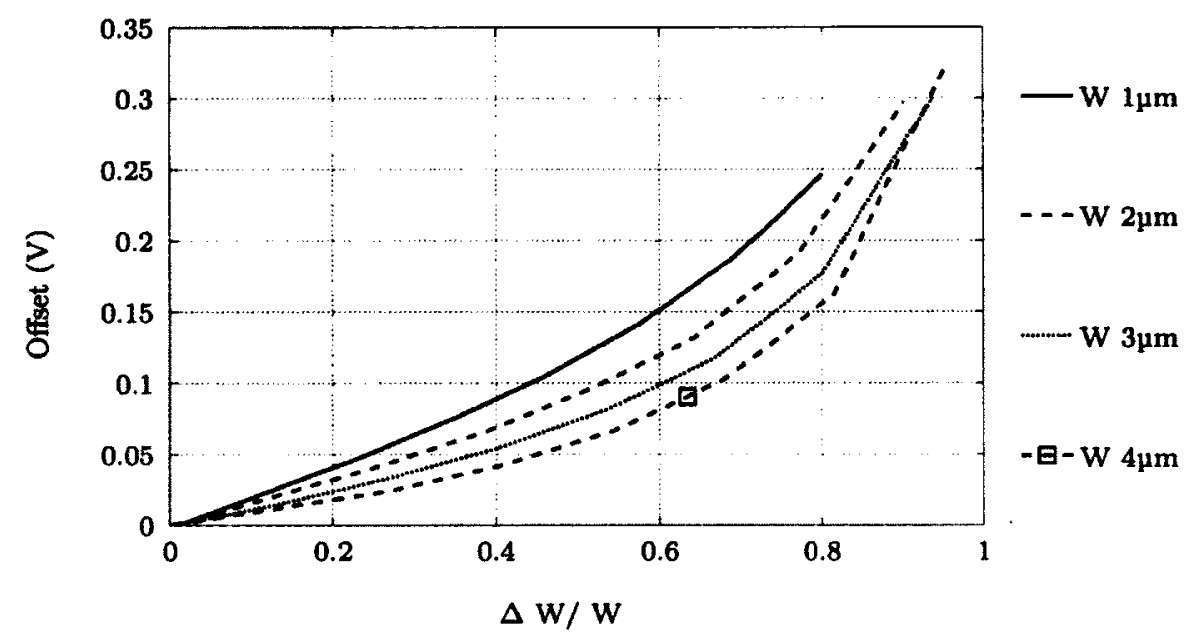

Figure 4.9: Offset as function of relative width imbalance $(\Delta \mathrm{W} / \mathrm{W})$ for different differential pair widths; $f_{S}=80 \mathrm{MHz}, V_{\text {iN }}=300 \mathrm{mV}$, where $f_{S}$ is the sampling frequency with an ADC input voltage of $V_{\text {IN }}$.

Sizing the input differential pair has the largest impact on the linear input range of the comparator. In Fig. 4.9, M1 and M2 are imbalanced according to Eq. 4.13 for device widths ranging from $1 \mu \mathrm{m}$ to $4 \mu \mathrm{m}$. Device lengths are kept to a minimum to increase the slew rate. Larger devices extend the linear range of the $A D C$, being able to produce higher threshold levels, at the expense of the offset scaling non-linearly in the upper threshold range. This implies that the effect of PVT on the offset will become more significant for larger $(\Delta \mathrm{W} / \mathrm{W})$. In turn the SCMTA tuning arrays must be designed to accommodate the required increase in tuning range for large imbalances ( $50 \%$ to $80 \%$ of the total width).

Using this method the first four stages of the CABS ADC were hard coded to 
their respective reference threshold value. Unlike [26] where the application is a low resolution 4-bit flash ADC, it would become difficult to generate the thresholds for the final four stages solely by employing device mismatch.

In the final four stages, intentional device mismatch is unified with the tuning mechanism presented in Sec. 4.1.4 to achieve the desired threshold offset and neutralize the effect of unintentional offset variation around the desired reference threshold. The resolution which can be achieved by using device imbalances is limited by minimum device size rules in the IBM CMRF8SF design kit. This is a direct consequence of fabrication constraints which limit the minimum adjustable step for the gate length to $10 \mathrm{~nm}$ increments on top of the minimum gate length of $120 \mathrm{~nm}^{37}$.

\subsubsection{Offset Tuning}

The final four stages of the CABS ADC use a combination of intentional device imbalance and offset tuning to establish reference thresholds. Using intentional device width imbalance (IDWI) eight comparators are generated, from $25 \mathrm{mV}$ to $275 \mathrm{mV}$ with a step of $25 \mathrm{mV}$.

Tuning the offset is performed by changing the capacitive loading at the regeneration nodes, $X_{N}$ and $X_{P}$, and the output node, $O_{N}$ and $O_{P}$, respectively. The analysis in [23] is similar to the one performed in [26] for device imbalance. The comparator was designed in conjunction with the switched capacitance MOSFET tuning arrays. Although the design of the tuning arrays was performed concurrently with the comparator, the design approach used in creating the SCMTAs is presented in Sec. 4.3 with considerable depth.

\footnotetext{
${ }^{37}$ As a sidenote, with the availability of parasitic extraction tools, it is possible to tweak the capacitances on the nodes further by changing the shape and size of interconnect or vias.
} 


\subsubsection{Complete Comparator Schematic Devices Sizes}

The device sizes for the nominal $0 \mathrm{mV}$ comparator are presented in $\mathrm{Tb}$. 4.1. The schematic of the full comparator is presented in Fig. 4.10. The comparators in the tuned core, the last four stages (MSB-4 to LSB), are presented in Tb. 4.2. For the final tuned stages, any desired threshold is within $10.2 \mathrm{mV}$ or $4.4 \mathrm{LSB}$ from the closest comparator. The rest of the tuning is completed with the SCMTAs. The threshold difference between comparators is $2 \mathrm{LSB}$ of the proceeding stage. In the $8^{\text {th }}$ stage the difference between the reference threshold is $4.7 \mathrm{mV}$ since the ADC LSB is $2.35 \mathrm{mV}$.

Table 4.1: Device size specifications for the nominal comparator.

\begin{tabular}{cccc|cccc}
\hline Transistor & Width $\mathrm{nm}$ & Length $\mathrm{nm}$ & Fingers & Transistor & Width $\mathrm{nm}$ & Length $\mathrm{nm}$ & Fingers \\
\hline M1 & 2000 & 120 & 8 & M12 & 2000 & 120 & 1 \\
M2 & 2000 & 120 & 8 & M13 & 640 & 120 & 8 \\
M3 & 2000 & 120 & 2 & M14 & 640 & 120 & 2 \\
M4 & 2000 & 120 & 2 & M15 & 320 & 120 & 2 \\
M5 & 1000 & 120 & 1 & M16 & 320 & 120 & 1 \\
M6 & 1000 & 120 & 1 & M17 & 640 & 120 & 1 \\
M7 & 1000 & 120 & 1 & M18 & 640 & 120 & 1 \\
M8 & 1000 & 120 & 1 & M19 & 320 & 120 & 1 \\
M9 & 500 & 120 & 1 & M20 & 320 & 120 & 1 \\
M10 & 500 & 120 & 1 & M21 & 1000 & 120 & 1 \\
M11 & 4000 & 120 & 6 & M22 & 320 & 120 & 1 \\
\hline
\end{tabular}

The choice was made to have gradated pre-defined sizes of comparators to avoid creating a large IDWI comparator library. It would have been possible to generate 180 different combinations (10 $\mathrm{nm}$ variation on the widths), closer to the desired reference than the current strategy employed. To simplify the relationship between the tuning codes and the desired threshold the choice was made to perform the most critical portion of the tuning using the SCMTA arrays. 
Table 4.2: Device size specifications for the input pairs of the comparators in the tuned core.

\begin{tabular}{ccccc}
\hline Offset $(\mathrm{mV})$ & $\mathbf{M}_{1}$ Width $(\mathrm{nm})$ & Fingers & $\mathbf{M}_{\mathbf{2}}$ Width $(\mathrm{nm})$ & Fingers \\
\hline 25 & 1720 & 4 & 2300 & 5 \\
50 & 1500 & 3 & 2500 & 5 \\
75 & 1290 & 3 & 2750 & 5 \\
100 & 1020 & 2 & 3000 & 2 \\
125 & 880 & 2 & 3120 & 6 \\
150 & 740 & 2 & 3360 & 6 \\
175 & 620 & 2 & 3420 & 6 \\
200 & 490 & 1 & 3570 & 7 \\
225 & 390 & 1 & 3640 & 7 \\
250 & 320 & 1 & 3710 & 7 \\
275 & 290 & 1 & 3850 & 7 \\
\hline
\end{tabular}

For the hard-coded core device sizes were custom tailored to the desired threshold and are presented in $\mathrm{Tb} .4 .3^{38}$

Table 4.3: Device size specifications for the input pairs of the comparators in the hard-coded core.

\begin{tabular}{cccccc}
\hline Bit & Offset & $\mathbf{M}_{1}$ Width $\mathbf{n m}$ & Fingers & $\mathbf{M}_{2}$ Width $\mathbf{n m}$ & Fingers \\
\hline B7 & 0 & 2000 & 8 & 2000 & 8 \\
\hline B6 & 150 & 740 & 2 & 3360 & 6 \\
\hline B5 & 75 & 1290 & 3 & 2750 & 5 \\
& 225 & 390 & 1 & 3640 & 7 \\
\hline B4 & 262.5 & 1020 & 2 & 3000 & 2 \\
& 187.5 & 880 & 2 & 3120 & 6 \\
& 112.5 & 740 & 2 & 3360 & 6 \\
& 37.5 & 620 & 2 & 3420 & 6 \\
\hline
\end{tabular}

\footnotetext{
${ }^{38}$ Where necessary parasitic layout contributions were used to an advantage in generating the precise threshold. Traces were made slightly wider to fractions of $\mathrm{fF}$, adjusting the capacitance at the regeneration nodes. This was done because the loading on the regeneration nodes is different in the HCC as opposed to the TC.
} 


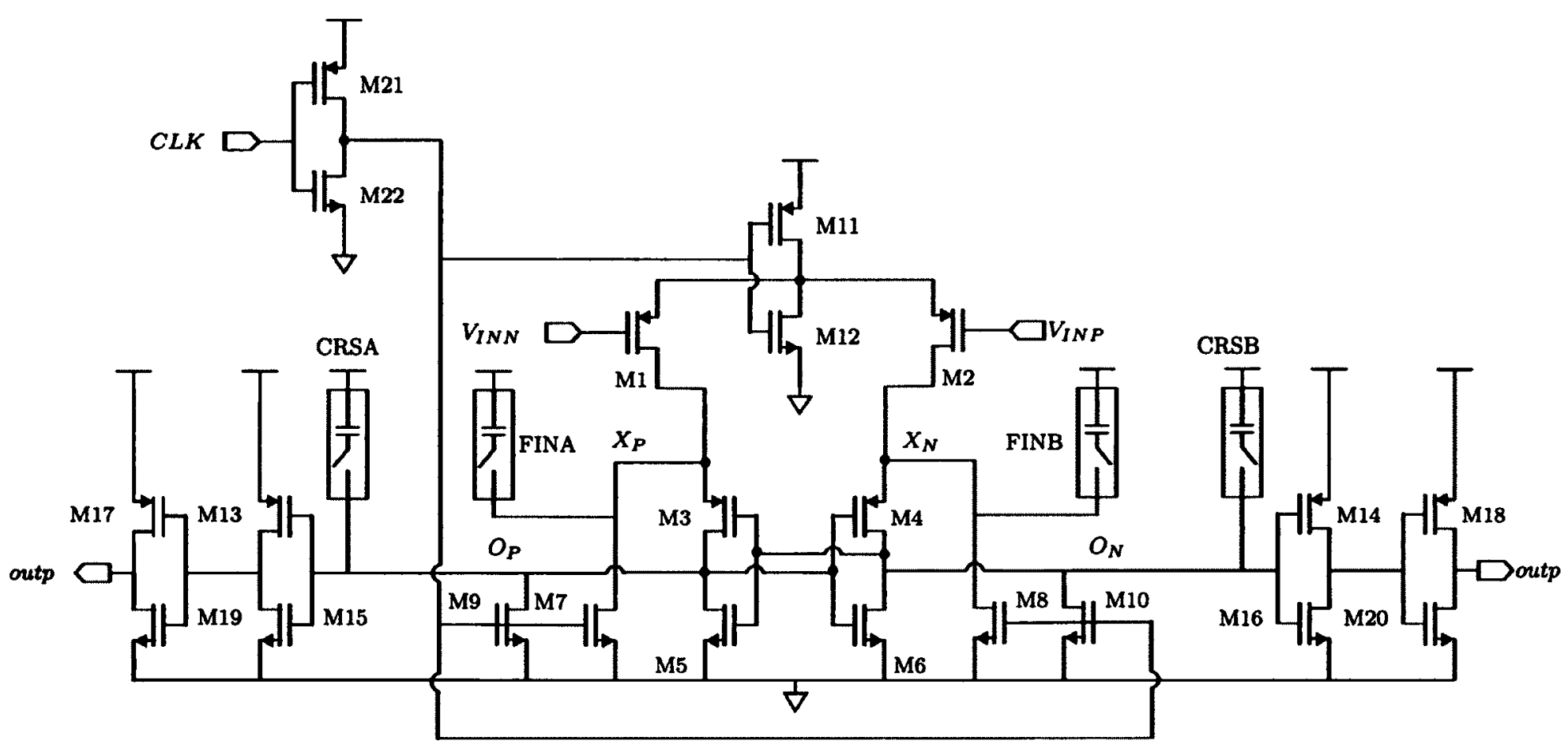

Figure 4.10: Full comparator schematic including tuning arrays. Although shown together, in layout the tuned arrays are implemented as a separate analog cell. The bitline driver (not pictured here) is also treated as a separate analog cell. 


\subsubsection{Comparator Layout}

The comparator layout went through several iterations. The initial comparator design presented in Fig. 4.11 made use of analog design techniques such as guard rings and dummy cells. Due to global device sizing constraints the design was dropped in favor of the design presented in Fig. 4.12. The initial comparator design is almost twice as high as the final design. Once the maximum design cell height was established, guard rings could no longer be used, being too close to the power rails and violating design rules. In Fig. 4.12 great care was taken to ensure a symmetric layout and maintain the same loading capacitance on the regeneration nodes and on the output nodes. The comparator layout illustrated in Fig. 4.12 also includes the bitline drivers presented in Sec. 4.2 .

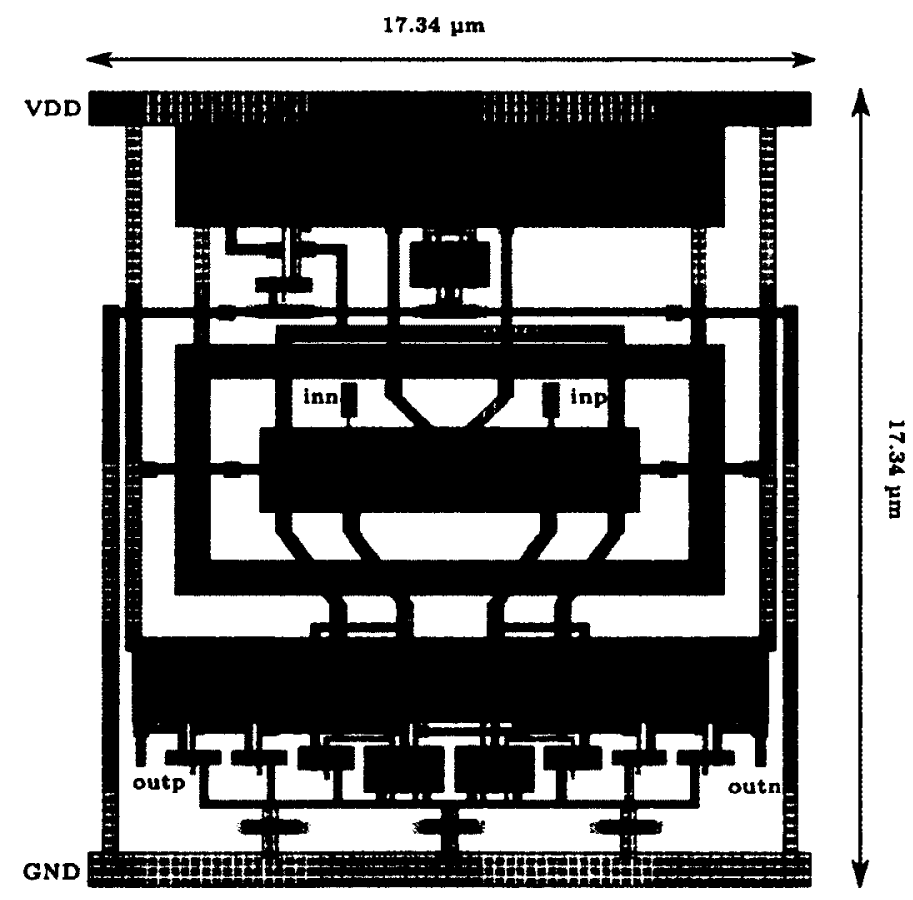

Figure 4.11: Initial comparator layout, $0 \mathrm{mV}$ threshold comparator. 


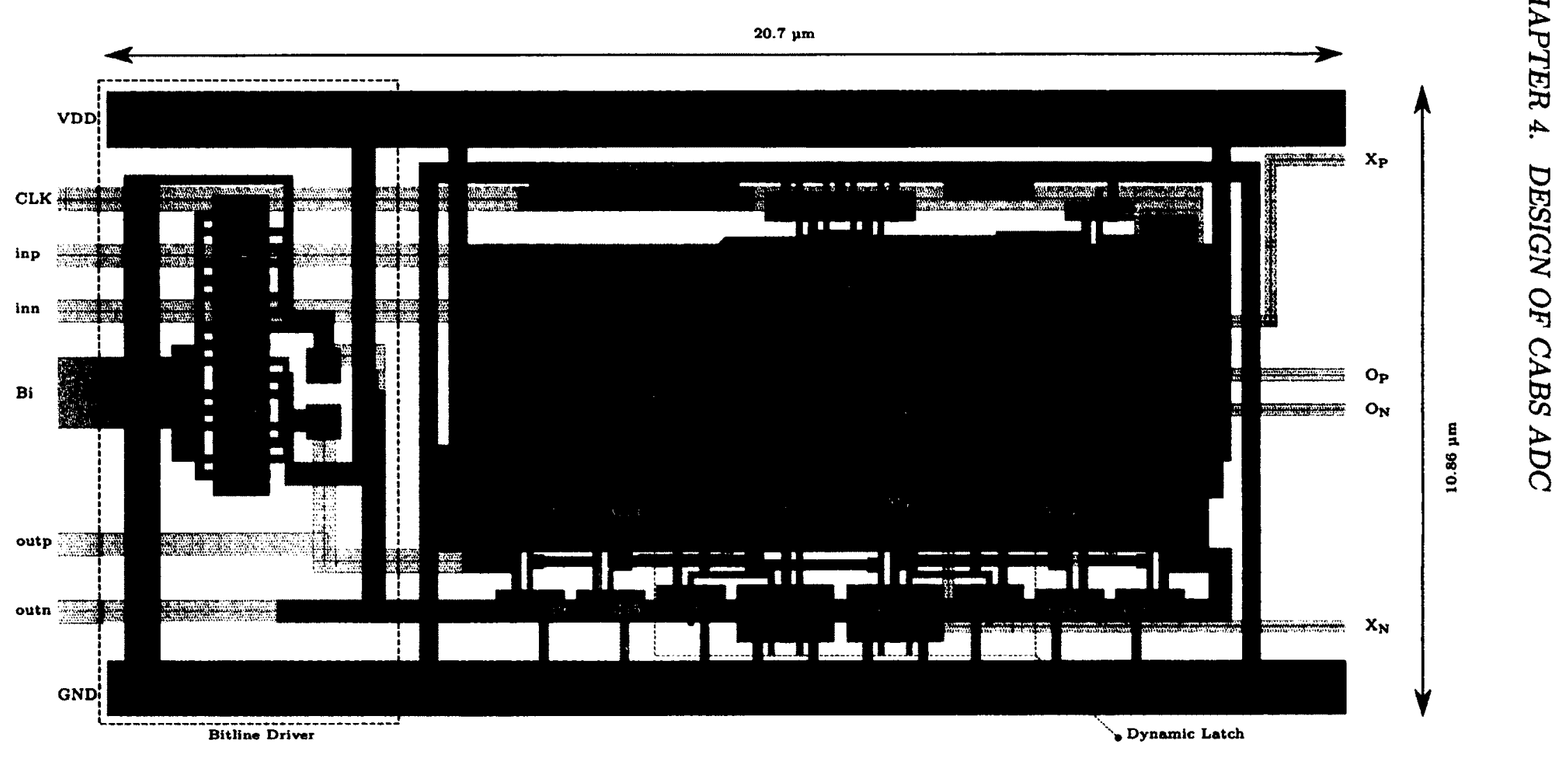

Figure 4.12: Final comparator layout, $0 \mathrm{mV}$ threshold comparator. 


\subsection{Bitline Drivers}

For any given stage the bit is determined by decision of one comparator ${ }^{39}$ as the decision propagates through a binary search path. A logical "1" decision corresponds to a value within the range above the given threshold, while the opposite is true for logical " 0 " decision. A bitline encoder/driver is used to set the correct bit value, and prevent direct loading between comparator output nodes in a stage. An example of a positive bitline driver is presented in Fig. 4.13.

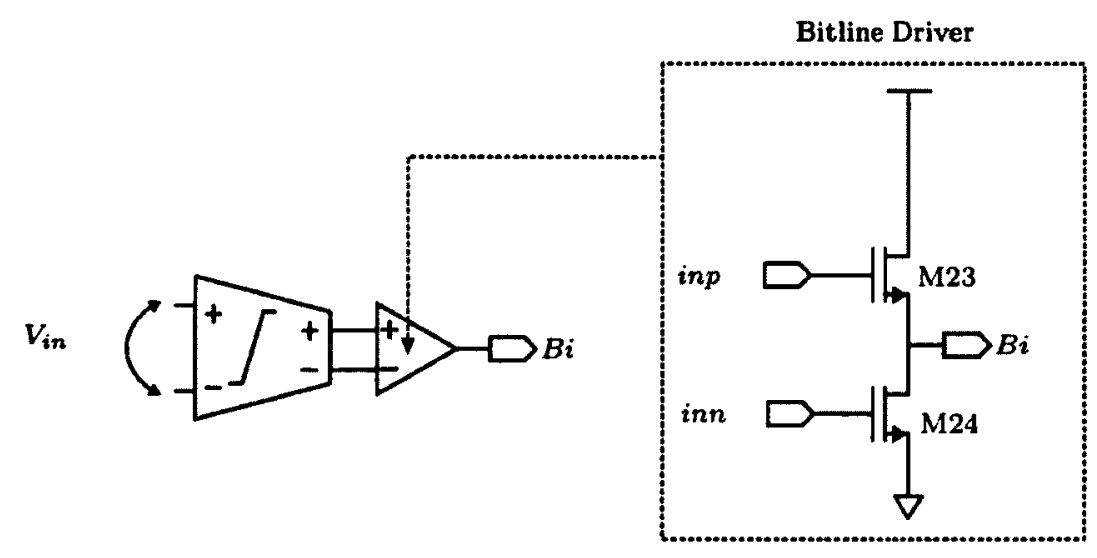

Figure 4.13: Positive threshold bitline driver.

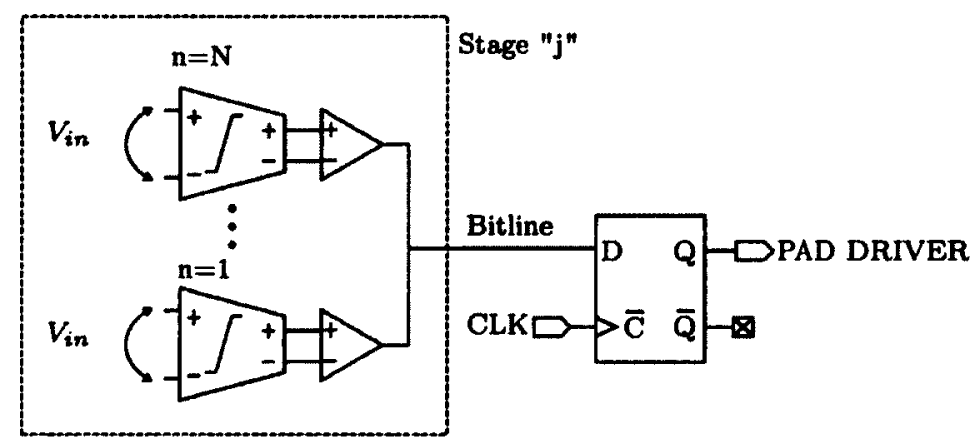

Figure 4.14: Comparators with connected bitline drivers and sampling falling edge D Flip-Flop, for example; stage $j=8$ determines the LSB bit, b0, with 128 comparators, the output of all bitline drivers is connected to the same bitline.

${ }^{39}$ Only one comparator is triggered in any given stage. 
The output of all "n" encoders in a stage must be tied together to one "bitline" as shown in Fig. 4.14. Since only one comparator is active at a time, only the parasitic capacitance $c_{j b}$ and $c_{d b}$ of the inactive drivers contribute to the bitline capacitance. In simulation the worst case bitline capacitance of $1 \mathrm{pF}^{40}$ is used to take into account the approximate capacitance contributed by the physical layout in addition to the parasitics from the transistors.

In Fig. 4.15 a transient simulation shows the behaviour of the bitline encoder along with the comparator and flip flop delays. The $t_{c b}$ delay, necessary to charge the bitline, becomes an issue in the final stage of the ADC where the comparison output approaches the falling edge of the clock and the cascaded output can "run outside" of the falling clock edge for higher clock frequencies. This causes the flip flop to sample the bitline incorrectly by sampling the logic "0" portion of the bitline transition resulting in a missing code. The shaded region in Fig. 4.15 corresponds to an invalid sampling region for the bitline.

When node "outp" is at a logic "1", M24 is in the cut-off region and M23 is in triode. The output node "Bi" approacheds $V_{D D}-I_{D} r_{D S}$ where $I_{D}$ is given by

$$
I_{D}=\mu_{n} C_{o x}\left(\frac{W}{L}\right)\left[\left(V_{G S}-V_{T n}\right) V_{D S}-\frac{V_{D S}{ }^{2}}{2}\right]
$$

and $r_{D S}$ is given by

$$
r_{d s}=\frac{1}{\mu_{n} C_{o x}\left(\frac{W}{L}\right)\left(V_{G S}-V_{T n}\right)} .
$$

If the "outn" is at logic " 1 " M23 enters cut-off and M24 pulls down the bitline to some value $I_{D} r_{D S}[34]$.

Adequately sizing the bitline driver is critical for ensuring the desired frequency of

\footnotetext{
${ }^{40}$ This capacitance would correspond to a $7.194 \mathrm{~mm}$ long transmission line for a minimum E1 metal width which is a large over estimation, no bitline on the IC is greater than $1.5 \mathrm{~mm}$.
} 


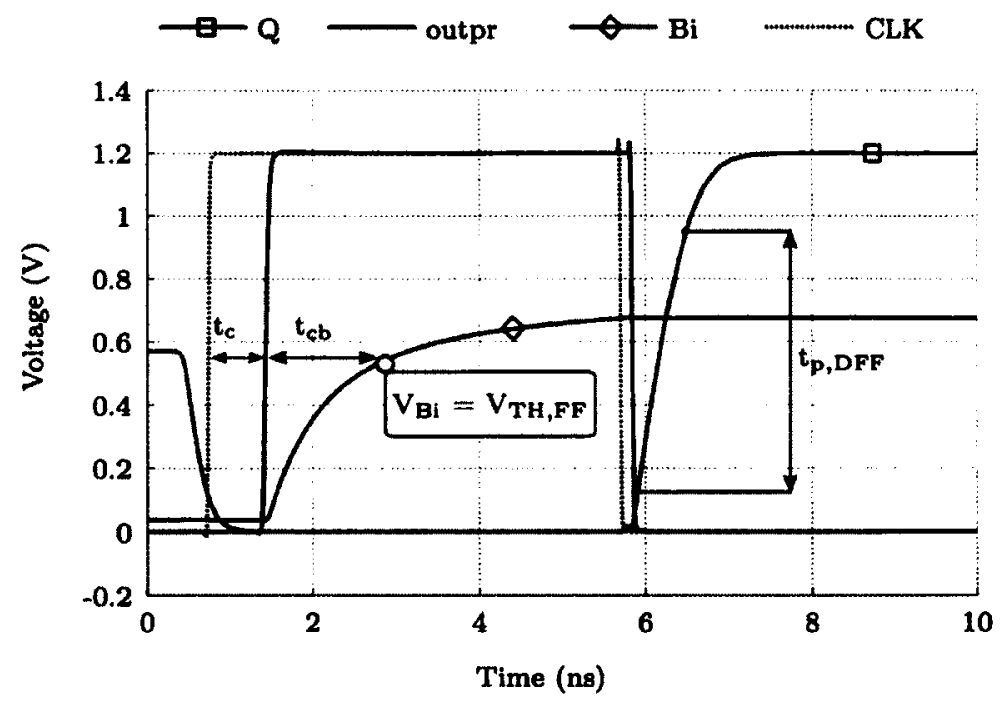

Figure 4.15: Transient simulation including bitline encoder and falling edge flip flop, $t_{c}$ is the comparison delay, $t_{c b}$ is the delay between $V_{D D} / 2$ of outp and the point where the bitline is charged to input threshold of the falling edge flip flop, $t_{p, D F F}$ is the propagation delay of the falling edge flip flop; $f_{S}=100 \mathrm{Mhz}, V_{I N}=$ $0.1 \mathrm{mV}$.

operation. In Fig. 4.16 (left) the propagation delay as percentage of half a duty cycle of the clock signal is plotted against the number of drivers in any given stage. The $8^{\text {th }}$ stage of the ADC, bit0, requires 128 bitline drivers connected in parallel. Although only 1 bitline driver is active at a time, all inactive encoders contribute some parasitic capacitance $\mathrm{C}_{\text {driver,off }}$. The trade off is made between power consumption and delay, as is typical for any buffer type "circuit". With increasing size, excessive noise can also be coupled into the substrate or into the power supply lines. 

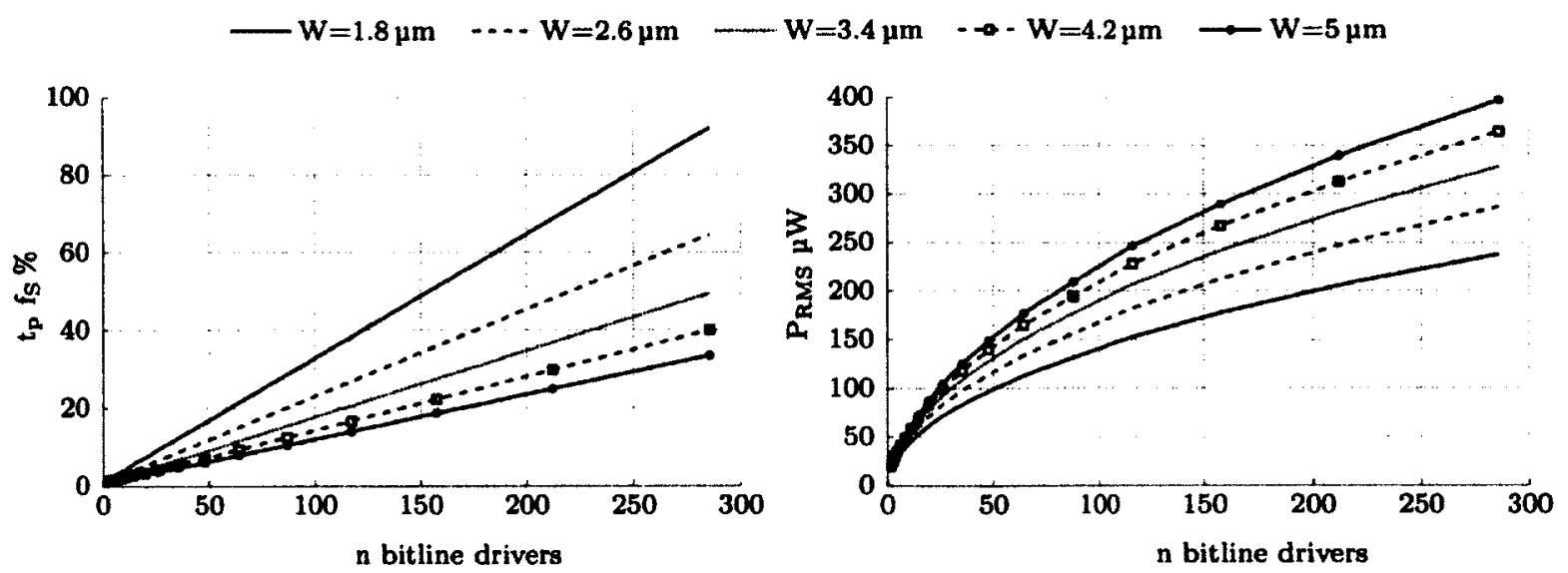

Figure 4.16: Propagation delay as a percentage of half the duty cycle of the CLK signal (pictured left), RMS power consumption (pictured right), both plotted as a function of the capacitive loading of $n$ bitline drivers (where $C_{\text {driver,off }}=$ $7 \mathrm{fF}$ for a $\mathrm{W}=4 \mathrm{um}$ device) for varying device widths. 


\subsection{Switched Capacitance MOSFET Tuning Array (SCMTA)}

\subsubsection{Method of Capacitive Imbalance}

As discussed in Sec. 4.1.4 tuning the comparator's threshold is carried out by creating an intentional capacitive imbalance on one side of the regeneration and output nodes. For a more complete presentation the reader is encouraged to examine [23], the presentation below is a summary of the work presented therein. Similar to [26], the linear region of operation is assumed. The output nodes are capacitively unbalanced resulting in a capacitance on each output node of

$$
C_{O 1}=\left(C_{O}+\frac{\Delta C_{O}}{2}\right) \text { and } C_{O 2}=\left(C_{O}-\frac{\Delta C_{O}}{2}\right)
$$

The notation from [23] has been maintained in this presentation. The node capacitance " $\mathrm{C}_{\mathrm{O}}$ " is the capacitance at the output node $\mathrm{O}_{\mathrm{P}}$ and " $\mathrm{C}_{\mathrm{O} 2}$ " is the capacitance at the output node $\mathrm{O}_{\mathrm{N}}$.

It is assumed that the devices are operating in the saturation region of Phase A and Phase B. The comparator input threshold is first order proportional to the effective gate to source voltage of transistors $M 1$ and $M 3^{41}$. This is the typical square law model based expression for the transconductance [26] given by

$$
g_{m}=2 \cdot I_{D} /\left|V_{G S 1}-V_{T p}\right|
$$

\footnotetext{
${ }^{41}$ Only one side of the dynamic latched comparator is examined.
} 
The input offset created through an intentional capacitive imbalance is given by

$$
\Delta V_{I N} \approx\left|\frac{V_{G S 1}-V_{T p 1}}{4}\right| \frac{\left|V_{G S 3}-V_{T p 3}\right|}{\left|V_{T p 3}\right|} \cdot\left(1+\frac{V_{D D}-V_{T i n u}}{V_{T n 5}}\right) \frac{\Delta C_{O}}{C_{O}},
$$

where $\mathrm{V}_{\text {Tinv }}$ is metastable voltage of the latch during Phase $\mathrm{C}$ which can be approximated as $V_{D D} / 2$ for a "non-skewed" cross coupled inverted pair where the PMOS transistor is scaled up to obtain a threshold voltage of $\mathrm{V}_{\mathrm{DD}} / 2$. The gate to source voltage of $M 1$ or $M 2, V_{G S 1}$, in Phase $A$ can be approximated as $V_{C M}-V_{D D} . V_{G S 3}$ is the average gate-to-source voltage of latch transistor M3 or M4 in Phase B which can be estimated by

$$
V_{G S 3} \approx V_{T n 5}+V_{T p 3}-V_{D D}
$$

The equation presented in Eq. 4.20 for the threshold shift is an extension of Fig. 4.14 but takes into account the effects of M3 and M5 on the generated offset. From this equation it can be asserted, for a fixed desired input offset, a bigger relative loading capacitance $^{42}$ can be seen at the nodes for lower over drive voltages corresponding to smaller device sizes for M1,M3,M5 for the same supply voltage. This behaviour is verified in Fig. 4.20.

\subsubsection{MOSCAP Varactor Arrays}

The capacitive imbalance $\Delta \mathrm{C}$ in Eq. 4.20 is established through PMOS varactor arrays. An array of binary weighted devices is connected to the output nodes, providing 7 bits of coarse tuning on each side. This coarse tuning array is pictured in Fig. 4.17. The nominal device size is $320 \mu \mathrm{m}$ by $0.12 \mu \mathrm{m}^{43}$. An array of linearly scaled devices

\footnotetext{
${ }^{42}$ Note that for smaller devices the capacitance contributed to the output and regeneration nodes decreases but the relative capacitance on the nodes increases.

${ }^{43}$ The nominal device size refers to $\mathrm{bO}$ of the tuning array, or the LSB of the tuning array not the global LSB.
} 
is connected to regeneration nodes $X_{P, N}$ to provide 4 additional fine tuning levels interpolating between the 2 LSB bits of the coarse array. The fine tuning array is presented in Fig. 4.18.

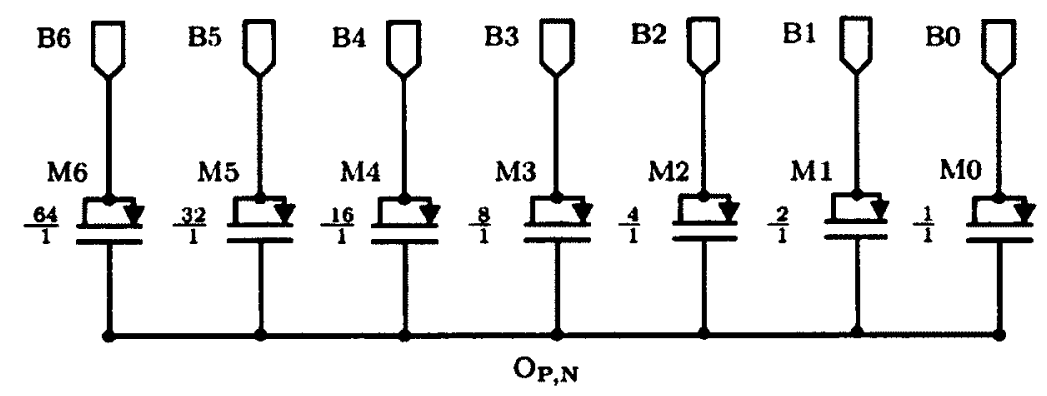

Figure 4.17: Coarse tuning array with 7 standard, binary scaled PMOS devices implementing 7 bits of tuning, the nominal device M0, is $320 \mu \mathrm{m}$ by $120 \mathrm{~nm}$

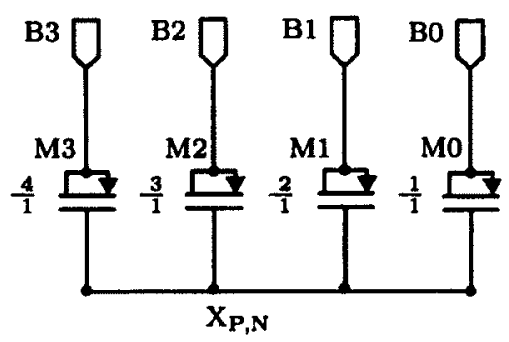

Figure 4.18: Fine tuning array with 4 standard, linearly scaled PMOS devices, the nominal device $\mathrm{M} 0$, is $320 \mu \mathrm{m}$ by $120 \mathrm{~nm}$

SCMTA devices suffer from device mismatch and variation. Mismatch will result in a different absolute threshold being generated on either side of the imbalance for the same tuning code, while variation resulting from processing (gate thickness, doping concentration, device skew) will randomly affect the capacitive contribution $\Delta \mathrm{C}$ of a particular tuning code for each tuning array. Devices within $150 \mu \mathrm{m}$ of each other will track closely [35]. Across the same chip, these variations will become significant and cause problems for the tuning algorithm as one tuning code will not have the same $\Delta \mathrm{C}$ for comparator's in different regions of the chip (several LSBs). 
For the MOSCAP capacitor, the only physical parameters which the designer has control over are the length and the width of the device. Some of the types of variation for those parameters are; site-to-site variation, horizontal-vertical, nestedto-isolated, total across-chip variation [35]. To reduce the standard deviation of the tuned threshold, the aspect ratio of the devices could be reduced e.g., $320 \mu \mathrm{m}$ by $320 \mathrm{~nm}$ for the $1 \mathrm{LSB}$ device (M0). The rest of the devices would have the same scaling as shown in Fig. 4.17.

The effect of $1 \mathrm{LSB}$ of tuning corresponding to b0 being active in Fig. 4.17, shifts the threshold by 0.18 LSB for a nominal threshold comparator with nominal sized pFET devices $(320 \mu \mathrm{m}$ by $120 \mathrm{~nm})$. Monte Carlo analyses were performed to determine the statistical parameters of interest and the effect of device and mismatch and process variation on the tuning arrays ${ }^{44}$. In Fig. 4.19 the results of such a Monte Carlo analysis for the tuned threshold (offset) of the comparator is shown as a function of the global LSB of the ADC.

Increasing the aspect ratio of the MOSCAPS lowers the variance of $\triangle \mathrm{C}$, however, the minimum tuning resolution begins to approach $0.5 \mathrm{LSB}$ and greater as the gate length is increased. The contribution to the capacitance is approximated by Eq. 4.22. Doubling the length, approximately doubles the additional delay contributed to the comparator $t_{c}$ delay, lowering the frequency of operation. This effect is intensified for full scale input paths where the capacitance is larger from intentional device width imbalance. The results of the discussion above can be seen in Fig. 4.20 where the comparison delay and the tuned threshold (relative to reference threshold) are plotted as a function of gate length. The minimum tuned threshold corresponds to the LSB M0 device being active in the coarse tuning array.

\footnotetext{
${ }^{44}$ Contributions to the offset as a consequence of device mismatch and PVT in the comparator were ignored.
} 


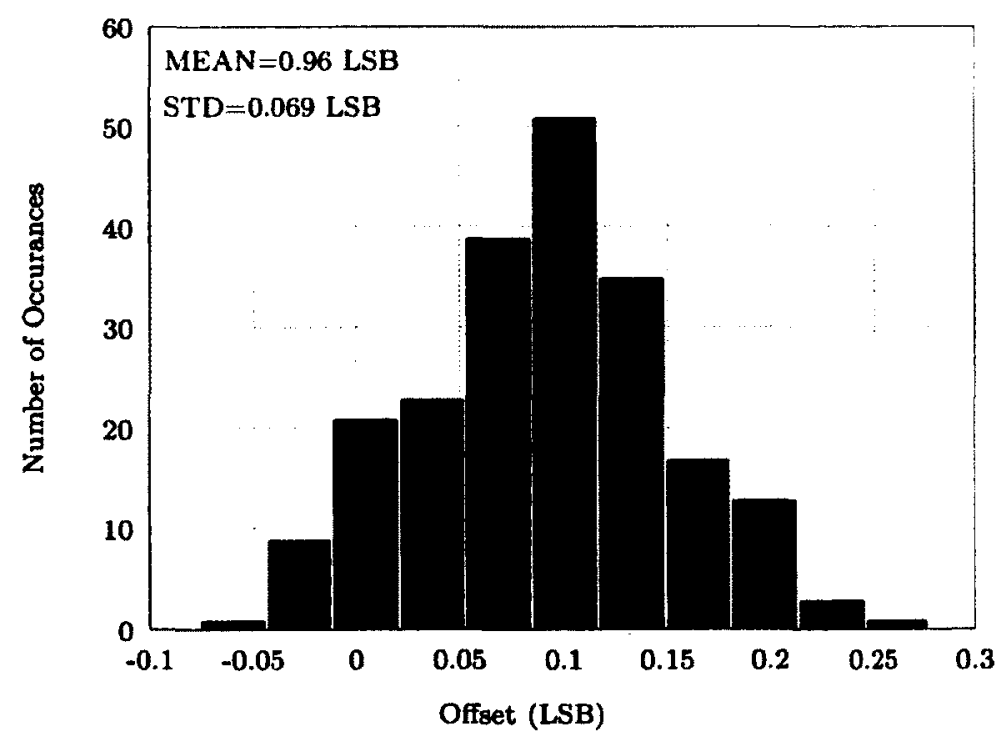

Figure 4.19: Monte carlo simulation for the offset in terms of ADC LSB for 1 LSB active in the coarse tuning array; $\mathrm{b} 0=1.2 \mathrm{~V}, \mathrm{~N}=200, \mathrm{f}_{\mathrm{S}}=80 \mathrm{MHz}, \mathrm{V}_{\mathrm{IN}}=1 \mathrm{LSB}$.

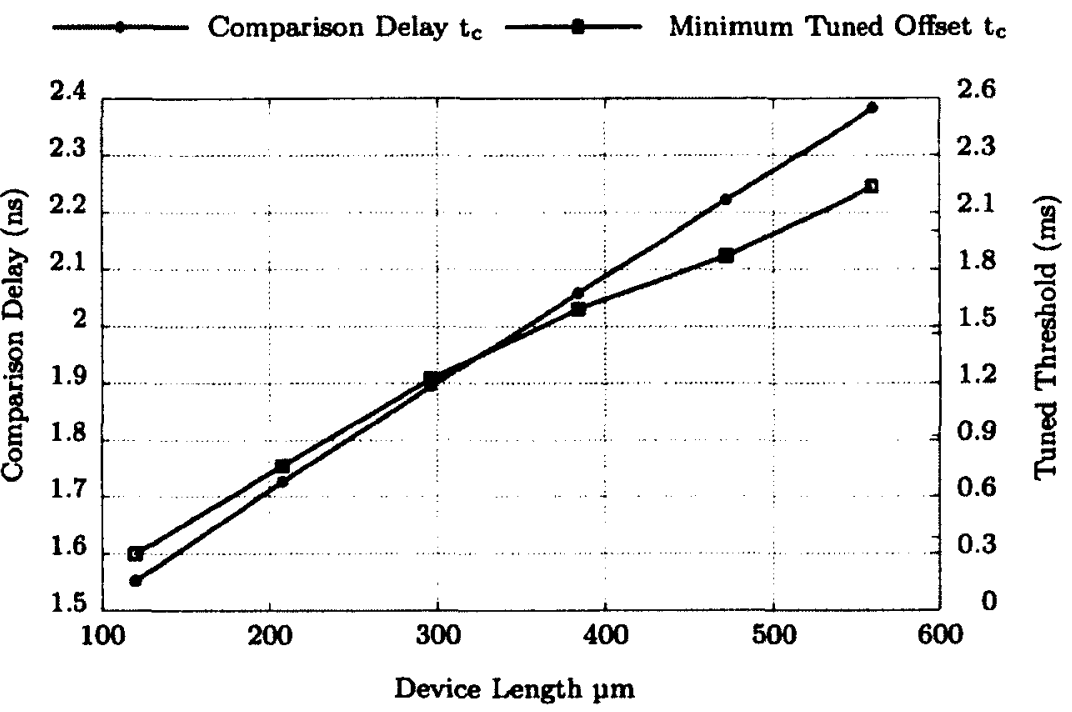

Figure 4.20: Comparison delay and the minimum tuned threshold as a function of device length; the minimum tuned threshold corresponds to CODE $=000001$, $\mathrm{M} 0$ active in the coarse tuning array, $\mathrm{f}_{\mathrm{S}}=80 \mathrm{MHz}, \mathrm{V}_{\mathrm{IN}}=1 \mathrm{LSB}$

The MOSCAPS have their source and drain tied together and connected to a control signal which is ideally "0" when disabled or "1.2" volts when the FET is operational. For the IBM CMRF8SF $0.13 \mu \mathrm{m}$ technology, gate leakage is higher for 
n-channel devices than p-channel devices [36], [35].

The gate of the device is connected to one of the comparator's output nodes. The control signal for the MOSCAP comes from a 22-bit parallel memory built from a shift register which is examined further in Sec. 4.4. During the slewing-phase and the regeneration phase the common, shorted source and drain voltage is $1.2 \mathrm{~V}$ and the gate voltage swings between $0 \mathrm{~V}$ and approximately $\mathrm{V}_{\mathrm{DD}} / 2$. Since it is desired to maintain a constant capacitance, regardless of the swing on the regeneration nodes, the device is operating deep in the accumulation region where there is no inversion layer formed under the gate. Given the established bias conditions, this region of operation is guaranteed for p-channel transistor.

When the device is disabled, it still contributes a capacitance to the output nodes mainly from the drain and source overlap regions. Since the capacitance is proportional to the width (length is maintained the same for all devices) the MSB, M6 device will contribute $64 \mathrm{X}$ the "off" capacitance of the M0 device. The capacitance as a function of the terminal voltages $\mathrm{V}_{\mathrm{SD}, \mathrm{B}}$ and $\mathrm{V}_{\mathrm{G}, \mathrm{B}}$ is illustrated as a contour plot in Fig. 4.21.

As the channel is relatively uniform between the source and drain, $c_{g s}$ and $c_{d s}$ capacitances add to yield

$$
\Delta C \approx C_{o x} W L,
$$

along with a contribution, albeit a small contribution, from the external gate capacitances $c_{\text {gse }}$ and $c_{g d e}$ which can be ignored for estimation purposes.

The extrinsic capacitances $c_{s b, e}$ and $c_{d b, e}$ are primarily comprised of the sourceto-bulk and drain-to-bulk reverse bias capacitances, overlap and fringe capacitances [37]. For this process the standard p-channel device sits in an n-well within a p-type substrate. The pfet then has the advantage of not directly loading the output nodes 


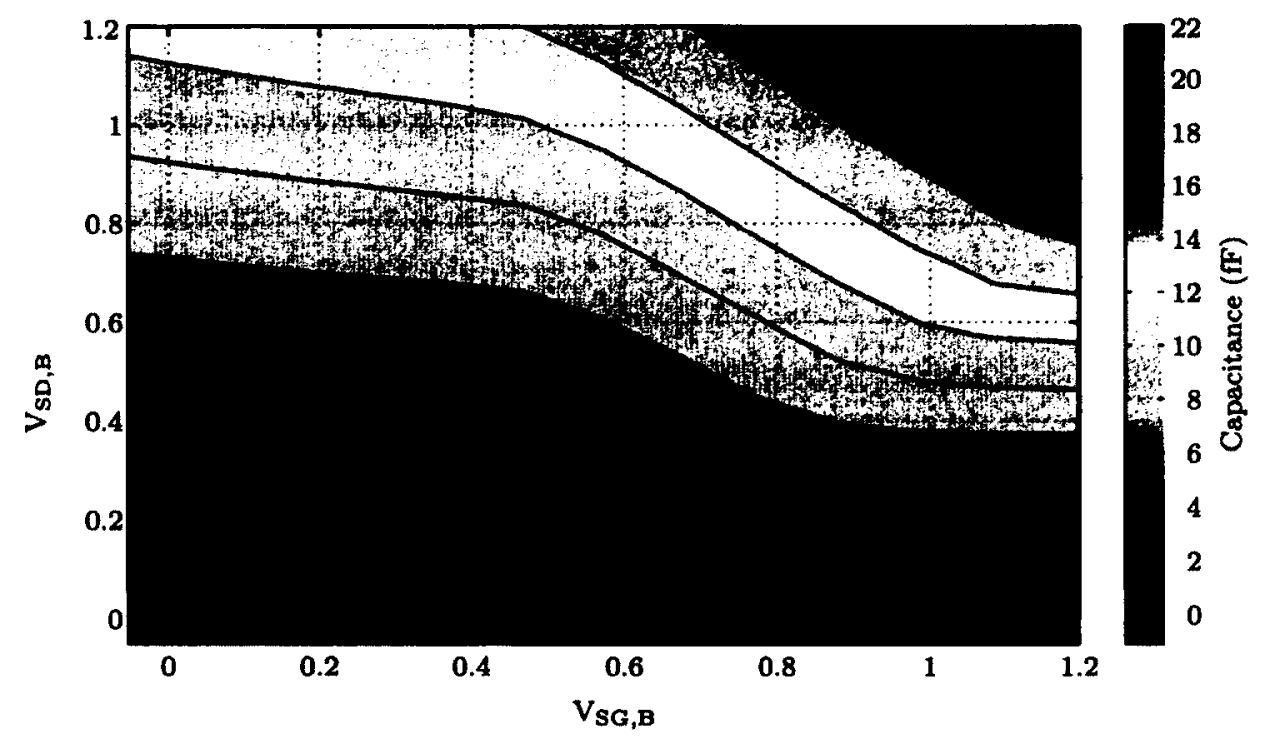

Figure 4.21: Contour plot of the capacitance of a MOSCAP (M6) device for different terminal potentials covering all regions of operation, the device is on when $V_{S D, B}$ $=" 1.2^{\prime \prime} \mathrm{V}, \mathrm{V}_{\mathrm{SG}, \mathrm{B}}$ swings between $0 \mathrm{~V}$ and $1.2 \mathrm{~V}$.

as a result of parasitic coupling through the extrinsic capacitances. Therefore the ratio of $I_{\text {on }}$ to $I_{o f f}[23]$ is higher for a p-channel device as opposed to an $n$-channel device yielding a larger $\Delta \mathrm{C}$ for the same layout area.

The comparator threshold is plotted as a function of the tuning code in Fig. 4.22. For a desired positive threshold shift, corresponding to a positive tuning code, the enabled coarse array (CRSB) in Fig. 4.10 becomes active, increasing the capacitance on the negative output node. The $3 \sigma$ of the offset corresponds to roughly $16 \mathrm{LSB}$ necessary to correct for process variations. The rest of the 12 LSB is used to tune the threshold up to a maximum of 6 LSB with a safety of margin. The fine tuning array contributes an additional 2 LSB to the tuning range.

The accuracy of the comparator must be lower than LSB/4 to the offset [33], allowing a safety of margin for the contribution from noise. Given the method used in obtaining the threshold in simulation (long input ramp) there is a finite precision 


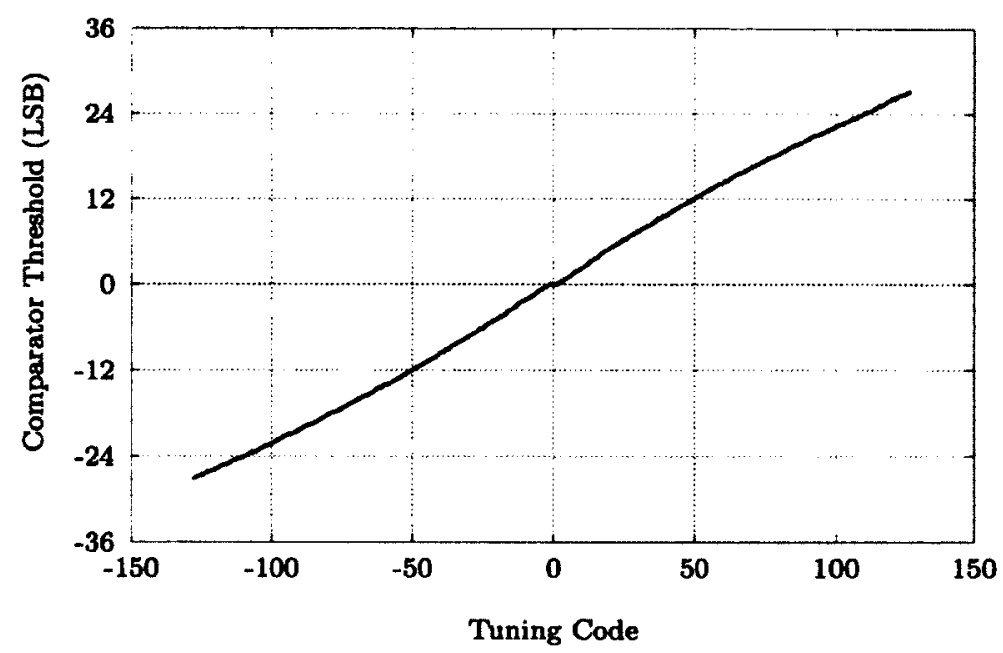

Figure 4.22: Threshold variation as a function of the coarse array tuning codes, positive codes enable array CRSB connected to the negative output node; $\mathrm{f}_{\mathrm{S}}=80 \mathrm{MHz}, \mathrm{V}_{\mathrm{IN}}=1 \mathrm{LSB}$

for any measured offset, typically corresponding to $200 \mu \mathrm{V}$ or $8.3 \%$ of an LSB. 


\subsubsection{Layout of SCMTAs}

The layout of the comparator is presented in Fig. 4.12. The annotated layout Fig. 4.12 differentiates between the fine tuning arrays and the coarse tuning arrays. The tuning lines $\mathrm{X}_{\mathrm{P}}, \mathrm{X}_{\mathrm{N}}$ run on the third metal layers closer to the power rails. The tuning lines responsible for the coarse tuning labeled $\mathrm{O}_{\mathrm{P}}, \mathrm{X}_{\mathrm{N}}$ run through the center of the cell. Ideally the tuning lines should be placed far from the power supply traces, preferably on a higher metallization layer. Since the tuning cell is connected through 22 lines to the shift register memory cell, metallization layers are exceedingly dense leaving little choice in the metallization option for the tuning lines which are connected to the comparator nodes.

Fortunately, during the typical operating scenario, the shift register is not operating during a comparison. As such there should be considerably less digital noise being coupled into the power supply rails. Great care was taken when routing the CLK signals with respect to this design consideration as is presented in Sec. 4.7. The SCMTA block occupies a total IC real estate area of $224.8 \mu \mathrm{m}^{2}$. 


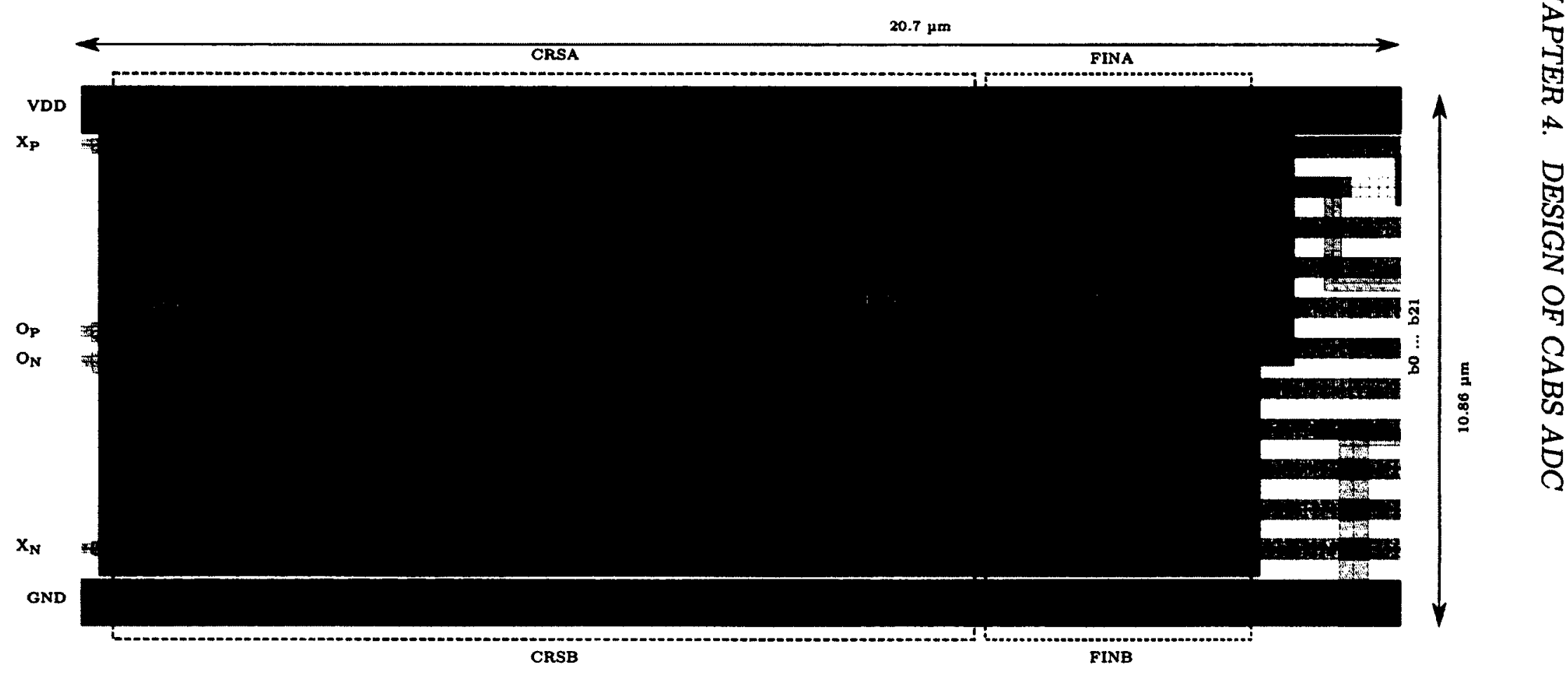

Figure 4.23: SCMTA Layout, CRSB refers to the coarse tuning array on the positive input side of the comparator connected to $\mathrm{O}_{\mathrm{P} S}$, FINB is the fine tuning array on the positive input side connected to the regeneration node $\mathrm{X}_{\mathrm{N}}$. 


\subsection{Shift Register Based Memory}

The SCMTA array used to establish the required thresholds in the last four stages of the ADC necessitates 22 parallel control signals for the coarse and the fine tuning arrays. To create these control signals, write-enable D flip-flops (WE-DFF) are connected sequentially into a shift register.

When the ADC is undergoing calibration, the comparator tuning code is propagated into the shift register one bit at a time. Assuming a low clocking frequency of $3 \mathrm{MHz}$ it takes $7.3 \mu \mathrm{s}$ to load the data into one 22 bit register or $1.76 \mathrm{~ms}$ for the entire 5280 bit shift register. Calibration and programming are performed off-line and do not interfere with the conversion process contributing little to the overall small dynamic power consumption of the ADC.

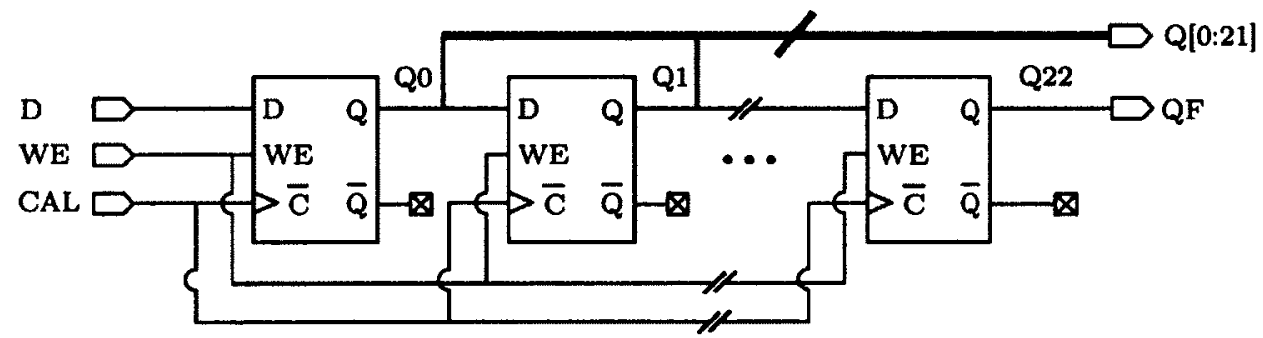

Figure 4.24: Shift register used for the control signal of the SCMTA tuning cell, each shift register is 22 bits long, "D", "CAL" and "WE" are the data, calibration clock, and the write enable signals, respectively, QF is the final output and feeds into the " $\mathrm{D}$ ", data of the following 22 bit register.

An illustration of the 22 bit shift register is presented in Fig. 4.24. The output "Q[0:21]" is bus connected to the four separate tuning arrays contained within the SCMTA cell. The design of the digital cells used for the shift register was kept hierarchical to prevent excessive re-design or the creation of banks of digital cells with varying drive strength capabilities. Hierarchical design lends itself to ease of reusability at the expense of increased real estate. The shift register is built out of 
write-enable rising edge D flip-flops. The flip flops themselves are built from d-latches which in turn are based on transmission gates. The design of the d-latch was adopted from [38]. For similar designs of digital cells along with an in depth discussion of the benefits of transmission gates in reducing the device count for digital cells, the reader is encouraged to examine $[39,40]$. 


\subsubsection{Shift Register Layout}

The shift register was broken down into standard cells with the same height as the comparator and bitline driver as well as the SCMTA arrays. Each row formed a register macro cell with two versions, a feed forward cell, propagating the code from left to right, and a reverse feed cell propagating the code from right to left. The data "snakes" around the layout area until reaching the final shift register cell in the $8^{\text {th }}$ stage.

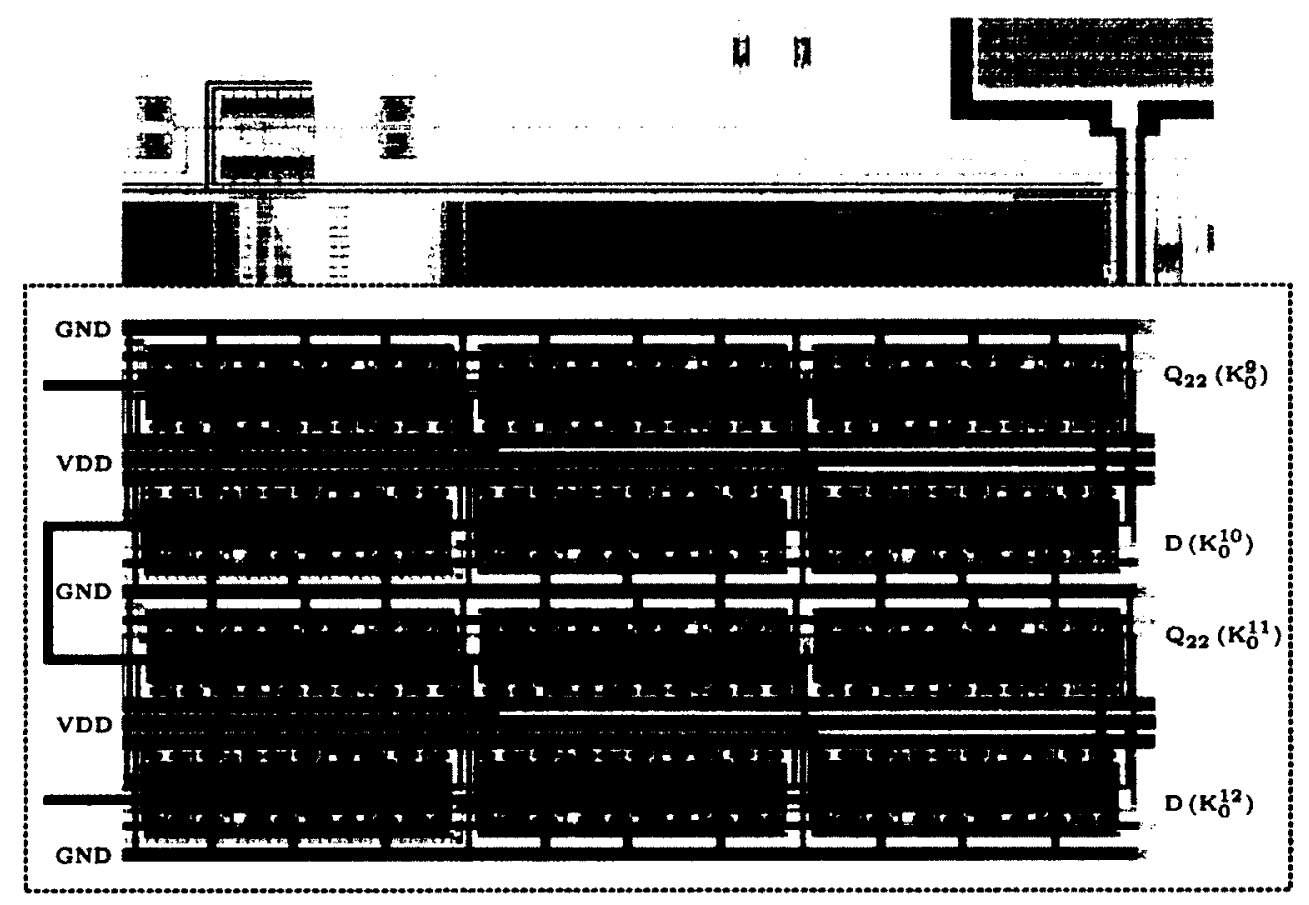

Figure 4.25: Layout of the shift register.

A close up of the shift register is presented in Fig. 4.28. The layout of the D flipflops was done in such a way as to allow for smooth abutting between any number of cells. The minimum standard cell height was determined by the shift register macro cell. The 22 bits require 22 nets to be brought to the SCMTA tuning block. These interconnects were routed on layer MQ and MG, corresponding to the fourth 
and fifth metallization layers. The minimum height was then determined by the minimum design rule spacing between the interconnects $0.40 \mu \mathrm{m}$ which is $8 \mu \mathrm{m}$ for 22 nets, as well as allowing $2 \mu \mathrm{m}$ for the VDD and GND power rails ${ }^{45}$. To reduce the overhead caused by power supply traces each macro cell shared its supply and ground rail with two adjacent macro cells (excluding the top and bottom macro cells).

The supply and ground rails were doubled, utilizing both M1 and M2 metal layers to reduce the effects of a voltage drop across the power rail of the macro cell when data is being loaded into the shift register. The maximum instantaneous current for a macro cell is $4.21 \mathrm{~mA}$. The voltage drop on the power supply from the first flip flop to the $22^{\text {nd }}$ flip flop is around $213 \mathrm{mV}^{46}$. The value is calculated based on the sheet resistance values in [35].

\footnotetext{
${ }^{45}$ Routing the control signal over or near the power supply lines was avoided in the interest of reducing the possibly of coupling from the power lines into the controls signals. Recall Sec. 4.3, any variation in the control signal changes the effective capacitance of the capacitive tuning device.

${ }^{46}$ Note that this value was based on simulations without extracted layout parasites and capacitances, as a consequence all the D-flip-flops are triggered instantly as data is fed through the register, over estimating the instantaneous maximum power draw.
} 


\subsection{Output Buffer Design}

The input/ouput buffers used in the ASIC are also used in the distributed clock tree presented in Sec. 4.6. The test structure used in Fig. 4.26 was used to remove the idealized driving power of the signal source. Capacitances are introduced between the input nodes of the buffers to emulate the capacitive loading of interconnect and via structures ${ }^{47}$. A loading capacitance of $150 \mathrm{fF}$ was used at the buffer input nodes. The full bondpad model with associated ESD protection was included as well as the inductance and capacitance contributed by the package. An inductor of $2 \mathrm{nH}$ was used for the load impedance along with a capacitance of $15 \mathrm{pF}$ for a rise time of approximately $1.3 \mathrm{~ns}$ or $3.2 \%$ of the sampling period.

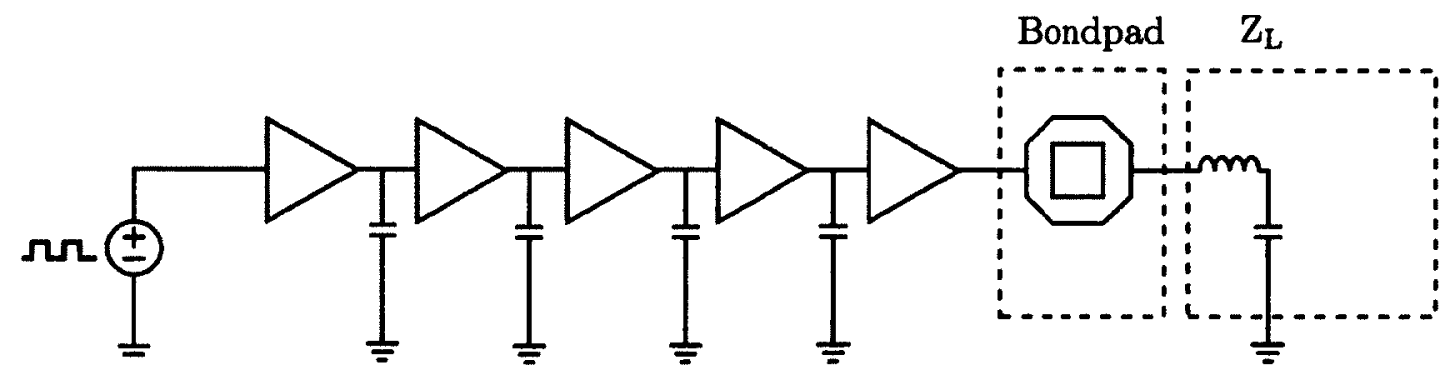

Figure 4.26: Test bench schematic for output buffer design.

The schematic of the output buffers is presented Fig. 4.27 along with the device sizes in $T b$. 4.4. The classic work presented in [41] for the design of tapered buffers points to a 2 stage tapered design for the input capacitance of the smallest inverter in the buffer chain with a capacitance of $37.5 \mathrm{fF}$.

\footnotetext{
${ }^{47}$ Resistance value of the lines were not critical in this application, a metal interconnect on E1 with $100 \mu \mathrm{m}$ and minimum width has a resistance of only $0.4 \mathrm{Ohms}$.
} 


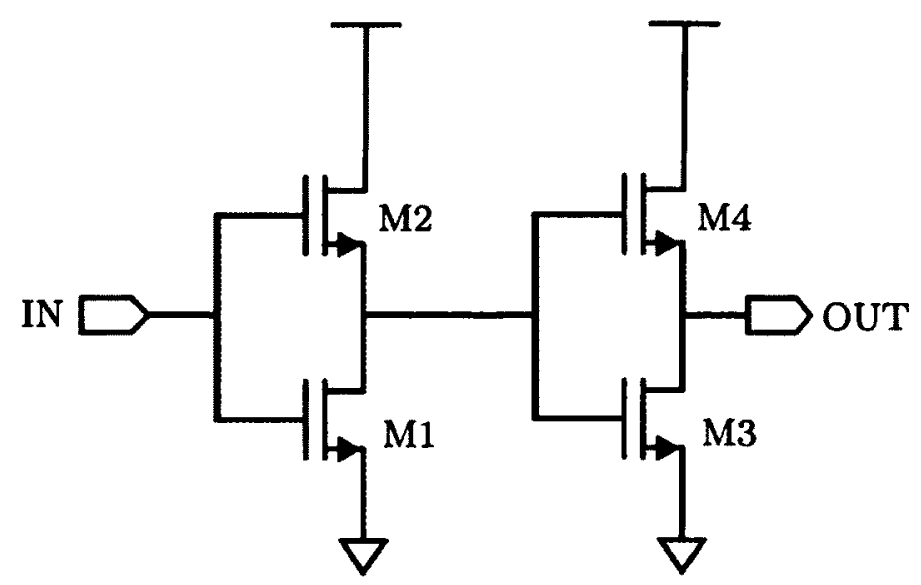

Figure 4.27: Schematic diagram of output buffers.

Table 4.4: Device sizes for the output buffer.

\begin{tabular}{cccc}
\hline Transistor & Width $\mu \mathrm{m}$ & Length $\mathrm{nm}$ & Fingers \\
\hline M1 & 3 & 120 & 4 \\
M2 & 12 & 120 & 4 \\
M3 & 16 & 120 & 16 \\
M4 & 48 & 120 & 20 \\
\hline
\end{tabular}

\subsubsection{Output Buffer Layout}

The layout of the output buffer is presented in Fig. 4.28, the input and output contacts are both on the $\mathrm{E} 1$ metallization layer. A tie down is connected to the input of the buffer to prevent antenna rule violations during processing. As well, a double guard ring has been included around the buffer to reduce large switching noise from being injected into the substrate. 


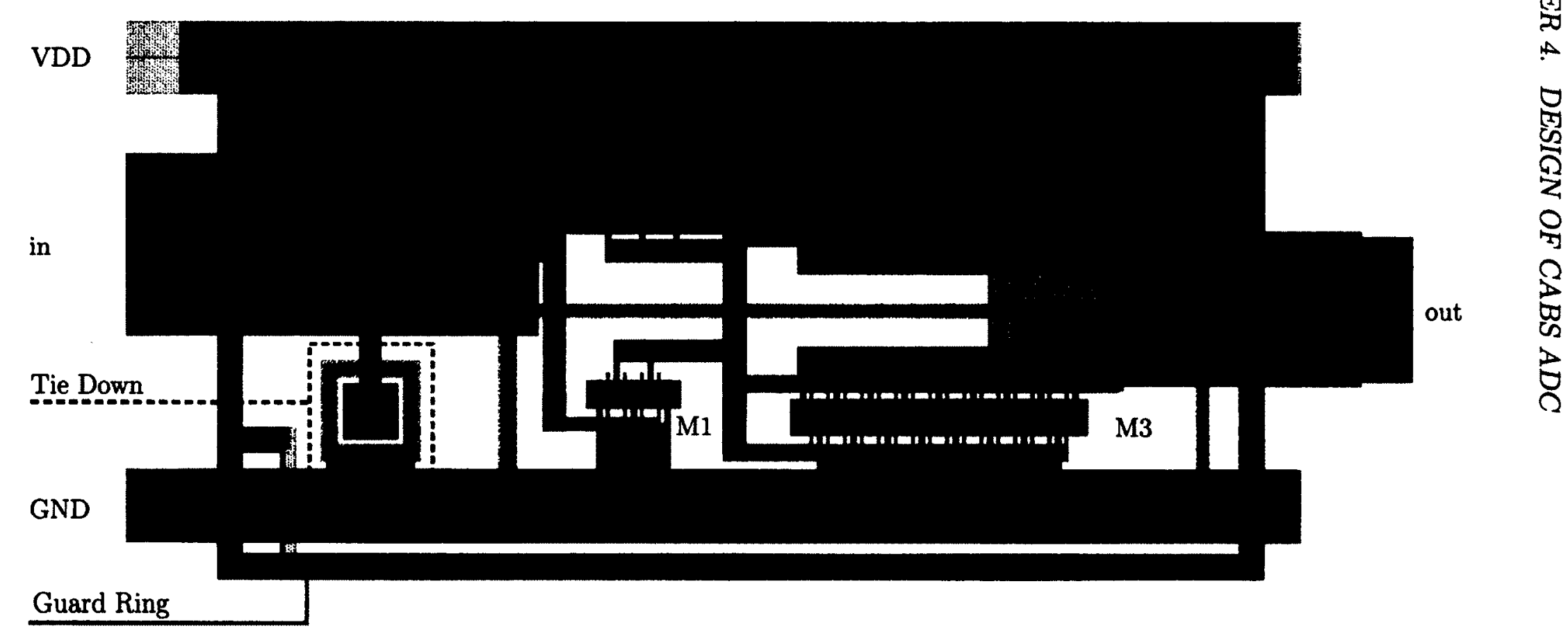

Figure 4.28: Layout of the output buffer, double guard ring (n-well) and (p-well), input/output contacts on the $5^{\text {th }}$ metallization layer, dual layer power supply rails. 


\subsection{Clock Tree}

The input capacitance for the calibration clock "CAL", "WE", and "D" input pins was measured for the WE-DFF and is presented in $\mathrm{Tb}$. 4.5. For a row macro cell containing 22 flip flops the worst case input capacitance for the CAL line is around $325 \mathrm{fF}$. In addition the trace contributes an extra $160 \mathrm{fF}$ for a total capacitance of $458 \mathrm{fF}$. As a rough approximation, operating without a clock tree, the input capacitance would be $109 \mathrm{pF}$ for the CLK input to the entire shift register. Such a large capacitance is impractical on IC, increasing the duration required for loading data into the shift register as well as requiring unrealistically large and power hungry buffers to obtain reasonable operating frequencies.

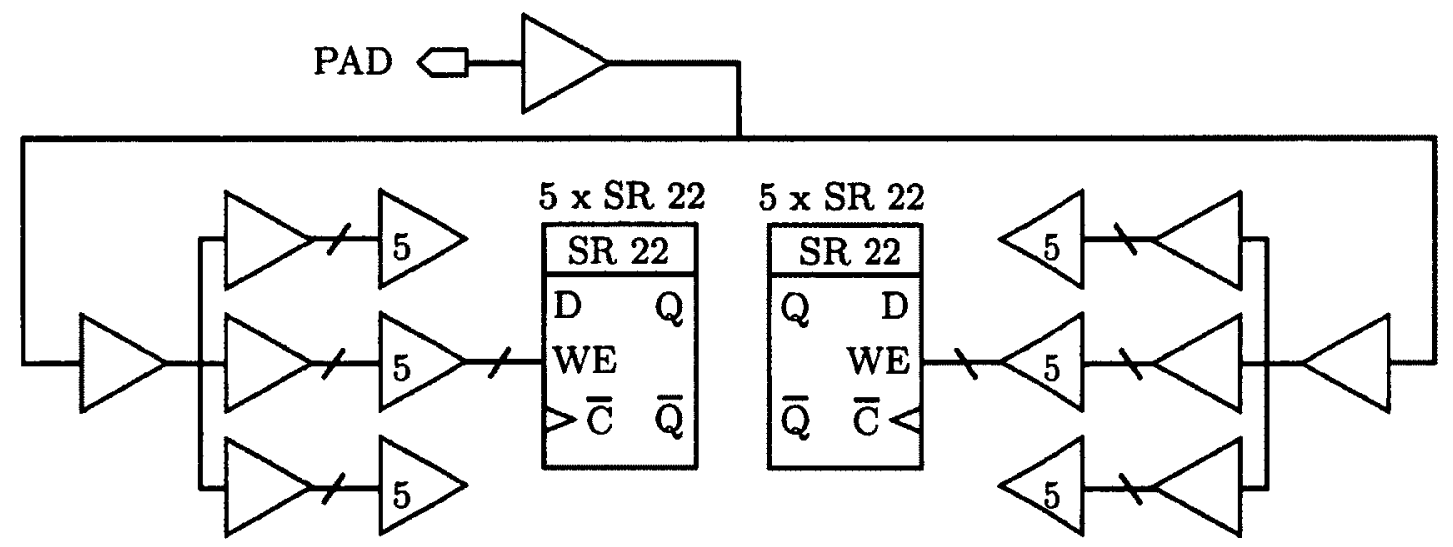

Stage 1 Stage 2 Stage 3

Left Side

Right Side

Figure 4.29: Clock tree with three levels of corresponding hierarchy.

Consequently, a clock tree was designed to distribute the "CLK" signal to the 240 shift register cells. A simple clock tree structure pictured in Fig. 4.29 is used for both the "WE" and "CAL" signals. Given the large metallization density of the shift 
register ${ }^{48}$ the root style structure was placed on the sides of the IC as discussed in Sec. 4.7. The data signal has only one buffer from the PAD to the input of the shift register as it is loaded only by the trace capacitance and the $1.8 \mathrm{fF}$ input of the first DFF.

Table 4.5: Input capacitance of WE-DFF.

\begin{tabular}{cc}
\hline Pin & Capacitance (fF) \\
\hline CLK & 14.5 \\
WE & 7.1 \\
D & 1.8 \\
\hline
\end{tabular}

Once the input capacitances were measured, a behavioral model was created. The behavioral model replaced trace impedances by their pi-model equivalents $[38,42]$ and the input impedance of the flip flops by ideal capacitances ${ }^{49}$. As shown in Fig. 4.29, a first stage buffer drives three second stage buffers. In turn each second stage buffer drives five individual buffers in the third stage. Finally, a buffer in the third stage is responsible for driving the signal into the eight rows of shift register macro cells. This structure is symmetrically repeated on both sides of the IC, the first stage buffers belonging to both clock trees (left and right side of the IC) are driven by a single buffer from the pad. All the buffers are the same size as Sec. 4.5.

\footnotetext{
${ }^{48}$ In a row macro cell, the entirety of the first five metal layers (M1, M2, M3, MQ, MG) are used to propagate the data or establish the tuning bits. As such it would be difficult to insert contacts to a higher metal layer such as $\mathrm{E} 1$ which requires a minimum $6 \mu \mathrm{m}$ by $3 \mu \mathrm{m}$ contact, there would have be a physical space between rows allowing for a contact.

${ }^{49}$ Note that there is a reasonable difference between lumped and distributed models. The rise time of "CLK" signal when driving an RLC load can be lower than a pure RC load. The reader is encouraged to examine [42] for an excellent presentation on interconnect design and simulation.
} 


\subsubsection{Clock Tree Layout}

A portion of the clock tree is shown in Fig. 4.30. The global signal arrives from a first stage buffer to the second stage buffer on metal layer "MA" the final metal layer in the process. The output of the second stage buffer is then connected to a line which feeds its five subsidiary children. In the "WE" and "CAL" lines are visible as they extend from the two separate clock trees to eight 22 bit registers. Notice, the clock trees themselves have their power lines abutted to save layout area. This is acceptable only because the "WE" signal changes only at the start and the end of a tuning code, the effect of coupling between the two lines is not significant.

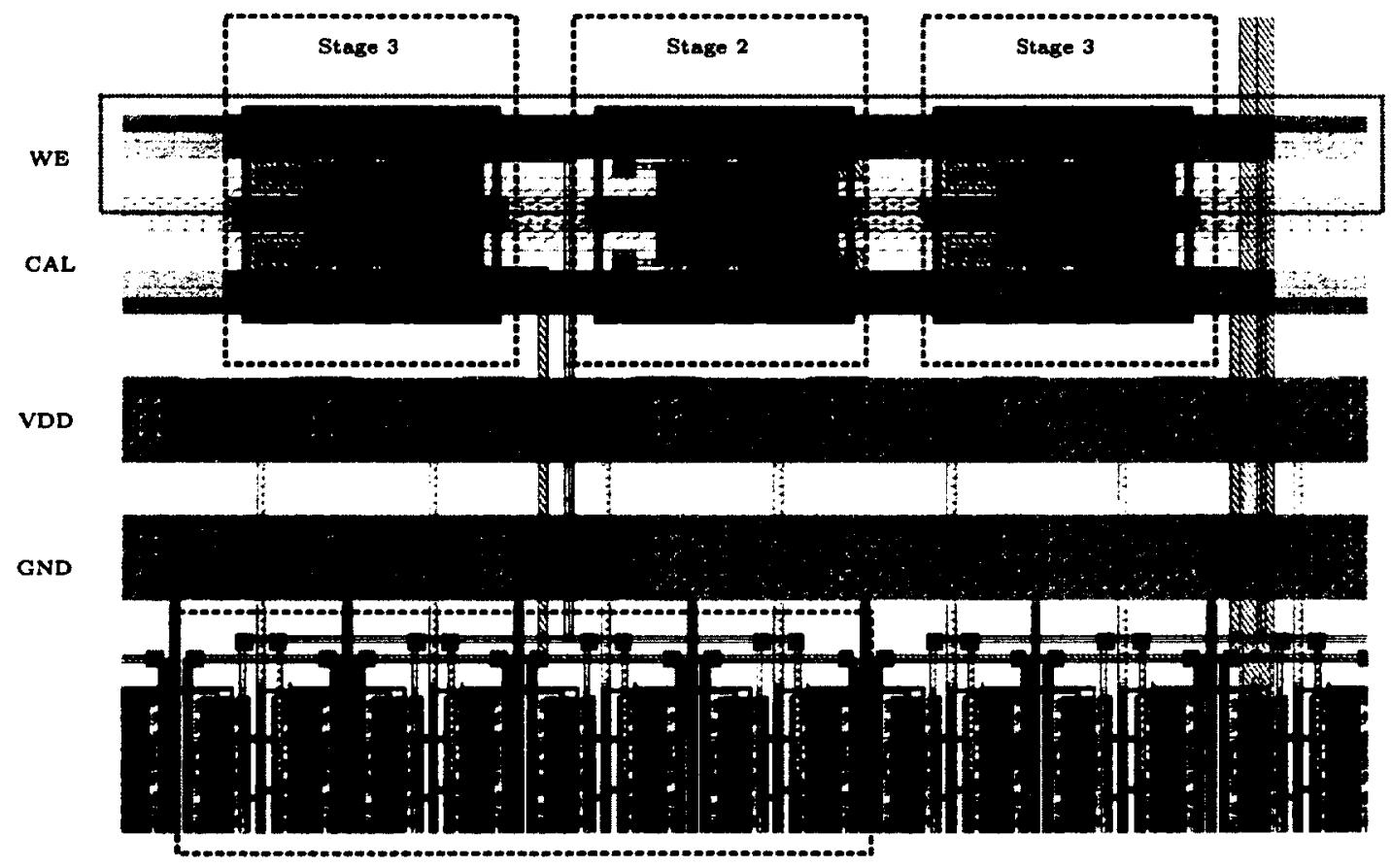

Figure 4.30: Closeup of a region containing the clock tree. 


\subsection{ASIC Layout Integration}

\subsubsection{Floor Planning}

In the design of this ASIC, the full library of analog and digital cells was designed from the ground up. As such when it came to the floor planning stage in the layout phase of the design, it was difficult to approximate dimensions of the various physical layout areas without creating an inial layout for the some of the standard cells. The floor plan which was followed throughout the design is presented in Fig. 4.31. The comparator and the WE-DFF were the first cells designed. Initially these cells took advantage of analog design techniques; guard rings and dummy cells in the case of the comparator 50. Based on a quick estimation including the size of the decoupling capacitors, the ESD devices, and the chip frame it became evident that the comparator and the DFF cell were too big $(14.41 \mu \mathrm{m})$ to accommodate 120 rows of macro cells on either size of the IC. The height of the standard cells were compressed further to $10.97 \mu \mathrm{m}$.

All the standard cells designed had a fixed height for the power lines, allowing for direct abutting of power lines. The DFF was designed to have all signals abut, that is to say the output of previous flip-flop "Q" is directly connected to the input "D" of a proceeding flip flop when the cells are abutted. All the cells maintain a standard left-to-right signal flow.

The floor plan was based on a signal-driven planning methodology [43]. The motivation behind using such an approach with a low frequency IC, $25 \mathrm{MHz}$ sample rate, lies with the large amount of asynchronous clock signals used to trigger the comparators in the 8 stages of the ADC. Over 254 interconnects are used as separate CLK nets for the eight stages of the ADC. The majority of those, 224 nets are present

\footnotetext{
${ }^{50}$ Common centroid technique was not used for the input differential pair since the devices are intentionally mismatched.
} 
in the tuned core.

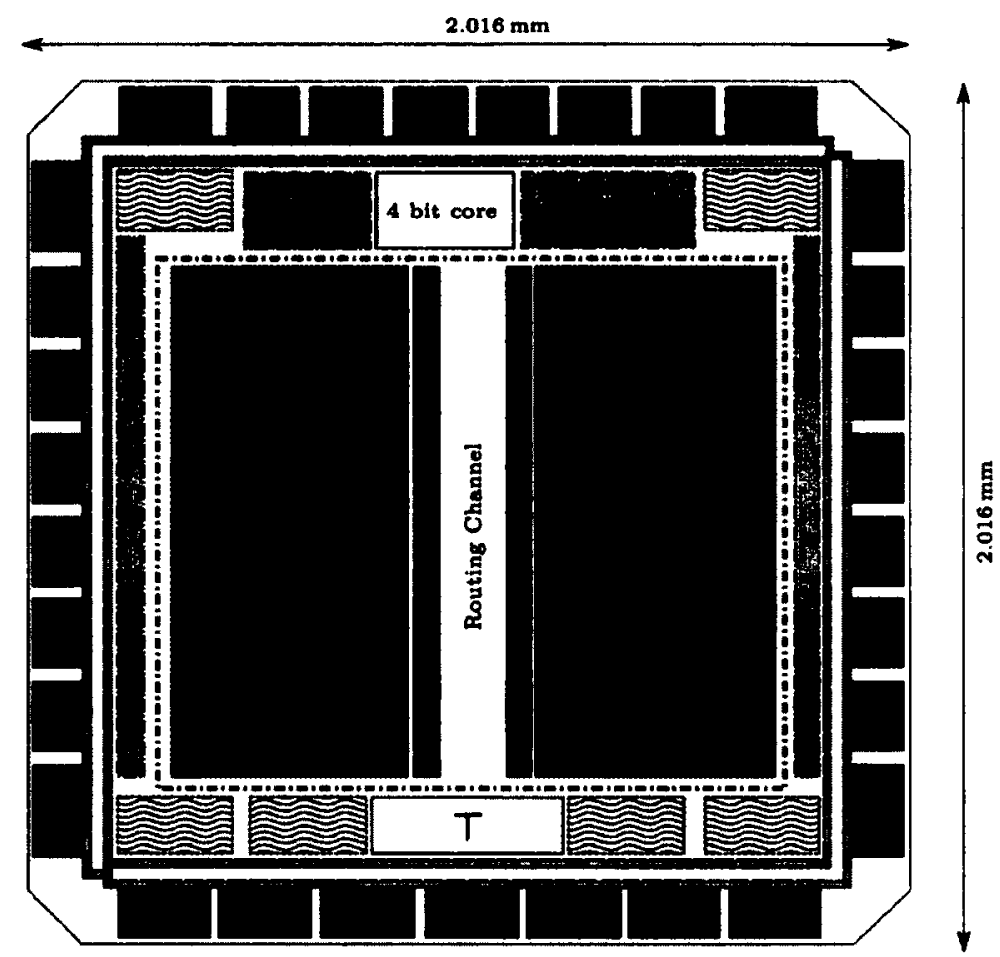

Analog Circuitry Shift Register Decoupling Capacitors $\square$ Power Ring 1 i. Power Ring 2 Test Structure

Figure 4.31: Layout floor plan for the ADC.

The most sensitive analog circuitry is located at the center of the IC with 120 row macro cells symmetrically centered around the routing channel. The routing channel carries all the asynchronous clock signals along with the differential input signals. The routing methodology is described with more depth in Sec. 4.7.3. The decoupling capacitors were placed as close as possible to the power supply pins. The core of the ADC (shift register, comparators, SCMTA, bitline drivers) is surrounded by a secondary power ring which is connected to separate supply pins. This was done to avoid coupling of digital noise through the power supply lines, originating from the input/output buffers. To avoid running the clock signal through the sensitive routing channel or the high density shift register, it was determined the best placement of the 
clock tree lies between the first and second power rings. Output buffers with their corresponding falling edge DFFs used to sample the bitlines are placed in the same areas as the clock tree, as is indicated in Fig. 4.31.

\subsubsection{4-Bit Hard Coded Core}

To increase the frequency of operation the first four most significant bits [b0..b3] are determined by a "hard coded core" (HCC). The HCC is in its own right a four bit CABS ADC. The layout of the HCC is presented in Fig. 4.32. It uses the same routing strategy as final tuned stages of the ADC. The output of the HCC is a 16 bit bus carrying the asynchronous clock signals which trigger the CLK inputs of the comparators in stage 5 ("b3"). The HCC occupies a total area of $10,803 \mu^{2}$ or $0.265 \%$ of the total area.

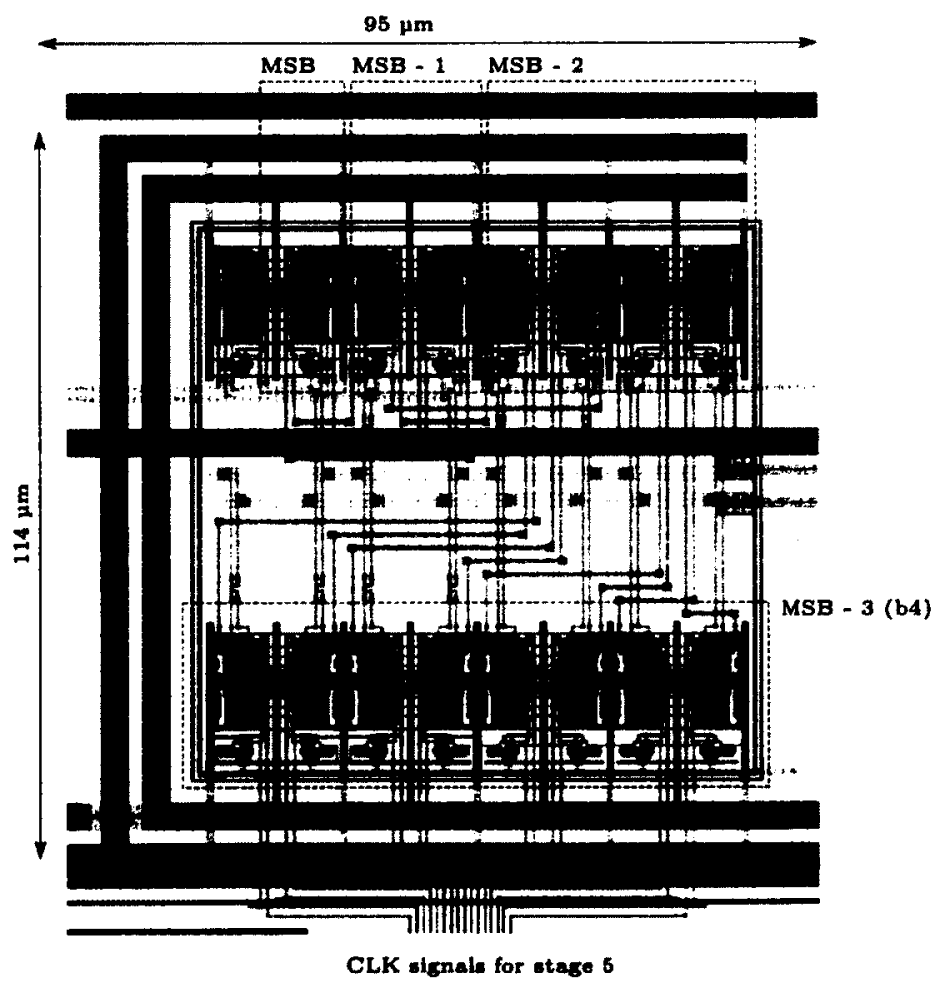

Figure 4.32: Layout of the 4 bit HCC. 


\subsubsection{Routing Methodology}

In Fig. 4.33 a close up of the routing channel reveals the high density of asynchronous clock lines. Metal layers MQ and MG were used to propagate the signals top-down through the channel. It is tempting to suggest using more metal layers to increase the distance between neighboring nets. The low metallization layers (M1, M2) have high sheet resistance and capacitance for the large routing channel distance of $1.2 \mathrm{~mm}$. Metal layer M3 is used to connect the CLK outputs/input of comparators in a stage to the routing channel. Metal layers above MG, specifically E1 and MA are too large to accommodate the number of nets required ${ }^{51}$.

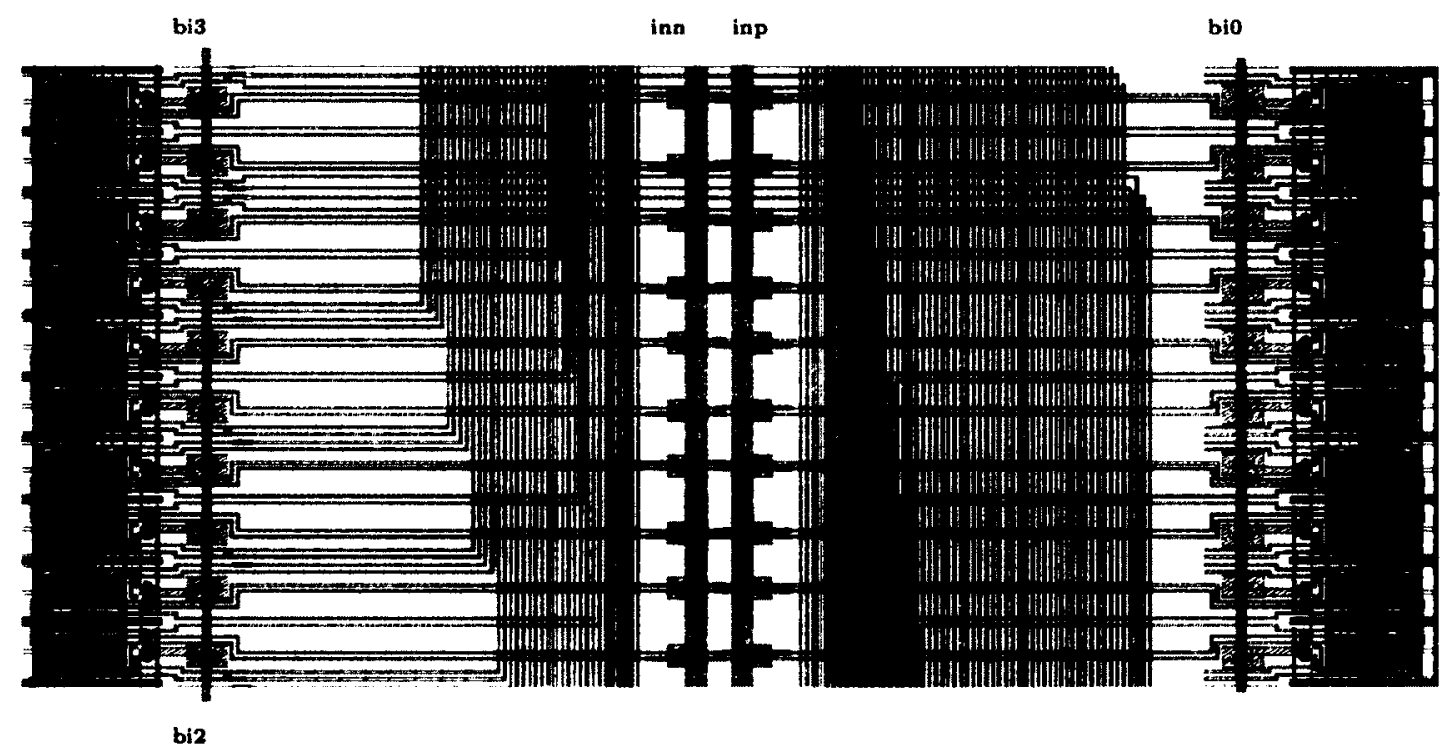

Figure 4.33: Closeup of the routing channel layout.

The differential input signal lines, "inp" and "inn" are routed on E1. A distance of $8 \mu \mathrm{m}$ is given between the differential nets and the nearest asynchronous comparator clock lines to reduce the effects of parasitic coupling. The differential lines themselves

\footnotetext{
${ }^{51}$ The aluminum "LY" metal layer was not used to route interconnect due to its high resistivity of $0.153 \Omega / \mu \mathrm{m}$ as opposed to $0.004 \Omega / \mu \mathrm{m}$ for $\mathrm{E} 1$.
} 
are spaced $3.7 \mu \mathrm{m}$ apart. Lastly the bitlines which hold the bit decisions upon sampling are connected close to the bitline drivers with contact arrays to reduce resistive loading. No global signals of any kind are routed on top of the routing channel.

\subsubsection{Power Supply Lines and Decoupling Capacitors}

As suggested in [44], power supply nets were one of the first considerations during the floor planning stage. The first power ring is used for the ESD devices, the clock tree and the input and output buffers. Row macro cells use a separate power ring. The individual cells have power supply lines on the first two metal layers. The width of the power supply lines is $1 \mu \mathrm{m}$ for both levels. Contact arrays were placed between the layers to reduce resistive coupling. Each row macro cell shares both the power supply and the ground rail with its neighbors above and below. An example of this is presented in Fig. 4.34. Notice, the power supply root (Power Ring 2) in the left side of figure is on three metal layers (M1,M2,E1) and feeding half of the ADC core.

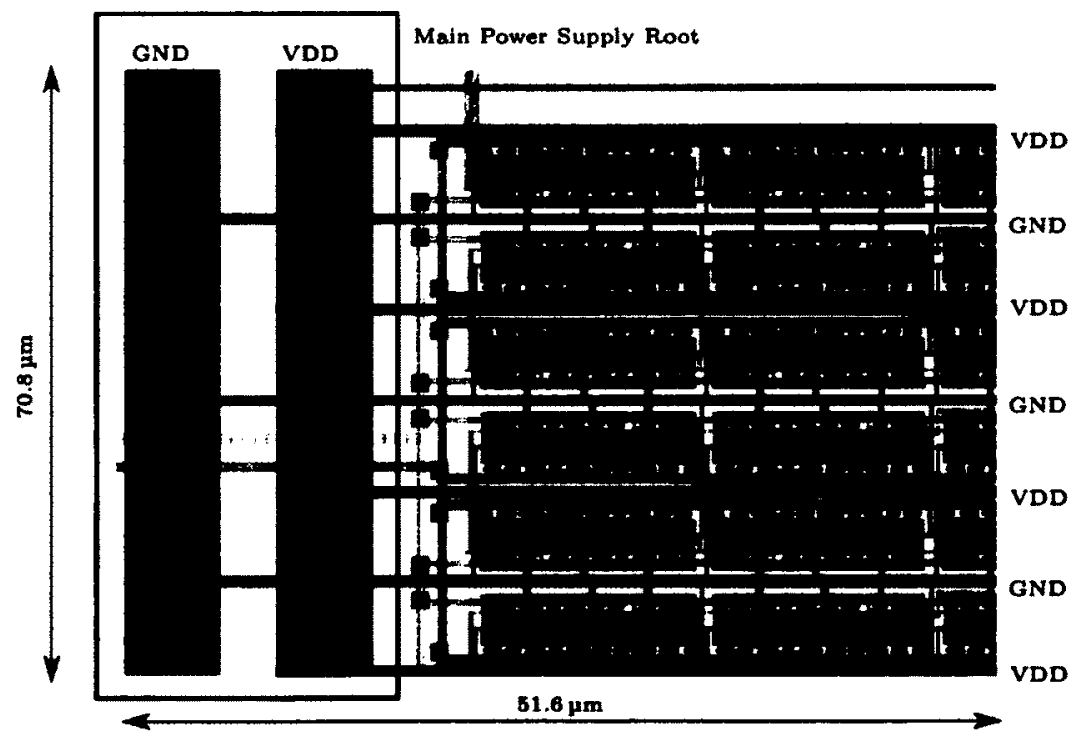

Figure 4.34: Layout of power supply lines feeding row macro cells. The power supply roots for "VDD" and "GND" are in the left side. Feeders extend to the row macro cells on the right. 
To ensure no cells were being current starved during the loading of the tuning code, the current handling capabilities of the different power supply lines were computed based on the equations in [35] and are presented in $\mathrm{Tb}$. 4.6. The equations in [35] are tedious and are based on empirical measurements and fitting analysis. Only the equations used to calculate the RMS and the DC current handling capabilities of M1 are presented in this section. When calculating the maximum current tolerance the corrected line width must be used to correct for process variations based on the width of the trace. For a trace on the first metallization layer M1 this corresponds to

$$
W_{c}=1.4+0.7 \cdot\left(W_{d}-1.4\right)
$$

for a metal trace wider than $1.8 \mu \mathrm{m}$ and less than $50 \mu \mathrm{m}$ wide. From this the maximum RMS current can be calculated with

$$
I_{r m s}=7.52(W-0.06) \sqrt{\left(1.19+\frac{3.53}{W-0.06}\right)} m A
$$

where the width " $\mathrm{W}$ " is in $\mu \mathrm{m}$ and can be either the corrected line width or the drawn line width. The DC current for M1 is given by

$$
I_{D C}=2.8(W-0.06) m A \text {. }
$$

Table 4.6: Maximum current tolerance for power supply nets.

\begin{tabular}{cccc}
\hline Power Net & Metals & $\mathrm{I}_{\max , \mathrm{rms}}$ & $\mathrm{I}_{\max , \mathrm{DC}}$ \\
\hline Row Macro Cell & $\mathrm{M} 1, \mathrm{M} 2$ & $30 \mathrm{~mA}$ & $5.5 \mathrm{~mA}$ \\
Power Ring 1 (chipframe) & $\mathrm{M} 1, \mathrm{M} 3$ & $78 \mathrm{~mA}$ & $31 \mathrm{~mA}$ \\
Power Ring 2 (core) & $\mathrm{M} 1, \mathrm{M} 2, \mathrm{E} 1$ & $300 \mathrm{~mA}$ & $217 \mathrm{~mA}$ \\
\hline
\end{tabular}


Decoupling capacitors are connected between the power rails to stabilize power supply voltages and reduce the amount of high frequency noise entering the IC from the external power supply. The decoupling capacitors used have a maximum capacitance of $200 \mathrm{pF}$ near DC. The type of decoupling capacitor is "ncap" which is an n-well based capacitor offering the most capacitance per area in this process. 


\subsubsection{Layout Approach Summary}

The full layout for the ADC is shown in Fig. 4.35. The metal fill is not shown to improve the clarity of the layout. Eight pads were used for the supply and ground signals for the second power ring, labeled "VDD" and "GND". The first power ring has a total of four pads for power and ground, labeled "VDDA" and "GNDA".

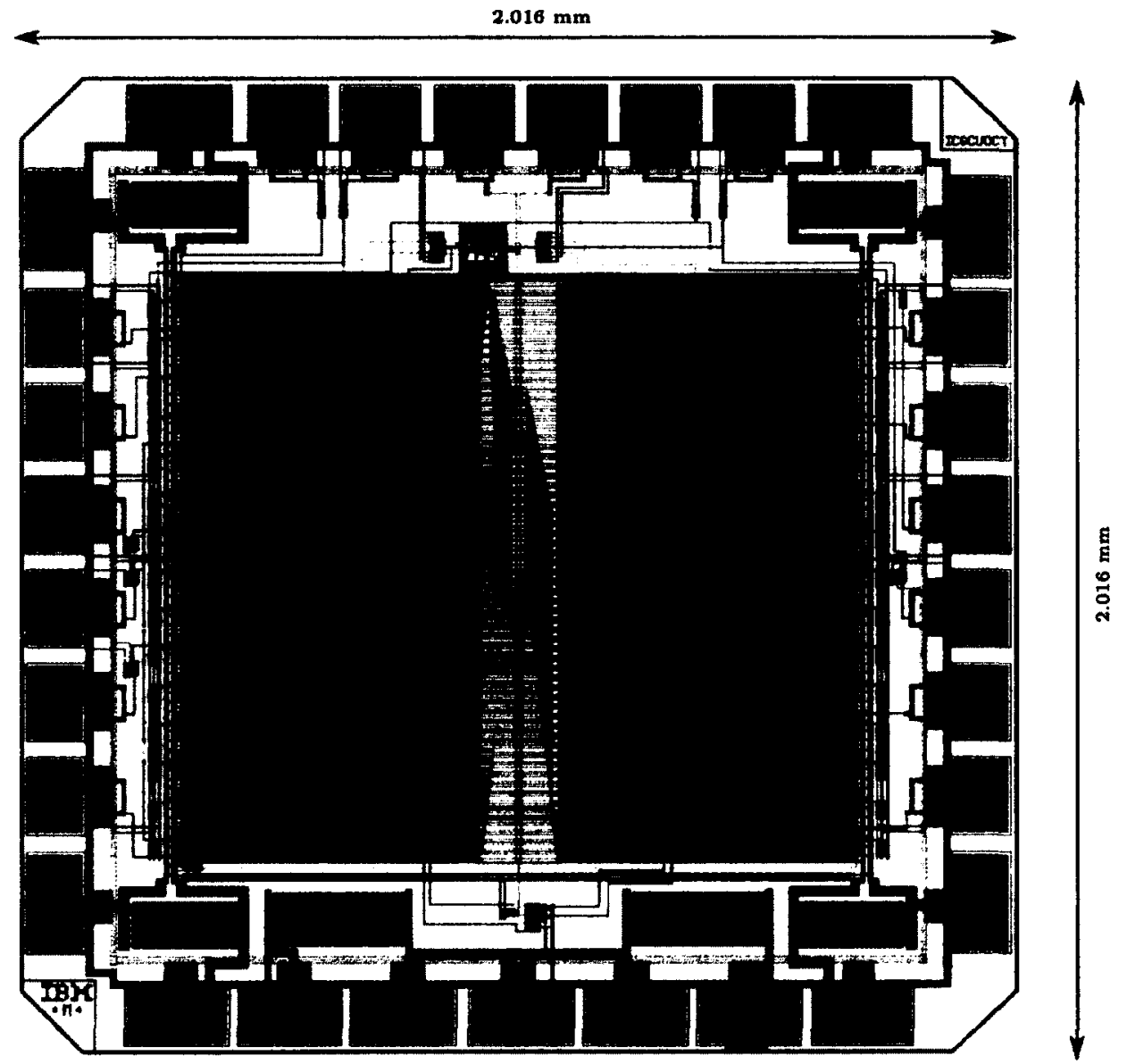

Figure 4.35: ICGCUOCT, CABS based ADC full IC layout without metal fill for clarity

The input pins "D", "CAL", and "WE" represent the data, calibration clock, and write-enable signals for the shift register. The global CLK signal is "CLK". The output bits are listed as [b7..b0]. A test structure was included which is a nominal 
comparator with the positive and negative output, "outp" and "outn". The bitline driver for the test device is "Bi". The signal "QF" reprents the final bit from the shift register and is used for verifying the tuning code integrity while the calibration code is loaded into the ADC. Only one PAD labeled "N/U" is left unused. 


\subsection{ADC Design Summary}

The design approach taken to construct an 8-bit exclusively CABS based ADC has been presented in Chap. 4 . The transient behavior of the comparator was analyzed to find two transient regions of operation ${ }^{52}$ which determine the total comparison time of the ADC. The comparison time ${ }^{53}$ then determines the upper limit on the frequency of operation since any comparison must be finished within $T_{\mathrm{g}} / 2$ before the bitlines are sampled on the falling edge of CLK signal. The bitline drivers were examined as well as their driving capability and their effects on the maximum frequency of operation.

The effect of PVT variation on the comparators was investigated; it was determined that a tuning method capable of handling $5 \sigma_{\mathrm{os}}$ of variation for the comparator offset must be used as a design criterion to guarantee the overall performance of the $\mathrm{ADC}^{54}$. The analysis of device mistmatch was then reversed, using device mismatch intentionally to shift the trip point of the comparator, shifting the threshold.

To reduce the ammount of custom designed cells and simplify the tuning algorithm a set of 12 IDWI devices was created. A method of tuning the threshold of the comparator around any established threshold was then discussed. The approach used pFET based MOSCAP capacitors operating deep in the accumulation region, connected to the output and regeneration nodes of the comparator. The programmable devices are then asymmetrically used to change the capacitance on the nodes shifting the threshold of the comparators. The SCMTA method was to establish threshold around a IDWI comparator cell as well as correct for PVT fluctuation in the offset value.

As well as the design of circuit elements, the layout and the approach taken to

\footnotetext{
${ }^{52}$ The set-up phase (Phase A) and the regeneration phase (Phase B).

${ }^{53}$ Here we consider the comparison time as delay from the $50 \%$ to $50 \%$ delay from the CLK input to the latched output bit going high for a positive bit transition.

${ }^{54}$ That is to say, guarantee near INL and DNL specifications.
} 
generate a systematic ASIC, standard cell library was presented, connecting circuit level decision with global layout constraints. An overview of the global ADC design choices as they relate to the discussion presented in this chapter and ADC performance metrics discussed in the following chapters is given in Sec. 5.4. 


\section{Chapter 5}

\section{ADC Metrics and Simulation}

\subsection{Static Linearity Analysis}

\subsubsection{Integral Nonlinearity (INL)}

The integral nonlinearity (INL), is defined as the deviation of a measured or simulated transfer characteristic of an ADC from an ideal linear fit ignoring quantization, gain, and offset errors. It is a measure of the "straightness" of the transfer function [1]. Although other techniques exist for the measurement of the INL, such as the histogram method [1] the "slow ramp" method was used measure the transfer characteristic of the $\mathrm{ADC}^{55}$.

Two slow ramps of $30 \mu$ s were used for a clock frequency of $30 \mathrm{MHz}$. The differential ramps were swept from $750 \mathrm{mV}$ and $450 \mathrm{mV}$ on the negative and positive input node to $450 \mathrm{mV}$ to $750 \mathrm{mV}$ on the negative and positive node covering the full $600 \mathrm{mV}$ differential input range. Each code is then sampled approximately 7 times for the ramp input frequency of $1.66 \mathrm{kHz}$. The sampled output bits of the ADC are presented in Fig. 5.1.

\footnotetext{
${ }^{55}$ The histogram method does not guarantee the monotonicity of the ADC.
} 


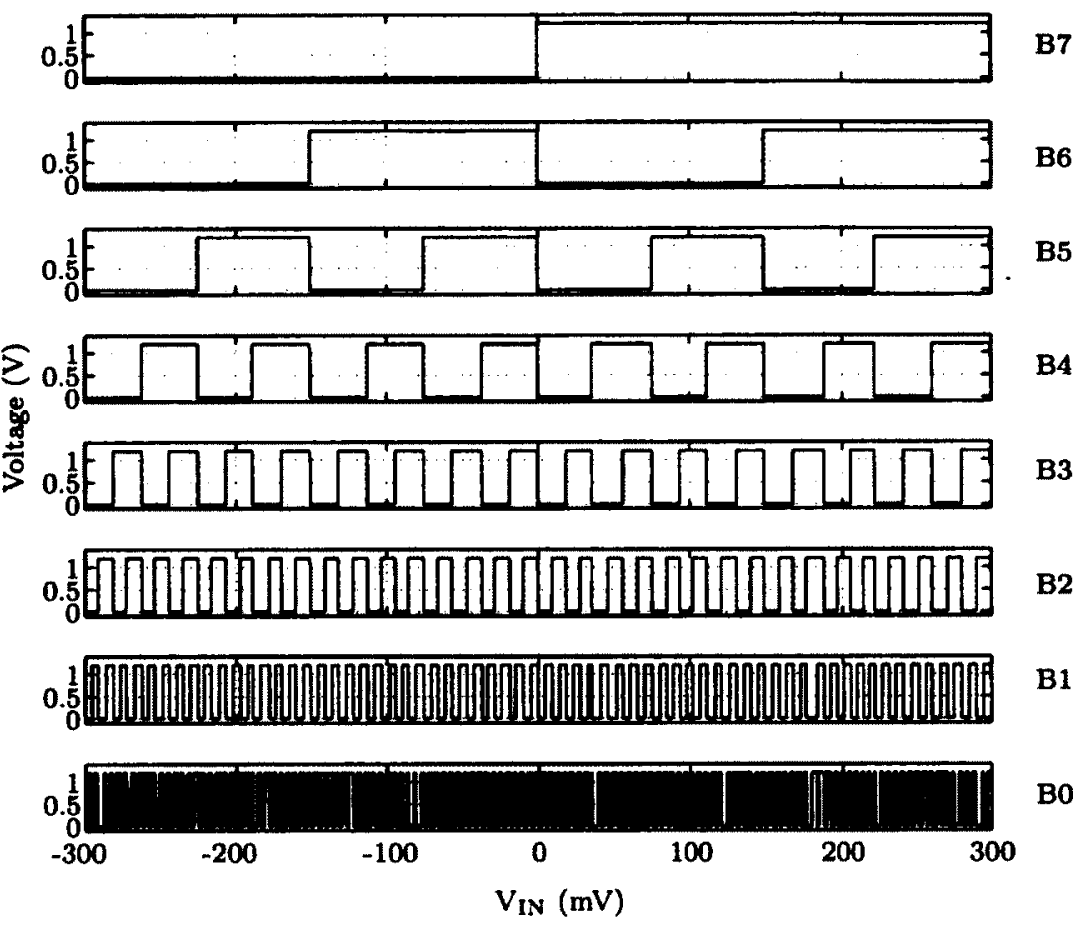

Figure 5.1: Sampled output bits, $\mathrm{f}_{\mathrm{S}}=30 \mathrm{MHz}, 30 \mu$ s differential ramp, $\mathrm{T}=90^{\circ} \mathrm{C}$.

Although the full transfer curve of the ADC is presented in Fig. 5.3 a portion of the curve is presented in Fig. 5.2 to underscore the methods used to find the INL and the DNL for the ADC. To obtain the straight line characteristic curve of the ADC the code transition points were used instead of code centers for measuring both the INL and DNL. Code centers are in the center of the "code width" as shown in Fig. 5.2. Another advantage of using the code transitions is that the DNL for a specific code is the difference between adjacent code transitions ${ }^{56}$.

Using code transitions paints a more accurate description of the INL and DNL of the ADC [1] avoiding unrealistic INL results with alternating high or wide codes. The transfer function of the ADC is obtained by passing the output of the ADC presented in Fig. 5.1 through an ideal DAC implemented in software.

\footnotetext{
${ }^{56}$ Adjacent code transitions are the transitions of the measured/simulated transfer function and the ideal transfer function transitions.
} 


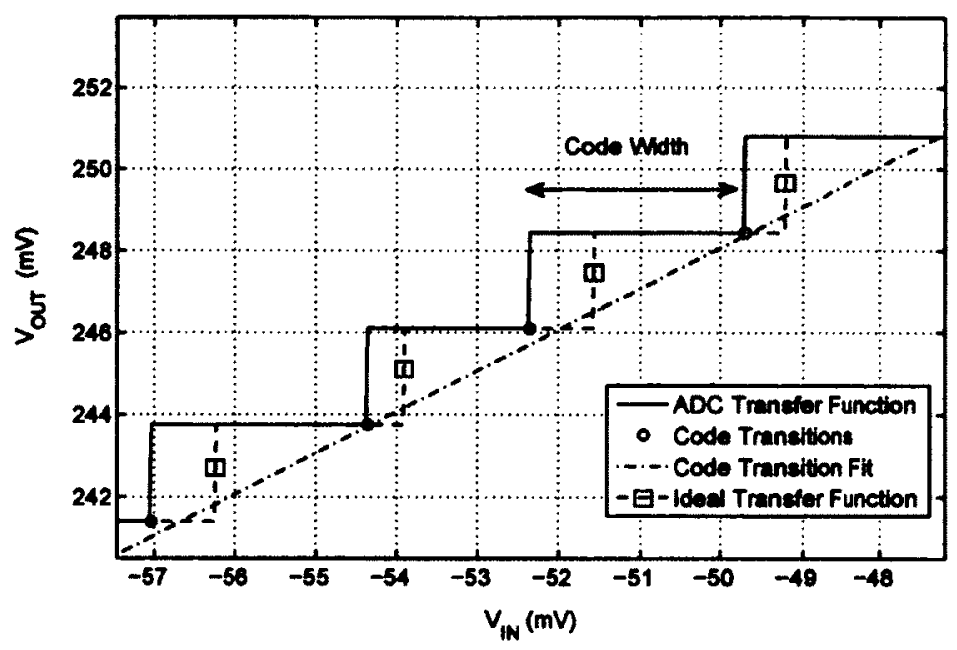

Figure 5.2: Magnification of the $A D C$ transfer function, code transitions and associated fit, ideal transfer function curve; $\mathrm{f}_{\mathrm{S}}=30 \mathrm{MHz}, 30 \mu$ s differential ramp, $\mathrm{T}$ $=90^{\circ} \mathrm{C}$.

The full transfer curve is presented in Fig. 5.3 for the sake of completeness. The input has been left in terms of the differential input.

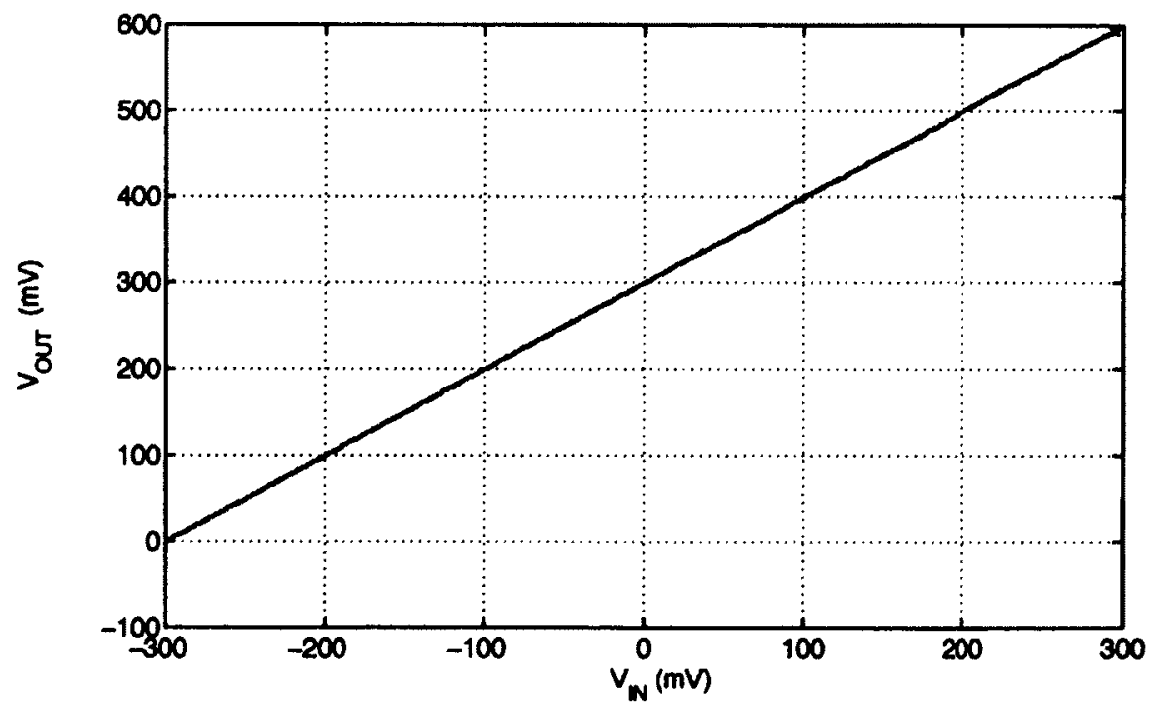

Figure 5.3: Full transfer function of the ADC with an overlay of the transition code best fit line; $\mathrm{f}_{\mathrm{S}}=30 \mathrm{MHz}, 30 \mu$ s differential ramp.

The INL is obtained by taking the difference between the transfer function of the 
ADC and the code transition fit line, presented in Fig. 5.4. The INL is -0.483 LSB for the simulation parameters discussed above.

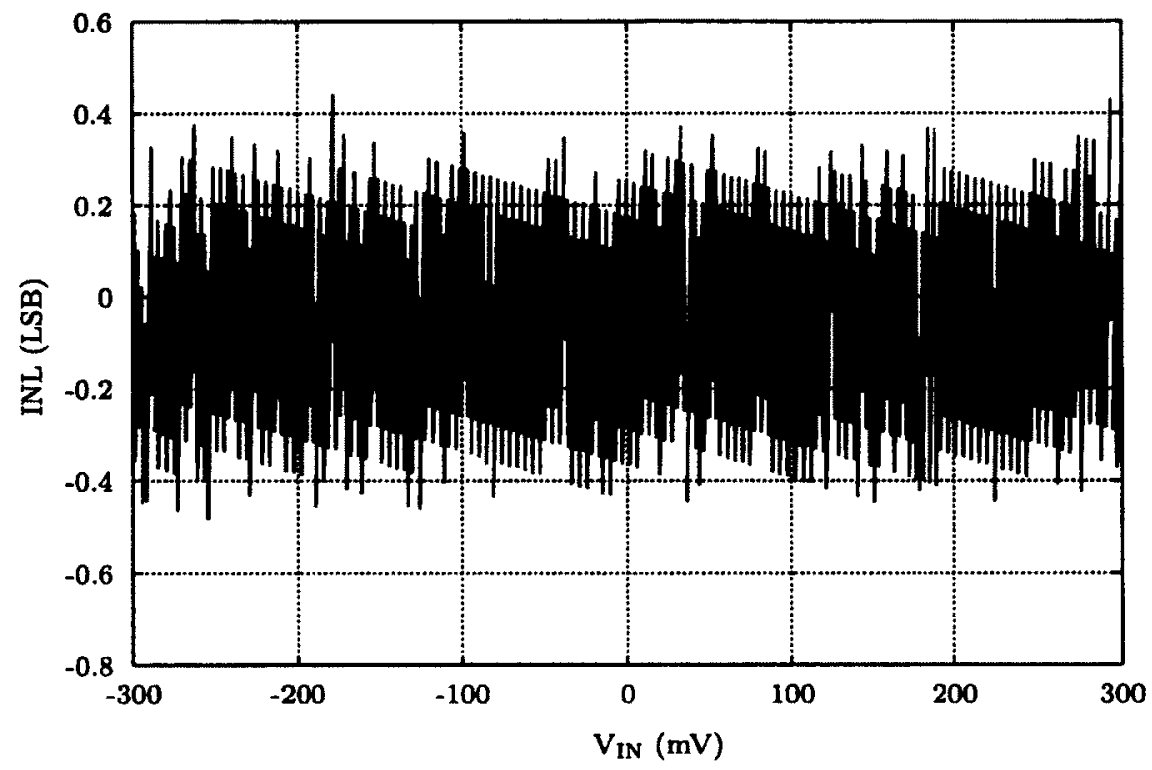

Figure 5.4: Integral nonlinearity (INL) as a function of input voltage $V_{I N}$, worst case INL is $-0.483 \mathrm{LSB} ; \mathrm{f}_{\mathrm{S}}=30 \mathrm{MHz}, 30 \mu$ s differential ramp.

At these operating conditions the ADC is not experiencing missing codes, the INL can be further improved by tuning the adjustable thresholds of the comparators.

\subsubsection{Differential Nonlinearity (DNL)}

The maximum acceptable DNL (excluding specialized applications or extreme regions of operation) allowed for an ADC is 1 LSB for an ADC without an intentional offset of $\mathrm{LSB} / 2$. A DNL larger than LSB/2 implies that there are input voltages for which the ADC will produce the same output resulting in a missing code. The DNL is presented in Fig. 5.5. The worst case DNL is $-0.4302 \mathrm{LSB}$ well bellow the requirement of LSB/2 for nominal simulation parameters and an intentionally offset INL/DNL measurement by LSB/2. 


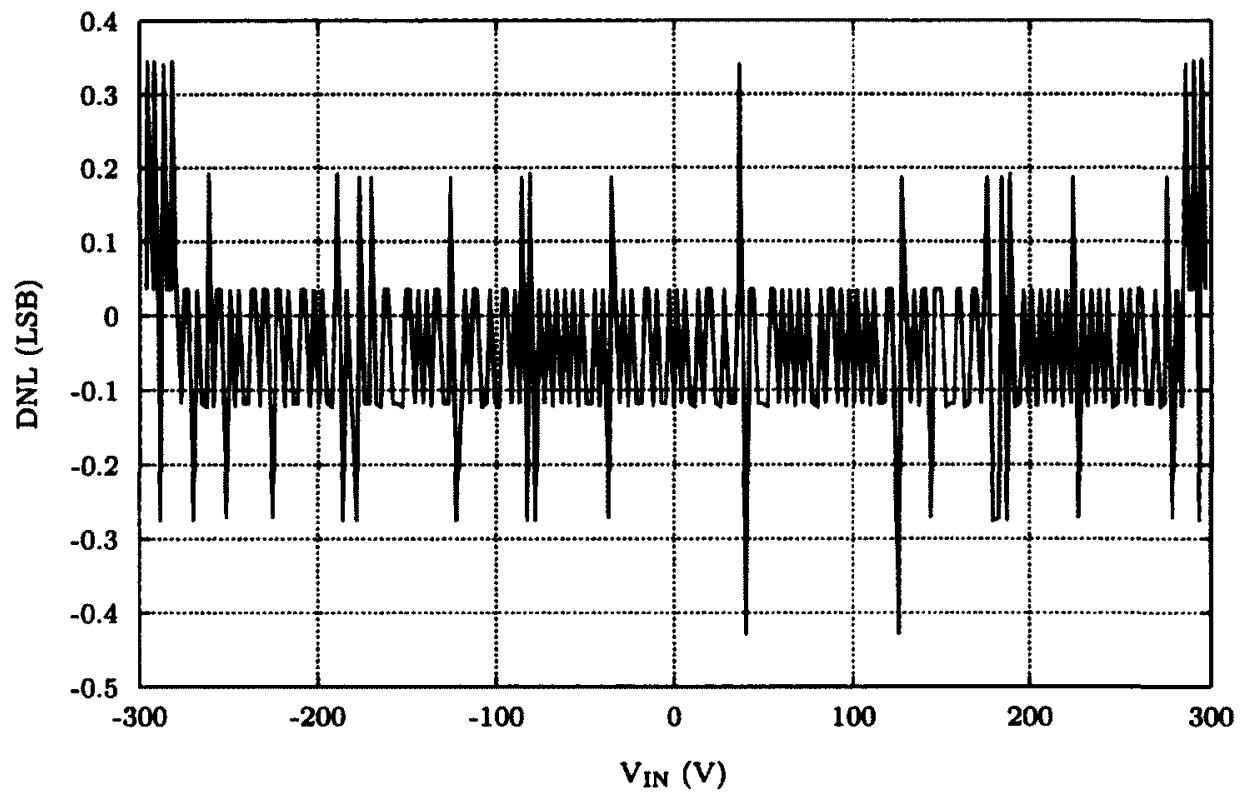

Figure 5.5: Differential nonlinearity (DNL) obtained using the difference between code transition; $\mathrm{f}_{\mathrm{S}}=30 \mathrm{MHz}, 30 \mu$ s differential ramp.

\subsubsection{Effects of Netlist Complexity on Simulation Time}

With 240 adjustable tunable comparators (including the shift register necessary for simulation) a $30 \mathrm{~ns}$ simulation required for the verification of one tuning threshold requires approximately 20 minutes, including netlisting and other aspects of simulation start up time. Naturally this analysis is performed only for positive threshold comparators, negative threshold comparators have symmetric tuning codes. A matlab script was written to generate OCEAN scripts ${ }^{57}$, simulate the offset and adjust the threshold to the desired level. In order to speed up simulation time the loading conditions on one comparator were mimicked with only 4 comparators in a simulation environment instead of using the full ADC. Through this process tuning a comparator threshold takes about 15 minutes. Given the large number of comparators it takes about 30 hours to tune all threshold to the desired levels (through simulation). The INL/DNL static analysis, lasts a duration of 50 hours for a total of 80 hours for

\footnotetext{
${ }^{57}$ OCEAN script is the scripting language used in Cadence Tools.
} 
threshold adjust and static analysis simulation. As such it is impractical to perform Monte Carlo analysis on the INL and DNL and instead is it is necessary to rely on the component level based Monte Carlo ${ }^{58}$ analysis for an estimation of the performance of the ADC under process variation instead of global ADC monte carlo simulation.

${ }^{58}$ Such as the Monte Carlo analysis for a tuned row macro cell. 


\subsection{Dynamic Analysis}

Through its conversion, the ADC assigns a range of voltage levels to a particular quantized level. Spectrally, this uncertainty appears as a random noise [45]. Assuming a uniform distribution of $\operatorname{codes}^{59}$ the noise contributed to the analog signal by the quantization noise is given by:

$$
V_{n}=\frac{L S B}{\sqrt{12}}=0.664 \mathrm{mV}
$$

To obtain the ADC's dynamic performance metrics, a single sinusoidal tone $\mathrm{e}^{60}$ was used for the input to the ADC. The output of the ADC was then passed through an ideal DAC and converted back to an analog signal. Using coherent sampling an FFT was carried out on the reconstructed analog signal to obtain the frequency spectrum. With the spectral information it was then possible to find the dynamic metrics such as SINAD, THD, SNR, SFDR and ENOB. The FFT itself has a theoretical noise floor given by

$$
N F F T_{d B}=10 \cdot \log _{10}(M / 2)
$$

where " $M$ " is the number of bins of the FFT e.g., 1024, 2048 given by $2^{k}$ where " $k$ " is an integer. The number of frequency bins in the FFT is determined by processing constraints and the capability of available hardware.

The FFT noise floor adds to the SNR of the ADC resulting in a total noise floor of SNR + FFTN. Therefore it is crucial to use the required number of samples in the FFT to distinguish the harmonic distortion components from the noise floor and reduce the effect of processing gain of the FFT [1]. The total noise floor [46] is then

\footnotetext{
${ }^{59}$ Assuming each code has the same equal probability of appearing in the conversion. This would be consistent with a linear ramp input.

${ }^{60}$ Differential signal.
} 
given by

$$
N F l_{d B}=-6.02 \times N-10 \cdot \log _{10}(M)+1.25,
$$

where " $N$ " is the number of bits of the ADC.

The dynamic performance metrics were obtained from the power spectrum of the FFT as presented in [46]. The PSD of the noise floor taking into account both quantization noise and analog noise is given by

$$
|N F l|^{2}=\left.\frac{\sum_{k=1, k \neq J, k \neq h J}^{M / 2-1}|Y(k)|^{2}+\frac{1}{2}\left|Y\left(\frac{M}{2}\right)\right|^{2}}{M / 2-h_{\max }}\right|_{h=2 \ldots h_{\max }},
$$

where $f_{i}=J \frac{f_{s}}{M}$ and the spectrum ranges from 0 to $M / 2$ ignoring the imaginary components of the FFT. The noise floor is then the sum of the components in the spectrum "Y" excluding the DC component and the harmonics ${ }^{61}[46]$.

The SINAD is the ratio of the input signal to both noise and the harmonic distortion spectral components [46]. The SINAD is a strong function of the SNR and the two metrics typically track together as a function of the amplitude of the input signal [47]. The SINAD can be found with

$$
S I N A D_{d B}=10 \cdot \log _{10} \frac{|Y(J)|^{2}-|N F|^{2}}{\sum_{k=1, k \neq J}^{M / 2-1}|Y(k)|^{2}+2|N F|^{2}+\frac{1}{2}\left|Y\left(\frac{M}{2}\right)\right|^{2}}
$$

The ENOB is a metric which indicates the resolution of the ADC (in bits) for which it operates as an ideal ADC and can be computed from the SINAD [1] with

$$
E N O B=\frac{S I N A D-1.76}{6.02}
$$

\footnotetext{
${ }^{61}$ Typically it is sufficient to remove the first 7 harmonics.
} 
and is typically calculated with the SINAD at full scale input. The SNR is the typical signal to noise ratio

$$
S N R_{d B}=\left.10 \cdot \log _{10} \frac{|Y(J)|^{2}-|N F l|^{2}}{\sum_{k=1, k \neq J, k \neq h J}^{M / 2-1}|Y(k)|^{2}+\left(h_{\max }+1\right)|N F l|^{2}+\frac{1}{2}\left|Y\left(\frac{M}{2}\right)\right|^{2}}\right|_{h=2 \ldots h_{\max }} .
$$

It excludes harmonic components past the fundamental as well as correcting for the noise contributed by the noise floor to the harmonic components [46]. The total harmonic distortion (THD),

$$
T H D_{d B}=10 \cdot \log _{10} \frac{\sum_{h=2}^{h_{\max }}|Y(h J)|^{2}}{|Y(J)|^{2}}
$$

is the ratio of the sum of all rms harmonic components to the rms value of the fundamental as shown in Eq. 5.8. The SFDR,

$$
S F D R_{d B}=\left.10 \cdot \log _{10} \frac{|Y(J)|^{2}}{\max |Y(h J)|^{2}}\right|_{h=2 \ldots h_{\max }}
$$

is the spurious free dynamic range is the ratio between the amplitude of the fundamental and the amplitude of the next highest harmonic spur. The equations presented above were paired with a modified MATLAB template presented in [48] to analyze the data presented in this chapter.

An example of the FFT spectrum is presented in Fig. 5.6 and its time domain source in Fig. 5.7. In order to satisfy the requirements for coherent sampling the frequency of the input sinusoid was $135.5 \mathrm{kHz}$ for a sampling frequency of $19.968 \mathrm{MHz}$. For an FFT resolution of 1024 bins, 7 input cycles were observed in $51.282 \mu s$. 


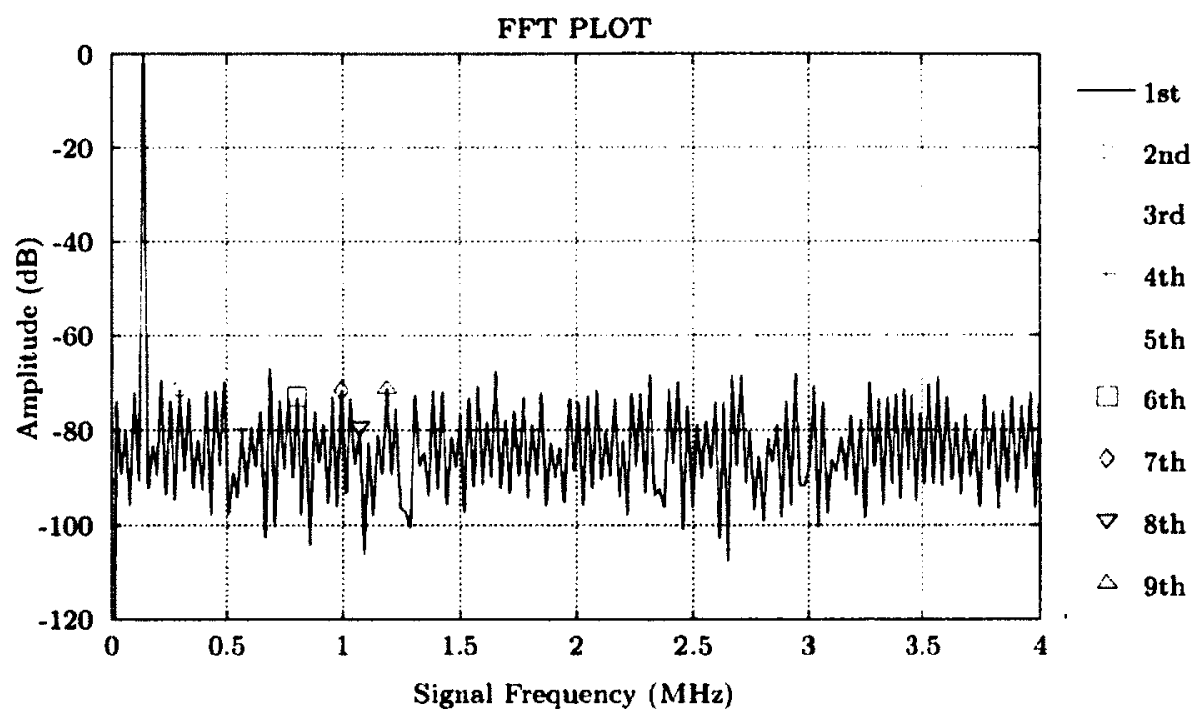

Figure 5.6: FFT spectrum of the input signal, $f_{s}=19.9680 \mathrm{MHz}, f_{i}=0.1365 \mathrm{MHz}$, $\mathrm{n}=7, \quad \mathrm{~m}=1024, \quad \mathrm{ENOB}=7.916, \quad \mathrm{SINAD}=49.491 \mathrm{~dB}, \quad \mathrm{SFDR}=68.513 \mathrm{~dB}$ $\mathrm{THD}=64.280 \mathrm{~dB}$.

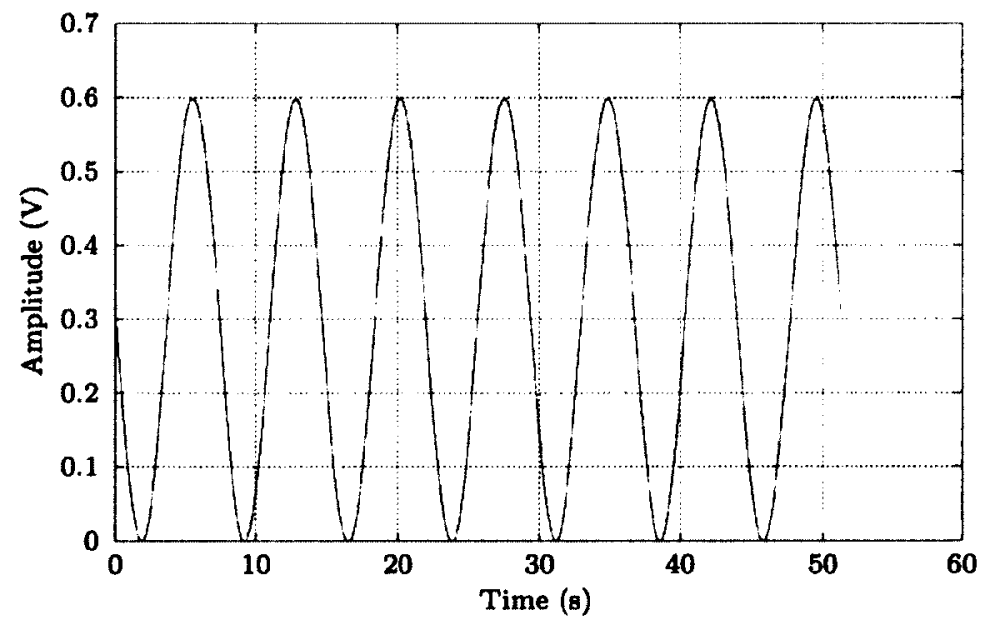

Figure 5.7: Reconstructed analog output using ideal DAC, $f_{s}=19.9680 \mathrm{MHz}$, $\mathrm{f}_{\mathrm{i}}=0.1365 \mathrm{MHz}, \quad \mathrm{n}=7, \mathrm{~m}=1024, \quad \mathrm{ENOB}=7.916, \quad$ SINAD $=49.491 \mathrm{~dB}$, $\mathrm{THD}=64.280 \mathrm{~dB}$.

\subsubsection{Jitter Analysis of Dynamic Latched Comparator}

To measure the noise floor of the ADC it is expedient to start by measuring the noise contributed by any given stage. Since the output of a comparator is a digital 
logic value it is difficult to use traditional noise measurement techniques ${ }^{62}$. Noise originating from the comparator's devices is translated into an uncertainty on the sampling time of the comparator, jitter. All simulations for this ADC were performed with an ideal SAH. Removing the SAH introduces further uncertainty into sampling time causing the performance of the $\mathrm{ADC}$ to reduce as the input frequency approaches the sampling frequency of the ADC.

Using PSS and PNOISE analysis with Cadence Tools the jitter of the dynamic latched comparator was measured for a full scale and low level input, the results of which were in turn averaged. In [1], the SNR of the ADC in $\mathrm{dB}$ for a full scale input,

$$
S N R_{d B F S}=-20 \log \left(2 \pi f_{s} t_{j}\right)
$$

is used to convert the jitter, $t_{j}$ which is in units of "rms s" to a theoretical limit on the SNR at a clock frequency $f_{\mathrm{g}}$. The jitter was simulated for only one stage, as PSS and PNOISE jitter analysis does not converge for large numbers of devices. In the calculation it was assumed that each stage contributes approximately the same amount of jitter, or noise to the overall conversion noise floor. The jitter measurements were performed at a Nyquist frequency ${ }^{63}$ at near Nqyuist rates. The average of the worst case jitter was measured to be $t_{j}=482 \mathrm{fs}$. The theoretical noise floor of the $\mathrm{ADC}$ based on the sampling uncertainty of the comparators is then $-66.293 \mathrm{~dB}$ relative to the fundamental.

\footnotetext{
${ }^{62}$ Using transient analysis with noise requires a large amounts of data points and post processing. ${ }^{63}$ The actual frequency was $f_{s}-\Delta f_{w}$ where $\Delta f_{w}=100 \mathrm{kHz}$.
} 


\subsubsection{Dynamic Analysis Metrics}

The SNDR and the SFDR for the ADC is presented in Fig. 5.8. For a constant sampling frequency of $f_{s}=20 \mathrm{MHz}$. Dynamic testing was performed with a full scale sinusoidal input of $300 \mathrm{mV}$ single ended peak-to-peak corresponding to a differential waveform of $600 \mathrm{mV}$ differential peak-to-peak. The input frequency $f_{i}$ was swept from a low input frequency of $136.5 \mathrm{kHz}$ up to $40 \mathrm{MHz}$ to find the ERBW of the ADC. All simulations were performed with an ideal sample-and-hold (SAH) VHDL component at the front end of the ADC. Without the use of a SAH circuit, the dynamic performance of the ADC would degrade as the input frequency approaches the sampling rate. Operating without a SAH implies that the input waveform is changing during the conversion process. This introduces jitter, as the location of the sampled point can vary significantly. The jitter appears as noise in the frequency spectrum lowering both the SINAD and the SFDR of the converter.

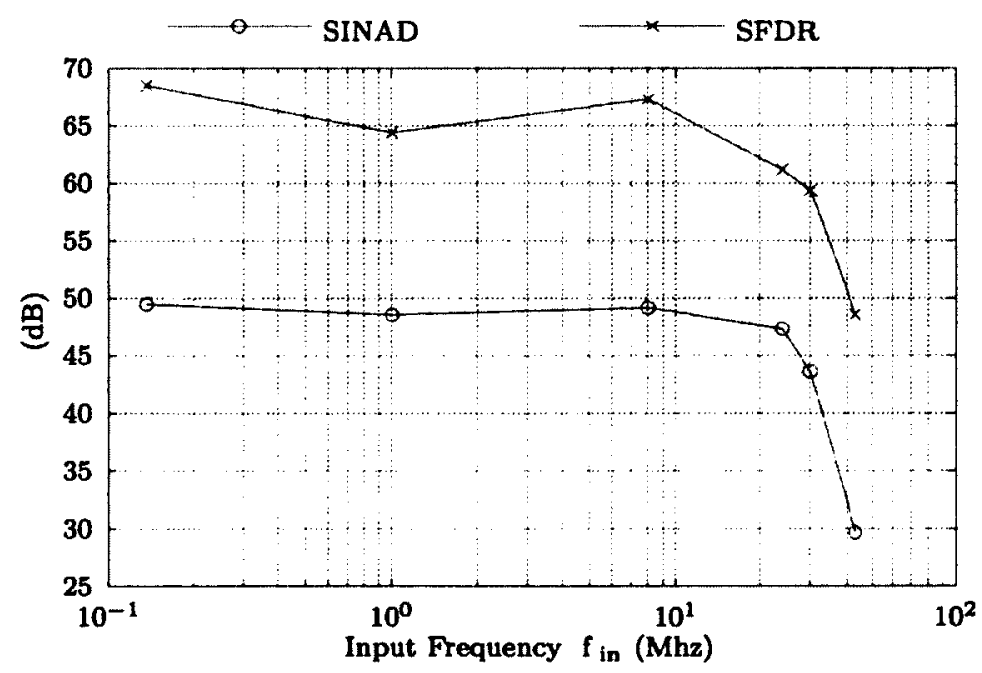

Figure 5.8: SINAD and SFDR for a sampling frequency $f_{s}=20 \mathrm{MHz}$ as a function of the input frequency $f_{\text {in }}$

The point at which the SINAD drops by $3 \mathrm{~dB}$ below its full scale value is the effective resolution bandwidth or ERBW. It corresponds to an uncertainty greater than 
LSB/2 for the digital word. In the CABS architecture this point is determined mainly by the conversion time through the cascaded chain of comparators. At this point the comparators in the final stage of the ADC (responsible for the LSB conversion) miss the conversion altogether for one or more $\operatorname{codes}^{64}$. For clarity the effective number of bits (ENOB) is presented separately in Fig. 5.9.

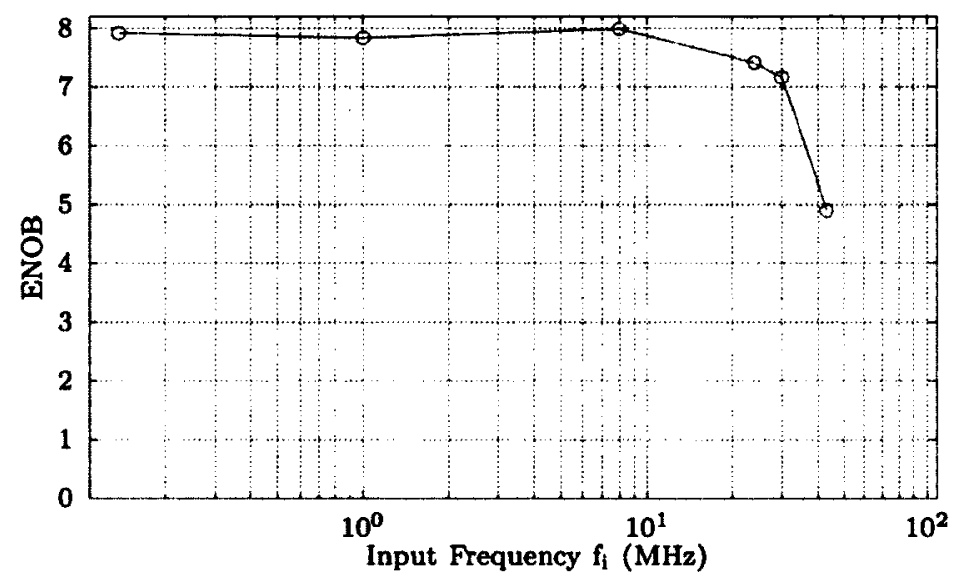

Figure 5.9: ENOB for a sampling frequency $f_{s}=20 \mathrm{MHz}$ as a function of the input frequency $f_{\text {in }}$.

\footnotetext{
${ }^{64}$ As the rising edge of the clock signal from the b1 stage triggers the b0 converter it does not rise above $\mathrm{V}_{\text {thp }}$ in time as it's asynchronously reset by the falling edge of the global clock signal.
} 


\subsection{Power Dissipation}

The power consumption was simulated with a full scale input as was performed in Sec. 5.2.2. For power consumption the input frequency was kept at $987 \mathrm{kHz}$ while sweeping the sampling rate. A larger input frequency was used to reduce simulation time for nine full input cycles. The figure of merit used to characterize the performance of the ADC is given by

$$
E=\frac{P_{s w}}{2^{E N O B} f_{s}},
$$

which is the same metric used in [14].

The power consumption of the ADC core is presented in Fig. 5.10. The measured ADC core power consumption does not include any input or output buffers of the ADC. The power consumption of the full ADC is presented in Fig. 5.11. The total power consumption includes all the elements on the IC.

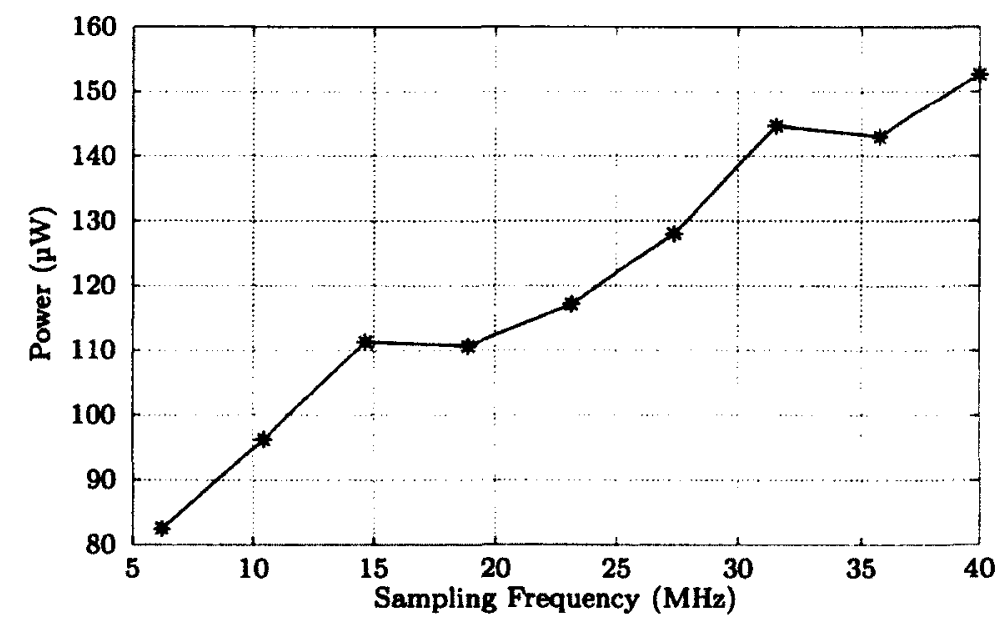

Figure 5.10: Power dissipation of the digital core as a function of the sampling frequency $f_{s}$ for an input frequency of $f_{i}=0.987 \mathrm{MHz}$. 


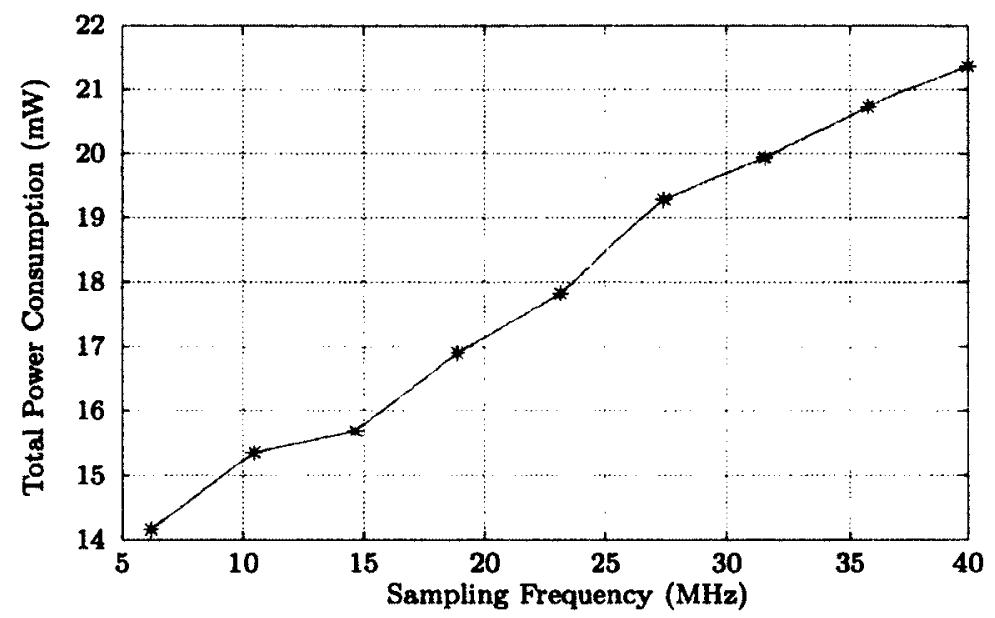

Figure 5.11: Power dissipation of the full IC, as a function of the sampling frequency $f_{s}$ for an input frequency of $f_{i}=0.987 \mathrm{MHz}$.

The FOM in terms of the ADC core power consumption is presented in Fig. 5.12. At a sampling rate of $25 \mathrm{MHz}$ the ADC achieves a similar FOM, $15.8 \mathrm{fJ}$, as the work presented in [14] which reports an FOM of $15 \mathrm{fJ}$. In comparison to [14], approximately the same FOM is obtained for an ADC with 8 bits as opposed to 6 bits and a larger input range ${ }^{65}$. Additionally this work was implemented in a $130 \mathrm{~nm}$ technology as opposed to [14] which is implemented in a superior $90 \mathrm{~nm}$ process.

\footnotetext{
${ }^{65}$ The input range is twice as large, increasing dynamic range but reducing the operating frequency.
} 


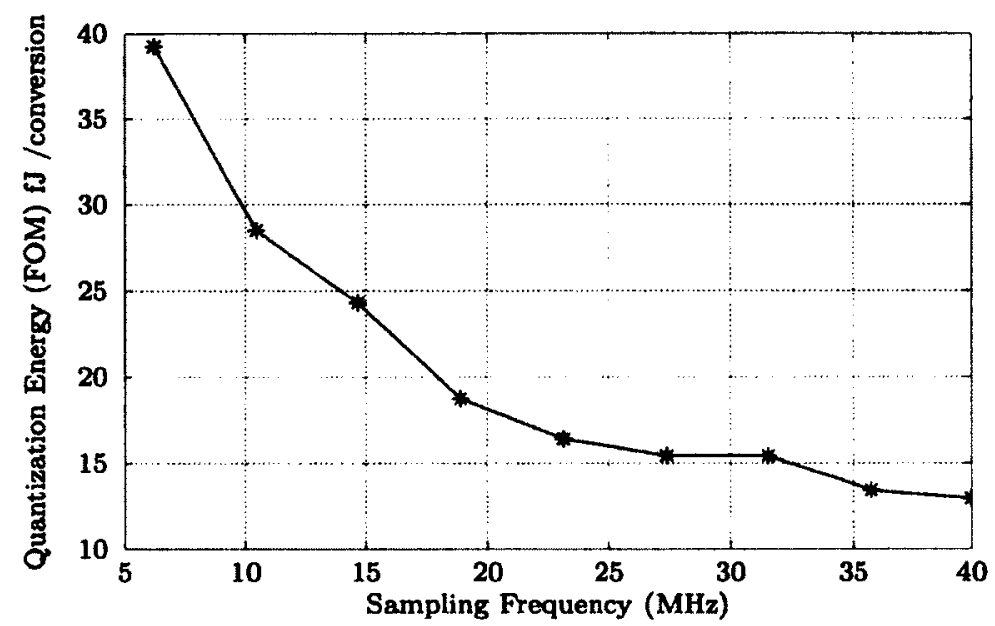

Figure 5.12: FOM for the core power consumption, as a function of the sampling frequency $f_{s}$ for an input frequency of $f_{i}=0.987 \mathrm{MHz}$.

\subsection{The Effects of ADC Design Choices on Static and Dynamic Performance Metrics}

In terms of static analysis, the INL and DNL are determined mainly by the capability of the tuning strategy to bring the thresholds of the comparators within LSB/4. In the interest of maintaining LSB/4 minimum tuning resolution on the thresholds generated by the SCMTA devices, the smallest unit sized device must be made as small as possible. This in turn limits the available tuning range which reduces the linear input range of the ADC since the SCMTAs can no longer adjust for both desired thresholds and PVT within the specified accuracy unless more tuning bits are added to the coarse binary array. This in turn increases the conversion delay and reduces the operating frequency.

As the resolution of the ADC grows, the voltage range corresponding to $1 \mathrm{LSB}$ shrinks for the same differential ADC input range. Eventually, even using the minimum sized CMOS device for the nominal SCMTA device available in the technology node, the noise floor becomes bigger than LSB/4 of the ADC. As well, the linear 
input range cannot be arbitrarily expanded as was discussed in Sec. 4.1.3 because IDWI of the devices. The resolution of the CABS ADC can be said to suffer from the same fundamental limitation as the Flash ADC. Without the use of some pipelining techniques it is doubtful whether the CABS ADC can be pushed past the typical maximum resolution of a Flash ADC [1]. This happens to be in the range of 10 to 12 bits. Each additional bit, increases the noise floor and the conversion time. It would be conceivable using power hungry techniques to correct for offset and improve the slew rate of the comparators pushing the resolution of the CABS ADC further. A smaller technology node such as $65 \mathrm{~nm}$ could achieve relatively smaller capacitive gradations. Since the major design goal of the CABS architecture is low energy per conversion this would be undesirable.

For dynamic analysis there are a large number of metrics, of which only the most fundamental are discussed. The linear input range of the ADC directly influences the dynamic range of the ADC. Lowering the input range or increasing the resolution of the $\mathrm{ADC}^{66}$ causes the noise floor of the $\mathrm{ADC}$ to rise relative to the input signal. This decreases the dynamic metrics of the ADC such as SINAD, SNR, THD. Since the ENOB is typically calculated from the SINAD, this metric decreases as well.

The frequency of operation of the ADC is determined by the total conversion time through the highest capacitive imbalance and the lowest voltage input paths. The delay through the highest capacitive imbalance path can be reduced by decreasing the ratio of the differential inputs at the expense of the linear input range. The smallest input voltage path can be improved by switching to a newer technology node ${ }^{67}$ or using larger device sizes in the comparator. The second method has immediate trade offs with the linear input range as well as requiring larger SCMTA devices for the same input range which in turn add the to the conversion delay resulting in a marginal

\footnotetext{
${ }^{66}$ For the same differential input range.

${ }^{67}$ This would be the preferred method, devices have a higher $f_{t}$ and $g_{m}$.
} 
improvement.

The power consumption of CABS ADCs is on the order of a hundred $\mu \mathrm{W}$ resulting in an excellent FOM, as they can operate at higher frequencies than their SAR counterparts. The power consumption can be further improved by switching to a deeper sub micron technology node. Although the structure of the CABS ADC is elegant in its simplicity, the optimization on a circuit design level becomes challenging when trying to achieve the desired system level performance metrics since the majority of ADC elements must be designed concurrently. 


\subsection{Summary of Simulation Results}

A complete summary of the static and dynamic performance metrics of the ADC is presented in Tb. 5.1. The ASIC was implemented in a IBM CMRF8SF $130 \mathrm{~nm}$ technology for a power supply of $1.2 \mathrm{~V}$. The input range was $600 \mathrm{mV}$ peak-to-peak differential. The maximum sampling frequency to guarantee conversion code integrity was $25 \mathrm{MHz}$. The ENOB at DC for the ADC was 7.98 with a SINAD of $49.1 \mathrm{~dB}$ and a SFDR of $66.3 \mathrm{~dB}$. The ADC achieved an excellent power consumption for the core in terms of the FOM of $15.8 \mathrm{fJ}$ per conversion. The work presented in [14] required 48 tuned comparators and half the input range resulting in a smaller layout area.

Table 5.1: Summary of simulation results.

\begin{tabular}{cc}
\hline Metric & Simulation Value \\
\hline Technology & IBM CMRF8SF 130 nm \\
VDD & $1.2 \mathrm{~V}$ \\
Input Range & $600 \mathrm{mV}_{\text {pk-pk,diff }}$ \\
Sample Rate & $20 \mathrm{MHz}$ \\
ERBW & $25 \mathrm{MHz}$ \\
Area & $4.064 \mathrm{~mm}^{2}$ \\
INL & $0.483 \mathrm{LSB}$ \\
DNL & $0.432 \mathrm{LSB}$ \\
SINAD @ Nyquist & $49.1 \mathrm{~dB}$ \\
SFDR @ Nyquist & $66.3 \mathrm{~dB}$ \\
ENOB @ DC & 7.98 \\
Power & $122 \mu \mathrm{W}$ \\
FOM & $15.8 \mathrm{fJ}$ \\
\hline
\end{tabular}

A comparison of the relevant low power architectures in recent publications was presented in Sec. 2.1.6. The same figures and tables are reproduced along with the simulation results of the CABS ADC presented in this chapter. Since frequency and resolution are a typical design trade off, the CABS ADC presented in this work 
achieves moderate resolution but with excellent FOM, as is pictured in the bottom left side of Fig. 5.13. To achieve this improvement of two bits over [14] the frequency of operation drops to the bottom left corner of Fig. 5.14. In Tb. 5.2 all the ADC operating parameters of low power architectures with similar performance are compared to the simulation results presented in this chapter.

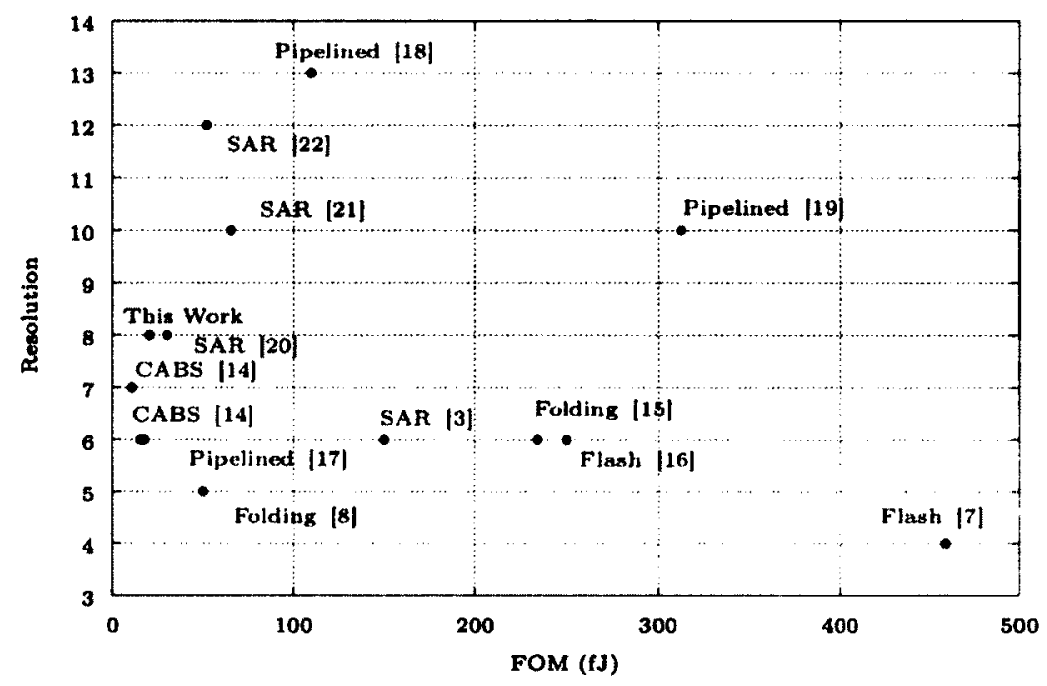

Figure 5.13: Comparison of $A D C$ resolution as a function of the figure of merit for published ADCs compared to CABS ADC simulation results.

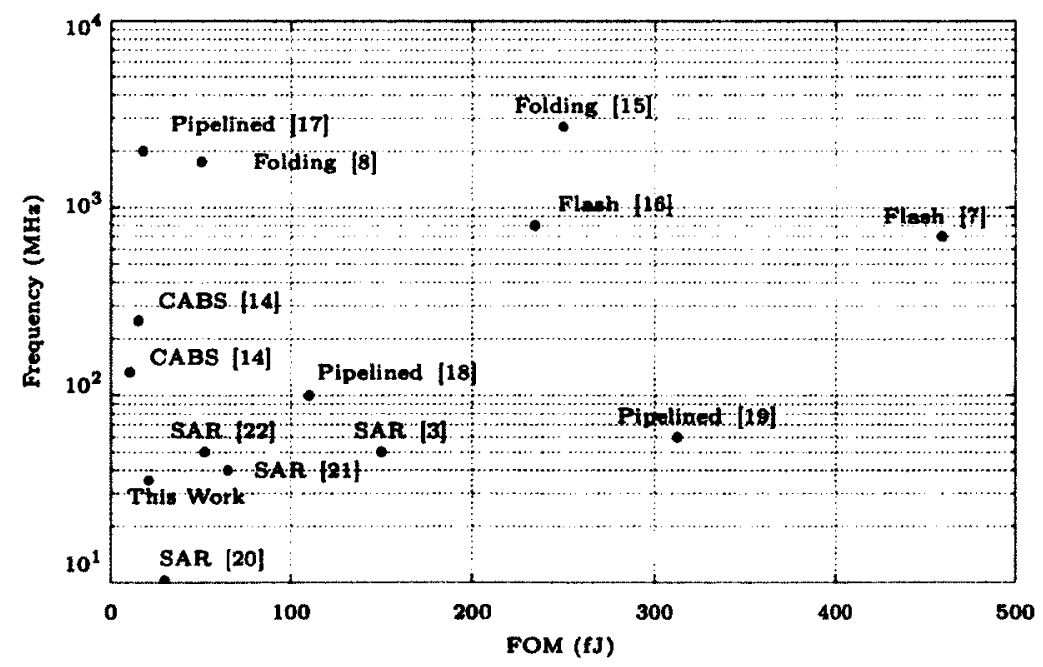

Figure 5.14: Comparison of the frequency of operation as a function of the figure of merit for published ADCs compared to CABS ADC simulation results. 
Table 5.2: Comparison of simulated results to published state-of-the art low power CMOS based ADC designs.

\begin{tabular}{|c|c|c|c|c|c|c|c|c|c|}
\hline Architecture & VDD & Technology & Area $\left(\mathrm{mm}^{2}\right)$ & Power (mW) & Speed (MS/s) & Input Range (mV,diff) & Resolution & ENOB & FOM \\
\hline This Work & $1.2 \mathrm{~V}$ & $0.13 \mu \mathrm{m}$ & 4 & 0.122 & 25 & 600 & 8 & 7.98 & 16.8 \\
\hline CABS [14] & $1 \mathrm{~V}$ & $90 \mathrm{~nm}$ & 0.050 & 0.140 & 250 & 384 & 6 & 5.3 & 15.0 \\
\hline CABS [14] & $1 \mathrm{~V}$ & $90 \mathrm{~nm}$ & 0.055 & 0.133 & 133 & 768 & 7 & 6.4 & 10.4 \\
\hline Folding [8] & $1 \mathrm{~V}$ & $90 \mathrm{~nm}$ & 0.017 & 2.200 & 1750 & 800 & 5 & 4.7 & 50.0 \\
\hline Folding [15] & $1 \mathrm{~V}$ & $90 \mathrm{~nm}$ & 0.360 & 50.000 & 2700 & 800 & 6 & NS & 250.0 \\
\hline Flash [7] & $1.8 \mathrm{~V}$ & $0.18 \mu \mathrm{m}$ & NS & 4.430 & 700 & NS & 4 & 3.8 & 460.0 \\
\hline Flash [16] & $1.2 \mathrm{~V}$ & $60 \mathrm{~nm}$ & 0.130 & 12.000 & 800 & NS & 6 & 5.6 & 234.0 \\
\hline Pipelined [17] & $1.1 \mathrm{~V}$ & $40 \mathrm{~nm}$ & 0.030 & 2.300 & 2000 & 640 & 6 & 5.5 & 17.4 \\
\hline Pipelined [18] & $1.3 \mathrm{~V}$ & $0.13 \mu \mathrm{m}$ & 1.200 & 91.000 & 100 & 2000 & 13 & NS & 110.0 \\
\hline Pipelined [19] & $1.2 \mathrm{~V}$ & $0.13 \mu \mathrm{m}$ & 0.980 & 19.200 & 60 & NS & 10 & NS & 313.0 \\
\hline SAR [3] & $1 \mathrm{~V}$ & $90 \mathrm{~nm}$ & 0.055 & 0.240 & 50 & 320 & 6 & 5.0 & 150.0 \\
\hline SAR [20] & $1 \mathrm{~V}$ & $90 \mathrm{~nm}$ & 0.132 & 0.069 & 10 & 400 & 8 & 7.8 & 30.0 \\
\hline SAR [21] & $1 \mathrm{~V}$ & $65 \mathrm{~nm}$ & 0.060 & 1.210 & 40 & 1000 & 10 & 8.9 & 65.0 \\
\hline SAR [22] & $1.3 \mathrm{~V}$ & $90 \mathrm{~nm}$ & 0.160 & 3.600 & 50 & 2000 & 12 & 10.6 & 52.0 \\
\hline
\end{tabular}




\section{Chapter 6}

\section{ADC Measured Results}

The 8-bit CABS ADC was fabricated through the Canadian Microelectronics Corporation (CMC). A photograph of the fabricated IC is presented in Fig. 6.1. The die in the photograph was bonded through a CMC post fabrication service using a CQFP44A high quality $\mathrm{RF}$ compatible ceramic package manufactured by Kyocera and is presented in Fig. 6.2. This chapter presents the measured performance of the fabricated CABS ADC also referred to as "ICGCUOCT" along with its testing methods.

The test configurations used to extract ADC performance metrics are discussed in Sec. 6.1. The design of two printed circuits boards (PCBs) along with appropriate PCB design practices is presented in Sec. 6.2. The results of preliminary ADC testing which led to the design of the second PCB are presented in Sec. 6.3. A calibration strategy similar to the one proposed in [14] to set threshold levels and correct for PVT variations is illustrated in Sec. 6.4. Finally, the chapter concludes with a discussion of power dissipation in Sec. 6.7 and a summary of all measurement results in Sec. 6.7. 


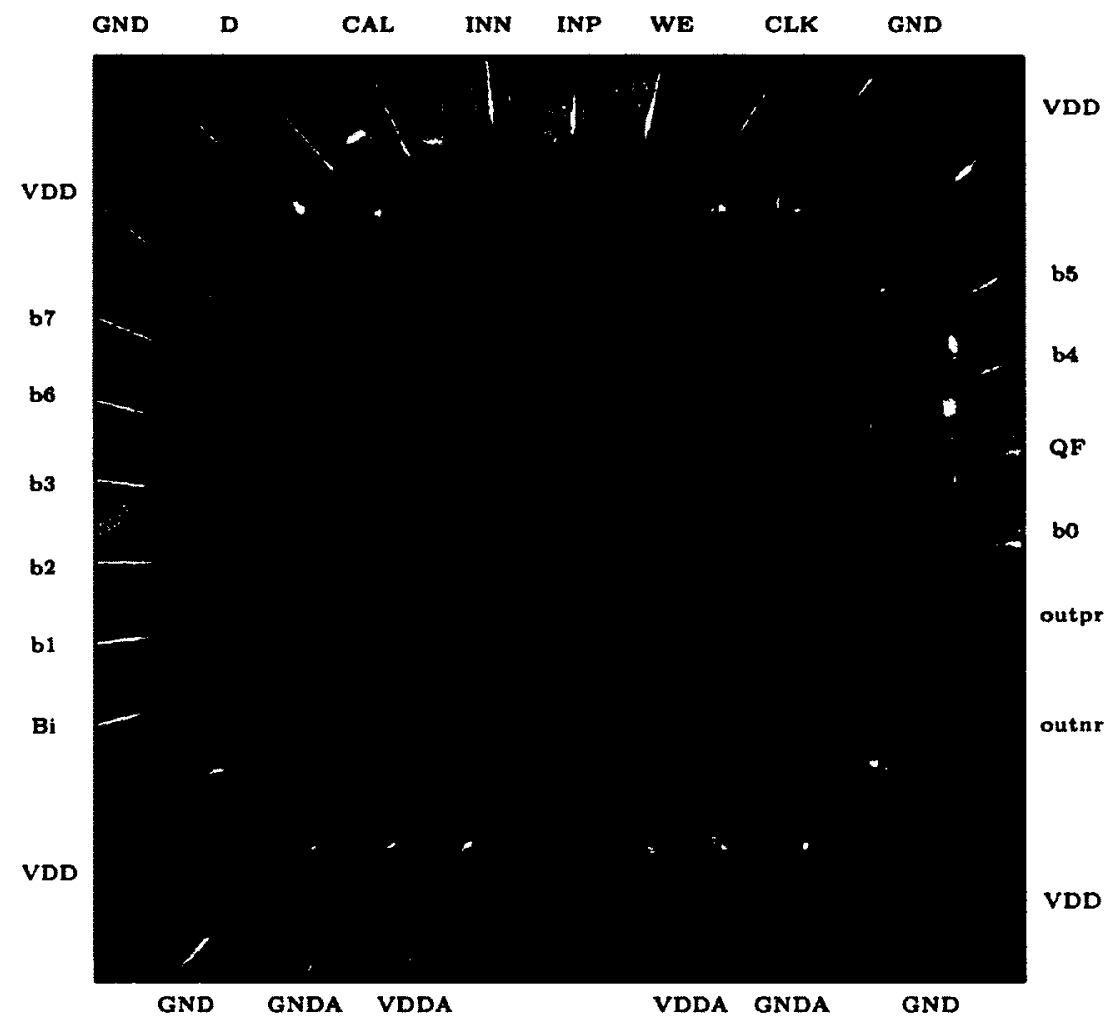

Figure 6.1: Photograph of the fabricated IC. The bond wires are labeled instead of the pads to improve visibility. The IC was bonded by CMC and uses a CQFP44A ceramic package.

\subsection{Test Configuration}

For the test bench, several configurations were used depending on the $\mathrm{PCB}$ version and the kind of $\mathrm{ADC}$ test, specifically, the type of ADC test; dynamic $\mathrm{AC}$ or static $\mathrm{DC}$ test. Some of the equipment common to all test configurations is discussed in Tb. 6.1. A computer was used as a test controller with Matlab as the choice of development environment.

A "Keithley 2600" source meter was used to power the PCB and monitor the power consumption. The source meter is connected to the computer via an RS-232 serial link. The ADC "CLK", "INP", and "IN" signals are generated using the "Agilent $81150 A^{\prime \prime}$ arbitrary signal generator. The shift register control signals are generated 


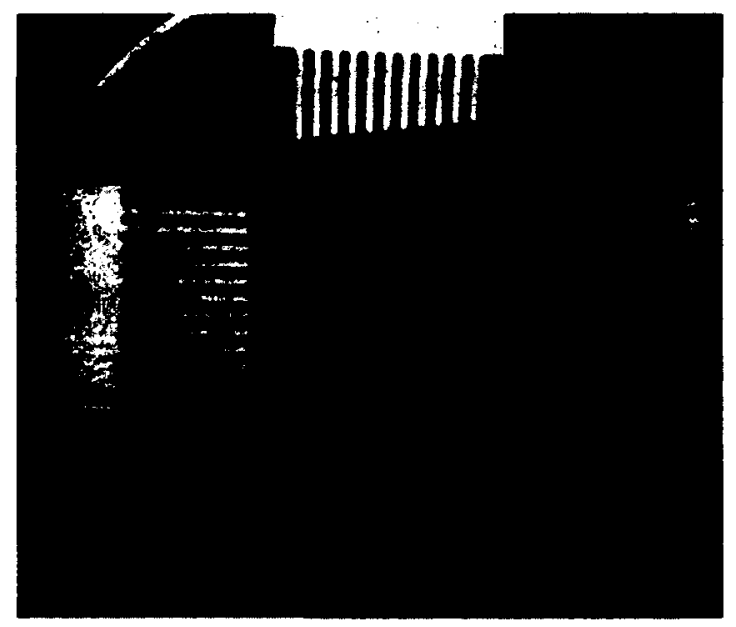

Figure 6.2: Photograph of the packaged IC in the CQFP44A ceramic package.

from two "Agilent U2331A" NI-DAQs. "Agilent U2331A" NI-DAQs are connected via USB to the computer and are used to generate the shift register signals at a frequency of $500 \mathrm{kHz}$. Finally the output of the ADC is connected via an IDE header to the digital sampling oscilloscope ("Agilent 5855A"). The scope is in turn connected to the computer via an ethernet link. A summary of the components used is presented in Tb. 6.1.

Table 6.1: Test bench equipment.

\begin{tabular}{lll}
\hline Component & Function & Signals \\
\hline Keithley 2600 & Source meter and 1.2V power supply for the PCB. & VDD \\
Agilent U2331A & NI-DAQ / Data acquision / Signal generation & WE, D, CAL \\
Agilent 81150A & Arbitrary function generator & INP, INN, CLK \\
Agilent 33205A & Optional CLK or DAC CLK & \\
Agilent 54832DSO & Digital spectrum oscilloscope & b0-b7
\end{tabular}

\subsubsection{Test Configuration 1}

A functional diagram of the preliminary test bench for the ADC can be found in Fig. 6.3. This test bench was used for the first PCB. The arbitrary signal generator 
is capable of generating differential signals such as a slow ramp, staircase pattern, DC levels, as well as the standard sinusoidal, square wave and pulse shape. With the $81150 \mathrm{~A}$ it is then possible to test both $\mathrm{AC}$ and $\mathrm{DC}$ performance metrics.

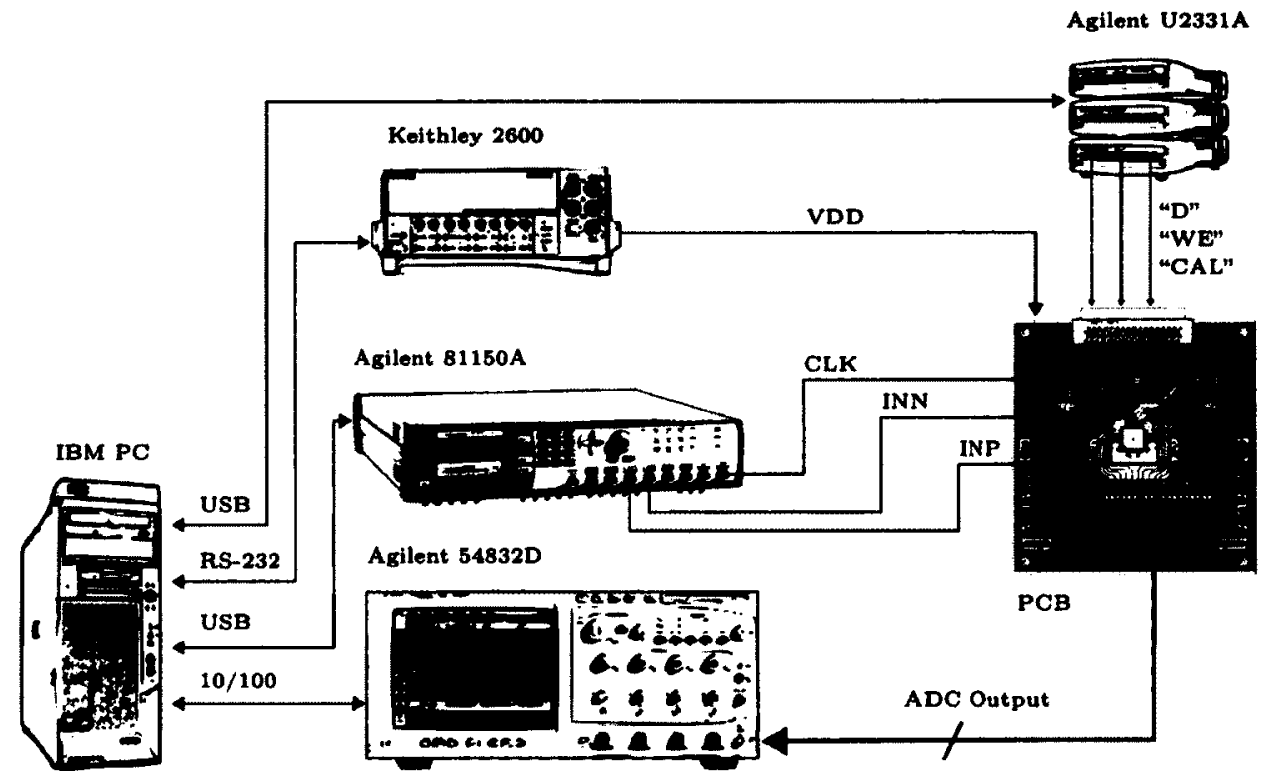

Figure 6.3: Functional diagram for preliminary test plan.

The test bench was then modified to use a DAC for back-to-back testing of the ADC. The DAC is the 12-bit "DAC5652" on the evaluation board "DAC5652EVM" manufactured by Texas Instruments. When using a back-to-back configuration it is necessary for the DAC to have a minimum of 2 bits higher resolution than the ADC [1].

\subsubsection{Test Configuration 2}

The main test configuration used for the second PCB is presented in Fig. 6.5. The most noticable change is the replacement of the "Agilent U2331A" with a serial parallel interface bus (SPI) interface to control the shift register inputs. This was done 


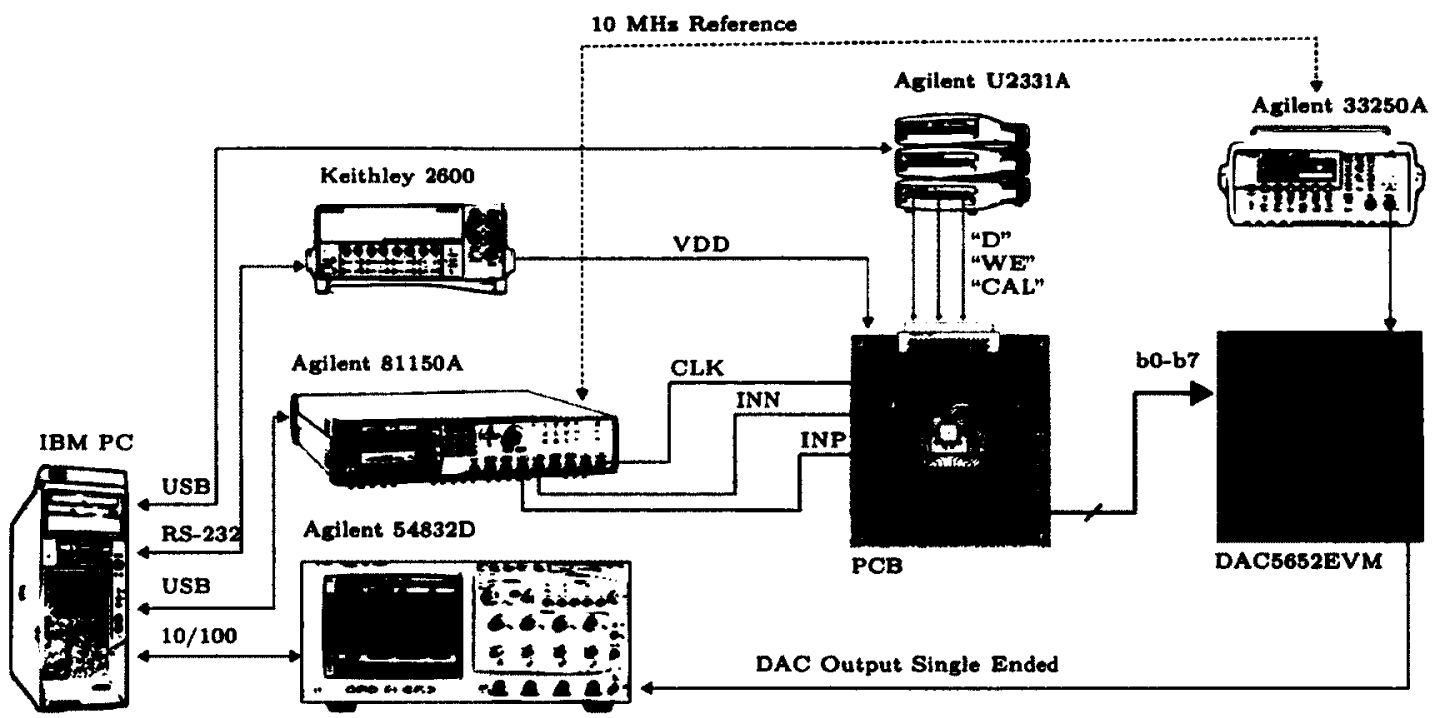

Figure 6.4: Functional diagram for preliminary test plan with back-to-back configuration.

to improve the certainty with which the calibration code is loaded into the shift register. In addition three "Keithely 2600 " power supply and source meters are used to generate the $1.2 \mathrm{~V}, 3.3 \mathrm{~V},-/+5 \mathrm{~V}$ and $0.6 \mathrm{~V}$ supply levels necessary to power additional circuitry needed for the second PCB. The board was also connected in the second configuration similar to the functional diagram presented in Fig. 6.4. The presence of level shifting translators on the second PCB allowed for direct coupling of PCB2 to the "DAC5652EVM" which requires TTL logic level inputs. Previously, this had been achieved by placing the logic translator on a prototyping board and connecting the ADC and the DAC to the translator via spliced IDE cables. 


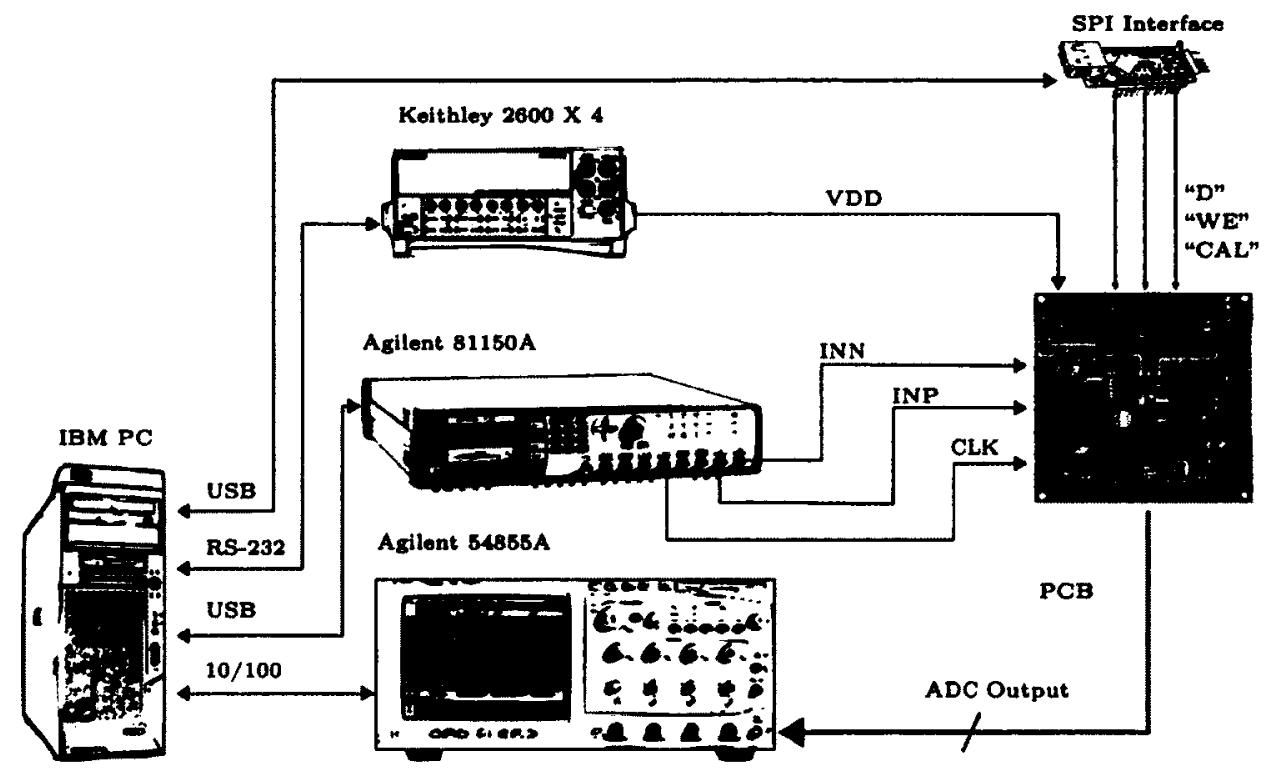

Figure 6.5: Functional diagram for PCB2 test plan.

\subsection{PCB Design}

Two printed circuit boards were designed and manufactured to accommodate the testing process of the CABS ADC. The first PCB is discussed in Sec. 6.2.3. After an initial testing phase it became evident that additional components, such as an external SAH, are necessary to improve the functionality of the ADC as is discussed in Sec. 6.3. The design of the PCB with external SAH ICs, "PCB2", is discussed in Sec. 6.2.4.

\subsubsection{Power Supply Decoupling}

The ADC presented in this work along with any ICs discussed in the proceeding section are sensitive to ripples on the power supply traces. For the ADC, ripples on the supply lines are translated into jitter (noise) degrading the overall performance of the ADC [1]. This phenomenon becomes more prevalent with an increasing frequency of operation. On both the PCBs designed, power supply decoupling was achieved by 
a combination of decoupling capacitors and ferrite beads. The types of decoupling capacitors and their magnitudes is presented in Tb. 6.2.

Table 6.2: Decoupling component device sizes.

\begin{tabular}{llll} 
Device Value & Manufacturer & Manufacturer ID & Description \\
\hline $10 \mathrm{uF}$ & Nichicon & TCM0J106M8R & CAP TANT 10UF 6.3V 20\% 0603 \\
\hline $0.1 \mathrm{uF}$ & Kemet & C0603C104K8RACTU & CAP CER 0.1UF 10V 10\% X7R 0603 \\
\hline $27 \mathrm{pF}$ & Murata & GQM2195C1H270JB01D & CAP CER 27PF 50V 5\% NP0 0603 \\
\hline $1 \mathrm{~K} \Omega @ 100 \mathrm{MHz}$ & TDK & MMZ1608Y102B & FERRITE CHIP 1000 OHM 400MA 0603
\end{tabular}

Tantalum capacitors on the order of $10 \mu \mathrm{F}^{68}$ are used to ground low frequency transient noise. Their main role is to serve as a charge reservoir [1] so that the power supplied to the IC does not depend on the length of the supply trace (inductance). Smaller high quality X7R ceramic capacitors are connected closer to the power pins of an IC. This is done to ground high frequency noise near the IC. The decoupling capacitors are then grounded through a large ground plane present on both sides of the PCB reducing any inductance in the return path. As well, a ferrite bead is used to block high frequency noise and isolate ICs from the power mains. Ground connections for all ICs on the PCBs are made via contacts straight to the ground planes. This reduces the inductances through a possible ground loop.

\subsubsection{Signal Integrity and Propagation}

Signal traces have a maximum allowable electrical length before impedance matching becomes necessary to avoid reflections on the lines. The maximum allowable electrical length [49] is

$$
L_{\text {trace }}=\frac{R T}{2 t_{P D}}
$$

\footnotetext{
${ }^{68} \mathrm{High}$ quality electrolytic capacitors could also be used.
} 
where "RT" is one half of the one way propagation delay and " $t_{P D}$ " is the built in propagation delay. The built in propagation delay [49] is given by,

$$
t_{P D}=84.75 \sqrt{0.475 \varepsilon_{r}+0.67} \mathrm{ps} / \text { in } .
$$

For a dielectric constant of 4.7 in FR4 material, the built in propagation delay is $144.4 \mathrm{ps} / \mathrm{in}$. The propagation delay for the output drivers of the ADC assuming a $15 \mathrm{pF}$ load with $1.5 \mathrm{nH}$ of line inductance is around $3 \mathrm{~ns}$. This gives a maximum length of 41 inches. Instead if we consider the traces as carrying analog signals then a typical benchmark is for the maximum trace length [49] to be less than

$$
L_{\text {trace }}<\frac{\lambda}{20} \text {. }
$$

Using the value in [42] for the propagation delay in FR4 ${ }^{69}$ type material on an outer trace, the propagation speed in FR4 is $5.5 \mathrm{in} / \mathrm{ns}$. At a typical ADC operating frequency of $30 \mathrm{MHz}$ the maximum allowable length is $9.3 \mathrm{in}$.

Signal integrity is improved through the appropriate grounding of signal return paths. Since both the top side and the back side of the PCBs have ground planes, at any given time signal traces have nearby connection to the ground plane for the returns. To reduce any possible ground loops, vias between the two ground planes cover the entire PCB, as is presented in the example Fig. 6.6.

\subsubsection{Preliminary PCB Design, "PCB1"}

The first PCB was designed using CADSOFT Eagle Tools Pro Version 5.1. A photograph of the top side of the PCB is presented in Fig. 6.7. The packaged IC is at the

\footnotetext{
${ }^{69}$ The PCB is made from a woven fiber glass composite material FR4. The electrical properties can vary slightly between manufacturers.
} 


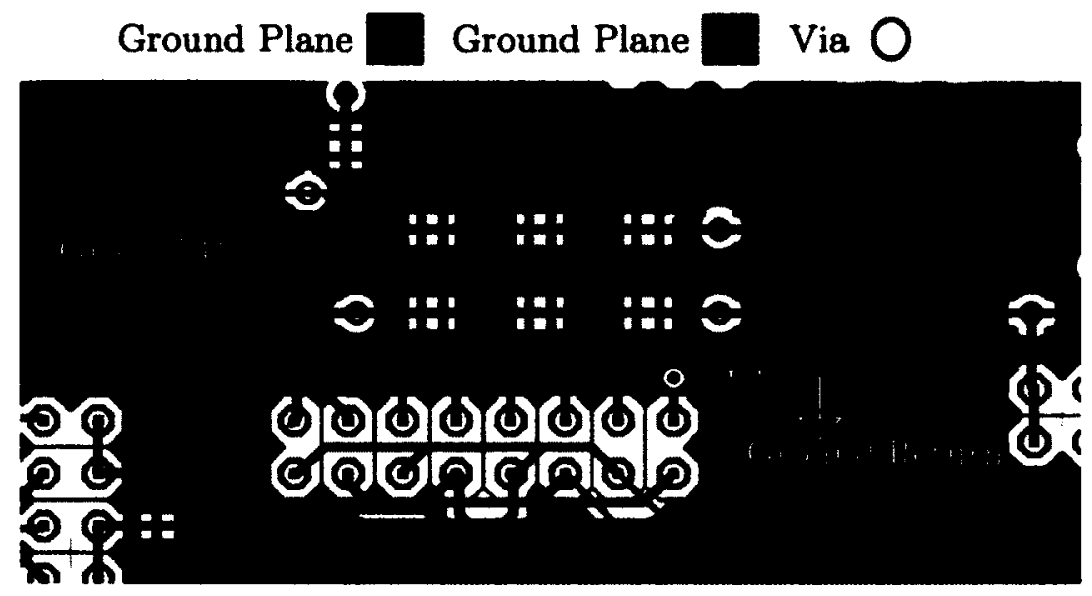

Figure 6.6: Selected area of the PCB showing griding of ground planes to reduce ground loops and inductance in the signal return path.

center of the PCB. "JP11" is an IDE header which is used to connect the output of the ADC to the external digital sampling oscilloscope. The shift register inputs "WE", "CAL", "D" are fed through a SCSI connector at the top of the board. The differential inputs to the ADC as well as the global CLK signals are connected through the gold plated SMA connectors. The traces are well below the maximum trace length as discussed in Sec. 6.2.2.

Both sides of the board are covered with a ground plane as well as scattered vias connections between the ground planes to reduce return path inductance in a process called "griding" [50] as discussed in Sec. 6.2.2. Four layer boards offer the potential to include power planes and ground planes within the internal layers of the PCB. Ideally signals must be routed directly above a ground plane. Given the significantly longer and expensive fabrication process only two layer boards were designed for the ADC. In spite of that, by paying extra care in signal routing and grounding, a similar performance can be achieved even with a two layer board.

The backside of the PCB is presented in Fig. 6.8. The only components on the back side of the PCB belong to the power supply decoupling network. Having multiple 


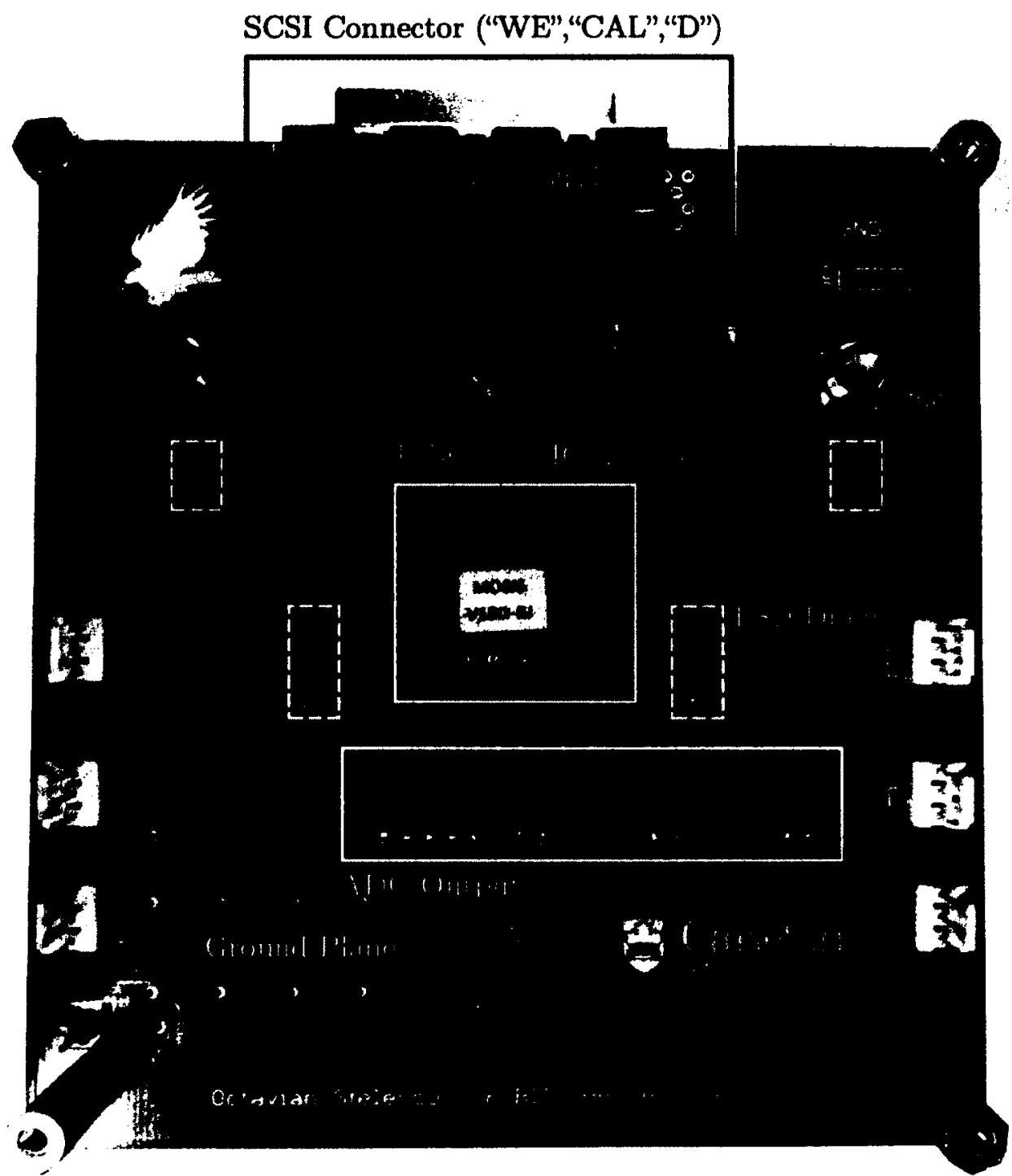

Figure 6.7: Photograph of the topside of PCB1. The SCSI connector is used for the shift register inputs "D", "CAL", "WE". The ADC CLK and the differential inputs used gold plated SMA connectors. The ADC is in-package and soldered at center of the PCB. The output of the ADC uses a 40 pin IDE connector.

power supply and ground pins on the IC reduces the effects of ground bounce from large signal digital switching being coupled into the ground. The same power supply network is used for each power pad; a ferrite bead in series and three capacitors of varying device size and material as discussed in Sec. 6.2.1.

A layout image for both the top side and the bottom side of the PCB is shown 
in Fig. 6.9. The signal routing and the "griding" between the ground planes is more evident in Fig. 6.8. The bill of materials for the PCB is presented in Tb. A.1.

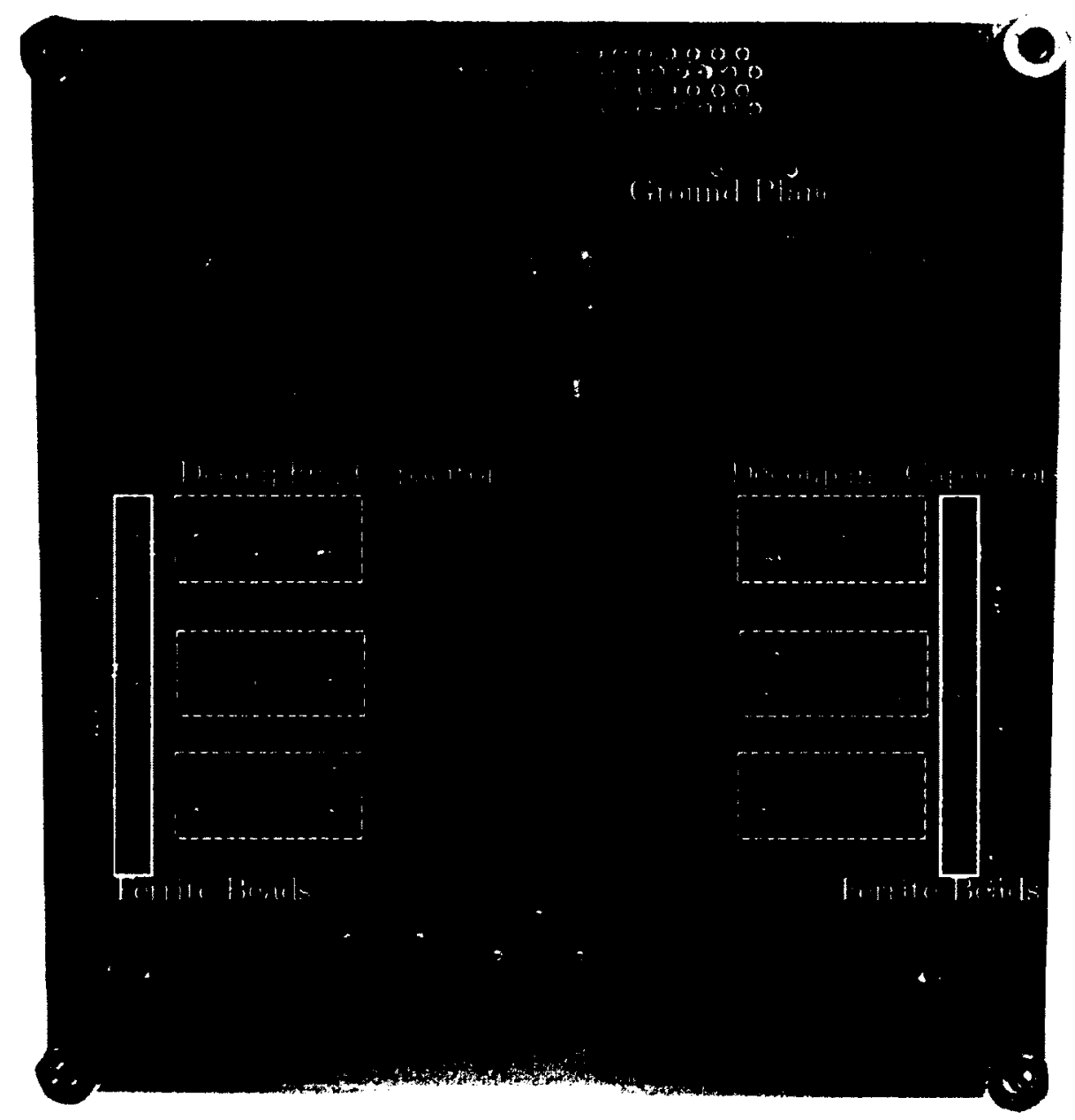

Figure 6.8: Photograph of the back side of PCB1. The decoupling capacitors and the ferrite beads are placed on the back side of the PCB. 

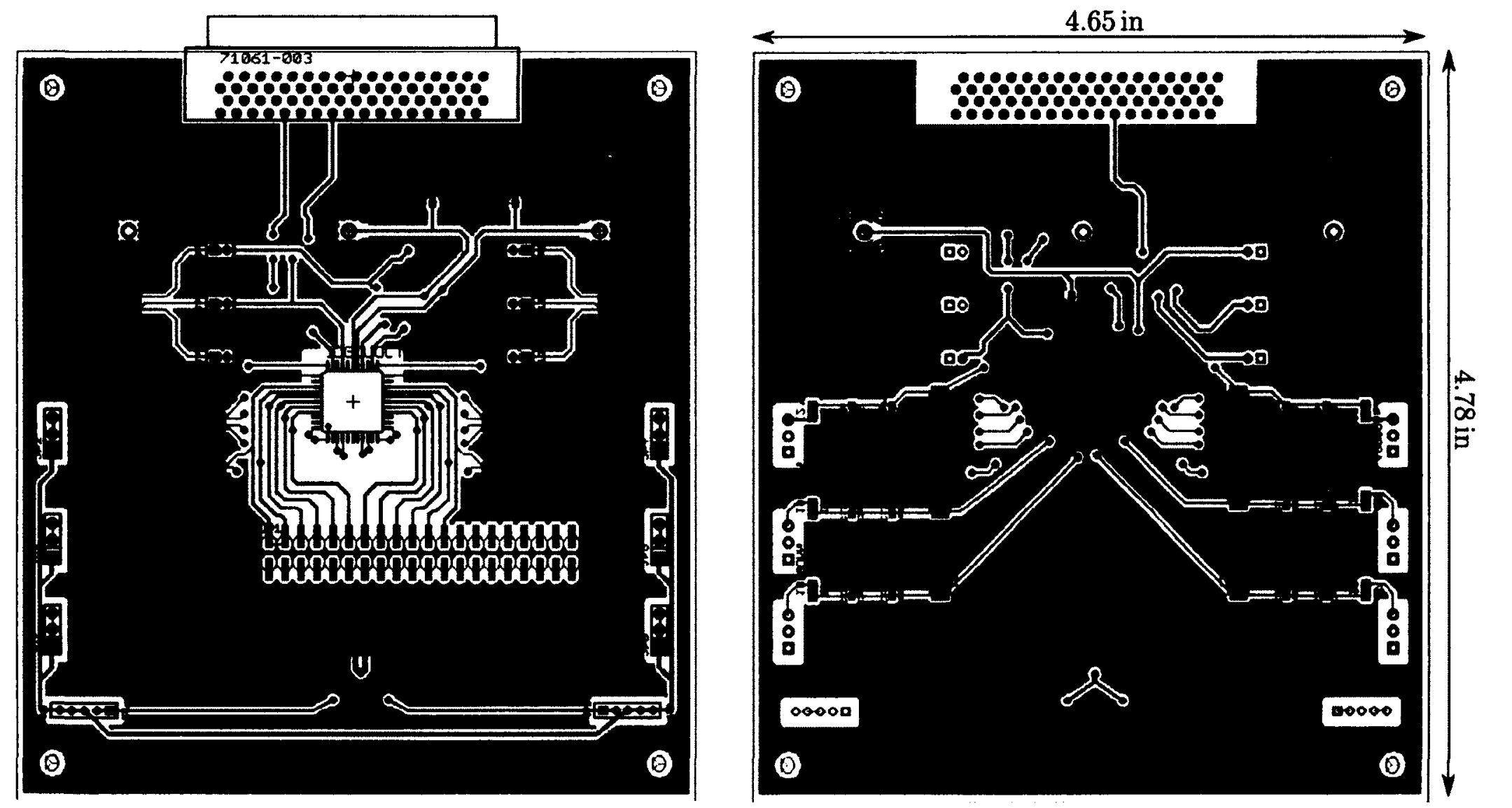

园

Figure 6.9: PCB layout, topside pictured left, bottom side pictured right. 


\subsubsection{Secondary PCB Design with External SAH, "PCB2"}

Without an external SAH, the ADC input is prone to change as the asynchronous decision propagates through the comparison stages causing erroneous codes to be sampled, making the use of an external SAH absolutely necessary. With this in mind the second PCB was designed for maximum flexibility. The PCB features two separate monolithic IC SAH circuits on the PCB with three signal paths designed to bring the output of the SAH ICs into the range of the ADC. As well the PCB has the capability of running from a battery powered supply or directly from source meter power supplies. The SCSI connector was dropped in favor of a space saving IDE connection and an optional SPI compatible interface.

\subsubsection{Signal Paths for Generation of Sampled Differential Inputs}

The first path, "Signal Path 0" is presented in Fig. 6.10. The SAH for this signal path has a short acquisition time of $250 \mathrm{~ns}$ but a long settling time of $2 \mu \mathrm{s}$ [51]. The total combination of acquisition and settling time, or the total 'output time' after the falling edge of SAH clock "S/H", touT, has to be less than half of the clock cycle of the ADC. Therefore, the maximum frequency of operation of the ADC achievable using this SAH at its full scale input is approximately $250 \mathrm{kHz}$. The input and the output of the DS1843 is between $0 \mathrm{~V} . .1 \mathrm{~V}$ and operates on a power supply of $5 \mathrm{~V}$. The output of the DS1843 is then connected to an "ADC driver" developed by Analog Devices [52].

The ADC driver, "AD8139", serves the function of level shifting the output of the SAH to the mid-rail value of the ADC determined by the $\mathrm{V}_{O C M}$ input. To maintain the highest resolution achievable from the $\mathrm{SAH}$, the input is driven at the maximum full scale input. As a consequence the output of the DS1843 must be scaled and level 


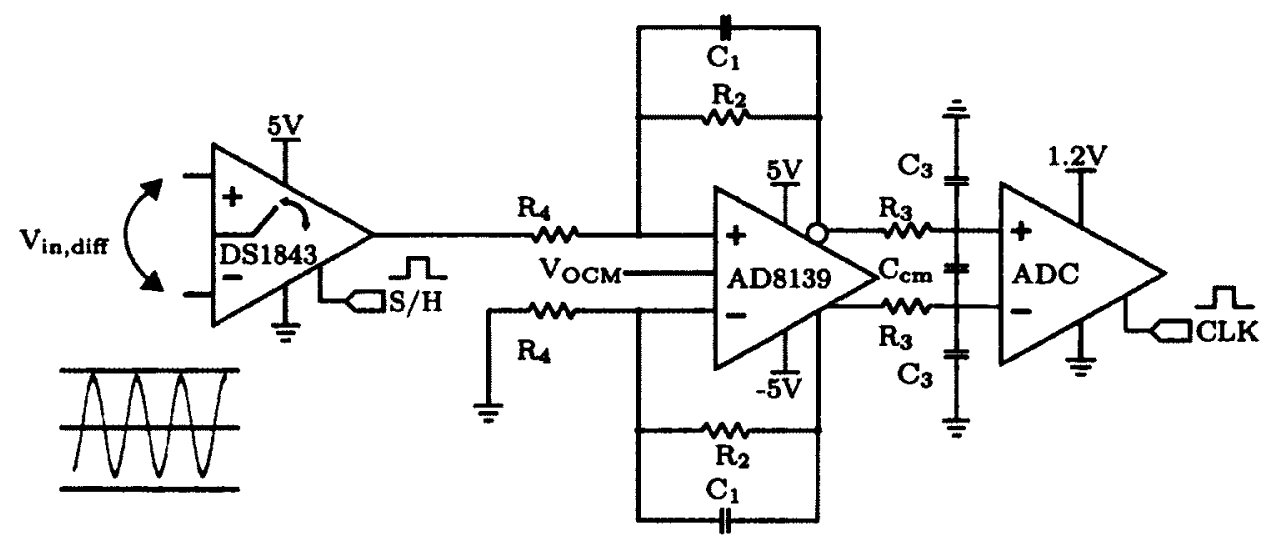

Figure 6.10: PCB2 signal path 0 . The "DS1843" is a SAH with an output between $0 \mathrm{~V} . .1 \mathrm{~V}$. The "AD8139" ADC driver provides; level tuning, single ended to differential conversion, and fixed signal attenuation.

shifted to bring it into the input range of the ADC. The AD8139 implements the level shifting function, centering the output of the $\mathrm{SAH}$ around $\mathrm{V}_{\mathrm{OCM}}$, as well as converting the input from single ended to differential. In addition the ADC driver provides gain attenuation through a resistive feedback network. To filter high frequency noise, at the input of the ADC, a basic one pole low pass filter is implemented using series resistances and shunt capacitors $\left(\mathrm{R}_{3}\right.$ and $\left.\mathrm{C}_{3}\right)$. Common mode filtering is accomplished through the $\mathrm{C}_{\mathrm{cm}}$ capacitor.

The remaining signal paths use a common SAH, the "AD783" [53] produced by Analog Devices. Compared to the first SAH the AD783 has a much higher frequency of operation of $15 \mathrm{MHz}$ [53]. The AD783 operates on a $-/+5 \mathrm{~V}$ power supply with a single ended input and output between $-2.5 \mathrm{~V} . .2 .5 \mathrm{~V}$. Signal path 1 presented in Fig. 6.11 shows the output of the AD783 connected through a high frequency low input/output offset high speed operational amplifier (op-amp), the "ADA4853" [54].

The ADA4853, connected in a non inverting configuration implements an adjustable signal attenuation using a resistive feedback network in the form of a resistive potentiometer, $\mathrm{R}_{\text {trim }}$. The ADA4853 brings the SAH output into the input range of 


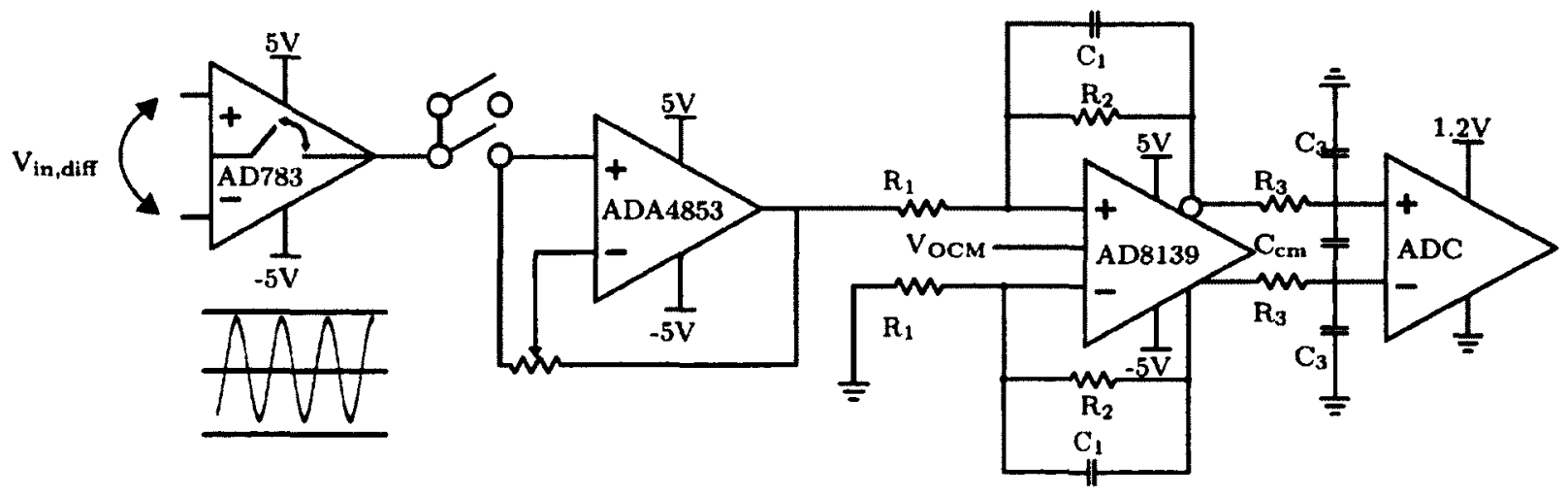

Figure 6.11: PCB2 signal path 1. The "AD783" is SAH with an output between $-2.5 \mathrm{~V} . .2 .5 \mathrm{~V}$. The "ADA4853" provides adjustable gain through $\mathrm{R}_{\text {trim }}$. The AD8139 handles level shifting and conversion to differential output.

the ADC. The output of the ADA4853 is connected to the same type of ADC driver used in Signal Path 0 along with its associated low pass filter output.

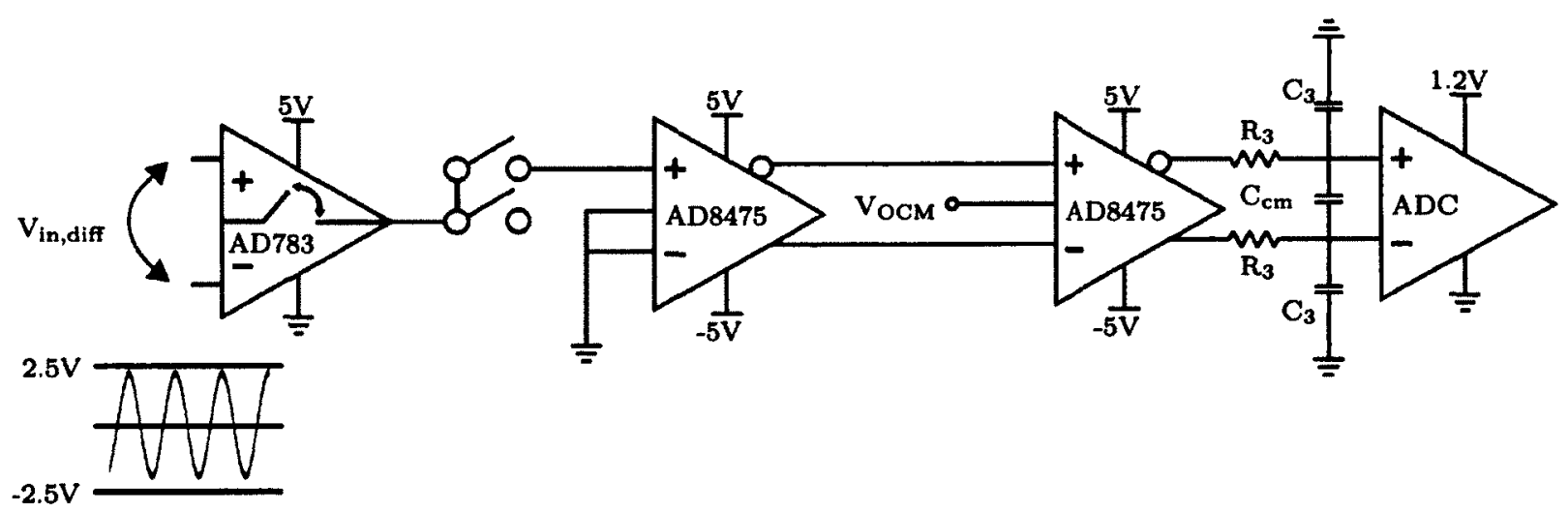

Figure 6.12: PCB2 signal path 2. The "AD783" is SAH with an output between $-2.5 \mathrm{~V} . .2 .5 \mathrm{~V}$. Two cascaded "AD8475s" provide an attenuation of 0.16 as well as single ended to differential conversion.

The final signal path, "Signal Path 2" uses a bullet proof approach for bringing the output of the SAH into the input range of the ADC. The AD8475 [55] is a high quality $\mathrm{ADC}$ driver with a maximum output offset of $500 \mu \mathrm{V}$, less than LSB/4 of the ADC. The AD8475 uses built in laser trimmed resistors to achieve a gain of $0.4 \mathrm{~V} / \mathrm{V}$ with excellent phase matching. The signal path uses two cascaded AD8475s 
implementing a total gain of $0.16 \mathrm{~V} / \mathrm{V}$ out of the required $0.06 \mathrm{~V} / \mathrm{V}$. The rest of the gain attenuation is provided by not driving the $\mathrm{SAH}$ at a full scale input. Only one signal path is necessary at a given time to drive the ADC.

\subsubsection{Clock Generation}

The SAH must acquire the input input and settle before the rising edge of the ADC clock. As well, the duty cycle of the SAH must encompass the output time and duty cycle of the ADC clock $^{70}$. This means that the ADC requires a separate clock from the ADC clock. The second PCB is designed to allow for the $\mathrm{S} / \mathrm{H}$ clock signal to be driven by an additional function generator which has its $10 \mathrm{MHz}$ reference tied to the ADC clock signal generator.

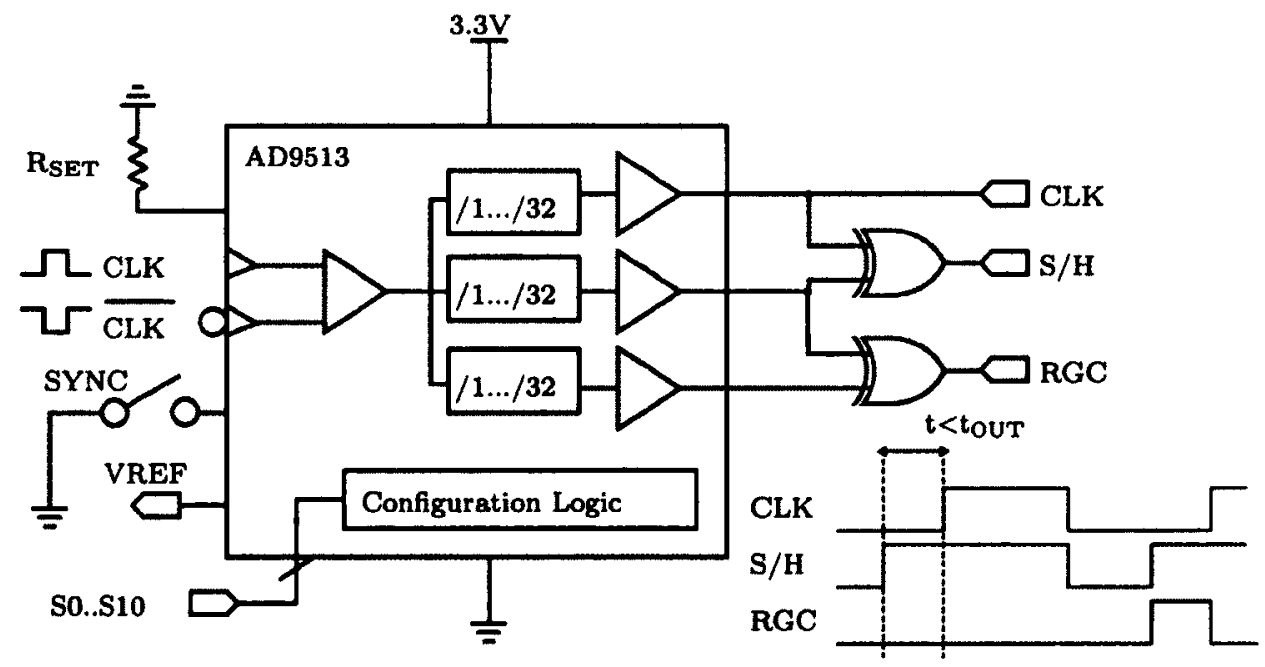

Figure 6.13: Functional diagram of PCB2 timing using the "AD9513" and XOR gates. The "AD9513" is frequency divider with adjustable phase, duty, cycle for three output channels. "CLK" is the ADC clock signal, "S/H" is the SAH clock, "RGC" is the 8-bit register clock.

The output of the ADC can be taken from level shifting digital translator, the "ADG3308" [56] or after an optional, 8-bit clocked register, the "CY74FCT2574T" [57].

\footnotetext{
${ }^{70}$ This discussion is in reference to the AD783 SAH.
} 
The 8-bit register requires an additional clock signal "RGC". To decrease the overhead of PCB timing generation using external function generators, the AD9513 [58] can be used in combination with 2 XOR gates to generate the timing signals. The AD9513 is an IC clock divider with delay and phase adjustment that are programable through 10 bits of four level logic.

\subsubsection{Layout of PCB2}

The layout of the top side of PCB2 is presented in Fig. 6.14. Similar to the first PCB, a ground plane is created on both sides of the PCB and joined through vias to reduce ground loops. Orphaned areas without a direct path to ground are connected through vias to the ground plane on the other side of the PCB.

At the center of the PCB above the ADC, labeled "ICGCUOCT" on the PCB, there is signal path select jumper which is used to chose between the three different signal paths. Signal paths 1 and 2 which share the same SAH are at the top of the board along with "SING" jumper which selects the active path 1 or 2 . The bottom of the ADC is used to hold the "AD9513" which is programmed by 10 bit jumpers using four level logic ${ }^{71}$. Four separate voltage regulators are provided on the board to generate the required supply voltages if the board runs from battery power. Power supply traces were widened to decrease series resistance and inductance and provide free capacitive decoupling. The back side of the PCB is also presented in Fig. 6.14. The back side of the PCB serves mainly as a ground plane with only a handful of components being placed on the back side. A photograph of PCB2 populated with components is shown in Fig. 6.15.

\footnotetext{
${ }^{71}$ The logic levels are $0,1 / 3 V_{S}, 2 / 3 V_{S}$, and $V_{S}$.
} 

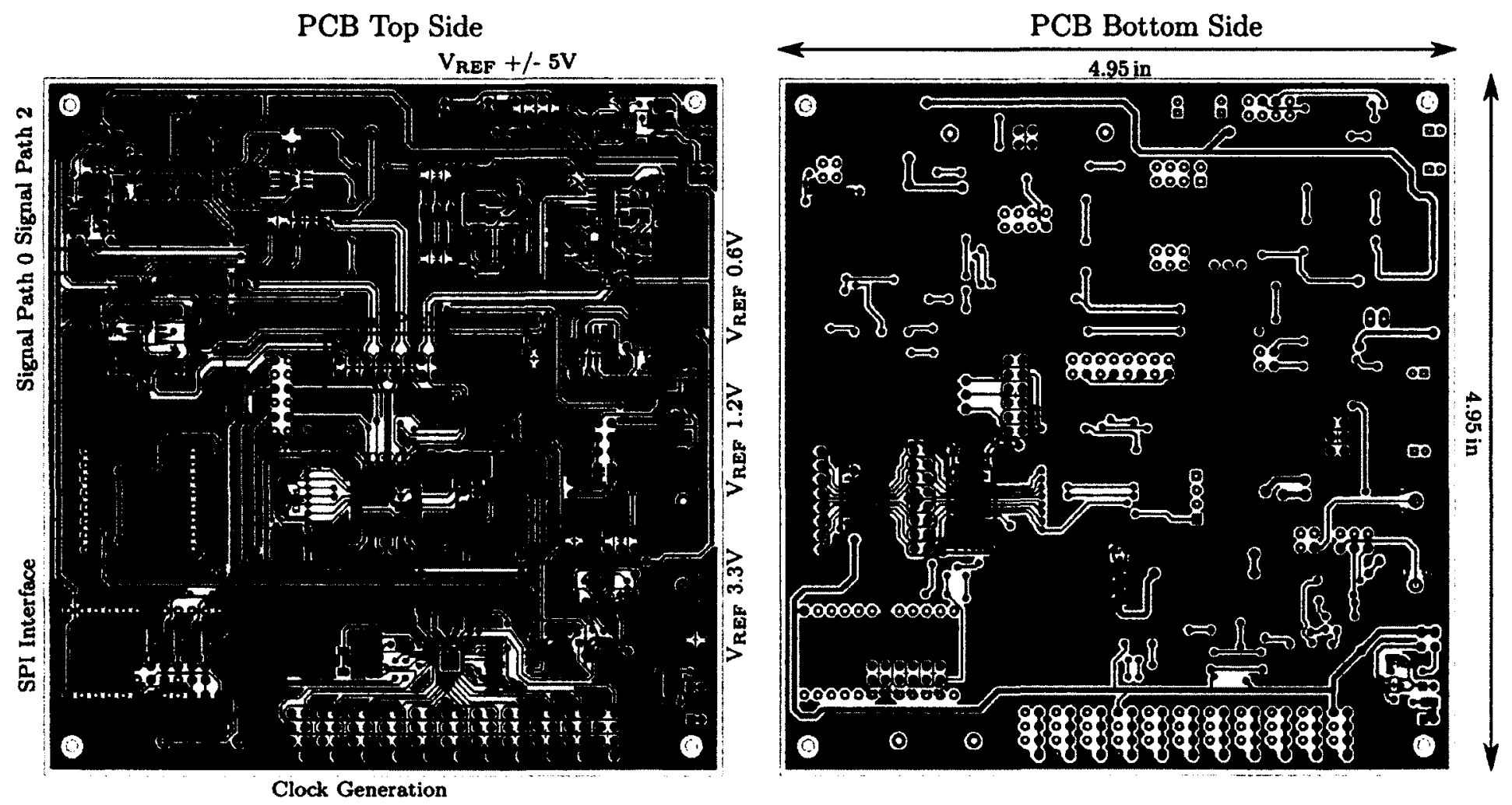



Figure 6.14: Layout of PCB2, top side pictured on the left, bottom side pictured on the right. 


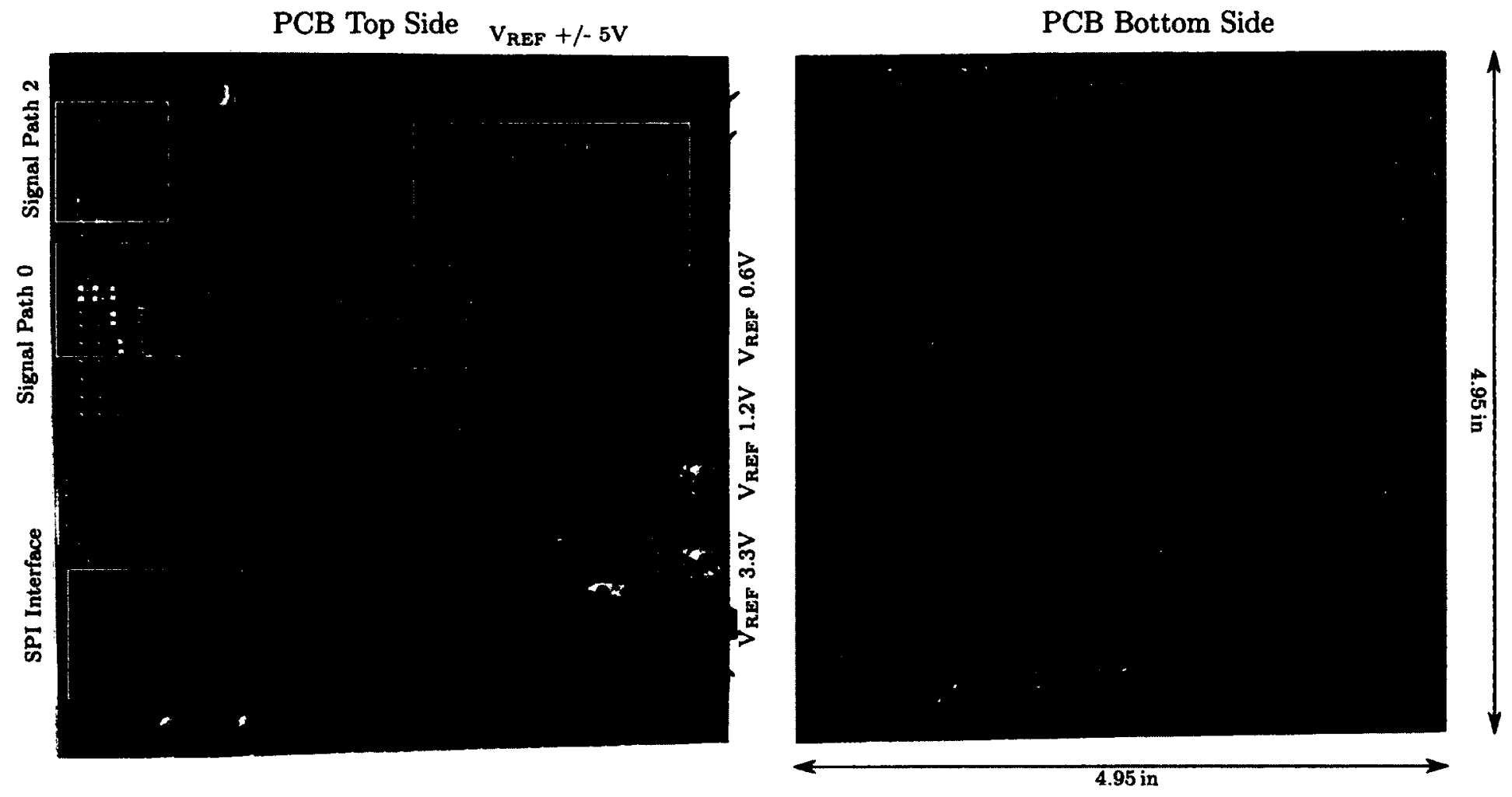

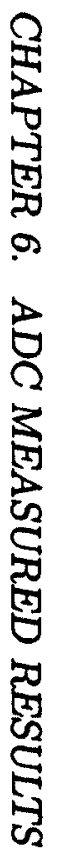

Figure 6.15: Photograph of PCB2, top side pictured on the left, bottom side pictured on the right. 


\subsection{Preliminary Testing}

Preliminary testing of the ADC was carried out using PCB1. After the board was assembled and all connections where checked for shorts, the output of the ADC was observed using the test configuration depicted in Fig. 6.3. The output of the ADC showing all the bits switching and active is presented in Fig. 6.16.

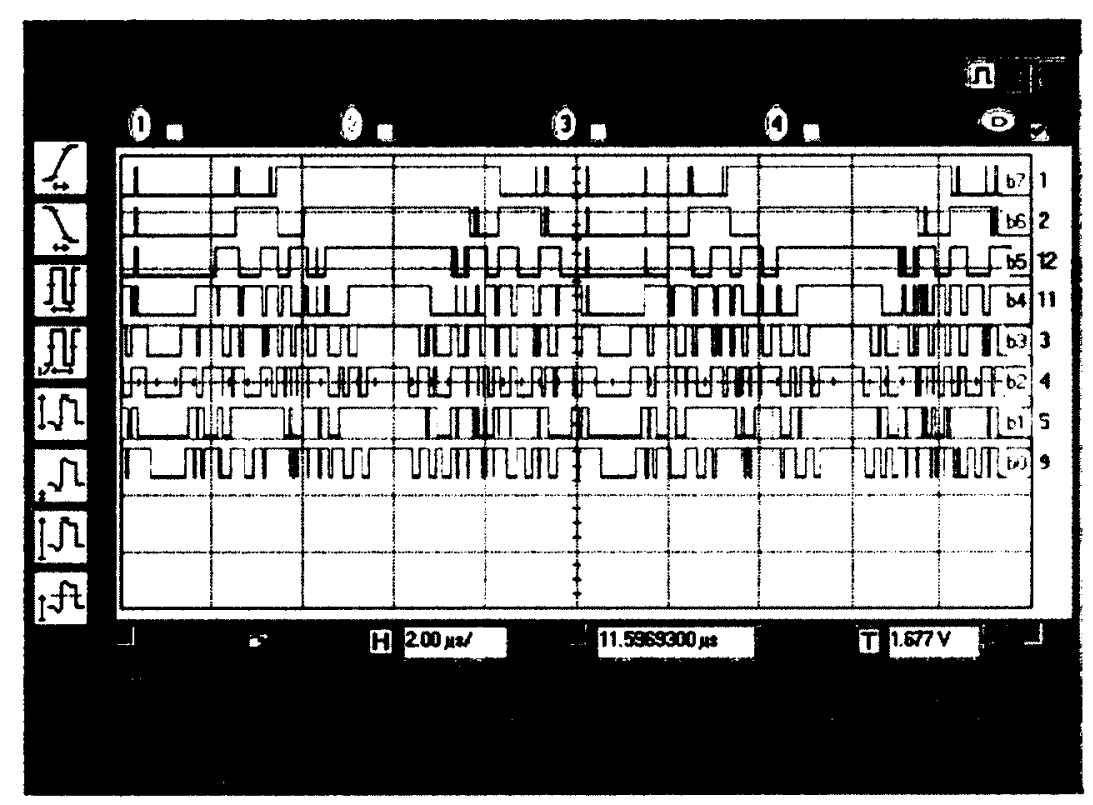

Figure 6.16: Screenshot from the DSO showing the ADC output bits for an over driven sinusoidal input of $100 \mathrm{kHz}$ and sampling frequency of $20 \mathrm{MHz}$.

When the ADC output was reconstructed to be evaluated for INL and DNL measurements, the presence of large spikes appeared on the reconstructed output as well as non monotonic code transitions. An example of this can be seen in Fig. 6.17. Non monotonic code transitions are expected if the ADC does not have the optimum calibration code. The spikes which appear on the reconstructed output have the highest amplitude near the code transitions. The code transitions correspond to an MSB switch from a logic "0" to a logic " 1 " or conversely. This corresponds to transition of the digital word from "0111111" to " 1000000 " or the reverse. Since the differential 
input to the comparators is only held by the capacitance of the differential trace, this capacitance is subject to change as the decision propagates through the stages of the ADC.

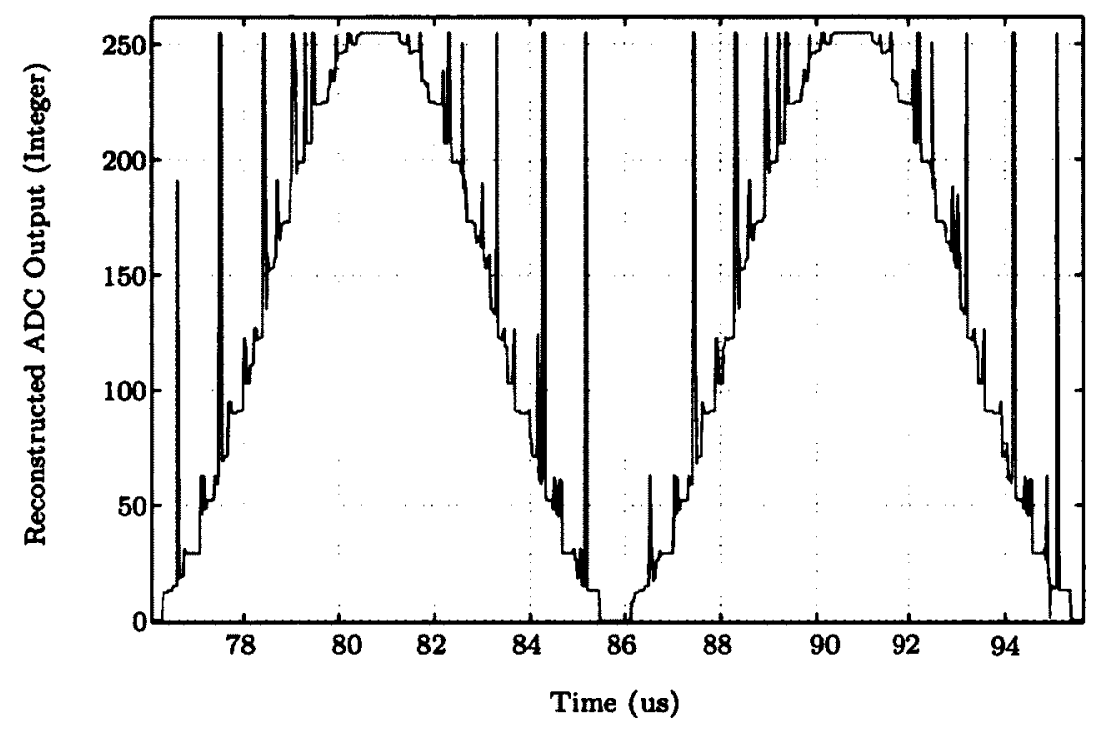

Figure 6.17: Reconstructed output for fabricated ADC showing code transition sampling.

This behavior was verified through simulation of the ADC and is presented in Fig. 6.18. In this simulation the ideal SAH circuit was removed and the simulation was performed with "conservative" simulation settings shrinking the size of the simulation time step. Another factor which must be taken into account is that no post layout parasitic extraction could be carried out on the full ADC. Therefore, the input to all the comparators changes instantaneously making this effect less pronounced in simulation. The appearance of spikes at code transitions is not a failure of the ADC to function as required. The appearance of code transition spikes can be removed through the implementation of a SAH at the front end of the ADC. For the reasons discussed in this chapter the second SAH was designed and presented in Sec. 6.2.4.

Not only is the dynamic analysis of the ADC impacted by the lack of a SAH but 


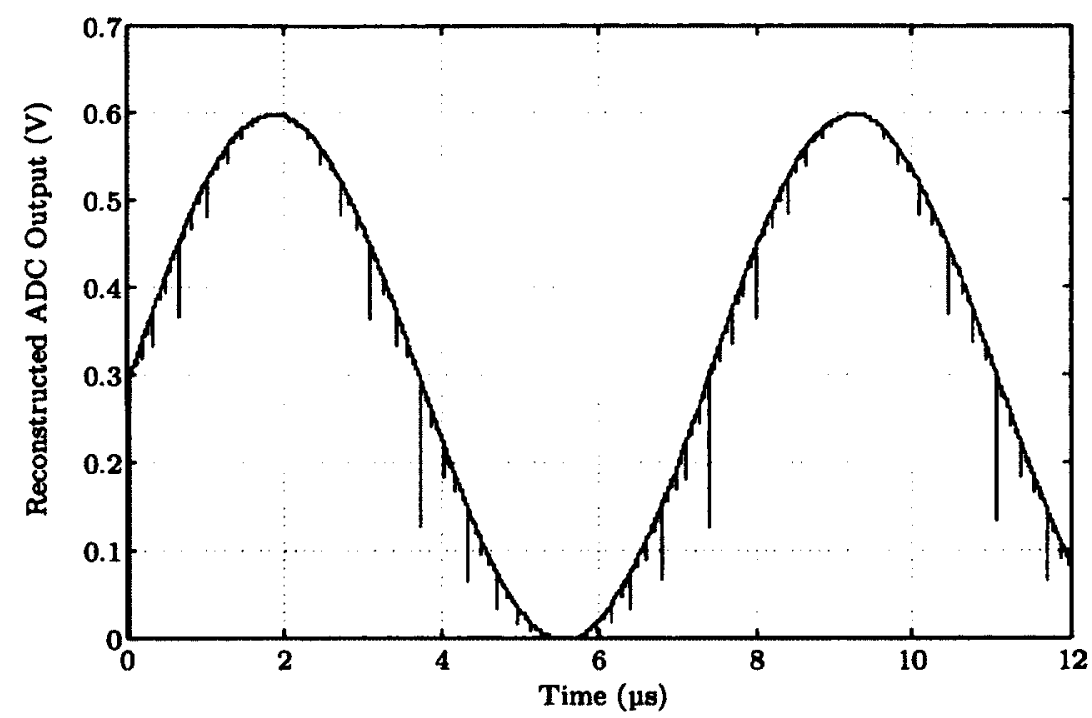

Figure 6.18: Reconstructed output for simulated ADC showing code transition sampling. The SAH was removed and the simulation was performed with conservative simulation settings.

DC testing as well is affected by sampling code transitions or rather, having the input change during a conversion. The measured noise from the equipment was on the order of a few LSBs, more than enough to cause pronounced code transitions even for DC static analysis.

Notice that the spikes in the simulation plot are in the opposite direction compared to the measured behavior in Fig. 6.17. This is not of fundamental importance but can be partly attributed to the way in which the ideal VHDL DAC makes its conversion. As well the simulations were performed without parasitic extraction. If a full IC simulation with extracted parasitics was performed, it is conceivable that the spikes would appear in the opposite direction as the bits arrive at slightly different times at the input to the ideal DAC. What is key of note is that code transition spikes appear and can be resolved through the use of a SAH.

The next stage involved in the preliminary testing of the ADC involved programming the shift register of the ADC. This was achieved using the same test bench. 
The CLK and the INP and INN signals were disabled while the shift register was programmed. The result of loading the nominal calibration code can be observed in Fig. 6.19. After the nominal code used to establish the desired comparator thresholds in simulation was loaded into the ADC, the code depth of the output bits grew ${ }^{72}$. This test confirmed the operational functionality of CABS ADC shift register based memory.

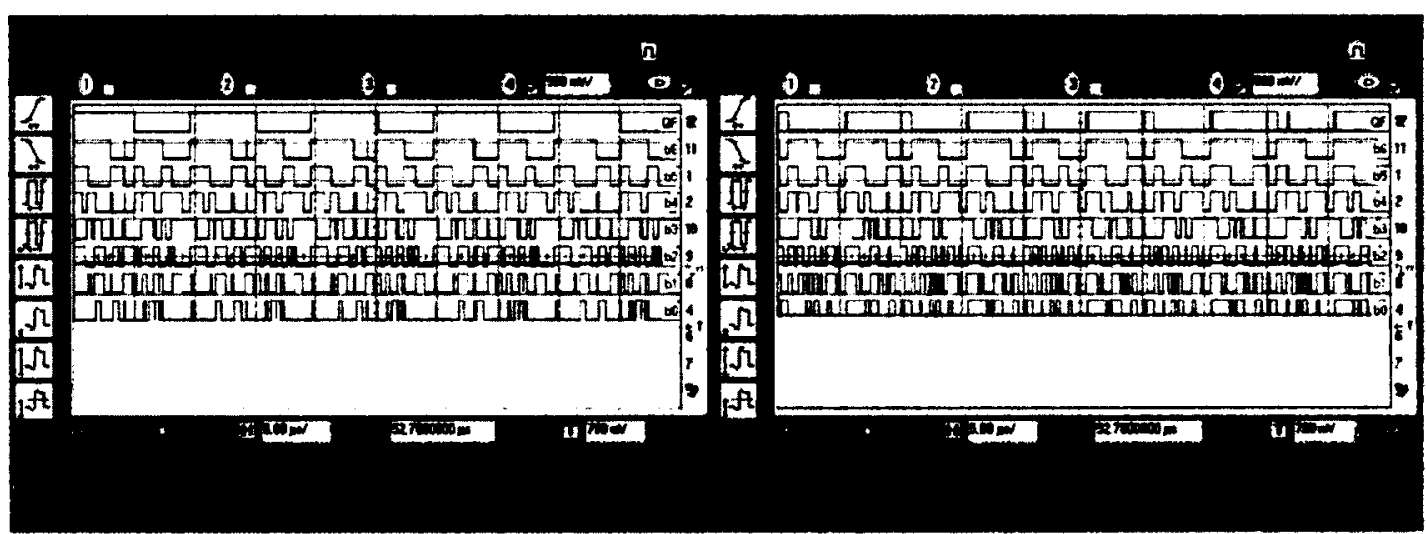

Figure 6.19: Shift register programming of the fabricated ADC. After loading the nominal calibration code, the code depth of the outputs bits is evidently increased.

\footnotetext{
${ }^{72}$ That is to say, more synchronized code transitions became visible indicating an increase in the number of codes, or the code depth
} 


\subsection{Calibration}

The calibration routine described in this section is identical to the calibration routine used to extract the nominal tuning code for the simulated comparator. The Matlab pseudo-code presented in Listing 6.1 has been modified from [26] as the calibration code used in the tuning of this $A D C$ is too vast to give a quick insight into the calibration methodology.

For a desired comparator which requires tuning, nearby comparators in the same stage have their threshold shifted far from the desired value. For the ADC described in this work, the neighboring comparators could have their thresholds shifted by as much as 30 LSB. This ensures that the result of any triggering on neighboring comparators is pushed far from the desired threshold and does not bias the mean of the measured threshold. The measured threshold is sampled using the digital spectrum oscilloscope discussed in the Sec. 6.1 and reconstructed to an analog value using an ideal DAC implemented in software. The stages are progressively tuned, from the fifth stage to the eight stage of the ADC. Each calibration code is then stored in database of comparator structures. The ADC can require a different tuning code depending on the frequency of operation, temperature, maximum input frequency excursion and PVT. 
Listing 6.1: Matlab pseudocode binary search calibration routine for a single comparator.

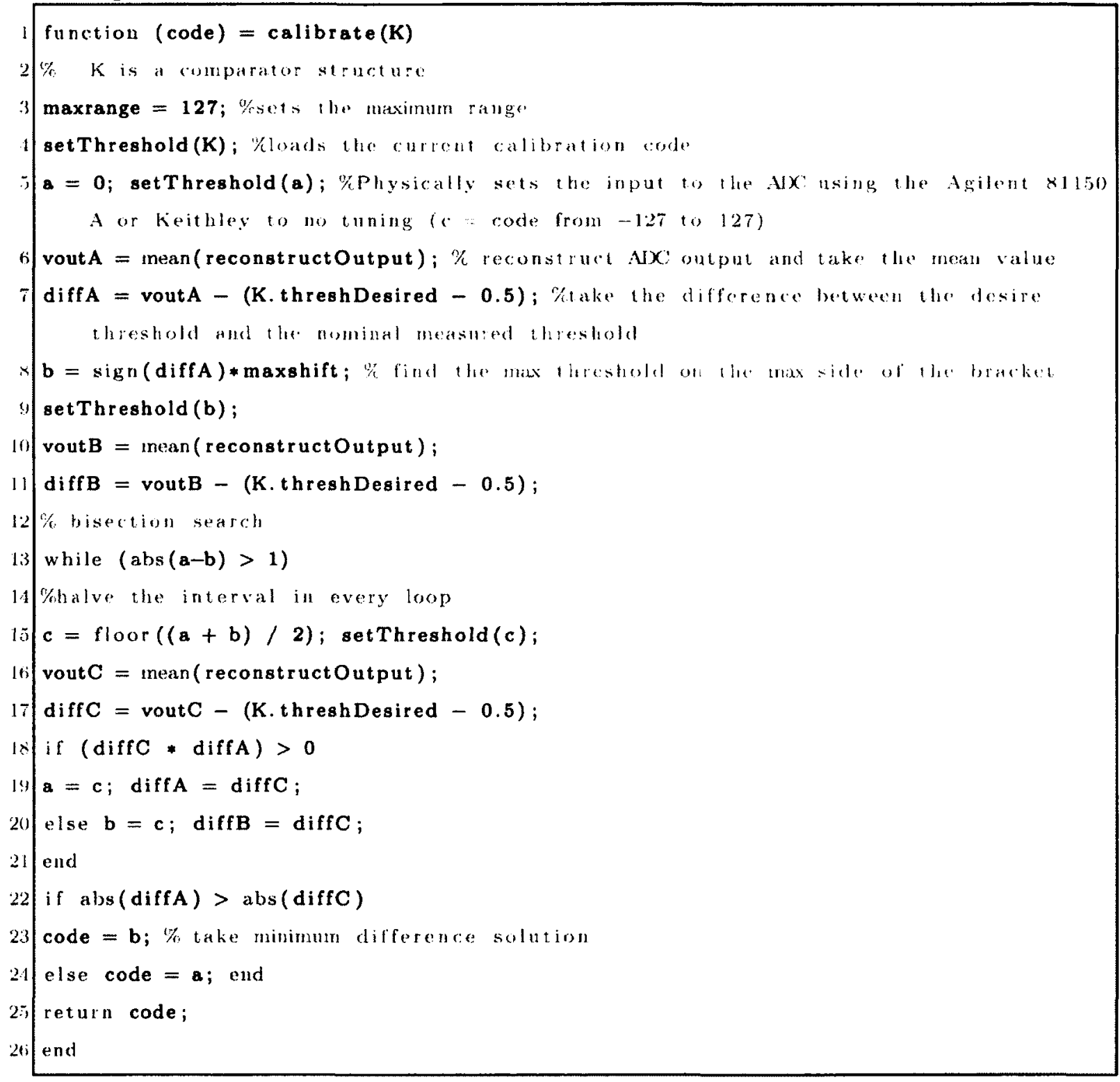




\subsection{Static Linearity Analysis}

\subsubsection{Integral Nonlinearity (INL)}

The INL for the fabricated IC was measured using the technique presented in Sec. 5.1.1 using the second $\mathrm{PCB}$, "PCB2". Two differential ramps corresponding to a duration of $2.5 \mathrm{~ms}$ and a full scale input were used to measured the INL. The result of the INL before calibration is presented in Fig. 6.20 with a worst case INL of 64 LSB.

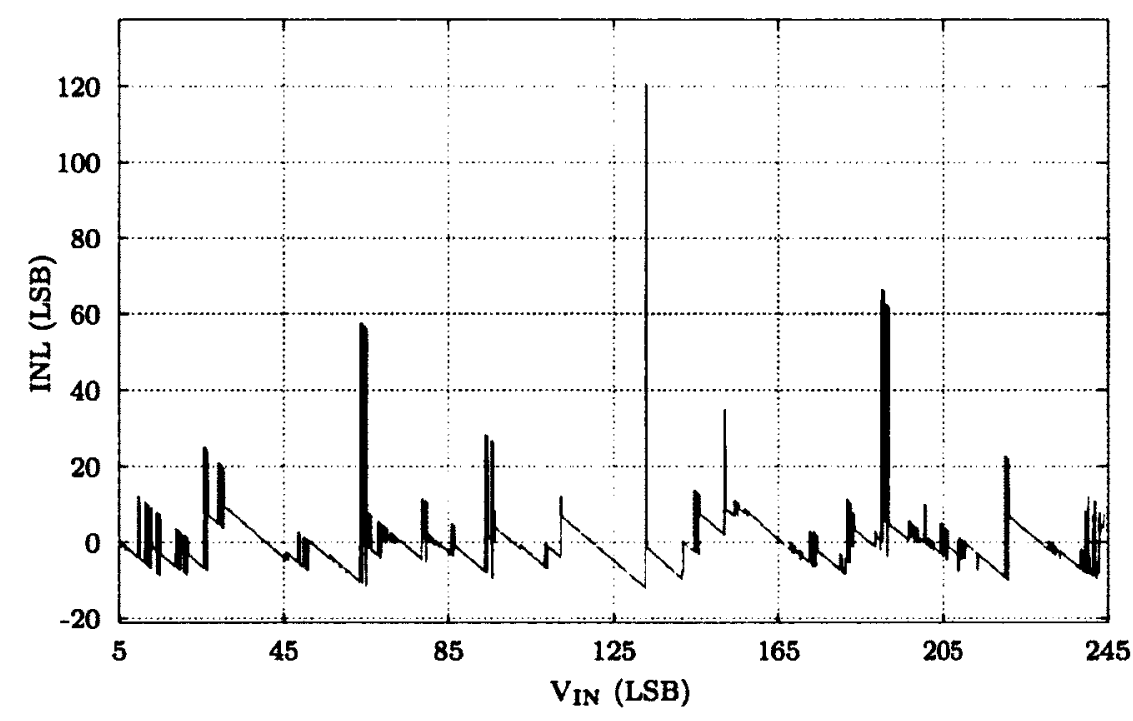

Figure 6.20: Pre-calibration Integral nonlinearity (INL) as a function of input voltage $V_{I N}$, worst case INL is $64 \mathrm{LSB} ; f_{S}=4 \mathrm{MHz}, 2.5 \mathrm{~ms}$ differential ramp.

Using a non-optimum calibration code based on the optimum simulation calibration code, the INL was improved from 64 LSB to 11 LSB. With further optimization based on the calibrations strategy proposed in [26] or using a novel calibration code it is conceivable that the static performance of the fabricated ADC could be further improved. 


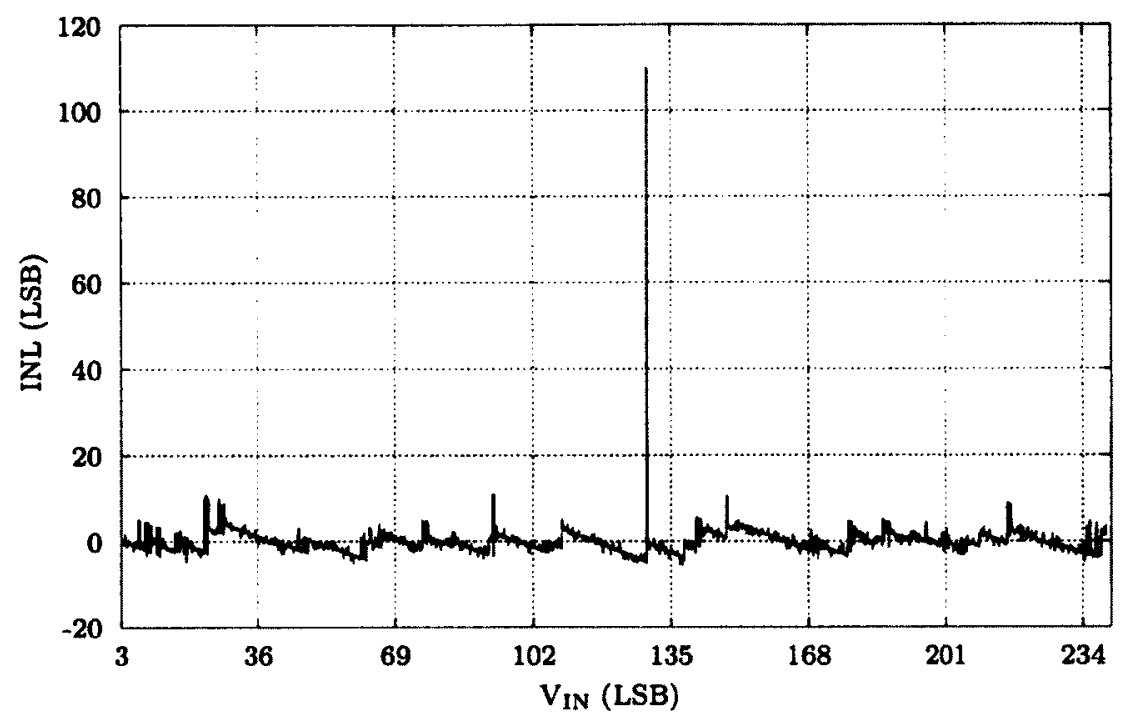

Figure 6.21: Post-calibration Integral nonlinearity (INL) as a function of input voltage $V_{I N}$, worst case INL is $11 \mathrm{LSB} ; \mathrm{f}_{\mathrm{S}}=4 \mathrm{MHz}, 2.5 \mathrm{~ms}$ differential ramp. 


\subsubsection{Differential Nonlinearity (DNL)}

The DNL was extracted for the fabrication IC using the technique outlined in Sec. 5.1.2. Similar to the INL the performance of the DNL was improved by using an improved calibration code albeit a non-optimum calibration code from -68 LSB in Fig. 6.22 to 14 LSB in Fig. 6.23.

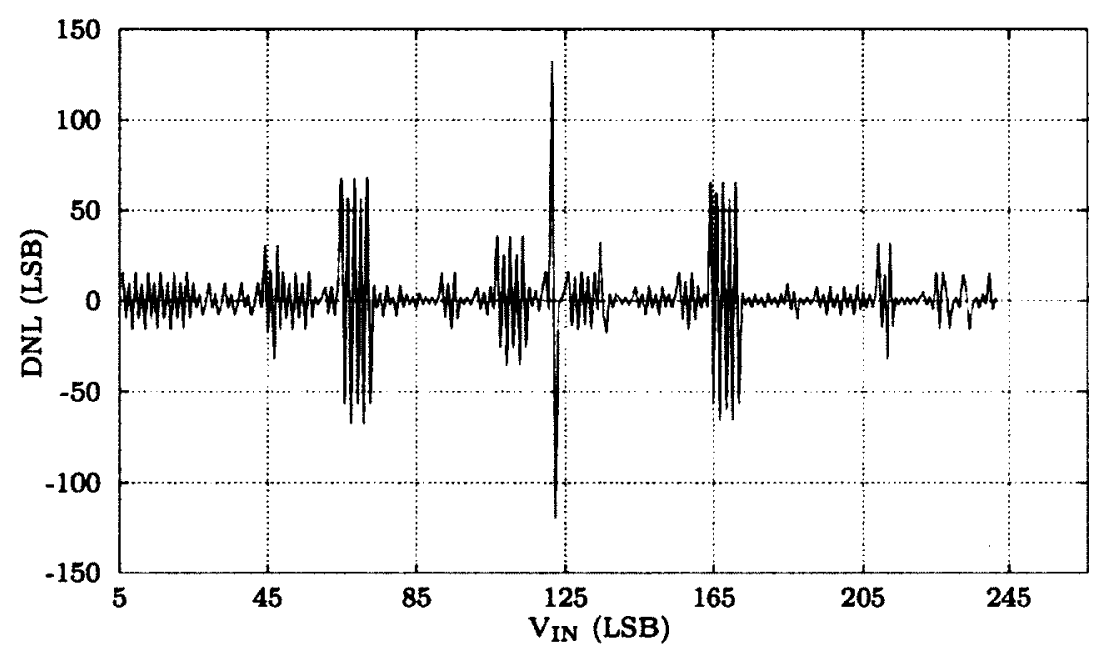

Figure 6.22: Pre-calibration differential nonlinearity (DNL); worst case DNL is $-68 \mathrm{LSB} ; \mathrm{f}_{\mathrm{S}}=4 \mathrm{MHz}, 2.5 \mathrm{~ms}$ differential ramp.

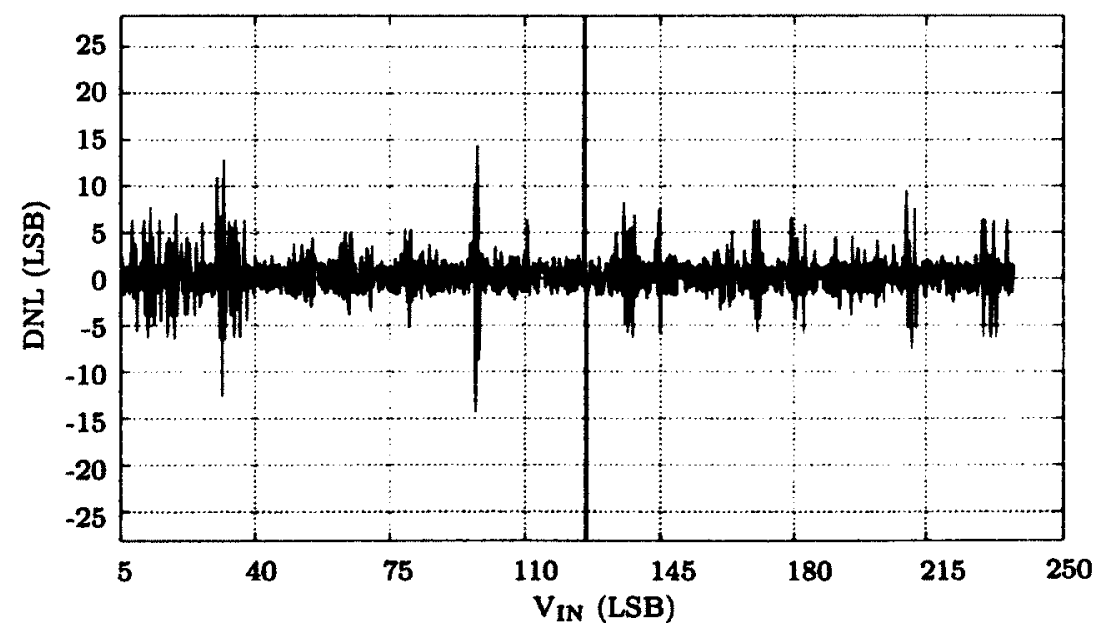

Figure 6.23: Post-calibration differential nonlinearity (DNL); worst case DNL is $14 \mathrm{LSB} ; \mathrm{f}_{\mathrm{S}}=4 \mathrm{MHz}, 2.5 \mathrm{~ms}$ differential ramp. 


\subsection{Measured Dynamic Analysis}

The dynamic performance metrics of the fabricated CABS ADC were measured and analyzed using the same approach presented in Sec. 5.2.2. Dynamic analysis measurements were performed using a sampling frequency of $\mathrm{f}_{8}$ of $4 \mathrm{MHz}$ and an input frequency $f_{i}$ of $50 \mathrm{kHz}$. An FFT spectrum for the recontracted output of the ADC is presented in Fig. 6.24. The ENOB using an improved but non-optimum calibration code was improved to 5.1 bits. Without any calibration the ENOB was 3.1 bits. The SINAD was $32.3 \mathrm{~dB}$ with a SFDR of $35.7 \mathrm{~dB}$. The maximum sampling frequency was limited by the operation of the SAH which requires a maximum settling time of $250 \mathrm{~ns}$ for a full scale input. With an internal, on-chip SAH amplifier or a faster SAH it would be possible to test the fabricated ADC at a higher sampling frequency as was verified by preliminary testing using PCB1 with no SAH.

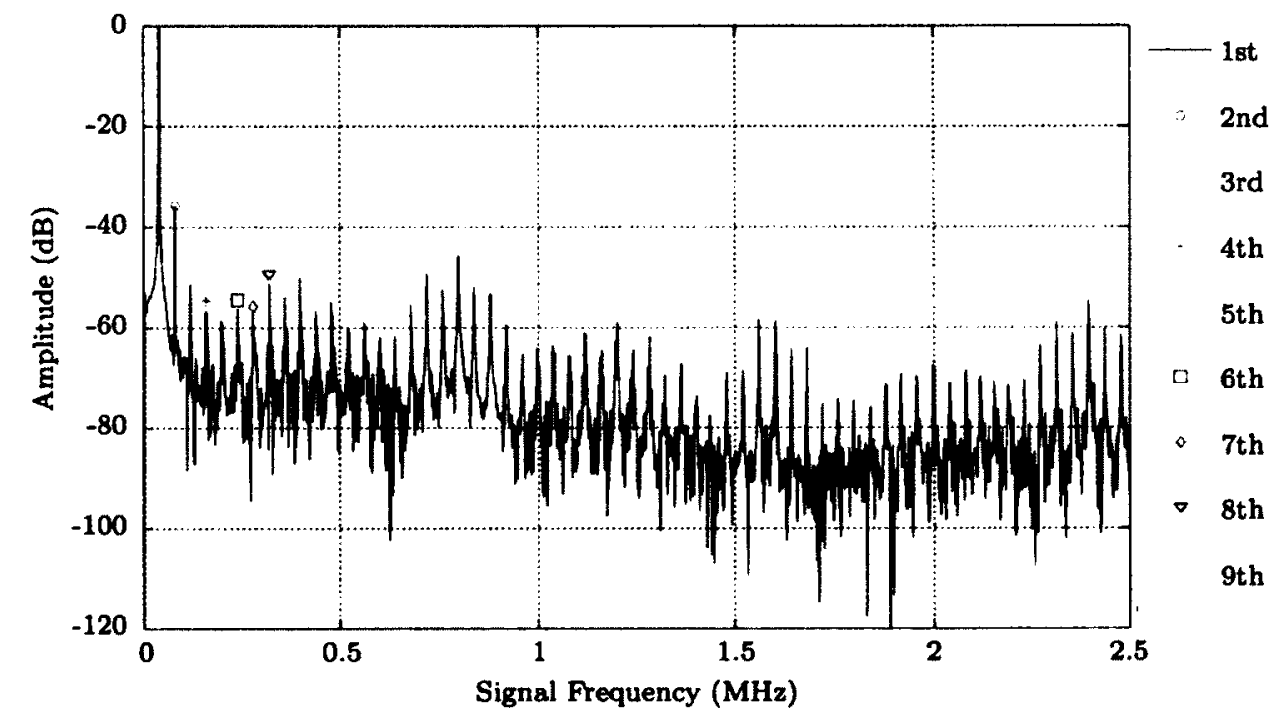

Figure 6.24: Post calibration FFT spectrum of the input signal, $f_{s}=4 \mathrm{MHz}$, $\mathrm{f}_{\mathrm{i}}=50 \mathrm{kHz}, \mathrm{n}=7, \mathrm{~m}=1024, \mathrm{ENOB}=5.1, \mathrm{SINAD}=32.3 \mathrm{~dB}, \mathrm{SFDR}=35.7 \mathrm{~dB}$ 


\subsection{Power Dissipation}

The total measured power consumption versus sampling frequency for the IC including output buffers for a fixed input frequency, $\mathrm{f}_{\mathrm{i}}$, of $98.3 \mathrm{KHz}$ is presented in Fig. 6.25. The power consumption follows a steady, nearly linear increase up until $35 \mathrm{MHz}$. Slightly before this point the ADC reaches its maximum frequency of operation, and the output clock does not trigger the comparator responsible for $\mathrm{b} 7$ in the eight stage. As the frequency is increased, the latter stages of the ADC are not triggered, reducing the switching activity of the output latch and buffer, and reducing the power consumption.

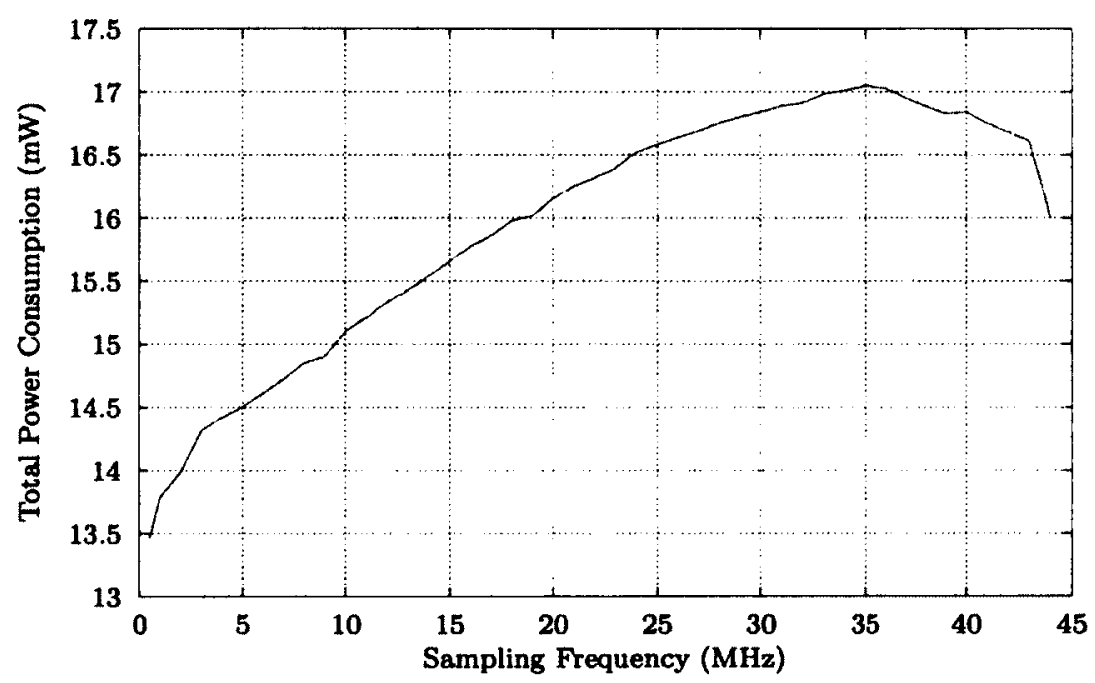

Figure 6.25: Total measured power consumption, as a function of the sampling frequency $f_{s}$ for an input frequency of $f_{i}=98.3 \mathrm{KHz}$

The total measured power consumption for the full IC for Nyquist operation is presented in Fig. 6.26. The input frequency for the Nqyuist frequency is selected to be a non integer multiple of the sampling frequency to avoid undesired aliasing effects. The total Nyquist power consumption is higher than the total power consumption presented in Fig. 6.25 as the switching activity of the output buffers and core is 


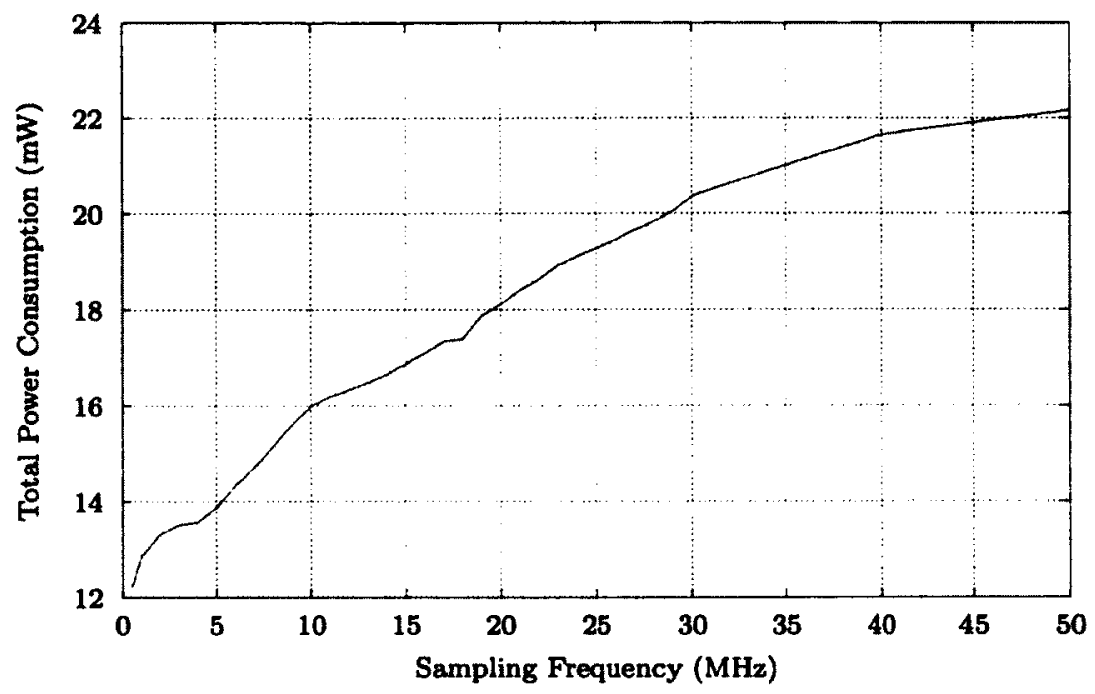

Figure 6.26: Total measured power consumption, as a function of the sampling frequency $f_{s}$ at Nyquist input frequency.

increased.

Finally, the measured FOM for the power consumption of the ADC core as function of a fixed input frequency of $98.3 \mathrm{KHz}$ is presented in Fig. 6.28. At the desired frequency of operation of $25 \mathrm{MHz}$ the total energy per conversion is $15 \mathrm{fJ} /$ conversion.

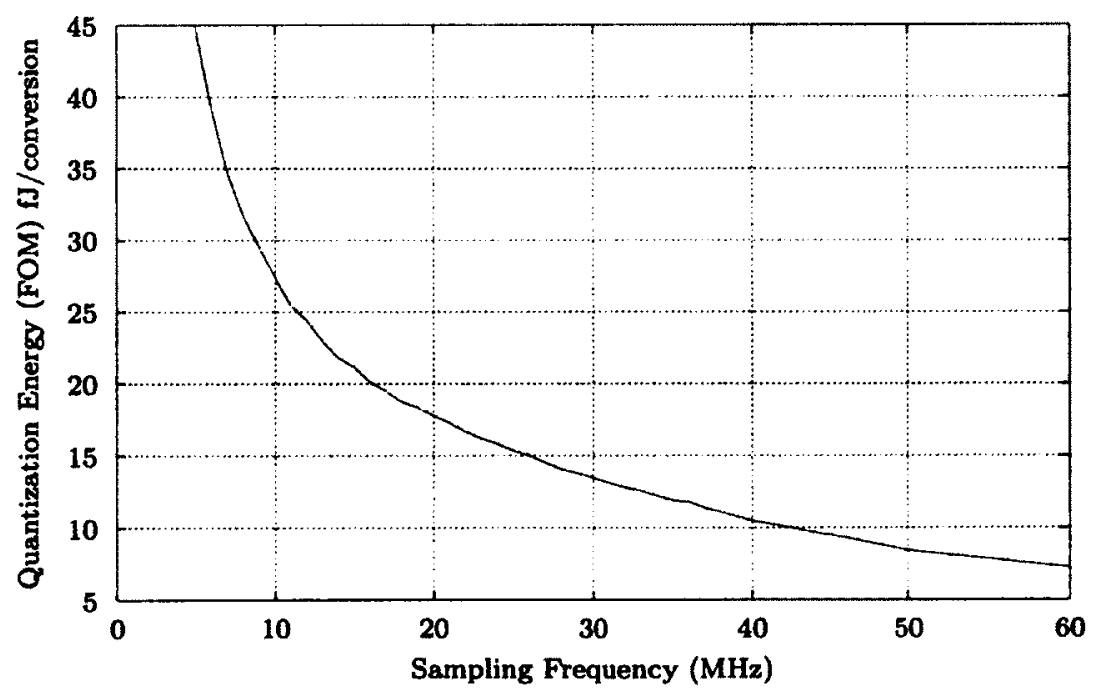

Figure 6.27: FOM, as a function of the sampling frequency $f_{s}$ for a fixed full scale sinusoidal input at $f_{i}=98.3 \mathrm{KHz}$. 
As well, for completeness, the measured power consumption of the ADC core is presented in Fig. 6.28. At the desired frequency of operation of $25 \mathrm{MHz}$ the ADC core power dissipation is $100 \mu \mathrm{W}$. It was possible to measure the core power consumption since the comparator core is on separate power supply ring from the power hungry output buffers.

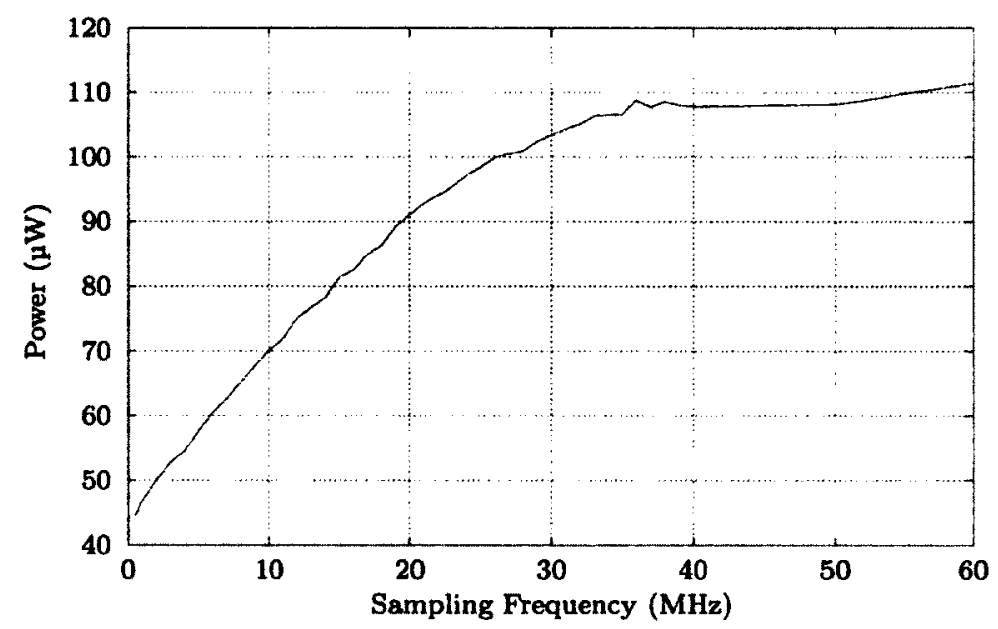

Figure 6.28: ADC core power consumption as a function of the sampling frequency $f_{s}$ for a fixed full scale sinusoidal input at $f_{i}=98.3 \mathrm{KHz}$. 


\subsection{Summary of Measurement Results}

A complete summary of the static and dynamic performance metrics extracted from measurement results of the ADC is presented in $\mathrm{Tb}$. 6.3. The ASIC was implemented in a IBM CMRF8SF $130 \mathrm{~nm}$ technology for a power supply of $1.2 \mathrm{~V}$. The input range was $600 \mathrm{mV}$ peak-to-peak differential. The ADC achieved an excellent power consumption for the core in terms of the FOM of $15 \mathrm{fJ}$ per conversion and core power consumption of $100 \mu \mathrm{W}$. The maximum sampling frequency while driving the external $\mathrm{SAH}$ at is full scale input is $4 \mathrm{MHz}$ which fundamentally limits the operation of the CABS ADC. Using a non-optimum calibration code the INL was improved to 11 LSB from $64 \mathrm{LSB}$ and the DNL was improved from $-68 \mathrm{LSB}$ to $14 \mathrm{LSB}$. The ENOB for an input frequency of $50 \mathrm{kHz}$ was 5.1 bits. In Fig. 6.29 and Fig. 6.30 the frequency and resolution of the fabricated ADC with a non-optimum calibration code are presented alongside current published ADCs with similar performance metrics.

Table 6.3: Summary of measurement results for non-optimum calibration code.

\begin{tabular}{cc}
\hline Metric & Measured Value \\
\hline Technology & IBM CMRF8SF 130 nm \\
VDD & $1.2 \mathrm{~V}$ \\
Input Range & $600 \mathrm{mV}_{\text {pk-pk,dif }}$ \\
Sample Rate & $4 \mathrm{MHz}$ \\
INL & $11 \mathrm{LSB}$ \\
DNL & $-14 \mathrm{LSB}$ \\
SINAD @ 50 kHz & $32.3 \mathrm{~dB}$ \\
SFDR @ 50 kHz & $35.7 \mathrm{~dB}$ \\
ENOB @ DC & 5.1 \\
Area & $4.064 \mathrm{~mm}^{2}$ \\
Power & $100 \mathrm{\mu W}$ \\
FOM & $15 \mathrm{fJ}$ \\
\hline
\end{tabular}




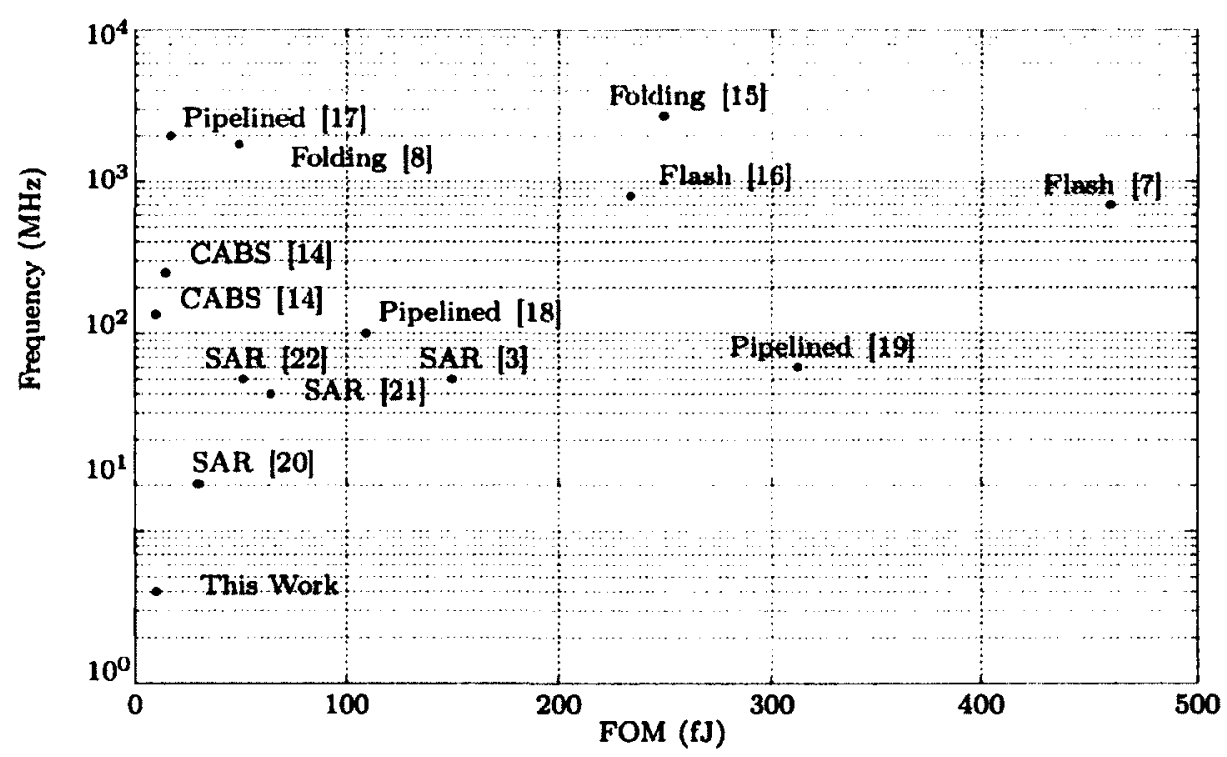

Figure 6.29: Comparison of frequency of operation a function of the figure of merit for published ADCs and measured CABS ADC performance.

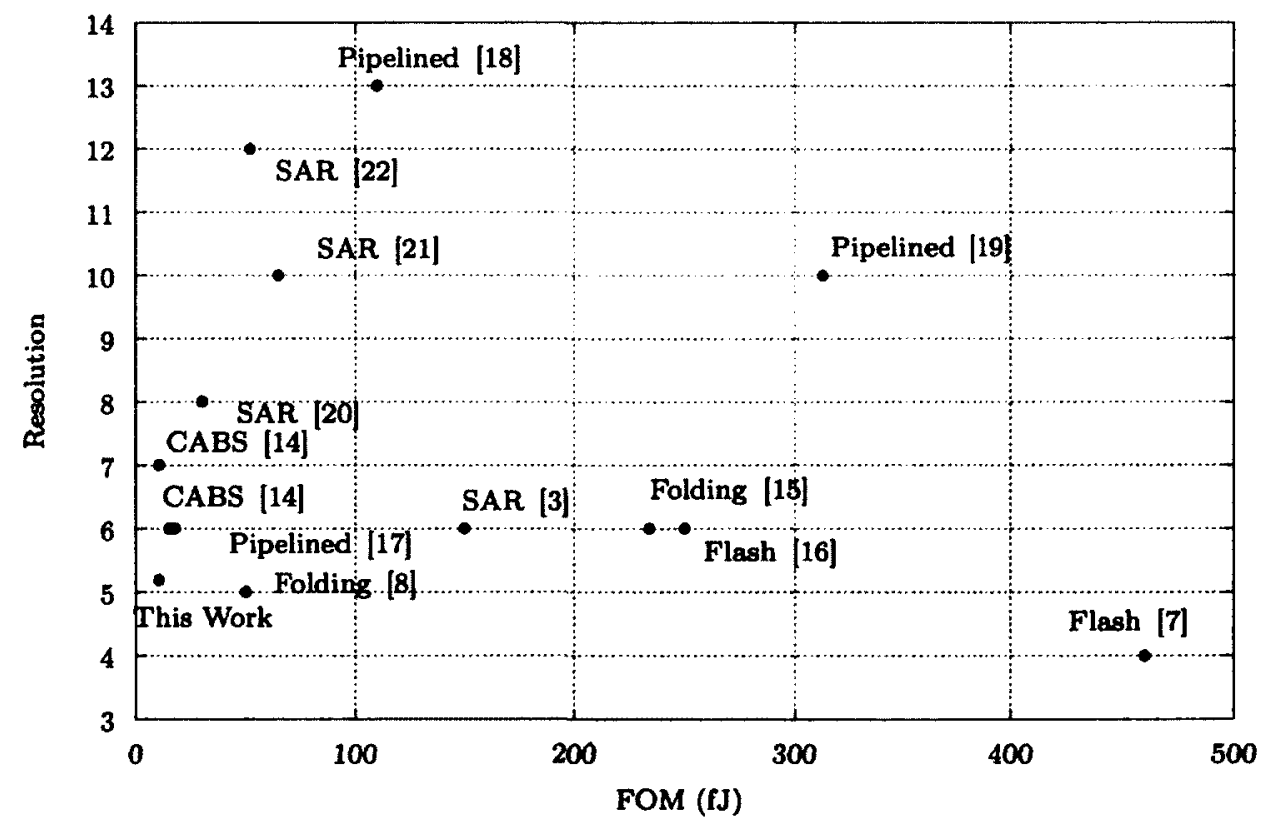

Figure 6.30: Comparison of $A D C$ resolution as a function of the figure of merit for published ADCs and measured CABS ADC performance. 
Table 6.4: Comparison of measurement results to published state-of-the art low power CMOS based ADC designs.

\begin{tabular}{|c|c|c|c|c|c|c|c|c|c|}
\hline Architecture & VDD & Technology & Area $\left(\mathrm{mm}^{2}\right)$ & Power (mW) & Speed (MS/s) & Input Range (mV,diff) & Resolution & ENOB & FOM \\
\hline This Work & $1.2 \mathrm{~V}$ & $0.13 \mu \mathrm{m}$ & 4 & 0.1 & 15 & 600 & 8 & 6.1 & 18 \\
\hline CABS [14] & $1 \mathrm{~V}$ & $90 \mathrm{~nm}$ & 0.050 & 0.140 & 250 & 384 & 6 & 5.3 & 15.0 \\
\hline CABS [14] & $1 \mathrm{~V}$ & $90 \mathrm{~nm}$ & 0.055 & 0.133 & 133 & 768 & 7 & 6.4 & 10.4 \\
\hline Folding [8] & $1 \mathrm{~V}$ & $90 \mathrm{~nm}$ & 0.017 & 2.2 & 1750 & 800 & 5 & 4.7 & 50.0 \\
\hline Folding [15] & $1 \mathrm{~V}$ & $90 \mathrm{~nm}$ & 0.360 & 50 & 2700 & 800 & 6 & NS & 250.0 \\
\hline Flash [7] & $1.8 \mathrm{~V}$ & $0.18 \mu \mathrm{m}$ & NS & 4.43 & 700 & NS & 4 & 3.8 & 460.0 \\
\hline Flash [16] & $1.2 \mathrm{~V}$ & $60 \mathrm{~nm}$ & 0.130 & 12 & 800 & NS & 6 & 5.6 & 234.0 \\
\hline Pipelined [17] & $1.1 \mathrm{~V}$ & $40 \mathrm{~nm}$ & 0.030 & 2.3 & 2000 & 640 & 6 & 5.5 & 17.4 \\
\hline Pipelined [18] & $1.3 \mathrm{~V}$ & $0.13 \mu \mathrm{m}$ & 1.200 & 91 & 100 & 2000 & 13 & NS & 110.0 \\
\hline Pipelined [19] & $1.2 \mathrm{~V}$ & $0.13 \mu \mathrm{m}$ & 0.980 & 19.2 & 60 & NS & 10 & NS & 313.0 \\
\hline SAR [3] & $1 \mathrm{~V}$ & $90 \mathrm{~nm}$ & 0.055 & 0.240 & 50 & 320 & 6 & 5.0 & 150.0 \\
\hline SAR [20] & $1 \mathrm{~V}$ & $90 \mathrm{~nm}$ & 0.132 & 0.069 & 10 & 400 & 8 & 7.8 & 30.0 \\
\hline SAR [21] & $1 \mathrm{~V}$ & $65 \mathrm{~nm}$ & 0.060 & 1.21 & 40 & 1000 & 10 & 8.9 & 65.0 \\
\hline SAR [22] & $1.3 \mathrm{~V}$ & $90 \mathrm{~nm}$ & 0.160 & 3.6 & 50 & 2000 & 12 & 10.6 & 52.0 \\
\hline
\end{tabular}




\section{Chapter 7}

\section{Conclusion}

\subsection{Contributions to Research}

In this work, the design of a hybrid 8-bit CABS based ADC was examined from preliminary system level design goals to in depth circuit design of low level components. To facilitate the design of the CABS ADC, a library of mixed signal analog cells was designed from the ground up. These cells included flip-flops, transmission gates, muxes as well as cells more specific to this architecture such as the dynamic latched comparator, SCMTA tuning arrays and bitline drivers. The performance of these mixed signal cells was analyzed using Monte Carlo simulations to gain insight into system level performance based on PVT variations of MOS devices. The state of the art resolution for a pure CABS architecture was extended from 6 bits to 8 bits through optimization and concurrent design of critical ADC building blocks. Simulation results placed the ADC designed in this work within the moderate to high resolution and excellent FOM range of ADCs. At the expense of an improvement in resolution the frequency of the ADC was lower compared to the majority of ADCs with similar

performance metrics. In terms of the FOM, compared to the critical benchmark of this design, the 8-bit CABS ADC achieved a conversion power of $15 \mathrm{fJ}$ for a resolution 
higher by two bits than the current state of the art CABS ADC [14]. The conversion power is measured at a desired operating frequency of $25 \mathrm{MHz}$. The core power consumption at $25 \mathrm{MHz}$ was $100 \mu \mathrm{W}$. Measurement results required the use of an external SAH circuit, which reduced the frequency of operation of the ADC. Using a non-optimum calibration code based on the nominal calibration code extracted from simulation measurement the INL of the fabricated ADC was improved from $64 \mathrm{LSB}$ to 11 LSB. Similarly the DNL was improved from -68 LSB to 14 LSB. Using the same calibration code the dynamic performance metrics of the $A / D$ converter were measured resulting in an ENOB of 5.1 bits improved from 3.1 bits. As well, the SINAD was $32.3 \mathrm{~dB}$ and the SFDR was $35.7 \mathrm{~dB}$ for a full scale input at an input frequency of $50 \mathrm{kHz}$ and a sampling frequency of $4 \mathrm{MHz}$.

\subsection{Future Work}

The fundamental limitation of the ADC presented in this work is the lack of an internal SAH. The SAH was not placed on the IC due to lack of space on the IC as well as time constraints. If not designed adequately, a critical front end block such as a SAH can degrade the performance of ADC by lowering SINAD and THD or not functioning altogether, removing the opportunity of testing the ADC in any capacity. A SAH design as described in [59] or [60] could be adapted for a $0.13 \mu \mathrm{m}$ technology node removing the frequency constraints imposed by external monolithic SAH ICs.

Leaving the SAH consideration behind, an aspect of the ADC which warrants the greatest amount of consideration is the improvement of the frequency of operation. To maintain the same resolution, the design could be migrated to a deeper sub micron technology node such as $65 \mathrm{~nm}$ or even $45 \mathrm{~nm}$. CMOS devices in progressive technology nodes have a higher transconductance and larger transit frequency making 
them ideal for high speed calibration based architectures. ${ }^{73}$ Since the layout area is proportional to $2^{\mathrm{N}}$ a smaller technology node would allow for a higher resolution in the same IC real estate area.

The CABS ADC could benefit from more sophisticated further hybridization with other ADC strategies such as pipelining or pre-amplification. Any existing pipelined or folding Flash structure could have its Flash converter replaced by a CABS ADC. Pipelining could conceivably improve the frequency of operation, as less overhead calibration would be required in each stage but the requirement of a DAC with a certain settling time would decrease from the improvement obtained through pipelining. Pre-amplification could be used to amplify the input through the smallest delay paths of the ADC as long the delay through those paths is the upper limit on the maximum operating frequency ${ }^{74}$. This would lower the duration the comparator spends in the metastable region of operation. Realistically, any frequency improvement would come at the expense of addition power consumption which, depending on the target application, might be undesirable altogether.

With the minimum MOSCAP device size being limited by physical design rules in the CMOS technology node, there is a fundamental limit for the minimum capacitance MOSCAP device. This in turn places a limit on the achievable resolution. As the resolution increases the tuning precision of the MOSCAP decreases eventually becoming greater than 1 LSB of the device. One potential scenario is to increase the input range of the ADC, increasing the dynamic range and the size of one LSB in the ADC. This is not a trivial matter. Because of IDWI of the devices, any increase requires larger and larger capacitances on regeneration nodes. This increases the conversion delay and cripples the frequency of operation. By moving towards a

\footnotetext{
${ }^{73}$ The requirement of calibration is even stronger with smaller technology nodes as the effects of PVT increase.

${ }^{74}$ This only applies as long as the largest capacitance paths with the highest IDWIs have a lower propagation delay than their low voltage input delay paths.
} 
newer technology node, comparators with the same devices could benefit from the reduced capacitive footprint of the minimum sized FET which could be fabricated in the process. A more interesting approach would be to use programable floating gate references such as those presented in $[61,62]$. In [62] a 13 bit programable voltage reference was achievable using floating gate techniques.

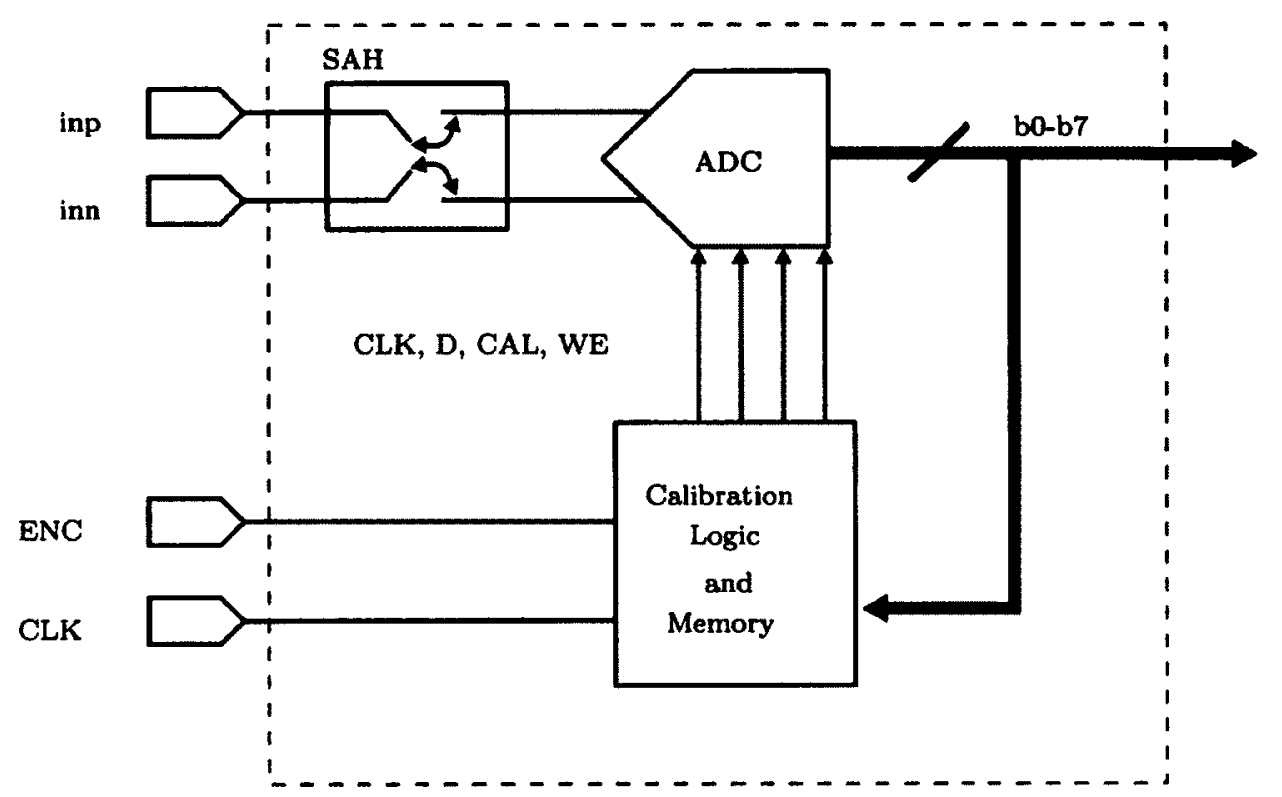

Figure 7.1: Functional diagram of SoC calibration of the CABS ADC.

Finally the lack of an on chip calibration strategy is a significant drawback for any possible implementation in a receiver chain or SoC solution. It would therefore be of great interest to implement the tuning algorithm for the CABS ADC using register transfer level (RTL) logic design, preferably on the same IC as is shown in Fig. 7.1. The calibration strategy ${ }^{75}$ is based on the binary search strategy presented in [14]. The "ENC" input enables calibration of the ADC. If the input of the CABS ADC corresponds to the desired threshold for the comparator, the difference between the desired threshold or measured threshold should approach zero. Each tuning code is

\footnotetext{
${ }^{75}$ This refers to the calibration strategy proposed in [14].
} 
$5.28 \mathrm{k}$ Bits. Depending on the calibration strategy adopted, the calibration controller would have to access a significant memory on the order of megabytes. In addition this example assumes the differential input could be generated by a DAC in the same package or on the same PCB. This would be convenient if a DAC of that resolution is required in an $\mathrm{SoC}$ solution. In the case of any device which transmits and receives data, such as a wireless networking card or sensor node, both ADC and DAC components are present. The calibration of the ADC would then fall on DSP block already present in the system. 


\section{List of References}

[1] W. Kester, The Data Conversion Handbook. New York: Elsevier, 2005.

[2] F. Maloberti, Data converters. Berlin: Springer, 2007.

[3] P. Nuzzo, C. Nani, C. Armiento, A. Sangiovanni-Vincentelli, J. Craninckx, and G. Van der Plas, "A 6-Bit 50-MS/s Threshold Configuring SAR ADC in 90-nm Digital CMOS," Circuits and Systems I: Regular Papers, IEEE Transactions on, vol. 59, pp. $80-92$, Jan. 2012.

[4] A. Annema, B. Nauta, R. van Langevelde, and H. Tuinhout, "Analog circuits in ultra-deep-submicron CMOS," Solid-State Circuits, IEEE Journal of, vol. 40, pp. $132-143$, Jan. 2005.

[5] Y. Chiu, B. Nikolic, and P. Gray, "Scaling of analog-to-digital converters into ultra-deep-submicron CMOS," in Custom Integrated Circuits Conference, 2005. Proceedings of the IEEE 2005, pp. 375 -382, Sept. 2005.

[6] B. Murmann, P. Nikaeen, D. Connelly, and R. Dutton, "Impact of Scaling on Analog Performance and Associated Modeling Needs," Electron Devices, IEEE Transactions on, vol. 53, pp. $2160-2167$, Sept. 2006.

[7] G. Torfs, Z. Li, J. Bauwelinck, X. Yin, G. Van der Plas, and J. Vandewege, "Low-power 4-bit flash analogue to digital converter for ranging applications," Electronics Letters, vol. 47, pp. 20 -22, June 2011.

[8] B. Verbruggen, J. Craninckx, M. Kuijk, P. Wambacq, and G. Van der Plas, "A $2.2 \mathrm{~mW} 1.75 \mathrm{GS} / \mathrm{s} 5$ Bit Folding Flash ADC in $90 \mathrm{~nm}$ Digital CMOS," Solid-State Circuits, IEEE Journal of, vol. 44, pp. 874 -882, March 2009.

[9] B. Verbruggen, P. Wambacq, M. Kuijk, and G. Van der Plas, "A 7.6 mW 1.75 GS/s 5 bit flash A/D converter in $90 \mathrm{~nm}$ digital CMOS," in VLSI Circuits, 2008 IEEE Symposium on, pp. $14-15$, June 2008. 
[10] R. Taft, P. Francese, M. Tursi, O. Hidri, A. MacKenzie, T. Hohn, P. Schmitz, H. Werker, and A. Glenny, "A $1.8 \mathrm{~V} 1.0 \mathrm{GS} / \mathrm{s}$ 10b Self-Calibrating UnifiedFolding-Interpolating ADC With 9.1 ENOB at Nyquist Frequency," Solid-State Circuits, IEEE Journal of, vol. 44, pp. 3294 -3304, Dec. 2009.

[11] B. Kim, D. Kim, J. Hwang, J. Moon, and M. Song, "12-bit 80MSPS double folding/interpolation A/D converter," in SoC Design Conference, 2008. ISOCC '08. International, vol. 03, pp. III-1 -III-2, Nov. 2008.

[12] T. Louwsma, S. and B. Nauta, Time-interleaved analog-to-digital converters. Dordrecht: Springer, 2011.

[13] G. Manganaro, Advanced Data Converters. Cambridge: Cambridge University Presss, 2012.

[14] G. Van der Plas and B. Verbruggen, "A $150 \mathrm{MS} / \mathrm{s} 133 \mu \mathrm{W} 7$ bit ADC in $90 \mathrm{~nm}$ Digital CMOS," Solid-State Circuits, IEEE Journal of, vol. 43, pp. $2631-2640$, Dec. 2008.

[15] Y. Nakajima, A. Sakaguchi, T. Ohkido, N. Kato, T. Matsumoto, and M. Yotsuyanagi, "A Background Self-Calibrated $6 \mathrm{~b} 2.7 \mathrm{GS} / \mathrm{s}$ ADC With CascadeCalibrated Folding-Interpolating Architecture," Solid-State Circuits, IEEE Journal of, vol. 45, pp. $707-718$, April 2010.

[16] C.-Y. Chen, M. Le, and K. Y. Kim, "A Low Power 6-bit Flash ADC With Reference Voltage and Common-Mode Calibration," Solid-State Circuits, IEEE Journal of, vol. 44, pp. 1041 -1046, April 2009.

[17] B. Verbruggen, J. Craninckx, M. Kuijk, P. Wambacq, and G. Van der Plas, "A $2.6 \mathrm{~mW} 6$ bit 2.2 GS/s Fully Dynamic Pipeline ADC in $40 \mathrm{~nm}$ Digital CMOS," Solid-State Circuits, IEEE Journal of, vol. 45, pp. 2080 -2090, Oct. 2010.

[18] D.-H. Hwang, J.-E. Song, S.-P. Nam, H.-J. Kim, T.-J. An, K.-S. Kim, and S.H. Lee, "A range-scaled 13b 100MS/s $130 \mathrm{~nm}$ CMOS SHA-free ADC based on a single reference," in SoC Design Conference (ISOCC), 2011 International, pp. 62 -65 , Nov. 2011.

[19] H.-C. Choi, Y.-J. Kim, S.-W. Yoo, S.-Y. Hwang, and S.-H. Lee, "A Programmable 0.8-V 10-bit 60-MS/s 19.2-mW 0.13um CMOS ADC Operating Down to 0.5 V", Circuits and Systems II: Express Briefs, IEEE Transactions on, vol. 55, pp. 319 -323, April 2008. 
[20] P. Harpe, C. Zhou, X. Wang, G. Dolmans, and H. de Groot, "A 30fJ/conversionstep 8b 0-to-10MS/s asynchronous SAR ADC in 90nm CMOS," in Solid-State Circuits Conference Digest of Technical Papers (ISSCC), 2010 IEEE International, pp. $388-389$, Feb. 2010.

[21] M. Furuta, M. Nozawa, and T. Itakura, "A 10-bit, 40-MS/s, $1.21 \mathrm{~mW}$ Pipelined SAR ADC Using Single-Ended 1.5-bit/cycle Conversion Technique," Solid-State Circuits, IEEE Journal of, vol. 46, pp. 1360 -1370, June 2011.

[22] C. Lee and M. Flynn, "A SAR-Assisted Two-Stage Pipeline ADC," Solid-State Circuits, IEEE Journal of, vol. 46, pp. 859 -869, April 2011.

[23] P. Nuzzo, C. Nani, C. Armiento, A. Sangiovanni-Vincentelli, J. Craninckx, and G. Van der Plas, "A 6-Bit 50-MS/s Threshold Configuring SAR ADC in 90-nm Digital CMOS," Circuits and Systems I: Regular Papers, IEEE Transactions on, vol. PP, no. 99, p. 1, 2011.

[24] T. Kobayashi, K. Nogami, T. Shirotori, and Y. Fujimoto, "A current-controlled latch sense amplifier and a static power-saving input buffer for low-power architecture," Solid-State Circuits, IEEE Journal of, vol. 28, pp. 523 -527, April 1993.

[25] B. Wicht, T. Nirschl, and D. Schmitt-Landsiedel, "A yield-optimized latch-type SRAM sense amplifier," in Solid-State Circuits Conference, 2003. ESSCIRC '03. Proceedings of the 29th European, pp. 409 - 412, Sept. 2003.

[26] P. Nuzzo, G. Van der Plas, R. De Bernardinis, L. Van der Perre, B. Gyselinckx, and P. Terreni, "A $10.6 \mathrm{~mW} / 0.8 \mathrm{pJ}$ power-scalable $1 \mathrm{GS} / \mathrm{s} 4 \mathrm{~b}$ ADC in $0.18 \mu \mathrm{m}$ CMOS with 5.8GHz ERBW," in Design Automation Conference, 2006 43rd $A C M / I E E E$, pp. $873-878$, Jan. 2006.

[27] A. Nikoozadeh and B. Murmann, "An Analysis of Latch Comparator Offset Due to Load Capacitor Mismatch," Circuits and Systems II: Express Briefs, IEEE Transactions on, vol. 53, pp. $1398-1402$, Dec. 2006.

[28] P. Kinget, "Device mismatch and tradeoffs in the design of analog circuits," Solid-State Circuits, IEEE Journal of, vol. 40, pp. 1212 - 1224, June 2005.

[29] J. Croon, M. Rosmeulen, S. Decoutere, W. Sansen, and H. Maes, "An easy-to-use mismatch model for the MOS transistor," Solid-State Circuits, IEEE Journal of, vol. 37, pp. 1056 - 1064, Aug 2002. 
[30] M. Pelgrom, H. Tuinhout, and M. Vertregt, "Transistor matching in analog CMOS applications," in Electron Devices Meeting, 1998. IEDM '98 Technical Digest., International, pp. 915 -918, Dec 1998.

[31] P. Drennan and C. McAndrew, "A comprehensive MOSFET mismatch model," in Electron Devices Meeting, 1999. IEDM Technical Digest. International, pp. 167 $-170,1999$.

[32] P. Drennan and C. McAndrew, "Understanding MOSFET mismatch for analog design," Solid-State Circuits, IEEE Journal of, vol. 38, pp. 450 - 456, Mar 2003.

[33] P. M. Figueiredo and J. C. Vital, Offset reduction techniques in high-speed analogto-digital converters. Dordrecht: Springer, 2009.

[34] J. D and M. K, Analog Integrated Circuit Design. New York: John Wiley and Sons, 2000.

[35] "CMOS8RF (CMRF8SF) Design Manual," tech. rep., IBM Microelectronics Division, 2010.

[36] Y. Shi, T. Ma, S. Prasad, and S. Dhanda, "Polarity dependent gate tunneling currents in dual-gate CMOSFETs ," Electron Devices, IEEE Transactions on, vol. 45, pp. $2355-2360$, Nov 1998.

[37] A. B. Bhattacharyya, Compact MOSFET models for VLSI design. Singapore: John Wiley and Sons (Asia), 2009.

[38] N. H. E. Weste and D. M. Harris, CMOS VLSI design: a circuits and systems perspective. Boston: Addison-Wesley, 2010.

[39] S.-M. Kang and Y. Leblebici., CMOS Digital Integrated Circuits: Analysis and Design. Boston: McGraw-Hill, 2003.

[40] B. N. J. M. Rabaey, A. P. Chandrakasan, Digital integrated circuits: a design perspective. New York: Pearson Education, 2003.

[41] B. Cherkauer and E. Friedman, "Unification of speed, power, area, and reliability in CMOS tapered buffer design," in Circuits and Systems, 1994. ISCAS '94., 1994 IEEE International Symposium on, vol. 4, pp. 111 -114 vol.4, May 1994.

[42] H. W. Johnson and M. Graham, High-speed signal propagation: Advanced black magic. Upper Saddle River, NJ: Prentice Hall PTR, 2002. 
[43] C. Saint and J. Saint, IC mask design: Essential layout techniques; (practical layout reference for circuit designers and mask designers; techniques for matching, noise, high frequency issues and more; step by step case studies detailing CMOS and bipolar RFIC/. New York: McGraw-Hill, 2002.

[44] D. Clein, CMOS IC layout : concepts, methodologies, and tools. Boston: Newnes, 2000.

[45] S. W. Smith, Digital signal processing: A practical guide for engineers and scientists. Amsterdam: Newnes, 2003.

[46] D. Dallet and J. M. Silva, Dynamic characterisation of analogue-to-digital converters. Dordrecht: Springer, 2005.

[47] N. Gray, $A B C s$ of $A D C s$. National Semiconductor, 2006.

[48] "Dynamic Testing of High-Speed ADCs," tech. rep., Maxim Integrated Products, 2001. http://pdfserv.maxim-ic.com/en/an/AN729. pdf; accessed March 30, 2012.

[49] K. Mitzner, Complete PCB design using OrCad capture and layout. Amsterdam: Newnes, 2007.

[50] B. Archambeault, PCB design for real-world EMI control. Boston: Kluwer Academic, 2002.

[51] "ADG3308 Data Sheet," tech. rep., MAXIM, February 2012. http:// datasheets.maxim-ic.com/en/ds/DS1843.pdf; accessed March 30, 2012.

[52] "AD8139 Data Sheet," tech. rep., Analog Devices, Octobre 2007. http: //www.analog.com/static/imported-files/data_sheets/AD8139.pdf; accessed March 30, 2012.

[53] "AD783 Data Sheet," tech. rep., Analog Devices, Octobre 1992. http:// www analog.com/static/imported-files/data_sheets/AD783.pdf; accessed March 30, 2012.

[54] "AD4853 Data Sheet," tech. rep., Analog Devices, January 2011. http://www . analog. com/static/imported-files/data_sheets/ADA4853-1_ 4853-2_4853-3.pdf; accessed March 30, 2012. 
[55] "AD8475 Data Sheet," tech. rep., Analog Devices, April 2011. http: //www.analog.com/static/imported-files/data_sheets/AD8475.pdf; accessed March 30, 2012.

[56] "ADG3308 Data Sheet," tech. rep., Analog Devices, Septembre 2007. http://www analog.com/static/imported-files/data_sheets/ADG3308_ 3308-1.pdf; accessed March 30, 2012.

[57] "CY74FCT2574T Data Sheet," tech. rep., Texas Instruments, Octobre 2011.

[58] "ADG3308 Data Sheet," tech. rep., Analog Devices, Septembre 2005. http: //www. analog. com/static/imported-files/data_sheets/AD9513.pdf; accessed March 30, 2012.

[59] H. Wang, H. Hong, L. Sun, and Z. Yu, "A sample-and-hold circuit for 10-bit 100MS/s pipelined ADC," in ASIC (ASICON), 2011 IEEE 9th International Conference on, pp. $480-483$, Oct. 2011.

[60] D. Vecchi, C. Azzolini, A. Boni, F. Chaahoub, and L. Crespi, "100-MS/s 14-b track-and-hold amplifier in 0.18um CMOS," in Solid-State Circuits Conference, 2005. ESSCIRC 2005. Proceedings of the 31st European, pp. 259 - 262, Sept. 2005.

[61] P. Brady and P. Hasler, "Offset compensation in flash ADCs using floatinggate circuits," in Circuits and Systems, 2005. ISCAS 2005. IEEE International Symposium on, pp. $6154-6157$ Vol. 6, May 2005.

[62] C. Huang, P. Sarkar, and S. Chakrabartty, "Rail-to-Rail, Linear Hot-Electron Injection Programming of Floating-Gate Voltage Bias Generators at 13-Bit Resolution," Solid-State Circuits, IEEE Journal of, vol. 46, pp. 2685 -2692, Nov. 2011. 


\section{Appendix A}

\section{Bill of Materials for PCB1}

Table A.1: Bill of materials for the preliminary PCB1.

\begin{tabular}{cl}
\hline Quantity & Manufacturer \\
\hline 6 & Nichicon \\
6 & Kemet \\
6 & Murata \\
6 & TDK \\
6 & Molex Connector Corporation \\
3 & Vishay Thin Film \\
3 & Emerson \\
6 & TE Connectivity \\
1 & 3 M \\
4 & Littelfuse Inc
\end{tabular}

Manufacturer ID
TCM0J106M8R
C0603C104K8RACTU
GQM2195C1H270JB01D
MMZ1608Y102B
71430-0268
FC0603E50R0BTBST1
142-0701-201
A30771-ND
961240-6300-AR-PR
SP3010-04UTG
Description

CAP TANT 10UF 6.3V $20 \% 0603$ CAP CER O.1UF 10V $10 \%$ X7R 0603 CAP CER 27PF 5OV 5\% NPO 0603 FERRITE CHIP 1000 OHM 400MA 060 CONN RECEPT R/A .8MM G8POS PCB RES 50 OHM $125 \mathrm{MW} .1 \% 0603$ SMD CONN RECEPT STRAIGHT PCB .155" G CONN HEADER VERT 3POS .100 30AU CONN HEADER VERT 3POS , 100 3OAU TVS ARRAY ESD 4CH .45PF 8KV UDFN 


\section{Appendix B}

\section{Bill of Materials for PCB2}

Table B.1: Bill of materials for PCB2.

\begin{tabular}{|c|c|c|c|}
\hline Quantity & Manufacturer & Manufacturer ID & Description \\
\hline 1 & Analog Devices & ADA 4853-1,2,3 & IC OPAMP VF R-R 100MHZ SC70-6 \\
\hline 2 & Analog Devices & AD8139ACPZ-REEL7CT-ND & IC AMP DIFF R-R LN LDIST BSOIC \\
\hline 2 & Analog Devices & AD8475ARMZ & IC OP AMP R-R FUNNEL 10 MSOP \\
\hline 1 & Intersil & ISL21070DIH306Z-TKTR-ND & IC VREF SERIES $0.6 \mathrm{~V}$ SOT-23-3 \\
\hline 1 & Analog Dovices & AD803LANZ & IC OPAMP VF R-R LP LDIST 8DIP \\
\hline 1 & Fairchild Semiconductor & FAN2558S12X & IC REG LDO 180MA $1.2 \mathrm{~V}$ SOT23-5 \\
\hline 1 & Fairchild Serniconductor & FAN2500S33X & IC REG LDO CMOS 3.3V SOT-23-5 \\
\hline 1 & Linear Technology & LT3032EDE & IC REG LDO +/-5V .15A DL 14DFN \\
\hline 1 & Analog Devicos & AD783JRZ & IC AMP SAMPLE HOLD LP 5MA BSOIC \\
\hline 1 & Maxim Integrated Products & DS1843D+ & IC CIRCUIT SAMPLE-N-HOLD \&-UDFN \\
\hline 1 & Anslog Devices & AD9513BCPZ & IC CLOCK DIST 3OUT PLL 32LFCSP \\
\hline 1 & Analog Devicea & ADG3301BKSZ-REEL7 & TRANSLATOR SGL LL BIDIR SC70-6 \\
\hline 1 & Analog Devices & ADG3308BRUZ & IC XLATOR BCH 1.2-5.5V 20-TSSOP \\
\hline 2 & Fairchild Semiconductor & NC7SZ58 & IC LOGIC GATE UNIV 2INPUT SC70-6 \\
\hline 1 & Texas Instrument. & CY74FCT2574ATQCT & IC D-TYPE F-F TRI-ST HS 20-QSOP \\
\hline 1 & Littelfuse Inc & SP3003-04JTG & TVS ARRAY ESD 4CH 0.65PF SC70-6 \\
\hline 1 & Littelfuse Inc & SP3003-08ATG & TVS DIODE ARRAY 8CH 0.65PF MSOP- \\
\hline 1 & FTDI & V2DIP1-32 & MOD VINCULUM-1I DEV 1 PORT 32DIP \\
\hline 1 & FTDI & VNC2 DEBUG MODULE & MOD VINCULUM-II DEBUGGR/PROGRAMR \\
\hline 1 & TE Connectivity & FSM4JSMATR & SWITCH TACTILE SPST-NO O.OBA $12 \mathrm{~V}$ \\
\hline 1 & Susumu & RG1608P-4121-B-TS & RES 4.12K OHM $1 / 10 \mathrm{~W} .1 \% 0603$ SMD \\
\hline 6 & Nichicon & TCMOJ 106M8R & CAP TANT 10UF 6.3V $20 \% 0603$ \\
\hline 20 & Kemet & C0803C104K8RACTU & CAP CER O.1UF 10V $10 \%$ X7R 0603 \\
\hline 6 & Murats & GQM2195C1H270JBO1D & CAP CER 27PF 50V 5\% NPO 0603 \\
\hline 1 & Murats & PV36W202C01B00 & TRIMMER 2K OHM O.5W TH \\
\hline 8 & Panasonic & RGH1608-2C-P-201-B & RES 200 OHM $1 / 6$ W $0.1 \% 0603$ SMD \\
\hline 1 & Yangeo & RC0603FR-074K02L & RES $4.02 \mathrm{~K}$ OHM $1 / 10 \mathrm{~W} 1 \% 0603$ SMD \\
\hline 1 & Yangeo & RC0603FR-078K06L & RES 8.06K OHM 1/10W $1 \% 0603$ SMD \\
\hline $\mathbf{1}$ & Yangeo & RC0603JR-0716KL & RES 16K OHM $1 / 10$ W $5 \% 0603$ SMD \\
\hline 1 & Yangeo & RC0603FR-071K02L & RES $1.02 \mathrm{~K}$ OHM $1 / 10 \mathrm{~W} 1 \% 0603$ SMD \\
\hline 6 & Yangeo & RC0603FR-0715RL & RES 15.0 OHM 1/10W $1 \% 0603$ SMD \\
\hline 6 & Murata & GRM188BC1H271JAOID & CAP CER 270PF 50V 5\% NPO 0603 \\
\hline 8 & Murata & GRM1885C1H132JAO1D & CAP CER 1300PF 50V 5\% NPO 0603 \\
\hline
\end{tabular}

